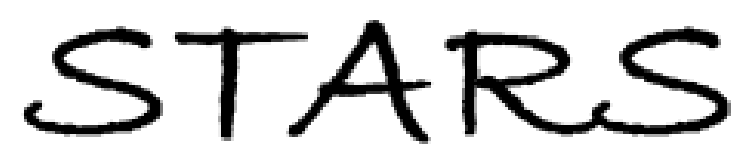

University of Central Florida

STARS

Electronic Theses and Dissertations, 2004-2019

2005

\title{
Tunable Liquid Crystal Photonic Devices
}

Yun-Hsing Fan

University of Central Florida

Part of the Electromagnetics and Photonics Commons, and the Optics Commons

Find similar works at: https://stars.library.ucf.edu/etd

University of Central Florida Libraries http://library.ucf.edu

This Doctoral Dissertation (Open Access) is brought to you for free and open access by STARS. It has been accepted for inclusion in Electronic Theses and Dissertations, 2004-2019 by an authorized administrator of STARS. For more information, please contact STARS@ucf.edu.

\section{STARS Citation}

Fan, Yun-Hsing, "Tunable Liquid Crystal Photonic Devices" (2005). Electronic Theses and Dissertations, 2004-2019. 314.

https://stars.library.ucf.edu/etd/314

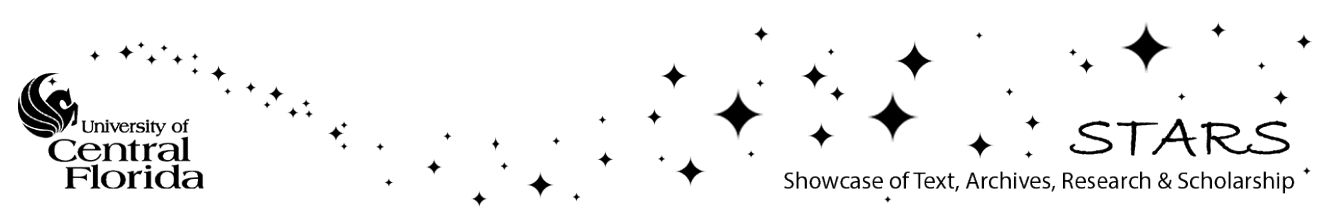




\title{
TUNABLE LIQUID CRYSTAL PHOTONIC DEVICES
}

\author{
by \\ YUN-HSING FAN \\ BS in Physics, Fu-Jen Catholic University, 1997 \\ MS in Physics, National Cheng Kung University, 1999 \\ A dissertation submitted in partial fulfillment of the requirements \\ for the degree of Doctor of Philosophy \\ in the College of Optics and Photonics \\ at the University of Central Florida \\ Orlando, Florida
}

Spring Term

2005

Major Professor: Shin-Tson Wu 
(C) 2005 Yun-Hsing Fan 


\begin{abstract}
Liquid crystal (LC)-based adaptive optics are important for information processing, optical interconnections, photonics, integrated optics, and optical communications due to their tunable optical properties. In this dissertation, we describe novel liquid crystal photonic devices and their fabrication methods. The devices presented include inhomogeneous polymer-dispersed liquid crystal (PDLC), polymer network liquid crystals (PNLC) and phase-separated composite film (PSCOF).

Liquid crystal/polymer composites could exist in different forms depending on the fabrication conditions. In Chap. 3, we demonstrate a novel nanoscale PDLC device that has inhomogeneous droplet size distribution. In such a PDLC, the inhomogeneous droplet size distribution is obtained by exposing the LC/monomer with a non-uniform ultraviolet (UV) light. An electrically tunable-efficiency Fresnel lens is devised for the first time using nanoscale PDLC. The tunable Fresnel lens is very desirable to eliminate the need of external spatial light modulator. Different gradient profiles are obtained by using different photomasks. The nanoscale LC droplets are randomly distributed within the polymer matrix, so that the devices are polarization independent and exhibit a fast response time. Because of the small droplet sizes, the operating voltage is higher than 100 $\mathrm{V}_{\text {rms }}$.
\end{abstract}

To lower the driving voltage, in Chap. 2 and Chap. 3, we have investigated a polymer-network liquid crystal (PNLC) using a rod-like monomer structure. Since the monomer concentration is only about $5 \%$, the operating voltage is below $10 \mathrm{~V}_{\text {rms. }}$ The 
PNLC devices are polarization dependent. To overcome this shortcoming, stacking two cells with orthogonal alignment directions is a possibility.

In Chap. 3, another approach to lower the operating voltage is to use phaseseparated composite film (PSCOF) where the LC and polymer are separated completely to form two layers. Without multi-domain formed in the LC cell, PSCOF is free from light scattering. Using PNLC and PSCOF, we also demonstrated LC blazed grating and Fresnel lens. The diffraction efficiency of these devices is continuously controlled by the electric field.

Besides Fresnel lens, another critical need for imaging and display is to develop a system with continuously tunable focal length. A conventional zooming system controls the lens distance by mechanical motion along the optical axis. This mechanical zooming system is bulky and power hungry. To overcome the bulkiness, in Chap. 4 we developed an electrically tunable-focus flat LC spherical lens which consists of a spherical electrode imbedded in the top flat substrates while a planar electrode on the bottom substrate. The electric field from the spherical and planar electrodes induces a centrosymmetric gradient refractive index distribution within the LC layer which, in turn, causes the focusing effect. The focal length is tunable by the applied voltage. A tunable range from $0.6 \mathrm{~m}$ to infinity is achieved.

Microlens array is an attractive device for optical communications and projection displays. In Chap. 5, we describe a LC microlens array whose focal length can be switched from positive to negative or vise versa by the applied voltage. The top spherical electrode glass substrate is flattened with a polymer layer. The top convex substrate and LC layer work together like a zoom lens. By tuning the refractive index profile of the LC 
layer, the focal length of the microlens array can be switched from positive to negative or vise versa. The tunable LC microlens array would be a great replacement of a conventional microlens array which can be moved by mechanical elements. The fast response time feature of our LC microlens array will be very helpful in developing 3-D animated images.

A special feature for LC/polymer composites is light scattering. The concept is analogous to the light scattering of clouds which consist of water droplets. In Chap. 6, we demonstrate polymer network liquid crystals for switchable polarizers and optical shutters. The PNLC can present anisotropic or isotropic light scattering behavior depending on the fabrication methods. The use of dual-frequency liquid crystal and special driving scheme leads to a sub-millisecond response time. The applications for display, light shutters, and switchable windows are emphasized.

Although polymer networks help to reduce liquid crystal response time, they tend to scatter light. In Chap. 7, for the first time, we demonstrate a fast-response and scattering-free homogeneously-aligned PNLC light modulator. Light scattering in the near-infrared region is suppressed by optimizing the polymer concentration such that the network domain sizes are smaller than the wavelength. As a result, the PNLC response time is $\sim 300 \mathrm{X}$ faster than that of a pure LC mixture except that the threshold voltage is increased by $\sim 25 \mathrm{X}$. The PNLC cell also holds promise for mid and long infrared applications where response time is a critical issue. 
To my Parents, Pin-I Fan and Befeng Shay

To my Husband, Zhen Ling 


\section{ACKNOWLEDGMENTS}

I would like to express my gratefulness to my advisor, Professor Shin-Tson $\mathrm{Wu}$, for his patient guidance, encouragement, and support throughout my studies at the College of Optics and Photonics/CREOL/FPCE in the University of Central Florida. I greatly appreciate his technical insights and providing the laboratory facilities in the development process of this dissertation. For me and for every student in the group, he set up a model of dedication to work. I sincerely believe that his advices and directions will benefit me in my future life both professionally and personally. Last year, I won the prestigious scholarship in Optical Science \& Technology from the International Society for Optical Engineering (SPIE) and the Outstanding Graduate Student Award of the Institute of Electrical and Electronics Engineers (IEEE) Orlando Section. I highly appreciate his effective mentoring, as I know that without his support, I would not be able to make such impressive achievements, progress and success in my research work.

I would also like to take this chance to express my thankfulness to my Ph.D. committee members, Dr. Glenn D. Boreman, Dr. Nabeel A. Riza, and Dr. Thomas X. Wu for their evaluating this dissertation and my doctoral qualifications.

Appreciations also go to all of my colleagues and friends, especially to Dr. Hongwen Ren, Yi-Hsin Lin, and Sakoolkan Boonruang. I want to thank them for their help on laboratory experiments and constant supports. The great friendship and entertainment from all these people was an important ingredient in my daily work life. 
During my graduate studies at UCF, the Defense Advanced Research Projects Agency (DARPA) and the Air Force Office of Scientific Research (AFOSR) have been continuously providing funding resources. I sincerely thank these two organizations for their financial aid.

Finally and foremost, I want to express my sincere gratitude to my parents, Pin-I Fan and Befeng Shay, for their priceless love, understanding, prayers, and blessings; while most importantly, I want to thank my husband, Zhen Ling, for his love, patience, and support on my research work to make all my achievements possible. 


\section{TABLE OF CONTENTS}

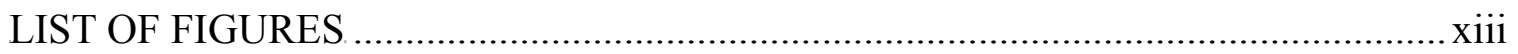

LIST OF TABLES

LIST OF ABBREVIATIONS...........................................................................................

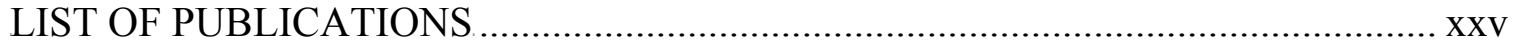

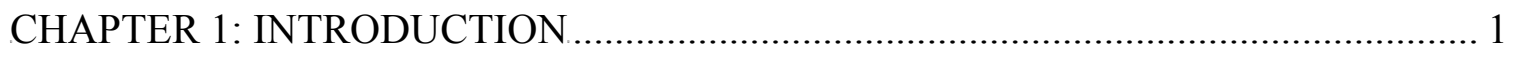

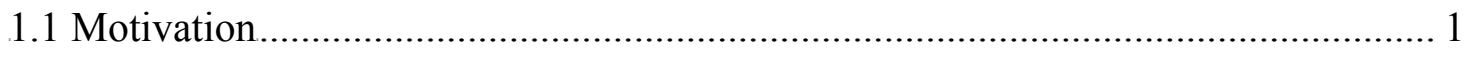

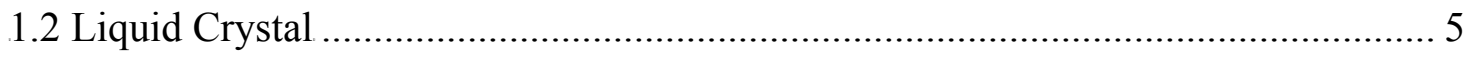

1.3 Nematic Liquid Crystals ................................................................................... 10

1.3 Inhomogeneous Nanoscale Polymer-Dispersed Liquid Crystal (PDLC) ................ 14

1.4 Polymer Network Liquid Crystal (PNLC) ............................................................ 18

1.5 Phase-Separated Composite Film (PSCOF) ………......................................... 22

CHAPTER 2: PRISM GRATING USING POLYMER STABILIZED NEMATIC

LIQUID CRYSTAL

2.1 Introduction: Beam Steering and Blazed Grating................................................. 27

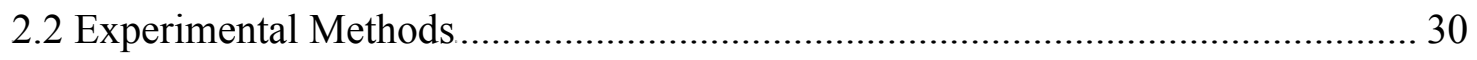

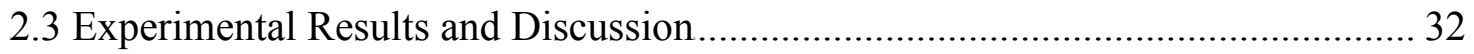

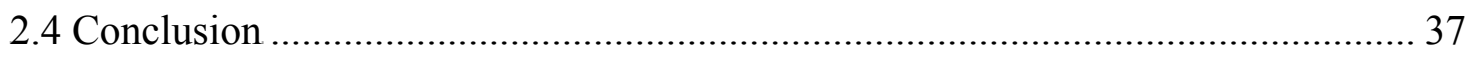

CHAPTER 3: SWITCHABLE FRESNEL LENS USING LIQUID CRYSTAL /

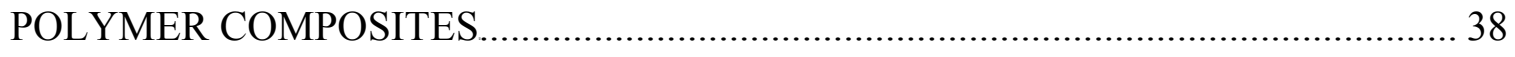

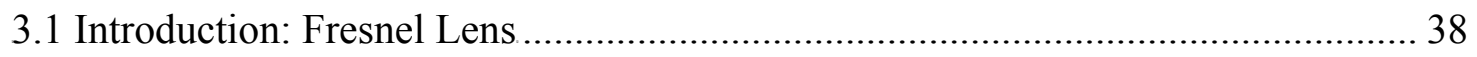


3.2 Theory of Binary Phase Fresnel Lenses................................................................ 40

3.3 Fresnel Lens Using Nanoscale Polymer-Dispersed Liquid Crystal (PDLC).......... 49

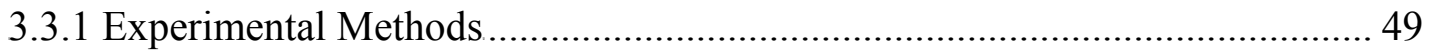

3.3.2 Operation Principle

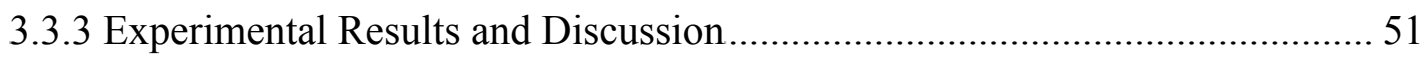

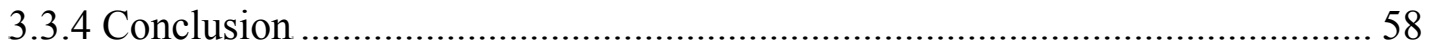

3.4 Fresnel Lens Using Polymer Network Liquid Crystals (PNLC) ............................ 58

3.4.1 Experimental Methods …………………………...................................... 58

3.4.2 Operation Principle

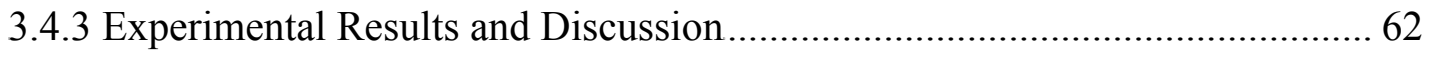

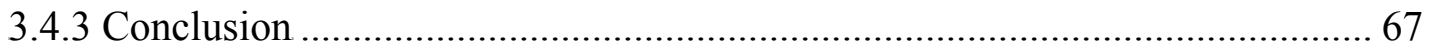

3.5 Fresnel Lens Using Phase-Separated Composite Film (PSCOF) ............................ 68

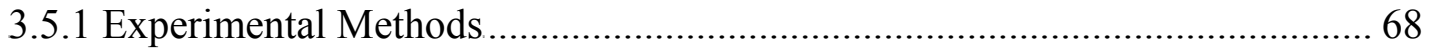

3.5.2 Operation Principle

3.5.3 Experimental Results and Discussion........................................................ 74

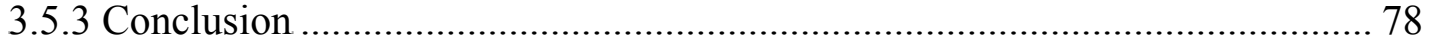

CHAPTER 4: TUNABLE-FOCUS FLAT LIQUID CRYSTAL SPHERICAL LENS ... 79

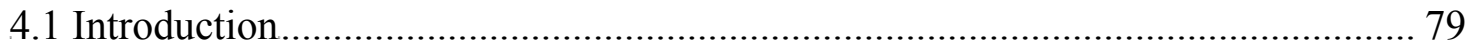

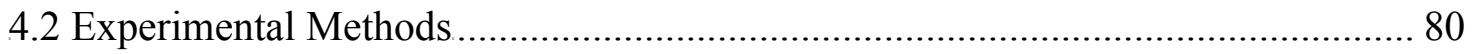

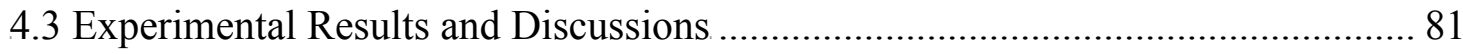

4.4 Numerical Calculation and Focal Length Prediction.............................................. 87

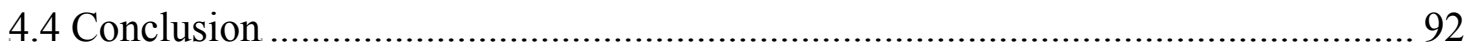


CHAPTER 5: LIQUID CRYSTAL MICROLENS ARRAYS WITH SWITCHABLE POSITIVE AND NEGATIVE FOCAL LENGTHS................................................. 94

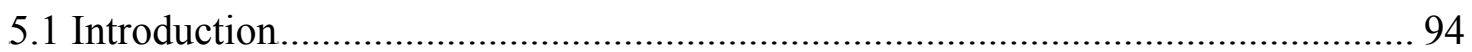

5.2 Dual Frequency Effect............................................................................... 96

5.3 Device Structure and Experimental Methods .................................................. 98

5.4 Experimental Results and Discussions ........................................................ 99

5.5 Numerical Calculations and Simulations...................................................... 104

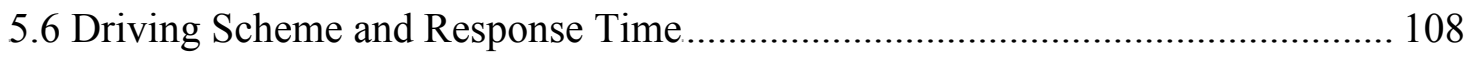

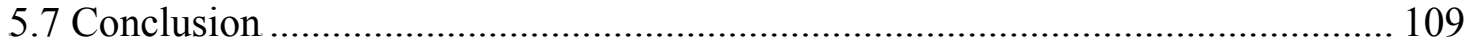

CHAPTER 6: ANISOTROPIC AND ISOTROPIC LIQUID-CRYSTAL GELS ........... 111

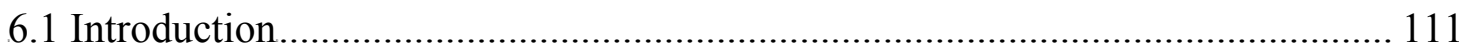

6.2 Normal-Mode Anisotropic Liquid-Crystal Gels............................................ 112

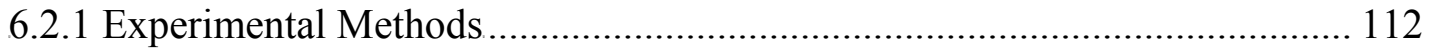

6.2.2 Experimental Results and Discussions ............................................... 113

6.2.3 Conclusion ..................................................................................... 120

6.3 Dual-Frequency Liquid Crystal Gels with Submillisecond Response Time ........ 120

6.3.1 Experimental Methods......................................................................... 120

6.3.2 Experimental Results and Discussions ............................................... 122

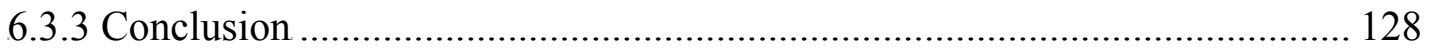

CHAPTER 7: FAST-RESPONSE AND SCATTERING-FREE POLYMER NETWORK

LIQUID CRYSTALS FOR INFRARED LIGHT MODULATORS ........................... 130

7.1 Introduction: Fast-Switching Liquid Crystal Devices ................................... 130

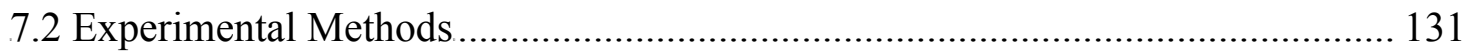


7.3 Experimental Results and Discussions ................................................... 134

7.4 Numerical Calculations and Simulations...................................................... 139

7.5 Methods of Reducing Operating Voltage ...................................................... 143

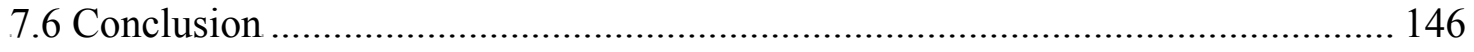

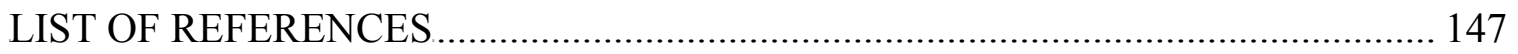




\section{LIST OF FIGURES}

Figure 1.1: Thermotropic liquid crystal: Liquid crystalline mesophases between the solid

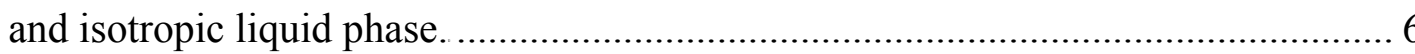

Figure 1.2: Geometrical structures of liquid crystal molecules................................... 7

Figure 1.3: Illustration of molecular alignments of liquid crystals: Nematic, Cholesteric,

Smectic A, and Smectic C. ........................................................................ 8

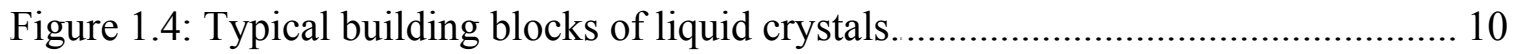

Figure 1.5: Coordinate system defining the microscopic order parameter of a nematic liquid crystal molecule. $\hat{n}$. is the director, the average direction of liquid crystal

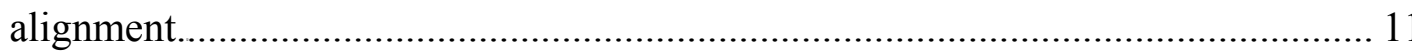

Figure 1.6: The three types of deformation occurring in nematics. The figure shows how each type may be obtained separately be suitable glass walls

Figure 1.7: Schematic depiction of the polymer-dispersed liquid crystal material at (a) voltage-off state and (b) voltage-on state. 15

Figure 1.8: (a) Fabrication of an inhomogeneous PDLC using a patterned photomask. (b) Optical polarizing microscope photos of two PDLC samples with gradient droplet size distribution. LC composite: $70 \%$ NOA81 and 30\% E7. $I=350 \mathrm{~mW} / \mathrm{cm}^{2}$. for 20

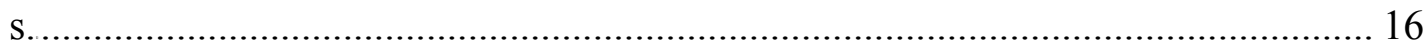

Figure 1.9: Chemical structures of (a) acrylate monomer and (b) acrylate polymer. R represents for any atom or group of atoms.

Figure 1.10: Illustration of free radical vinyl polymerization. 20 
Figure 1.11: Chemical structures of (a) one kind of methacrylate monomer and (b) PMMA which is made by free radical vinyl polymerization from the monomer methyl methacrylate.

Figure 1.12: The microscopic photo of PNLC cell. The chain-like polymer networks are along the rubbing direction. 21

Figure 1.13: Schematic of the PSCOF structure. 23

Figure 1.14: Schematic of the internal structure of a PSCOF device. $d$ is the cell thickness, which depends on the size of bead spacers used, and $L$ and $A$ are the average lateral and transverse dimensions of the LC volume. $B$ dimension characterizes the areas where the polymer-LC interface binds to the substrate on the opposite side.

Figure 1.15: Scanning electron micrographs of the polymer film structure taken after removing the substrate close to the LC and washing away the LC. The volume filled with LC (a) and the areas that were in contact with the opposite substrate (b) are indicated. (A) At 20\% LC concentration, grooves or elongated LC droplets form along the rubbing direction after the phase separation. (B) The formation of nearly uniform parallel layers of LC and polymer (that is, a PSCOF structure) is confirmed for $40 \% \mathrm{LC}$ at the same rate of phase separation as in (A). At a faster rate of phase separation, PDLCs are formed. (Courtesy of Dr. S. Kumar of Kent State University).

Figure 2.1: LC blazed-grating beam defector by using a glass substrate with a saw-tooth surface structure. PI: polyimide; ITO: indium-tin-oxide; PMMA: poly(methyl methacrylate). 28 
Figure 2.2: LC blazed grating by using an electric field with saw-tooth distribution. The liquid-crystal director varies only with $x$

Figure 2.3: Method for fabrication PNLC prism grating. The patterned photomask has periodic spatially varying grating structures.

Figure 2.4: Microscope photographs of the PNLC gratings taken at $V=0,1.5$, and 10 $\mathrm{V}_{\text {rms. }}$ The LC used is E48 and cell gap $d=8 \mu \mathrm{m}$. 33

Figure 2.5: The experimental setup used for studying the diffraction patterns and measuring the electro-optical properties of the PNLC prism grating. $\lambda=543.5 \mathrm{~nm} .34$

Figure 2.6: Photographs of the diffraction patterns observed in a dark room at $V=0,1.5$, and $2 \mathrm{~V}_{\text {rms. }}$ The PNLC prism grating has $300 \mu \mathrm{m}$ grating stripe. 35

Figure 2.7: Voltage-dependent relative transmittance of the $0^{\text {th }}$. (circles) and the $1{ }^{\text {st }}$. (triangles) order diffracted beams. The grating stripe width is $300 \mu \mathrm{m} . . . \ldots \ldots \ldots \ldots \ldots . . . . . .36$

Figure 3.1: (a) Schematic diagram of a binary amplitude/phase Fresnel lens. In the amplitude lens every other zone is opaque, while in the phase lens they are all transparent with an induced $\pi$ phase shift between the neighboring zones. (b) The phase shift induced by a binary phase Fresnel lens as a function of $R^{2}$. The period of this function is $2 R_{1}{ }^{2}$. The amplitude of the transmittance function is unity.

Figure 3.2: Diffraction of a plane wave at a Fresnel zone plate.................................. 42

Figure 3.3: Phase profile of a diffractive lens with multiple discrete phase levels......... 46

Figure 3.4: Efficiency of a diffractive lens as a function of the number of phase levels. 47. Figure 3.5: Focal spectrum of a diffractive lens as number $L$ of phase levels. 48 
Figure 3.6: Left: method for fabricating nano-PDLC Fresnel lens. Right: the photomask showing Fresnel zone structure. The open circles indicate the inhomogeneous droplet size distribution.

Figure 3.7: The experimental setup for studying the focusing properties of the PDLC Fresnel lens. $\mathrm{L}_{1}$ : focal length $50 \mathrm{~mm}, \mathrm{~L}_{2}$ : focal length $250 \mathrm{~mm}$, and P: $30 \mu \mathrm{m}$ pinhole. 52

Figure 3.8: Spot intensity profiles measured by the CCD camera under different conditions. (a) Without sample, (b) with sample at $V=0$, and (c) $V=180 \mathrm{~V}_{\text {rms. }}$ The diameter of the Fresnel zone plate is $1 \mathrm{~cm}$ and the focal length is $50 \mathrm{~cm}$. 53

Figure 3.9: Imaging and focusing properties of the PDLC Fresnel lens recorded by a CCD camera. (a) without sample, (b) CCD at $25 \mathrm{~cm}$ from sample, and (c) CCD at focal point. 55

Figure 3.10: Voltage-dependent diffraction efficiency of the PDLC Fresnel lens. The LC employed is E48, cell gap $d=15 \mu \mathrm{m}$ and $\lambda=633 \mathrm{~nm}$. 56

Figure 3.11: Schematic representation of the photomask patterns and the corresponding LC arrangement of the PNLC Fresnel lens. 60

Figure 3.12: Microscope images of the PNLC cell at (a) $V=0$, (b) $4 \mathrm{~V}_{\text {rms }}$, (c) $7 \mathrm{~V}_{\text {rms, }}$, and (d) $10 \mathrm{~V}_{\mathrm{rms}}$. The LC cell is sandwiched between crossed polarizers. 61

Figure 3.13: The observed laser beam images: (a) without LC sample, (b) with sample at $V=0$, and (c) with LC sample at $8 \mathrm{~V}_{\text {rms. }} \lambda=633 \mathrm{~nm}$. 64

Figure 3.14: The observed laser beam images (a) at $V=0$ and (b) at $8 \mathrm{~V}_{\text {rms. }} \lambda=633 \mathrm{~nm}$. The CCD camera was $27 \mathrm{~cm}$ away from the sample. 
Figure 3.15: Beam intensity profile measured by the CCD camera at $V=8 \mathrm{~V}_{\text {rms. }}$ The diameter of the Fresnel zone plate is $1 \mathrm{~cm}$ and the focal length is $50 \mathrm{~cm}$.

Figure 3.16: Beam intensity profiles at (a) $V=0$, (b) $8 \mathrm{~V}_{\mathrm{rms}}$, and (c) $20 \mathrm{~V}_{\mathrm{rms}}$. The diameter of the Fresnel zone plate is $1 \mathrm{~cm}$ and the focal length is $50 \mathrm{~cm}$. 66

Figure 3.17: The voltage-dependent diffraction efficiency of the PNLC Fresnel lens. LC:

MLC-6252, cell gap $d=5 \mu \mathrm{m}$, and monomer concentration $\mathrm{c}=5 \%$.

Figure 3.18: Schematic representation of the fabrication process of the PSCOF Fresnel lens. 69

Figure 3.19: Cross-sectional model of the PSCOF Fresnel lens. 70

Figure 3.20: Microscope images of the PSCOF Fresnel lens cell at $V=0$. The sample is sandwiched between two crossed polarizers.

Figure 3.21: Microscope images of the PSCOF Fresnel lens at (a) $V=0$, (b) $4 \mathrm{~V}_{\text {rms }}$, (c) 6 $\mathrm{V}_{\text {rms, }}$, and (d) $16 \mathrm{~V}_{\text {rms. }}$ The LC cell is sandwiched between two crossed polarizers. Cell gap $d=16 \mu \mathrm{m}$ 73

Figure 3.22: Image properties of the PSCOF Fresnel lens recorded by a CCD camera at (a) $5 \mathrm{~V}_{\text {rms }}$ and (b) $12 \mathrm{~V}_{\text {rms }}$ 75

Figure 3.23: The voltage-dependent diffraction efficiency of the PSCOF Fresnel lens.

The diameter of the Fresnel zone plate is $1 \mathrm{~cm}$ and the focal length is $50 \mathrm{~cm} \ldots \ldots \ldots . . .76$

Figure 3.24: Beam intensity profile measured by the CCD camera at (a) $V=5 \mathrm{~V}_{\text {rms }}$, and (b) $12 \mathrm{~V}_{\text {rms. }}$ The diameter of the Fresnel zone plate is $1 \mathrm{~cm}$ and the focal length is 50 $\mathrm{cm}$. 77 
Figure 4.1: Procedures for fabricating the spherical LC lens: (a) deposit ITO on a concave glass lens, (b) fill the sag area with polymer, and (c) assemble the LC lens cell with another flat glass substrate.

Figure 4.2: Interferograms of the circular LC lens at two different operating voltages: (a) $V=0$, (b) $V=25 \mathrm{~V}_{\text {rms. }}$ The lens aperture $D=6 \mathrm{~mm}$. The polarizers are crossed. The rubbing direction of the cell is oriented at $45^{\circ}$ with respect to the fast axis of the linear polarizer. LC used is UCF-2 and cell gap $d=40 \mu \mathrm{m}$. 82

Figure 4.3: CCD images of the measured He-Ne laser beam intensity profile at $V=0,23$, and $35 \mathrm{~V}_{\text {rms }}$, respectively 83

Figure 4.4: Imaging behavior of the LC lens at (a) $V=0$ and (b) $V=30 \mathrm{~V}_{\mathrm{rm}}$ 85

Figure 4.5: Voltage-dependent focal length of the LC lens. Lens aperture $D=6 \mathrm{~mm}, \mathrm{LC}$ : UCF-2, cell gap $d=40 \mu \mathrm{m}$ and $\lambda=633 \mathrm{~nm}$. Closed squares and open circles represent the experimental and simulation results, respectively 86

Figure 4.6: The LC directors distribution of a homogeneous cell at various normalized voltage $\left(V / V_{t h}\right)$. 90

Figure 5.1: Schematic representation of the structure of a DFLC microlens array 99

Figure 5.2: Microscope images of the DFLC microlens array at (a) $V=0$, (b) $28 \mathrm{~V}_{\mathrm{rms}}$, (c) $40 \mathrm{~V}_{\text {rms }}$ and (d) $200 \mathrm{~V}_{\text {rms. }}$ The LC cell is sandwiched between crossed polarizers. The rubbing direction of the cell is oriented at $45^{\circ}$ with respect to the fast axis of the linear polarizer. 101

Figure 5.3: The experimental setup for studying the focusing properties of the microlens array. $\mathrm{L}_{1}$ : focal length $50 \mathrm{~mm}, \mathrm{~L}_{2}$ : focal length $250 \mathrm{~mm}$, and P: $30 \mu \mathrm{m}$ pinhole. The polarization of the incident light is parallel to the rubbing direction. 102 
Figure 5.4: CCD images of the DFLC microlens array (left) and the corresponding 3-D light intensity profiles (right) at (a) 0, (b) $50 \mathrm{~V}_{\text {rms }}$, (c) $150 \mathrm{~V}_{\text {rms }}$ and (d) $200 \mathrm{~V}_{\text {rms. }} 103$

Figure 5.5: Voltage-dependent average refractive index of dual-frequency liquid crystal used in the experiment. Solid line and open circles represent the experimental and simulation results, respectively. $\lambda=633 \mathrm{~nm}$

Figure 6.1: Voltage-dependent normalized transmittance of the LC gels at different monomer concentrations in a $10 \mu \mathrm{m}$ cell. From top to bottom, $c=1.0,0.8,0.6$, and 0.4 wt $\%$ 114

Figure 6.2: Schematic representation of the operating principle of LC gels. (a) Voltageoff state and (b) voltage-on state. The arrows in the substrates indicate the rubbing directions 115

Figure 6.3: The spectral bandwidth of a $10 \mu \mathrm{m}$ LC gel. Upper trace: $V=10 \mathrm{~V}_{\text {rms; }}$ lower trace: $V=0$. A calcite polarizer was used to polarize the incident light.

Figure 6.4: Voltage-dependent normalized transmittance of the LC gels with monomer concentration of 0.4 wt. $\%$ at 30 (solid lines) and 100 (dashed lines) $\mu \mathrm{W} / \mathrm{cm}^{2}$. UV intensities.

Figure 6.5: A reflective display consists of a normal-mode LC gel, a quarter-wave film, and a reflector. (a) Voltage-off state and (b) voltage-on state. The arrows in the substrates indicate the rubbing directions.

Figure 6.6: Voltage-dependent normalized transmittance of the homogeneous-aligned DFLC gels at different monomer concentrations. Solid and gray lines are for $f=50$ $\mathrm{kHz}$ and $1 \mathrm{kHz}$, respectively, and polarization $\|$ to the cell rubbing direction. Dashed 
lines are for $c=5 \mathrm{wt} \%$ and $f=50 \mathrm{kHz}$, but polarization ${ }^{\wedge}$ to the cell rubbing direction. Cell gap $d=8 \mu \mathrm{m}, \lambda=633 \mathrm{~nm}$, and $T=22{ }^{\circ} \mathrm{C}$ 123

Figure 6.7: Schematic representation of the operating principle of homogeneous-aligned DFLC gels. (a) Voltage-off state and (b) voltage-on state. The arrows in the substrates indicate the rubbing directions.

Figure 6.8: Voltage-dependent normalized transmittance of the DFLC gels with monomer concentration of $5 \mathrm{wt} \%$ at $15 \mathrm{~V}_{\text {rms }}$ (solid lines), $8 \mathrm{~V}_{\text {rms }}$ (gray lines), and $4 \mathrm{~V}_{\text {rms. }}$. (dashed lines) bias voltage during UV illumination.

Figure 6.9: Voltage-dependent normalized transmittance of the no-alignment DFLC gels with monomer concentration of $5 \mathrm{wt} \%$ in an $8-\mu \mathrm{m}$ cell.

Figure 6.10: Schematic representation of the operating principle of no-alignment DFLC gels in a voltage-on state. The frequency of applied voltage is $50 \mathrm{kHz}$.

Figure 6.11: The spectral bandwidth of an 8- $\mu \mathrm{m}$ no-alignment DFLC gel. Upper trace: $V$ $=50 \mathrm{~V}_{\mathrm{rms}}$ at $f=1 \mathrm{kHz}$; lower trace: $V=50 \mathrm{~V}_{\mathrm{rms}}$ at $f=50 \mathrm{kHz}$ 128

Figure 7.1: Experimental setup for measuring the electro-optic properties of the reflective and transmissive LC and PNLC cells. The rubbing direction of the cell is at $45^{\circ}$ to the polarization axis of the incident light. The LC cell- 1 and detector 1 are for reflective mode and LC cell-2 and detector 2 are for transmissive mode. $\mathrm{PBS}=$ polarizing beam splitter. $\mathrm{P}=$ polarizer. $\lambda=1.55 \mu \mathrm{m}$.

Figure 7.2: Voltage-dependent reflectance of E44 (gray lines) and PNLC (dark lines) cells. From left to right, $c=5,7$, and $10 \mathrm{wt} \%$. The cell gap for the reflective cells is $5 \mu \mathrm{m} . \lambda=1.55 \mu \mathrm{m}$ and $T=21^{\circ} \mathrm{C}$ 
Figure 7.3: Voltage-dependent reflectance (a) and transmittance (b) of E44 (gray lines) and PNLC (dark lines) cells. The cell gap for the reflective and transmissive cells is 7.7 and $12.5 \mu \mathrm{m}$, respectively. $\lambda=1.55 \mu \mathrm{m}$ and $T=21^{\circ} \mathrm{C}$. 136

Figure 7.4: Measured phase decay time of the reflective (a) and transmissive (b) PNLC cells. Solid lines are experimental data and open circles are fitting results using $\delta(\mathrm{t})$ $=2 \pi \exp (2 t / \tau)$. In (a), $\tau=296 \mu \mathrm{s}$ and in (b) $\tau=755 \mu$ s. $T=21^{\circ} \mathrm{C}$ and $\lambda=1.55 \mu \mathrm{m}$.

Figure 7.5: Temperature-dependent LC response time (a) and birefringence (b) of the E44 transmissive PNLC cell. $D=12.5 \mu \mathrm{m}$. Closed circles are the measured response time and open circles are the corresponding phase change.

Figure 7.6: Voltage-dependent average refractive index of dual-frequency liquid crystal used in the experiment. Solid line and open circles represent the experimental and simulation results, respectively. $\lambda=1.55 \mu \mathrm{m}$ 140

Figure 7.7: The simulation results of E44 (a) and PNLC (b) cells. Solid lines are experimental data and open circles are fitting results. $T=21^{\circ} \mathrm{C}$ and $\lambda=1.55 \mu \mathrm{m} .142$

Figure 7.8: Voltage-dependent reflectance PNLC cells cured with different bias voltage.

The cell gap for the reflective cells is $8 \mu \mathrm{m} . T=21^{\circ} \mathrm{C}$ and $\lambda=1.55 \mu \mathrm{m}$. 144

Figure 7.9: The simulation results of PNLC cell with different $\Delta n$ (a) and $\Delta \varepsilon$ (b). The fitting parameters: $k_{11}=4800 \mathrm{pN}$, and $k_{33}=50000 \mathrm{pN} . \lambda=1.55 \mu \mathrm{m}$. 145 


\section{LIST OF TABLES}

Table 4.1 Physical parameters of UCF-2 for simulation .......................................... 91

Table 5.1 The physical parameters of our homemade dual-frequency liquid crystal used

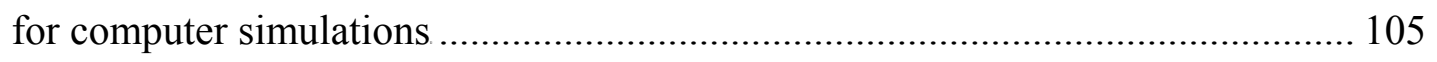

Table 7.1 Physical parameters of E44 for simulation ............................................. 140

Table 7.2 Physical parameters of PNLC sample for simulation................................. 141 


\section{LIST OF ABBREVIATIONS}

\begin{tabular}{|c|c|}
\hline $3-\mathrm{D}$ & Three-Dimensions, Three-Dimensional \\
\hline $5 \mathrm{CB}$ & 4-n-pentyl-4'-cyanobiphenyl \\
\hline AFLC & Antiferroelectric Liquid Crystal \\
\hline BAB-6 & 4,4[6-acyloyloxy)-hexyloxy]-1,1-biphenylene \\
\hline $\mathrm{CCD}$ & Charge Coupled Device \\
\hline $\mathrm{CR}$ & Contrast Ratio \\
\hline CREOL & Center for Research and Education in Optics and Lasers \\
\hline $\mathrm{DE}$ & Diffraction Efficiency \\
\hline DFLC & Dual Frequency Liquid Crystal \\
\hline FLC & Ferroelectric Liquid Crystal \\
\hline FPCE & Florida Photonics Center of Excellence \\
\hline FZP & Fresnel Zone Plate \\
\hline GRIN & Gradient-Index \\
\hline $\mathrm{He}-\mathrm{Ne}$ & Helium-Neon \\
\hline HPDLC & Holographic Polymer-Dispersed Liquid Crystal \\
\hline $\mathrm{Hz}$ & Hertz \\
\hline IP & Integral Photography \\
\hline IPS & In-Plane-Switching \\
\hline IR & Infrared \\
\hline ITO & Indium-tin-oxide \\
\hline
\end{tabular}




\begin{tabular}{ll} 
kHz & Kilo-Hertz \\
LC & Liquid Crystal \\
MBBA & (4-methoxybenzylidene)-4'-butylbenzenamine \\
MEMS & Microelectromechanical Systems \\
NOA & Norland Optical Adhesives \\
OPA & Optical Phased Array \\
PI & Polyimide \\
PIPS & Polymerization-Induced Phase Separation \\
PDLC & Polymer-Dispersed Liquid Crystal \\
PMMA & Poly(methyl methacrylate) \\
PNLC & Polymer Network Liquid Crystal \\
PSCOF & Phase-Separated Composite Film \\
PSLC & Polymer-Stabilized Liquid Crystal \\
PBS & Polarizing Beam Splitter \\
QWP & Quarter Wave Plate \\
SIPS & Solvent-Induced Phase Separation \\
SSFLC & Surface-Stabilized FLC \\
TIPS & Thermally Induced Phase Separation \\
UV & Ultraviolet \\
VOA & Variable Optical Attenuator \\
\hline
\end{tabular}




\section{LIST OF PUBLICATIONS}

1. Y. H. Fan, H. Ren, X. Liang, H. Wang, and S. T. Wu, "Liquid crystal microlens arrays with switchable positive and negative focal lengths," (Submitted).

2. H. Ren, Y. H. Lin, Y. H. Fan, and S. T. Wu, "Polarization-independent phase modulation using a polymer-dispersed liquid crystal," Applied Physics Letters 86, 141110 (2005).

3. H. Ren, Y. H. Fan, Y. H. Lin, and S. T. Wu, "Tunable-focus microlens arrays using nanosized polymer-dispersed liquid crystal droplets," Optics Communications 247, 101-106 (2005).

4. Y. H. Fan, H. Ren, X. Liang, Y. H. Lin and S. T. Wu, "Fast-response dual frequency liquid crystal gel," in MRS 2004 Fall Meeting, Boston, MA, November 2004.

5. Y. H. Fan, H. Ren, X. Liang, and S. T. Wu, "Tunable-focus liquid crystal microlens arrays for display applications," in IEEE LEOS 2004 Annual Meeting, Rio Grande, Puerto Rico, November 2004.

6. H. Ren, J. R. Wu, Y. H. Fan, Y. H. Lin, and S. T. Wu, "Hermaphroditic liquid crystal microlens," Optics Letters 30, 376-378 (2005).

7. Y. H. Wu, Y. H. Lin, Y. Q. Lu, H. Ren, Y. H. Fan, J. R. Wu and S. T. Wu, "Submillisecond response variable optical attenuator based on sheared polymer network liquid crystal," Optics Express 12, 6377-6384 (2004). 
8. Y. H. Fan, H. Ren, X. Liang, Y. H. Lin and S. T. Wu, "Dual frequency liquid crystal gels with sub-millisecond response time," Applied Physics Letters 85, 24503 (2004).

9. H. Ren, Y. H. Lin, Y. H. Fan, and S. T. Wu, "In-plane switching liquid crystal gel for polarization independent light switch," Journal of Applied Physics 96, 3609-11 (2004).

10. H. Ren, Y. H. Fan, S. Gauza, and S. T. Wu, Tunable-focus flat liquid crystal spherical lens," Applied Physics Letters 84, 4789-91 (2004).

11. H. Ren, Y. H. Fan and S. T. Wu, "Liquid crystal microlens arrays using patterned polymer networks," Optics Letters 29, 1608-1610 (2004).

12. Y. H. Fan, Y. H. Lin, H. Ren, S. Gauza and S. T. Wu, "Fast-response and scattering-free polymer network liquid crystals for infrared light modulators," Applied Physics Letters 84, 1233-5 (2004).

13. H. Ren, Y. H. Fan, S. Gauza and S. T. Wu, "Tunable microlens arrays using polymer network liquid crystal," Optics Communications 230, 267-71 (2004).

14. H. Ren, Y. H. Fan, and S. T. Wu, "Polymer network liquid crystal for tunable microlens arrays," Journal of Physics D: Applied Physics 37, 400-3 (2004).

15. H. Ren, Y. H. Fan. S. Gauza and S. T. Wu, "Tunable-focus cylindrical liquid crystal lens," Japanese Journal of Applied Physics 43, 652-3 (2004).

16. Y. H. Fan, Y. H. Lin, H. Ren, S. Gauza and S. T. Wu, "Fast-response polymer network liquid crystals for optical communications", SID Symposium Digest of Technical Papers 35, 1526-9 (2004). 
17. (Invited paper) Y. H. Fan, H. Ren, and S. T. Wu, "Electrically controlled lens and prism using nanoscale polymer-dispersed and polymer-networked liquid crystals," Proceedings of SPIE 5289, 63-73 (2004)

18. Y. H. Fan, H. Ren and S. T. Wu, "Switchable Fresnel lens using polymer-stabilized liquid crystals," Optics Express 11, 3080-6 (2003).

19. H. Ren, Y. H. Fan and S. T. Wu, "Tunable Fresnel lens using nanoscale polymerdispersed liquid crystals," Applied Physics Letters 83, 1515-7 (2003).

20. H. Ren, Y. H. Fan and S. T. Wu, "Prism grating using polymer-stabilized liquid crystal,” Applied Physics Letters 82, 3168-70 (2003).

21. Y. H. Fan, H. Ren and S. T. Wu, "Normal mode anisotropic liquid crystal gels," Applied Physics Letters 82, 2945-7 (2003).

22. A. Spadło, R. Dąbrowski, M. Filipowicz, Z. Stolarz, J. Przedmojski, S. Gauza, Y. H. Fan and S. T. Wu, "Synthesis, Mesomorphic and optical properties of isothiocyanatotolanes," Liquid Crystal 30, 191-8 (2003).

23. (Invited Paper) H. Ren, Y. H. Fan and S. T. Wu, “Anisotropic liquid crystal gels for display applications,” Proc. International Display Manufacturing Conference, pp. 29-32 (2003).

24. (Invited paper) C. H. Wen, Y. H. Fan, S. T. Wu, R. Dabrowski, O. Catanescu and L. C. Chien, "Side chain effects on high birefringence liquid crystals," Proceedings of SPIE 4658, 28-34 (2002).

25. T.-C. Ko, Y.-H. Fan, M.-F. Shieh, A. Y.-G. Fuh, C.-Y. Huang, M.-S. Tsai and I-M. Jiang, "Diffraction from polymer-stabilized cholesteric texture films," Japanese Journal of Applied Physics 40, 2255-8 (2001). 
26. A. Y.-G. Fuh, M.-S. Tsai, C.-R. Lee, Y.-H. Fan, "Dynamical studies of gratings formed in polymer-dispersed liquid crystal films doped with a guest-host dye," Physical Review E 62, 3702-7 (2000).

27. A. Y.-G. Fuh, M.-S. Tsai, Y.-H. Fan, C.-R. Lee, K.-Y Lo, "Dynamic studies of polymerization in liquid crystal-polymer mixtures," Journal of Applied Physics 86, 6652-6 (1999).

28. A. Y.-G. Fuh, M.-S. Tsai, C.-R. Lee, Y.-H. Fan, "Diffraction from a one-beam generated hologram on a polymer-dispersed liquid crystal film," Optics Communications 167, 53-6 (1999). 


\section{CHAPTER 1: INTRODUCTION}

\section{$\underline{1.1 \text { Motivation }}$}

Liquid crystal (LC) devices have impacted human lives in displays and photonics. In display area, LC has been widely used in digital watches, laptop and desktop computers, data projectors, and high-definition televisions. In photonic area, LC-based adaptive optics has been explored for information processing, optical interconnections, integrated optics, and optical communications due to their tunable optical properties. Enormous amount of research has been devoted to exploring other new exciting applications.

In many optical systems such as lenses, polarizers, gratings and prisms, a desirable feature is to dynamically control those components. Let us take Fresnel lens as an example, continuously tunable diffraction efficiency would eliminate the extra need for external amplitude spatial light modulators.

An electrically tunable LC lens is a potential candidate for optical information processing, optical interconnection, beam deflector, and three-dimensional (3-D) display systems. Among the various applications, LC lenses and beam steering devices have been

developed. $^{1-12}$. Large birefringence and low control voltage distinguish nematic liquid crystals from other electro-optical materials. Several methods have been addressed using a liquid crystal. For those devices using liquid crystal, the problem of slow response time 
needs to be solved. Therefore, it would be highly desirable to improve the material and fabrication method to enhance the response time for the LC devices.

In this dissertation, we have developed novel fabrication methods of tunable photonic devices with fast switching speed. With the use if liquid crystal/polymer composites and dual-frequency liquid crystal, the response time can be improved significantly.

Liquid crystal/polymer composites could appear in different forms depending on the fabrication conditions. Polymer-dispersed liquid crystals (PDLCs) are composite materials consisting of liquid crystal droplets dispersed in a polymer matrix. Usually, they have droplet sizes on the order of micrometers, so that the light scattering dominates in the visible region. For imaging processing and beam steering applications, light scattering causes optical loss in transmission. It is desired to control the light scattering for the operating wavelength.

By increasing the speed of phase separation process, the nano-sized PDLC can be obtained. For optical communication technology, the nanoscale PDLC is optically isotropic with respect to the incident light and highly transparent in the voltage-off state. In a high-voltage state, the nano-sized droplets are reoriented by the electric field resulting in a phase change.

The nanoscale LC droplets are randomly distributed, so that the devices are polarization independent. Due to the nanoscale droplet sizes, the response time is fast (in sub-millisecond range), however, the operating voltage is higher than $100 \mathrm{~V}_{\text {rms. }}$ To lower the driving voltage, polymer-network liquid crystal (PNLC) has been developed. In a PNLC, a different kind of monomer with rod-like structure is used. The LC concentration 
exceeds $95 \%$ so that the operating voltage is low ( $\left.10 \mathrm{~V}_{\mathrm{rms}}\right)$. However, the PNLC devices are polarization dependent. To overcome this drawback, we could stack two cells with orthogonal alignment directions.

A device using phase-separated composite film (PSCOF) can be operated at low voltage as well, since the LC and polymer are separated completely to form two layers. Without multi-domain formed in the LC cell, this kind of device has no light scattering. With different fabrication methods, liquid crystal blazed grating and Fresnel lens has been demonstrated using liquid crystal/polymer composites. Fresnel zone plates are very attractive devices due to their focusing abilities and compactness. In comparison with the refractive lenses, the Fresnel lens is simple to fabricate. This advantage manifests when the lens aperture size becomes large.

Besides Fresnel lens, a critical need for imaging and display applications is to develop a zooming optical system with continuously tunable focal length. For instance in a conventional zoom lens, the zooming effect is achieved by controlling the lens separation along the optical axis. This kind of system is bulky and requires extra mechanical elements for movement. An electrically controllable liquid crystal lens is desired to keep the system compact and low cost. Without complicated electrode design, we demonstrate a new liquid crystal lens. The focal length is tunable by applying a uniform electric field. A huge tunable range from $0.6 \mathrm{~m}$ to infinity is achieved.

Microlens arrays have been investigated for various applications. In 3-D displays, a system with variable focal length gives an observer an enhanced perception of depth, since the resolution of a real or virtual image is improved by dynamically adjusting the image plane. This method is especially helpful in displaying 3-D animated images. A LC 
microlens array whose focal length can be switched from positive to negative or vise versa by the applied voltage is demonstrated.

Controllable light scattering is a special feature for LC/polymer composites. This attractive feature has been used in light shutters and switchable windows. It is important to control the devices and developing novel fabrication methods to have high contrast ratio and fast switching speed. Unlike PDLC, for modulating unpolarized light, polymer network liquid crystal (also called LC gel) systems can have anisotropic light scattering behavior. It is also desired to control the scattering behavior sensitive or insensitive to light polarization. The use of a dual-frequency liquid crystal and special driving scheme leads to a sub-millisecond response time.

The polymer network technique is attractive due to its fast switching speed. It has been introduced to decrease the response time of an in-plane-switching (IPS) LCD from $\sim 90 \mathrm{~ms}$ in pure liquid crystal to $\sim 10 \mathrm{~ms}$ in $2 \%$ polymer network liquid crystal composites. However, the severe light scattering reduces the transmission and degrades the image quality. For laser beam steering application, a growing interest is to develop compact and lightweight laser beam scanning technologies for replacing the bulky and heavy gimbal mirror systems. Many solid state/micro-component technologies have been proposed to enable tactical laser communications and infrared countermeasure, such as optical MEMs, patterned liquid crystals, diffractive micro-optics and photonic crystals. The goal is to build small, ultra-light, rapidly steered laser beam sub-systems. Incorporating this increased functionality into new weapons systems will be accomplished through the refinement and integration of these microstructure technologies. For the approach of using a liquid crystal-based optical phased array (OPA) as electrically reconfigurable 
refractive element. The major challenge is to develop a fast-response and scattering-free polymer network liquid crystal (PNLC) light modulator. The goal is to achieve $2 \pi$ phase change in $1 \mathrm{~ms}$ at $\lambda=1.55 \mu \mathrm{m}$.

\subsection{Liquid Crystal}

The liquid crystalline state was discovered in 1888, when Reinitzer and Lehmann investigated some esters of cholesterol. The field of liquid crystal then becomes increasingly attractive in the following decades. It is commonly known that matters exist in three forms; solid, liquid, and gas. Solid could be either crystalline or amorphous. Crystals exhibit a long range molecular order. The molecules have a regular arrangement of over a large distance compared to molecular dimensions. When a crystalline solid is heated, it transforms to isotropic liquid at the melting point. Isotropic liquid does not have a long range order. Liquid crystal materials do not directly pass from crystalline solid to isotropic liquid but adopt an intermediate structure which flows like a liquid but still possesses the anisotropic physical properties similar to crystalline solids. Therefore, condensed matter which exhibit intermediate thermodynamic phases between the crystalline solid and the isotropic liquid state are now called liquid crystals or mesophases (Figure 1.1). If transitions between the phases are caused by temperature, they are called thermotropic. In blends of different components phase transitions may also depend on concentration, and these liquid crystals are called lyotropic. While thermotropics are presently mostly used for technical applications, lyotropics are important for biological systems, e.g. membranes. 

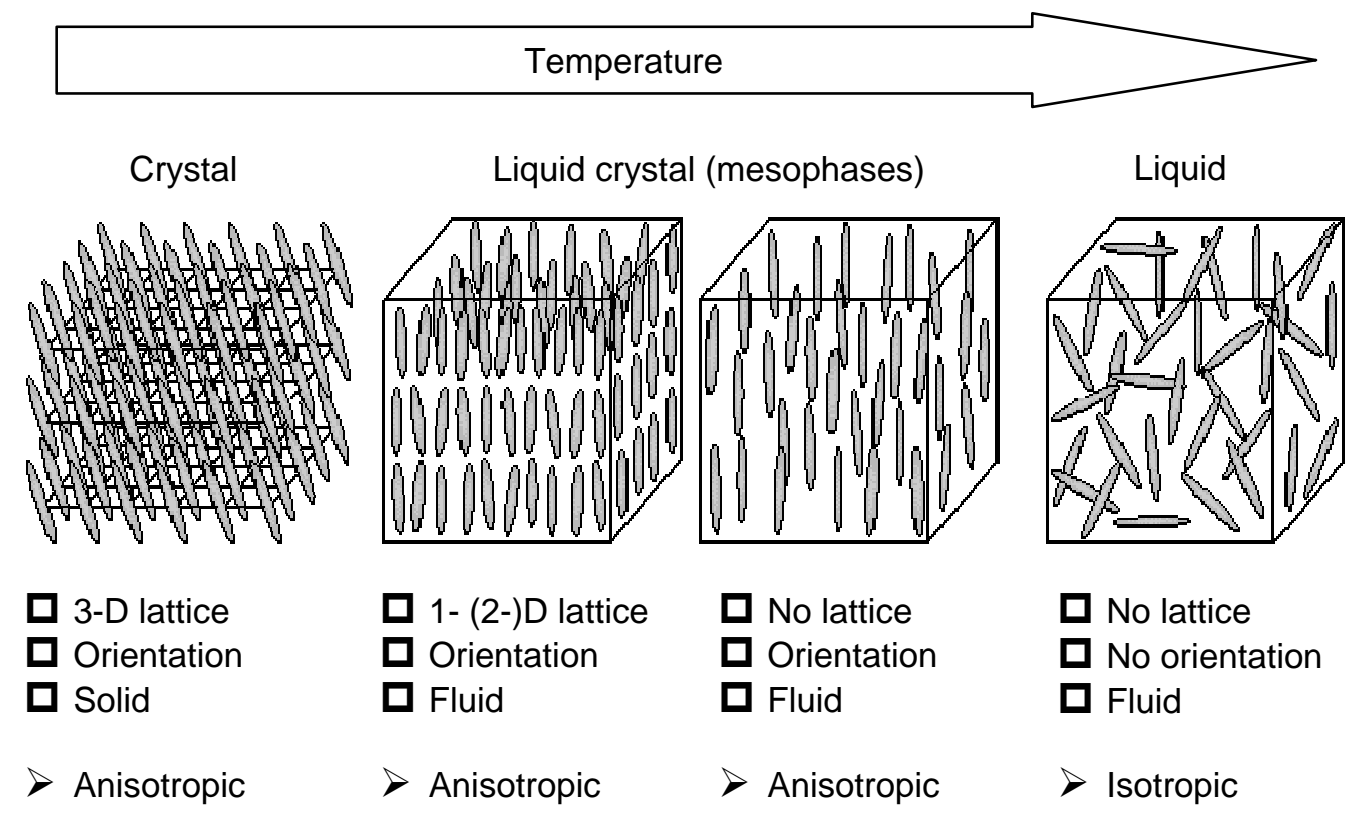

Figure 1.1: Thermotropic liquid crystal: Liquid crystalline mesophases between the solid and isotropic liquid phase.

Thermotropic liquid crystals are the most widely used and extensively studied class of liquid crystals. Considering the geometrical structure of the molecules, the thermotropic liquid crystals are usually divided into "rod-like", "disk-like", and "lathlike" organic molecules, sketched in Figure 1.2, which tend to align themselves with a long range order due to anisotropic intermolecular forces. The rod-like liquid crystals (also called calamitic liquid crystals) are the most common materials for optical applications. In a 1922 publication, a French scientist named Georges Friedel classified the rod-like liquid crystal phases as nematic, cholesteric, and smectic, shown in Figure1.3, based on the amount of structure in the liquid crystal material - in both the orientation of the molecules and the positional relationships between the molecules. 

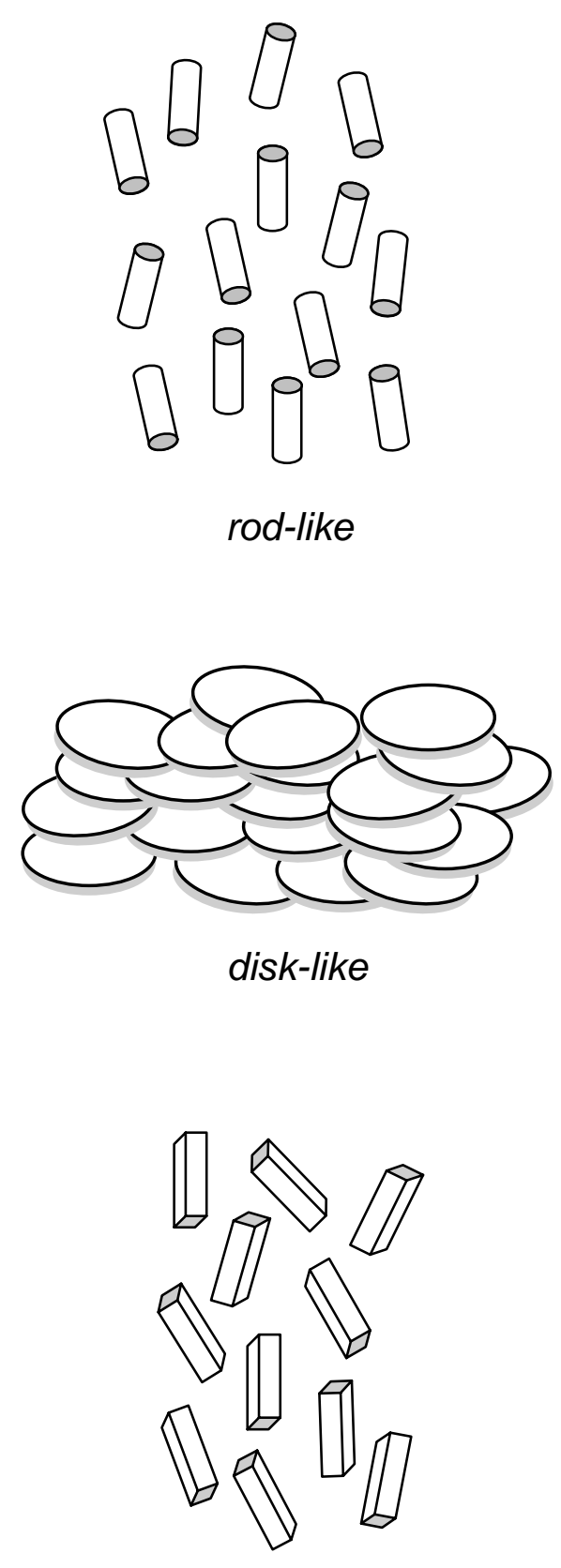

lath-like

Figure 1.2: Geometrical structures of liquid crystal molecules. 
In the simplest case the molecules possess only orientational but no positional long range order. Liquid crystals of that type are called nematic (from Greek $v \varepsilon \mu o \sigma=$ thread), because of the thread-like formations which can be seen under a polarizing microscope. The direction of preferred alignment can be described by a unit vector, the so-called nematic "director" $\widehat{n}$. In practice, the orientation of individual molecules differs significantly from that direction, and the director must be more correctly defined as the symmetry axis of the orientational distribution. In nematics the distribution function is rotationally symmetric around the director, i.e. they are uniaxial.

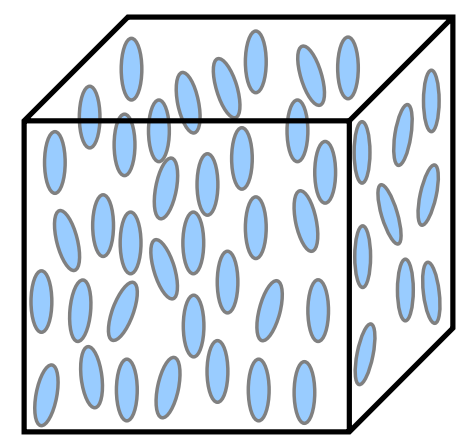

Nematic

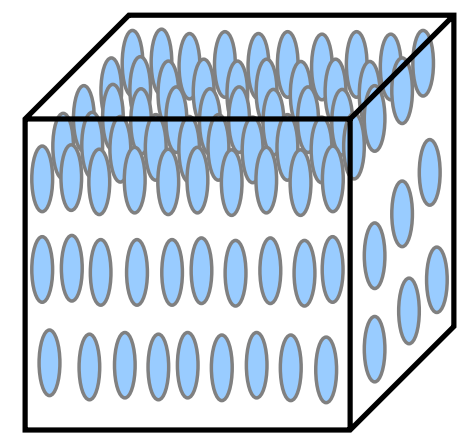

Smectic A

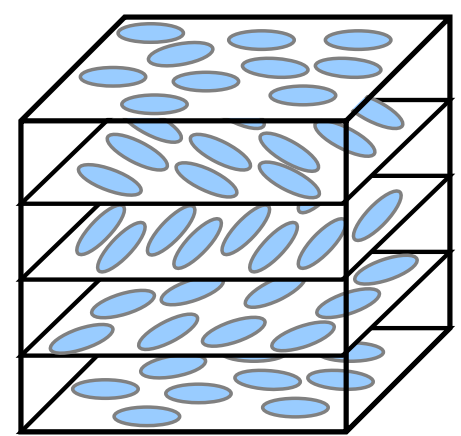

Cholesteric

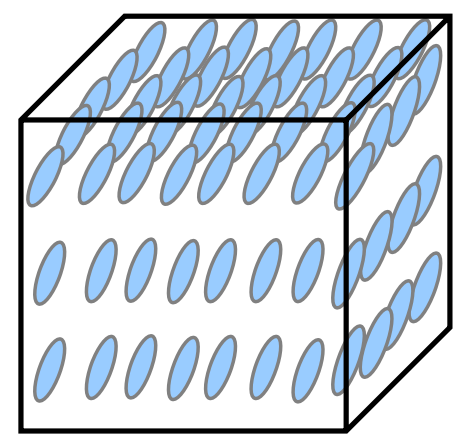

Smectic C

Figure 1.3: Illustration of molecular alignments of liquid crystals: Nematic, Cholesteric, Smectic A, and Smectic C. 
Cholesteric (or chiral nematic) liquid crystal resemble nematic liquid crystal in all physical properties except that the molecules tend to align in a helical structure with the helical axis perpendicular to the director, as depicted in Figure 1.3. This helical structure is exploited in several ways in making modern flat-panel displays. The cholesteric liquid crystal (from cholesterol acetate, the first kind of this type) can be obtained by adding a chiral molecule (the molecules differ from their mirror image) to a nematic liquid crystal.

The smectic phases (from Greek $\sigma \mu \varepsilon \gamma \mu \alpha=$ soap), unlike nematics, are characterized by additional degrees of positional order. Generally, the molecules are arranged in layers in these mesophases, which can be considered as one-dimensional density waves. There are at least nine distinct smectic phases bearing the designations A, $\mathrm{B}, \mathrm{C}, \ldots, \mathrm{I}^{13}$. Here we only briefly discuss the representative structures of the smectic A and $\mathrm{C}$ phases, as shown in Figure 1.3. In smectic A (SmA) liquid crystals, the molecular orientation is perpendicular to the layers. In each layer the molecules are positionally random, but directionally ordered with their long axis normal to the plane of the layer. Similar to nematics, smectic-A liquid crystals are optically uniaxial. There is a rotational symmetry around the director axis. In the $\mathrm{SmC}$ phase, the director is tilted at an angle to the layer normal. It is optically biaxial. Both SmA and SmC show no positional order within the layers and therefore are often considered as two-dimensional liquids. The SmC phase of chiral molecules may form a helical structure which is denoted as $\mathrm{SmC}^{*}$. 


\subsection{Nematic Liquid Crystals}

Nematics are the most widely studied liquid crystals. The liquid crystal materials used in this dissertation are all nematics. It is necessary to address more details about the physical properties of nematic liquid crystal.

In Figure 1.4, the chemical structures for two of the most prominent representatives of rod-like molecules which exhibit liquid crystalline order are shown. MBBA was the first room temperature liquid crystal synthesized in 1969, whereas 5CB was the first member of the optically and chemically stable cyano-biphenyls, one of the most applicable families of liquid crystals, discovered in 1973. The transition temperatures between crystalline $(\mathrm{C})$, liquid crystalline $(\mathrm{N}=$ nematic, see below), and isotropic (I) state given in Figure 1.4 are in Celsius.

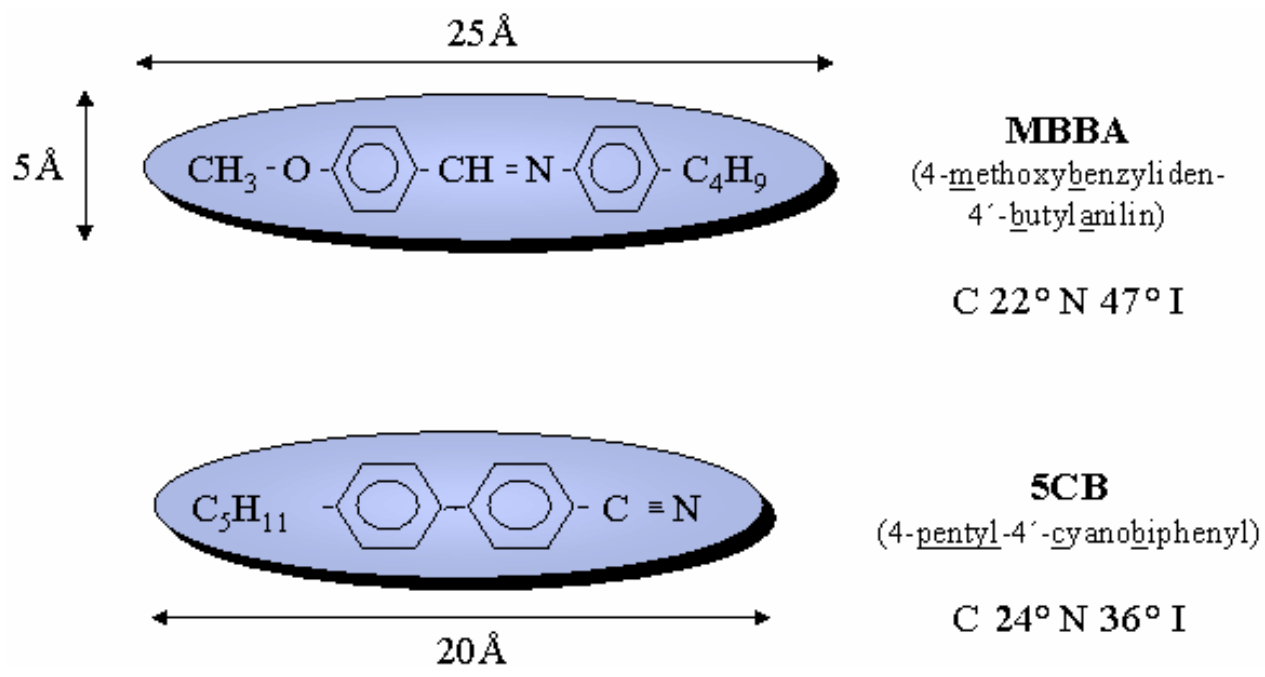

Figure 1.4: Typical building blocks of liquid crystals. 
The nematic phase has the lowest ordering of all the mesophases and, if present, precedes the transition to the isotropic liquid which occurs at the clearing point. As shown in Figure 1.3, nematics exhibit a long-range molecular orientational order but possesses not positional order. Generally, nematic molecules are centrosymmetric; their physical properties are the same in the $+\hat{n}$ and the optically uniaxial $-\hat{n}$ directions, i.e., $\hat{n}$ is an apolar vector. If the individual molecules carry a permanent electric dipole (such as polar nature), they will assemble in such a way that bulk dipole moment is vanishing. For the sake of simplicity in developing theories of the nematic phase, it is usually assumed that the molecules are cylindrically symmetric. The orientational order, so-called order parameter, in a uniaxial nematic can be described as a second rank tensor. ${ }^{14}$.

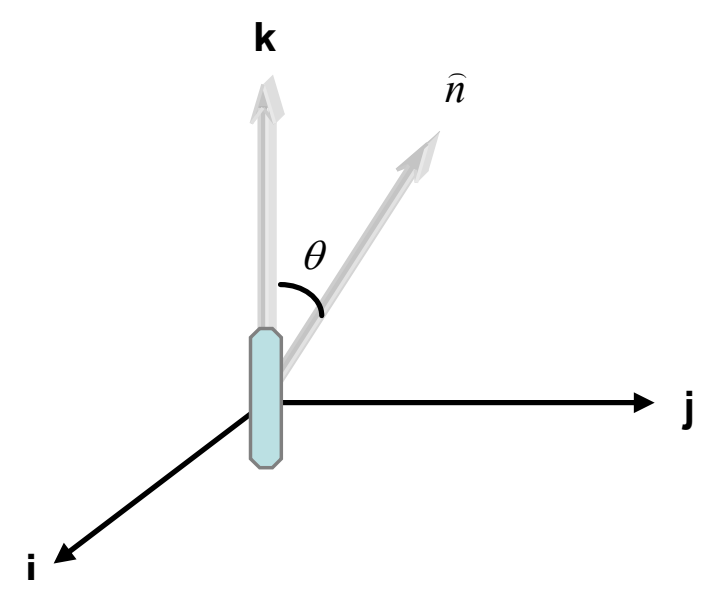

Figure 1.5: Coordinate system defining the microscopic order parameter of a nematic liquid crystal molecule. $\hat{n}$ is the director, the average direction of liquid crystal alignment. 

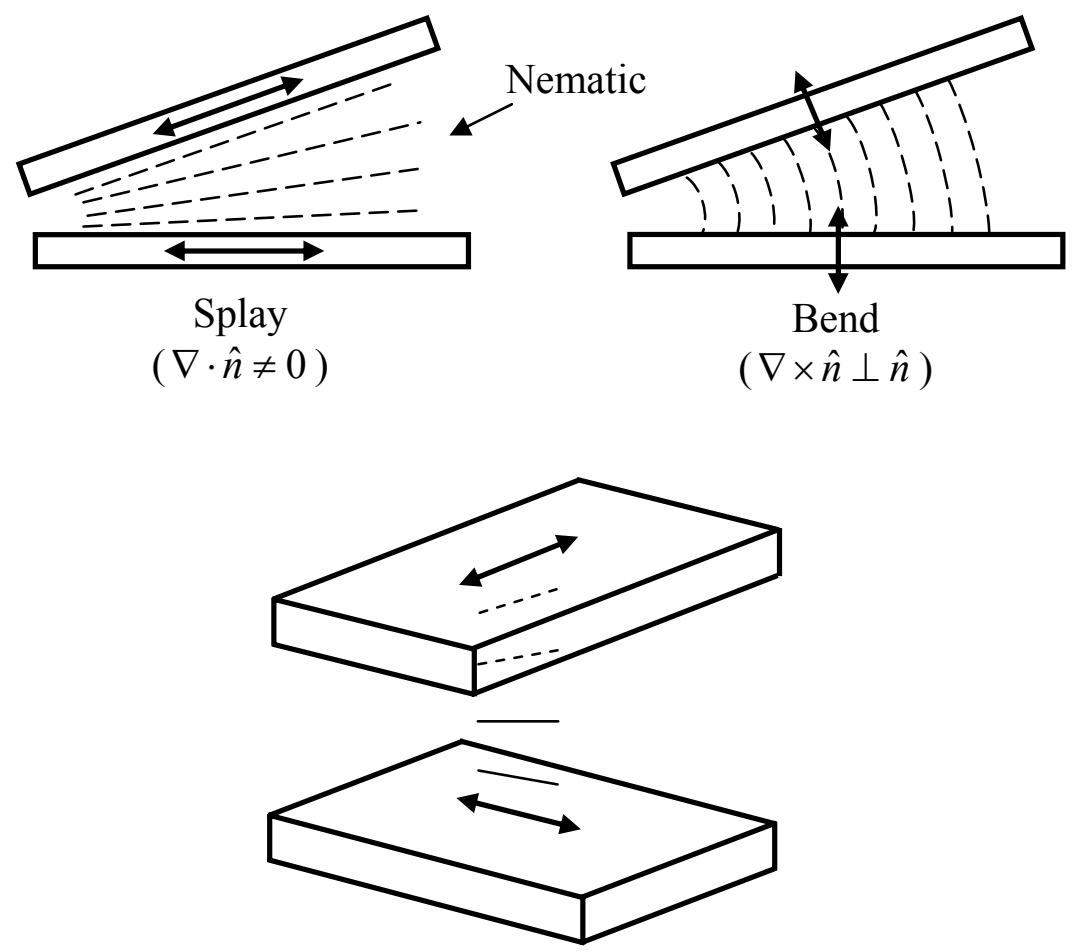

Twist

$(\nabla \cdot \hat{n} / / \hat{n})$

Figure 1.6: The three types of deformation occurring in nematics. The figure shows how each type may be obtained separately be suitable glass walls

If we use the long axis of the molecule as a reference and denote it as $\hat{k}$, the microscopic scalar order parameter $S$ is defined as follows:

$$
\begin{aligned}
S & =\frac{1}{2}\langle(\hat{k} \cdot \hat{n})(\hat{k} \cdot \hat{n})-1\rangle \\
& =\frac{1}{2}\left\langle 3 \cos ^{2} \theta-1\right\rangle
\end{aligned}
$$

Where $\theta$ is the polar angle made by the molecular axis with the director axis, shown in Figure 1.5. The angular brackets indicate a statistical average over the whole ensemble. 
This kind of order is usually termed long-range order. The scalar order parameter from Eq. (1.1) is sufficient for nematics which possesses cylindrical or rotational symmetry around the long axis $\hat{k}$. Clearly, $S$ goes to zero when an isotropic distribution is reached, and takes the value of 1 for all the molecules strictly parallel to one another. In the nematic phase, $S$ is in the intermediate values.

When an external field is present, such as stresses/constraints from the boundary surfaces, the director axis will be distorted. As a result, the order parameter will also vary spatially. In most cases, the characteristic length is much larger than the molecular size. Three typical examples are shown in Figure 1.6. ${ }^{14,15}$. The characteristic length is on the order of $1 \mu \mathrm{m}$, whereas the molecular dimension is on the order of at most a few tens of angstroms. Twist, splay, and bend are the three principal distinct director axis deformations in nematic liquid crystals. For twist deformations in liquid crystal, owing to the fluidity of the molecules, simply involve a rotation of the molecules in the direction of the torque; there is no translational displacement of the center of gravity of molecules, and thus the elastic energy involved is quite small. For splay and bend deformations, involving mainly changes in the director axis $\hat{n}$, they incur much less elastic energy change than the corresponding ones in ordinary solids. They involve flow of the liquid crystal, whereas the twist deformation does not. Since there are spatial changes in director $\hat{n}(r)$ for all the deformations, the basic parameters concerned about the deformation energies are various spatial derivatives. The free-energy densities (in unit of energy per volume) associated with three deformations are given by 


$$
\begin{aligned}
& \text { Splay: } F_{1}=\frac{1}{2} K_{11}(\nabla \cdot \hat{n})^{2} \\
& \text { Twist: } \quad F_{2}=\frac{1}{2} K_{22}(\hat{n} \cdot \nabla \times \hat{n})^{2} \\
& \text { Bend: } \quad F_{3}=\frac{1}{2} K_{33}(\hat{n} \times(\nabla \times \hat{n}))^{2}
\end{aligned}
$$

where $K_{11}, K_{22}$, and $K_{33}$ are the respective Frank elastic constants. ${ }^{16}$.

In general, the three elastic constants are different in magnitude. Typically, they are on the order of $10^{-11} \mathrm{~N}$ in MKS units (or $10^{-6}$ dyne in cgs units). For MBBA, $k_{11}, k_{22}$, and $k_{33}$ are $5.8 \times 10^{-12}, 3.4 \times 10^{-12}$, and $7.0 \times 10^{-12} \mathrm{~N}$, respectively. For almost all nematics $k_{33}$ is the largest, as a result of the rigid-rod shape of the molecules.

\subsection{Inhomogeneous Nanoscale Polymer-Dispersed Liquid Crystal (PDLC)}

Liquid crystal/polymer composites could appear in different forms depending on what fabrication condition is employed. As the polymer concentration exceeds $\sim 30-40 \%$, the PDLC sample can be prepared by four methods: encapsulation, ${ }^{17}$ thermally induced phase separation (TIPS), ${ }^{18}$. polymerization-induced phase separation (PIPS), and solventinduced phase separation (SIPS). ${ }^{19}$. The liquid crystal is separated out in the form of micron-sized droplets randomly distributed throughout the polymer. ${ }^{17,20}$. The droplet size can be controlled during the polymerization process and is usually comparable to the visible wavelength. PDLC can be used as a switchable window and light shutter.

A simple diagram illustrating the electro-optical effect of a PDLC shutter is shown in Figure 1.7. The polymer matrix material is optically isotropic and has a refractive index $n_{\mathrm{p}}$. The optical indices of these randomly oriented liquid crystal droplets, 
in the absence of an external alignment field, depend on the liquid crystal-polymer interaction at the boundary, and therefore assume a random distribution in the absence of an external field (cf. Figure 1.7(a)). The index mismatch $\left(n_{\mathrm{e}}>n_{\mathrm{p}}\right)$ between the LC droplets and polymer results in light scattering. If the cell thickness is much larger than the droplet size, the incident light will be scattered many times before emerging from the film. The PDLC cell looks like a ground glass. The degree of the off-state scattering depends on the size, birefringence and concentration of the liquid crystalline droplets, and the film thickness. Upon the application of a sufficiently strong external field, the directors in the droplets are reoriented along the field direction (Figure 1.7(b)). The system will become clear as the refractive index of the liquid crystal droplets is equal to $n_{\mathrm{o}}$ which is similar to the index of the polymer, $n_{\mathrm{p}}$. Due to the random distribution of directors in the LC droplets, these PDLC devices are independent of light polarization.

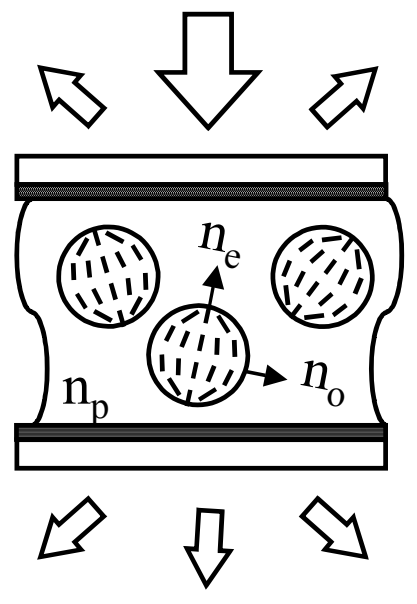

(a)

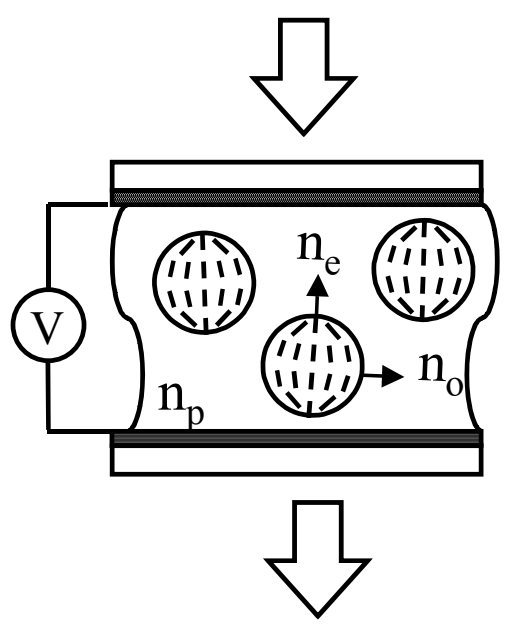

(b)

Figure 1.7: Schematic depiction of the polymer-dispersed liquid crystal material at (a) voltage-off state and (b) voltage-on state. 
For lens applications, the PDLC film with nano-sized LC droplets is desirable. Liquid crystal droplets are formed by phase separation of LC molecules from polymer. By increasing the speed of phase separation process, the nano-sized PDLC can be obtained. The nanoscale PDLC is optically isotropic with respect to the incident light and highly transparent in the voltage-off state. In a high-voltage state, the nano-sized droplets are reoriented by the electric field resulting in a phase change.

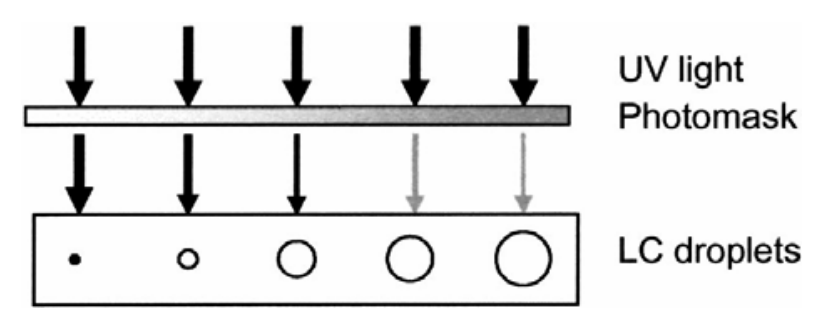

(a)

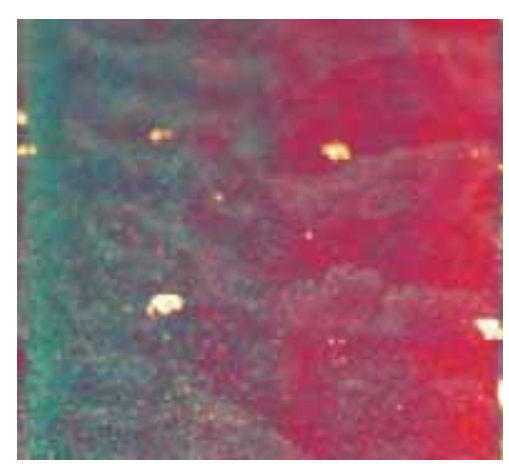

(b)

Figure 1.8: (a) Fabrication of an inhomogeneous PDLC using a patterned photomask. (b) Optical polarizing microscope photos of two PDLC samples with gradient droplet size distribution. LC composite: $70 \%$ NOA81 and $30 \% \mathrm{E} 7 . I=350 \mathrm{~mW} / \mathrm{cm}^{2}$. for $20 \mathrm{~s}$. 
In this dissertation, a novel PDLC device, which possesses inhomogeneous droplet size distribution, is demonstrated. The phase separation mechanism of the inhomogeneous PDLC is somewhat different from that of a uniform-sized PDLC. In the inhomogeneous PDLC, the gradient droplet size distribution is obtained by exposing the LC/monomer with a non-uniform ultraviolet (UV) light. A non-uniform UV intensity can be obtained using a patterned photomask, such as a continuously variable neutral transmission, as shown in Fig. 1.8(a). The photo-polymerization takes place preferentially in the high UV intensity regions. The consumption of monomers in these regions lowers their chemical potential. This pushes the monomers to diffuse from the low to high intensity region. On the contrary, the LC molecules diffuse form the high to low intensity direction to balance the chemical potential. As a result, in an inhomogeneous PDLC sample, not only droplet sizes but also their concentrations exhibit gradient distributions. Therefore, higher intensity would accelerate the phase separation and result in smaller droplet sizes, and vice versa. Figure 1.8(b) shows the microscopic photo of a PDLC film with nanoscale inhomogeneous droplet distribution. The LC droplets are too small to be resolved by microscope. It shows birefringence colors between crossed polarizers. ${ }^{21}$.

The region having smaller droplets also has a lower LC concentration. The average refractive index $\left(n=\left(n_{e}+2 n_{o}\right) / 3\right)$ of the LC droplets is larger than that of the polymer matrix $\left(n_{p} \sim n_{o}\right)$. The larger phase retardation observed in the larger droplet region. At $V=0$, a different phase retardation profile is indeed observed. The location with a larger droplet exhibits higher phase retardation. In the very high-voltage regime, the refractive index is decreased from $n$ to $n_{0}$ and the phase retardation becomes flat across the whole sample, regardless of droplet sizes. 
The PDLC driving voltage $V_{\mathrm{d}}$ (the critical voltage) is dependent on the droplet size $(D)$ as $^{22}$

$$
V_{d}=C / \sqrt{D},
$$

where $C$ is a constant. From Eq. (1.5), a larger droplet would have a lower operating voltage. Conversely, a smaller droplet would require a higher voltage. When a uniform voltage is applied, LC molecules in larger droplets will be reoriented first. An outstanding feature of our approach is the use of a uniform voltage to generate gradient phase profile. As a result, the electrode design is very simple.

The nanoscale LC droplets are randomly distributed, so that the devices are polarization independent and exhibit a fast response time. Due to the nanoscale droplet sizes, the operating voltage is higher than $100 \mathrm{~V}_{\mathrm{rms}}$.

\subsection{Polymer Network Liquid Crystal (PNLC)}

Unlike PDLC, the polymer network liquid crystal (PNLC) cell has the majority of LC molecules. Usually, the concentration of LC is higher than $90 \%$. In the low concentration regime $(1-2 \mathrm{wt} \%),{ }^{23,24}$ the polymer field improves the response time, however, the associated light scattering is quite strong in the visible region. As the polymer concentration increases to $\sim 3-8 \mathrm{wt} \%$, the polymer-stabilized LC (PSLC; also known as gel) is formed in a homogeneous or homeotropic cell. ${ }^{25,26}$. These PSLC cells exhibit an anisotropic light scattering behavior and has potential for reflective displays and telecom applications. ${ }^{27,28}$ 


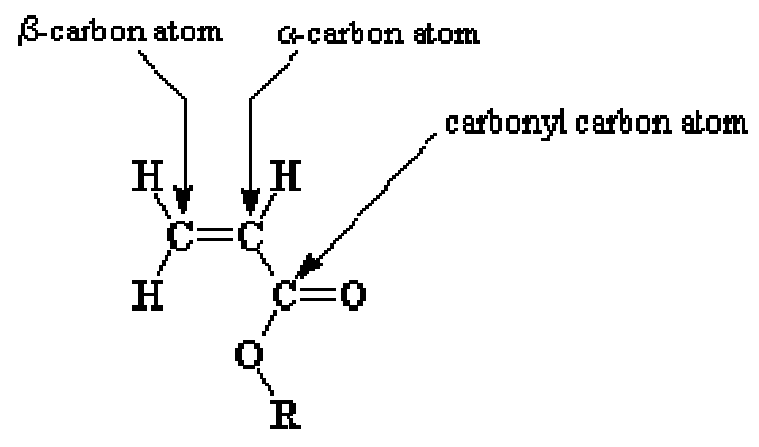

(a)

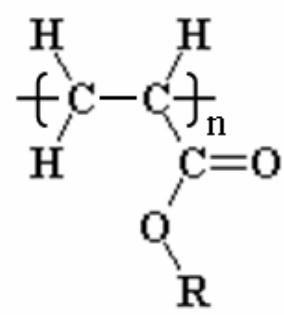

(b)

Figure 1.9: Chemical structures of (a) acrylate monomer and (b) acrylate polymer. R represents for any atom or group of atoms.

Polymer network liquid crystals are usually made from mixtures of liquid crystals and monomers. The monomer used usually is mesogenic with rigid core structures similar to those of liquid crystal molecules. ${ }^{29}$. The monomer consists of $1-5 \%$ photoinitiator. The monomer is usually an acrylate or a methacrylate because of their fast reaction rate. Acrylates are a family of polymers, which are a type of vinyl polymer. Acrylates are made from acrylate monomers. Acrylate monomers are esters which contain vinyl groups, that is, two carbon atoms double bonded to each other, directly attached to the carbonyl carbon, as shown in Figure 1.9(a). Figure 1.9(b) shows the acrylate polymer. The carbon-carbon double bond in a vinyl monomer, like ethylene, has a pair of electrons which is very easily attacked by the free radical, which is produced by photo-initiator. The unpaired electron, when it comes near the pair of electrons, cannot help but swipe one of them to pair with itself. This new pair of electrons forms a new chemical bond between the initiator fragment and one of the double bond carbons of the monomer molecule. The process is illustrated in Figure 1.10. Some acrylates have an 
extra methyl group attached to the alpha carbon, and these are called methacrylates, shown in Figure 1.11(a). One of the most common methacrylate polymers is poly(methyl methacrylate) (PMMA), shown in Figure 1.11(b). Monomer molecules must be at least bifunctional in the reaction. They must be able to react at two or more sites. If monomers with bifunctional groups are used, linear polymers can be formed.

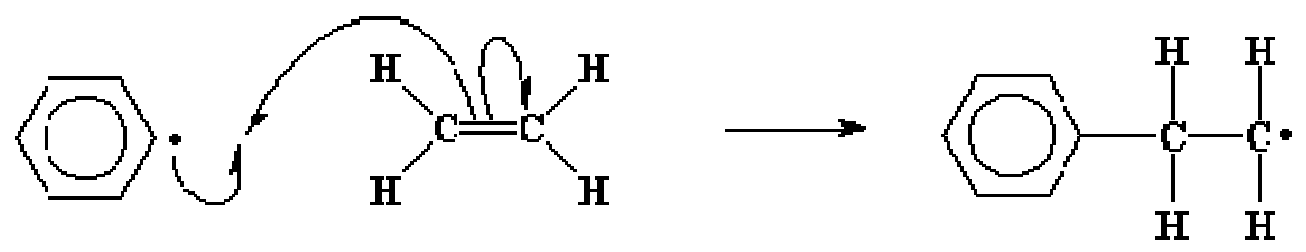

Figure 1.10: Illustration of free radical vinyl polymerization.

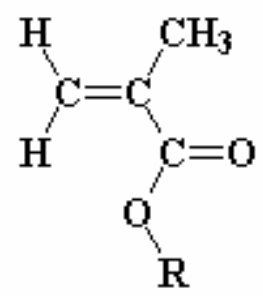

(a)

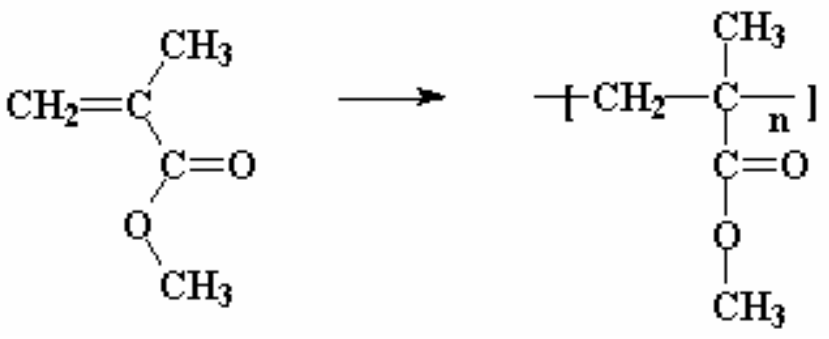

(b)

Figure 1.11: Chemical structures of (a) one kind of methacrylate monomer and (b) PMMA which is made by free radical vinyl polymerization from the monomer methyl methacrylate. 
The monomer can be directly dissolved in the liquid crystal. The photo-initiated polymerization is fast and used mostly to fabricate polymer network liquid crystals. Before polymerization, the mixture of the monomer and the liquid crystal is in a liquid crystal phase. The polymerization is anisotropic due to the aligning effect of the liquid crystal on the monomer and the anisotropic diffusion of the monomer in the liquid crystal. The anisotropic fiber-like polymer networks mimic the structure of the liquid crystal during the polymerization. ${ }^{30,31}$

When the LC/monomer mixture was injected in a homogeneous cell, the inner surfaces of the indium-tin-oxide glass substrates were overcoated with a thin polyimide layer and then rubbed in anti-parallel directions. The molecules of monomer and LC molecules are aligned by the buffed polyimide layers. After UV exposure, the monomer will be polymerized and become chain-like polymer network along the rubbing direction, as shown in Figure 1.12.

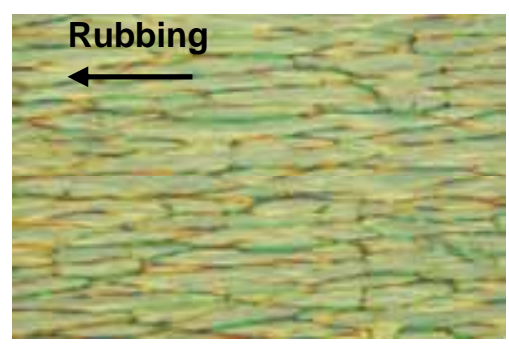

Figure 1.12: The microscopic photo of PNLC cell. The chain-like polymer networks are along the rubbing direction.

For prism and Fresnel lens applications, we also demonstrate an inhomogeneous polymer network concentration profile in the cell. The PNLC is produced by exposing the LC/monomer mixture with a uniform UV light through a patterned photomask. The 
higher UV exposure region results in a higher polymer concentration and smaller LC domains, so that the anchoring force of polymer in this region is higher. Its threshold voltage is higher. ${ }^{32}$. When the LC concentration exceeds $95 \%$; the operating voltage is low about $10 \mathrm{~V}_{\text {rms. }}$.

For fast-response LC modulator, we use a homogeneous PNLC with $\sim 10 \%$ polymer concentration.

\subsection{Phase-Separated Composite Film (PSCOF)}

Phase-separated composite film is a different phase-separation process, which is capable of producing a composite system consisting of a very thin layer of liquid crystal in contact with a similarly thin layer of polymer. The morphology of the polymer depends on the composition of the prepolymer-liquid crystal mixture and the rate of polymerization.

This phase-separated composite film (PSCOF) method can, in general, be used to prepare multilayer structures - for example, perpendicular to substrates to form an electrically controllable optical grating. In the simplest case, it yields adjacent uniform layers of the LC and polymer. The configuration of the optic axis in the LC layer can be controlled with an alignment layer on the substrate closest to the LC layer. The operation of a PSCOF device relies on changes in its birefringence in response to an applied electric field, as in conventional displays.

The technique used to construct the PSCOF structure (Figure 1.13) is essentially the same as that used for making polymer-dispersed liquid crystal (PDLC) devices. One starts with a pair of substrates coated with transparent indium-tin-oxide electrodes. One 
of the substrates was spincoated with a layer of a commonly used polymer, such as polyvinyl alcohol, and then rubbed to enforce LC alignment. The other substrate was left untreated. They were separated by the glass-bead spacers commonly used in the LC display industry. The PSCOF structure is designed to form between these two substrates such that the LC and the polymer layers are in contact with the rubbed and the untreated substrates, respectively. As a result, the LC layer is aligned by the rubbed substrate, while the polymer layer, which does not need to be aligned, is formed next to the untreated substrate. Phase separation is carried out by exposing the cell to UV light incident normally on the untreated substrate. At low levels of illumination a sufficient amount of diffusion can occur to allow the complete separation of LC and polymer.

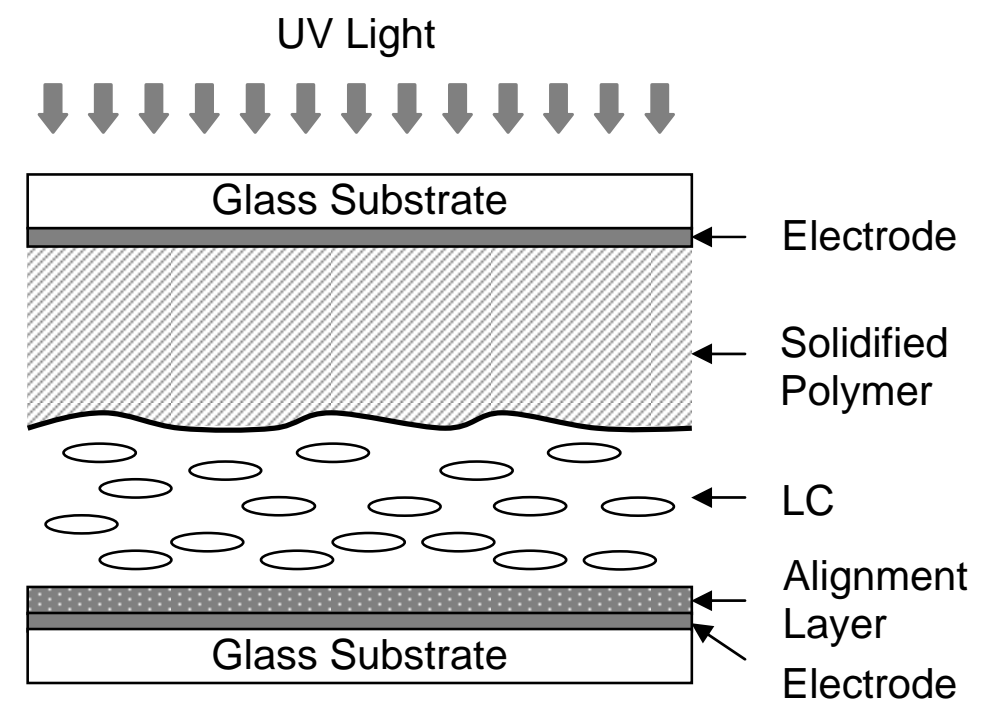

Figure 1.13: Schematic of the PSCOF structure. 


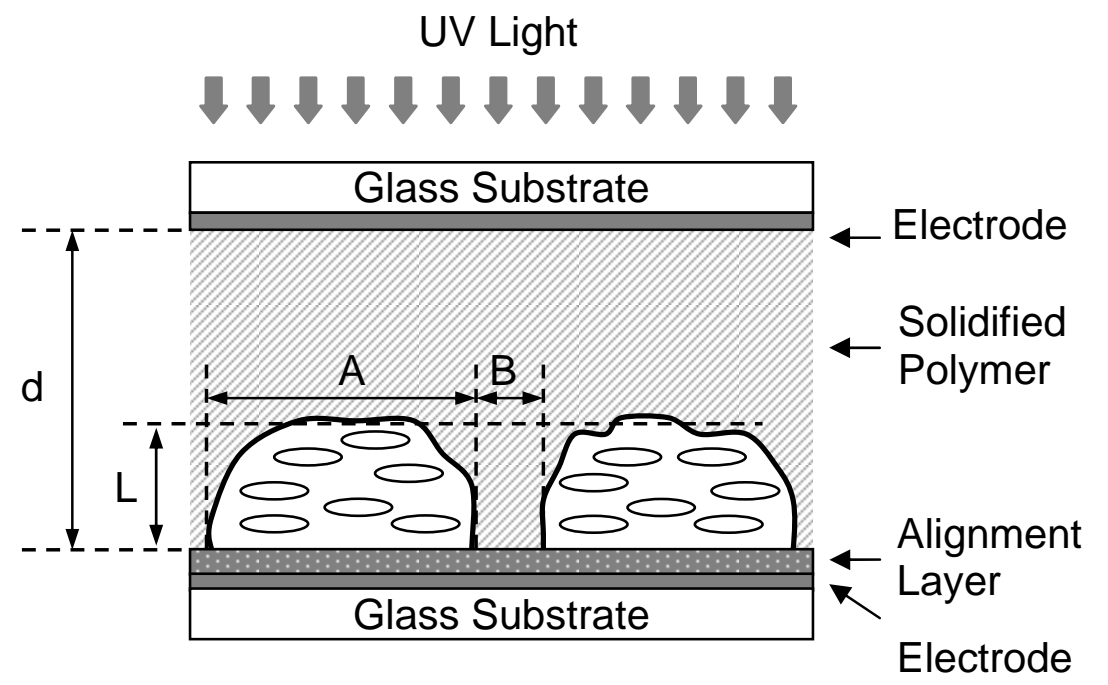

Figure 1.14: Schematic of the internal structure of a PSCOF device. $d$ is the cell thickness, which depends on the size of bead spacers used, and $L$ and $A$ are the average lateral and transverse dimensions of the LC volume. $B$ dimension characterizes the areas where the polymer-LC interface binds to the substrate on the opposite side.

The PSCOF can be made using similar materials to PDLC devices. ${ }^{33}$. A solution of the photocurable prepolymer NOA-65 and the LC, in an appropriate ratio, is introduced into the cell by capillary action at a temperature well above the isotropic-nematic phase transition. Phase separation results in a solidified film of polymer on the substrate close to the UV source and an LC film between the polymer film and the second substrate. Figure 1.14 shows another possible structure of PSCOF sample. The LC molecules are in homogeneous alignment resulting from the influence of the alignment layer on the adjacent substrate. The LC and polymer films are uniform except in regions where the polymer-LC interface bonds to the opposite substrate (Figure 1.15). These bonding sites are affected by the concentration and chemical nature of the LC compound, the alignment 
layer, the rate of phase separation, and the cell thickness $d$. The process can be optimized to reduce the average size and number of these bonding sites, resulting in an almost perfectly uniform LC film over the cell area (Figure 1.15(b)), which we refer to as the PSCOF structure. The formation of PSCOF is a joint effect of strong light absorption, slow polymerization, phase separation, and fast diffusion of small molecules.
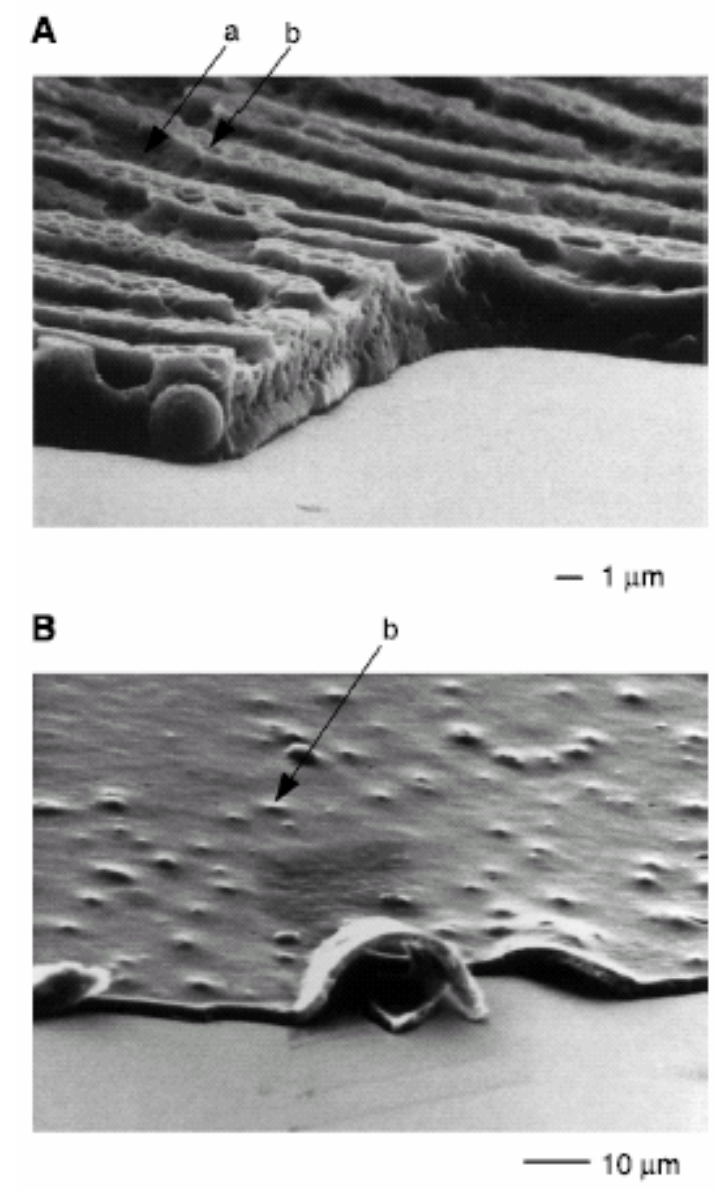

Figure 1.15: Scanning electron micrographs of the polymer film structure taken after removing the substrate close to the LC and washing away the $\mathrm{LC}$. The volume filled with LC (a) and the areas that were in contact with the opposite substrate (b) are indicated. (A) At $20 \%$ LC concentration, grooves or elongated LC droplets form along the rubbing direction after the phase separation. (B) The formation of nearly uniform parallel layers of LC and polymer (that is, a PSCOF structure) is confirmed for $40 \% \mathrm{LC}$ at the same rate of phase separation as in (A). At a faster rate of phase separation, PDLCs are formed. (Courtesy of Dr. S. Kumar of Kent State University) 
The PSCOF technology has advantages in the ease of fabrication, mechanical ruggedness, high resistivity, and flexibility of fine tuning the optical path length without requiring spacers of precise thickness, but most importantly, PSCOF devices prepared with ferroelectric and antiferroelectric LCs reveal technologically desirable characteristics including gray scale in ferroelectric (FLC) and antiferroelectric (AFLC) LC cells and a new switching mode in AFLC devices. In FLCs, the domain wall switching is suppressed, and the switching times at low fields are found to be two orders of magnitude faster than the conventional surface-stabilized FLC (SSFLC) devices. With the PSCOF technology, it now appears possible to build commercial electro-optical devices with ferroelectric and antiferroelectric LCs. 


\section{CHAPTER 2: PRISM GRATING USING POLYMER STABILIZED NEMATIC LIQUID CRYSTAL}

\section{$\underline{\text { 2.1 Introduction: Beam Steering and Blazed Grating }}$}

Laser beam steering is an important topic for free-space communications, military, optical interconnects, projection displays, and other general industrial applications. The goal is to deliver and control precisely the laser beams or other guided mode to a desired location. The most common technique is to reflect the light by mechanically controlled mirrors. Because of the nature of mechanical movement, the speed of the system is limited. Recently, researchers are seeking for nonmechanincal beam-steering devices with small and lightweight characteristics to replace the large mechanical systems. The other well-established beam steering device is the acousto-optic modulator, which has a severely limited angular range. Many new solid state/micro-component technologies such as optical MEMs, patterned liquid crystals, diffractive micro-optics and photonic crystals have been investigated to build small, ultra-light, rapidly steered laser beam sub-systems.

Liquid crystal materials are good candidates because of their low driving voltage and mature fabrication technology. Various liquid crystal (LC) beam steering devices have been developed for optical interconnects, optical communications, projection displays, and general industrial applications. ${ }^{1,3,5,8,11,12,34,35}$. Among these beam steering devices, the prism type is most promising. 


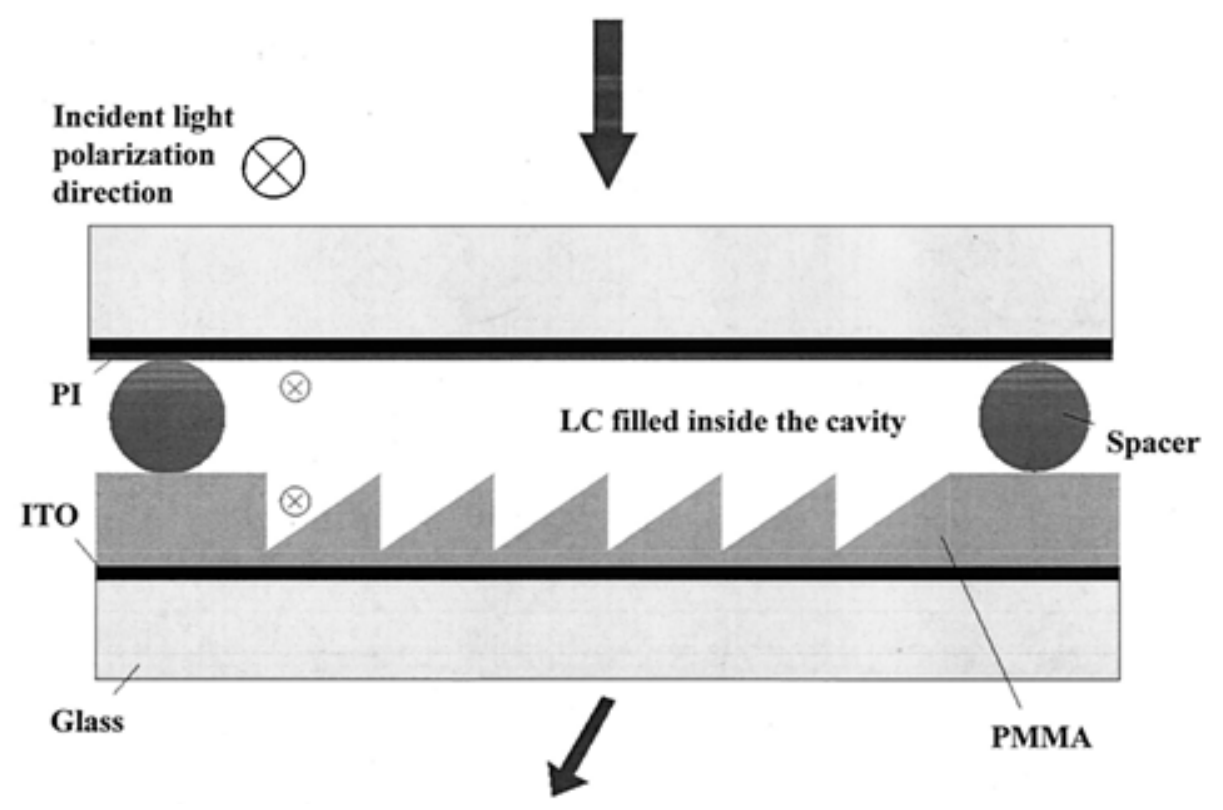

Figure 2.1: LC blazed-grating beam defector by using a glass substrate with a saw-tooth surface structure. PI: polyimide; ITO: indium-tin-oxide; PMMA: poly(methyl methacrylate).

A conventional simple grating structure produces several diffraction orders (first and higher orders). The theoretical diffraction efficiency of the first order beam is about $34 \%{ }^{36}$. The laser holographic blazing process shapes the grooves of the grating to concentrate the light into the $1^{\text {st }}$ order. The result is a much brighter spectrum. A welldesigned LC prism grating could reach $100 \%$ diffraction efficiency for the first order beam. ${ }^{12}$ One simple method to fabricate LC blazed grating is the use of a glass substrate with a saw-tooth surface structure, as shown in Figure 2.1. ${ }^{12}$. The thickness of LC layer in this structure is varying periodically and asymmetrically. The LC layer thickness in this structure is varying periodically and asymmetrically. The saw-tooth substrate is a poly(methyl methacrylate) (PMMA) blazed grating made by electron-beam (e-beam) lithography. The refractive index of PMMA is close to the ordinary index of the chosen 
LC and different from the extraordinary index. Therefore, in the voltage-off state, the inplane distribution of the optical path length of the incident light has a saw-tooth profile. The blazed grating would be switched off when the applied voltage is sufficiently high.

The angular locations of the possible diffraction orders can be expressed by ${ }^{37}$.

$$
m \lambda_{0}=L \sin \theta_{m},
$$

where $L$ is the width of the repeated unit, $\lambda_{0}$ is the grating's design wavelength, the integer $m$ is the diffraction order, and $\theta_{\mathrm{m}}$ is the angle at which each diffraction order appears. An additional requirement for a LC grating is that wavelets on either side of the junction between the repeated units must be in phase:

$$
m=\Delta n d / \lambda_{0}
$$

where $\Delta n$ is the birefringence of LC material and d is the cell gap. The integer $m$ in Eqs. (2.1) and (2.2) is identical.

An LC blazed grating can also be realized by use of the saw-tooth electric field with a uniform LC layer. Several approaches have been reported to achieve the saw-tooth electric field distribution, such as LC spatial light modulators, ${ }^{38}$. beam steerers with a tripe electrode, ${ }^{8,37}$ and a combination of low- and high-resistive electrodes. ${ }^{39}$.

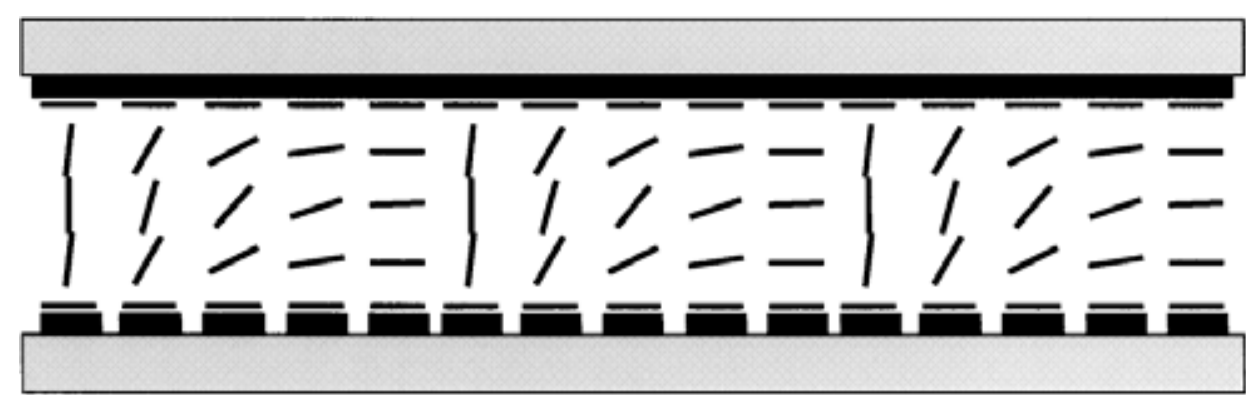

Figure 2.2: LC blazed grating by using an electric field with saw-tooth distribution. The liquid-crystal director varies only with $x$. 
Therefore, the LC-based prism grating is a strong contender for a high efficiency laser beam steering. However, the fabrication methods and operation mechanisms of those deflectors are rather complicated. ${ }^{3,5,8,12}$. There is an urgent need to develop new fabrication processes for LC prism grating.

In this work, we present a LC blazed grating using liquid crystal / polymer composite technique. A tunable LC blazed grating can be fabricated using a gradient refractive index nanoscale polymer dispersed liquid crystal (GRIN PDLC) device.. ${ }^{21}$ Owing to the nanoscale LC droplets involved, the GRIN PDLC lenses are highly transparent in the whole visible region, independent of polarization, and has $\sim 100 \mu \mathrm{s}$ response time. The shortcoming is that it requires a relatively high $\left(\sim 100 \mathrm{~V}_{\mathrm{rms}}\right)$ operation voltage. If the polymer network liquid crystal technique is used, the LC concentration is around $95 \%$ so that the operating voltage is relatively low. The PNLC is produced by exposing the LC/monomer mixture with a uniform UV light through a periodically varying density filter. The formed PNLC structure exhibits a periodically gradient polymer networks. The LC in the regions stabilized by a higher polymer concentration usually exhibits a higher threshold voltage..$^{32}$. Thus, when a homogeneous electric field is applied to the cell, the periodic gradient LC director distribution is generated and the prism effect occurs within each grating period. These microprisms help smoothen the grating structure and boost the diffraction efficiency.

\section{$\underline{\text { 2.2 Experimental Methods }}$}

Figure 2.3 illustrates the fabrication method of a PNLC prism grating. The key element is a patterned photomask. The photomask we used in this study is a periodically 
varying density pattern made by chromium with $300 \mu \mathrm{m}$ stripe width. When the uniform UV light (Loctite model 98016) passes through the photomask, the output intensity exhibits a saw-tooth profile within each period. The area with a stronger UV light would accelerate the polymerization process and aggregate a higher polymer concentration. Conversely, the area with a weaker UV exposure would have a lower polymer network concentration. As a result, a polymer network with periodic gradient morphology is formed. When a uniform electric field is applied, the LC directors are reoriented to different degrees. The region with a looser network packing has a lower threshold voltage than that of a denser region. Thus, the periodic gradient refractive index is formed within each grating period. As the applied voltage increases, the curvature of the gradient varies. In the high voltage regime, nearly all the LC molecules are aligned along the electric field direction. The gradient no longer exists and the grating effect vanishes.

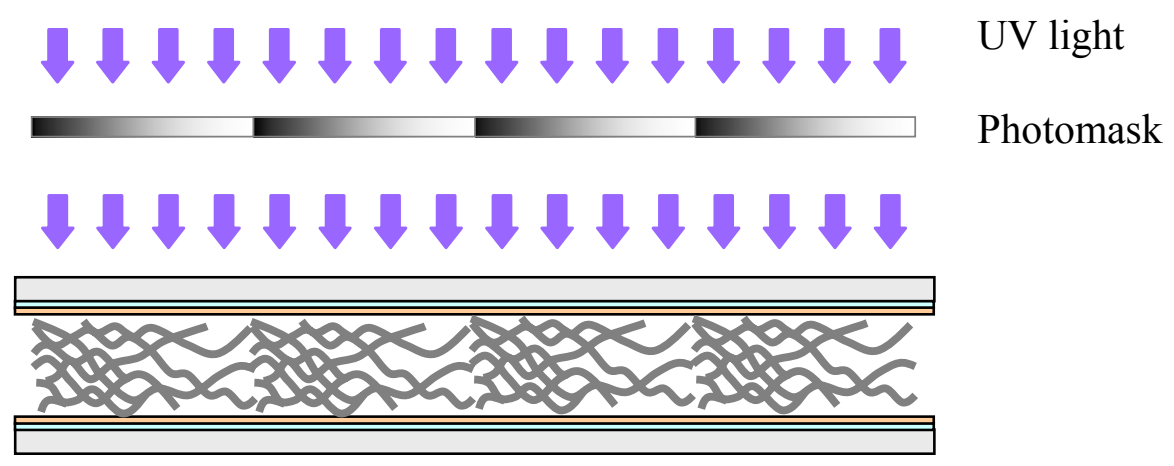

Figure 2.3: Method for fabrication PNLC prism grating. The patterned photomask has periodic spatially varying grating structures. 
In this experiment, nearly 3 wt $\%$ UV-curable monomer BAB-6 $(4,4[6-$ acyloyloxy)-hexyloxy]-1,1-biphenylene) was added to LC host (E48, Merck) along with a small amount of photoinitiator. The birefringence of E48 is $\Delta n=0.231$ at $\lambda=589 \mathrm{~nm}$ and room temperature. The BAB- 6 monomer has a rod-like structure with a reactive double bond at both sides. The LC/monomer mixture was injected to an empty homogeneous cell by capillary flow. The cell gap is $8 \mu \mathrm{m}$ and the thickness of the glass substrate is $1.1 \mathrm{~mm}$. The inner surfaces of the indium tin oxide glass substrates were overcoated with a thin polyimide layer and then rubbed in anti-parallel directions. Before polymerization, the LC and monomer molecules are aligned parallel with the rubbing direction. The curing UV intensity before the photomask is $12 \mathrm{~mW} / \mathrm{cm}^{2}$ and the exposure time is $60 \mathrm{~min}$.

\section{$\underline{\text { 2.3 Experimental Results and Discussion }}$}

In the voltage-off state, the prepared PNLC sample is highly transparent. A weak grating-like structure can still be observed by the naked eye. However, to resolve the detailed grating structures requires a polarizing optical microscope. On the microscope stage, the rubbing direction of the cell was oriented at $45^{\circ}$ with respect to the fast axis of a linear polarizer and the analyzer was crossed to the polarizer. Three photographs of the grating structures were taken at $V=0,1.5$, and $10 \mathrm{~V}_{\text {rms }}$. Results are shown in Figure 2.2. 

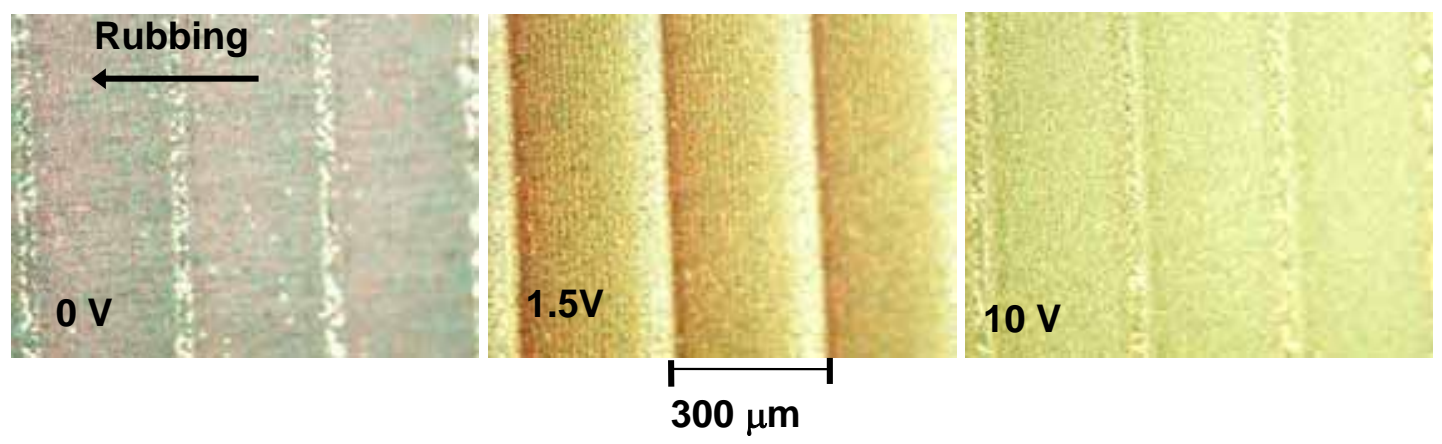

Figure 2.4: Microscope photographs of the PNLC gratings taken at $V=0,1.5$, and 10 $\mathrm{V}_{\mathrm{rms}}$. The LC used is E48 and cell gap $d=8 \mu \mathrm{m}$.

From Figure 2.4, the cell presents a weak grating-like structure at $V=0$. This is mainly due to the phase separation mechanism of the inhomogeneous PNLC, which is similar to that of the GRIN PDLC. ${ }^{21}$. The region having a looser polymer network has a higher LC concentration. As a result, the periodically inhomogeneous PNLC exhibits periodic gradient phase retardation within each grating period.

In our experiment, we found that as the applied voltage increases, the LC directors within each grating period start to be reoriented from the right side and gradually extend to the left. This implies that the right border in each grating period has a lower threshold voltage than that of the left. As depicted in Figure 2.3, the weaker UV exposure region leads to a lower polymer concentration which, in turn, results in a lower threshold voltage. This phenomenon also confirms that the gradient refractive index microprisms are indeed formed within each grating period. As the applied voltage reaches $1.5 \mathrm{~V}_{\text {rms }}$, the prism grating structure becomes clearer. However, at $10 \mathrm{~V}_{\mathrm{rms}}$ the prism grating structure vanishes. This is because the LC directors are reoriented along the electric field direction 
so that the phase gradient is erased. From Figure 2.4, the borders of the periodical stripes are not very sharp. This is attributed to the smooth edges of the photomask patterns.

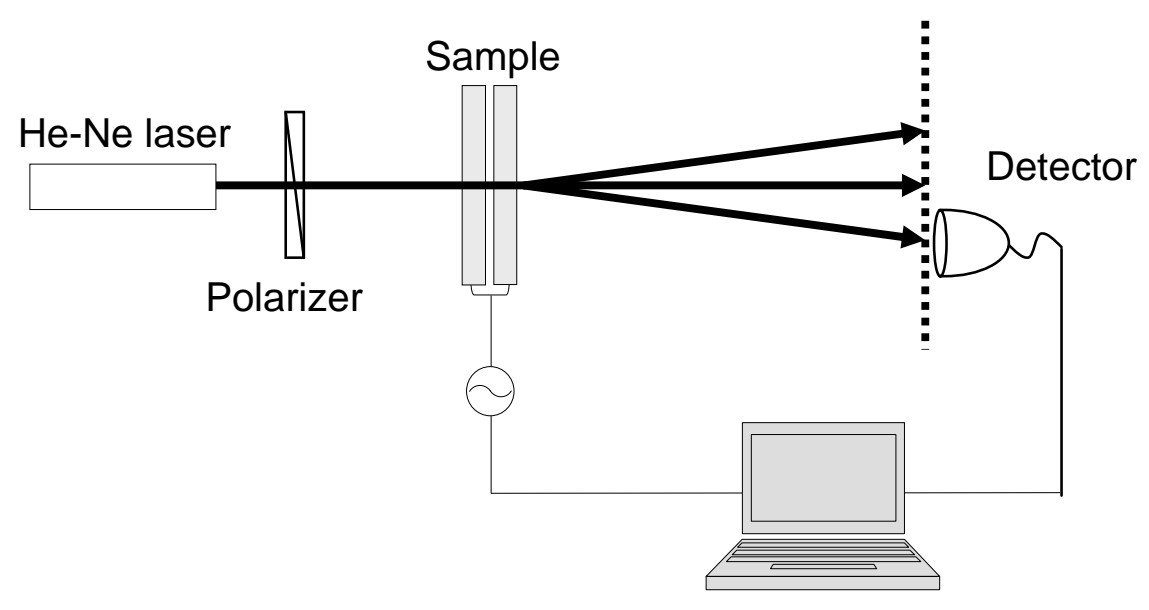

Figure 2.5: The experimental setup used for studying the diffraction patterns and measuring the electro-optical properties of the PNLC prism grating. $\lambda=543.5 \mathrm{~nm}$.

To quantitatively characterize the diffraction properties of the PNLC grating, we measured the transmitted laser power through a pinhole. An ac voltage of $1 \mathrm{kHz}$ sinusoidal waves was applied to the PNLC grating through a computer controlled LabVIEW system. Figure 2.7 plots the voltage dependent transmittance of the $0^{\text {th }}$ and first orders. We use the following expression to quantify the relative $0^{\text {th }}$, and the $1^{\text {st }}$. diffraction efficiency of the PNLC prism grating:

$$
\eta_{0,1}=T_{0,1} / T_{\text {total }}
$$

In Eq. (2.3), $T_{0,1}$ denotes the transmitted power of the $0^{\text {th }}$ and the first order beam, respectively, and $T_{\text {total }}$ is the total transmitted laser power including all orders. For the PNLC grating we fabricated, we found $\eta_{1}<20 \%$ at $V=0$. As the applied voltage 
increases, $\eta_{1}$ increases. At $V=1.5 \mathrm{~V}_{\mathrm{rms}}, \eta_{1}$. reaches $80 \%$ efficiency. As the voltage increases to $2 \mathrm{~V}_{\mathrm{rms}}, \eta_{0}$. is almost vanished, as shown in Figure 2.6. At this stage, higher order diffractions occur so that $\eta_{1}$ is actually lower than $80 \%$. When the operating voltage exceeds $2 V_{\text {rms }}$, the prism effect of the diffraction grating is gradually weakening and the prism grating behaves like a conventional LC grating. At a higher voltage ( $>3$ $\mathrm{V}_{\mathrm{rms}}$ ), light scattering takes place and the grating effect is degraded dramatically. The switching speed for the $0^{\text {th }}$. order was measured by using a square wave with $100 \mathrm{~ms}$ pulse width and $2 \mathrm{~V}$ amplitude. The turn-on time is $30 \mathrm{~ms}$ and turn-off time is $12 \mathrm{~ms}$.

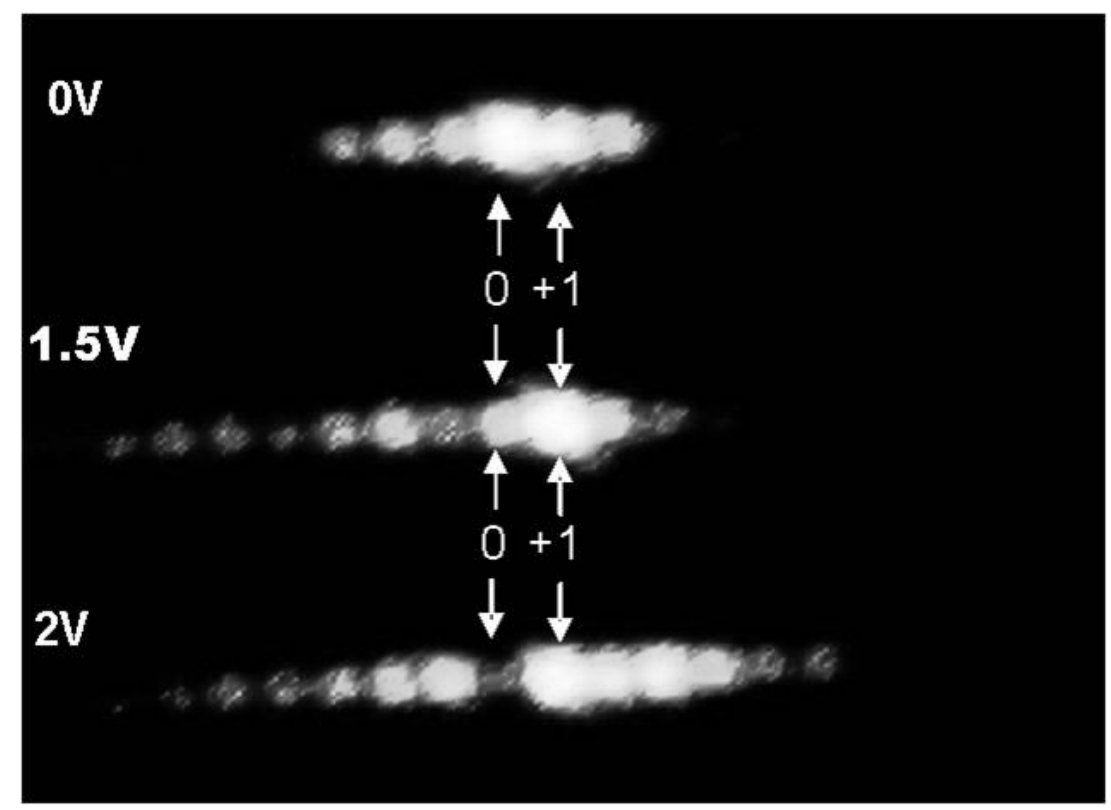

Figure 2.6: Photographs of the diffraction patterns observed in a dark room at $V=0,1.5$, and $2 \mathrm{~V}_{\mathrm{rms}}$. The PNLC prism grating has $300 \mu \mathrm{m}$ grating stripe.

Therefore, the PNLC prism grating can be operated in two regimes: $0-1.5 \mathrm{~V}_{\mathrm{rms}}$ for high first order diffraction efficiency, or $0-2 \mathrm{~V}_{\text {rms }}$ for switchable $0^{\text {th }}$ order diffraction. At $V=0$, the $20 \%$ noise of the first order beam should be eliminated if an ideal phase 
grating is generated through a sharp photomask pattern. Under such a circumstance, the higher order diffractions should be also suppressed and the first order diffraction efficiency is likely to be boosted to $100 \%$ at $V=1.5 \mathrm{~V}_{\text {rms }}$.

Our grating period is $300 \mu \mathrm{m}$ which is a little too wide so that the diffraction angle is small. To solve this problem, one should use a photomask with narrow periodic stripe width. In the mean time, the thickness of the substrate near the photomask side should be as thin as possible in order to avoid the diffraction from the photomask during UV light exposure. To sharpen the borders of the PNLC prism grating stripes, it is necessary to use a photomask with sharp joint borders between the stripes. Our results only serve the purpose for proving concepts.

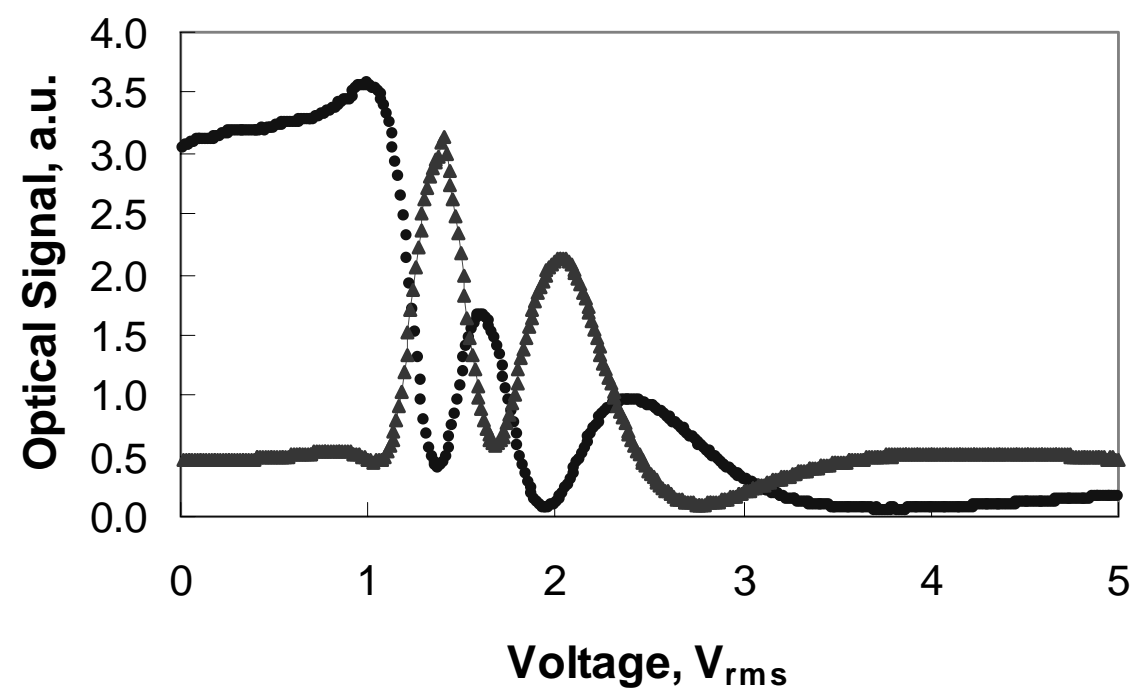

Figure 2.7: Voltage-dependent relative transmittance of the $0^{\text {th }}$. (circles) and the $1^{\text {st }}$ (triangles) order diffracted beams. The grating stripe width is $300 \mu \mathrm{m}$. 


\section{$\underline{2.4 \text { Conclusion }}$}

In conclusion, we have demonstrated an electrically switchable blazed grating using polymer network nematic liquid crystal. The diffraction characteristics are evaluated. The periodic gradient refractive index is realized by exposing the LC/monomer cell with UV light through a patterned photomask. Such a blazed grating exhibits not only a periodic grating structure but also a prism-like gradient refractive index with each grating period. The formed polymer network is an electrically switchable blazed grating. That means the microprisms are formed within each grating period when a uniform electric field is applied across the LC cell. The diffraction behavior is asymmetric. The first order exhibits high diffraction efficiency while the zeroth order has a high extinguishing ration. The optimized PNLC prism grating can be used as a beam deflector with high diffraction efficiency. 


\section{CHAPTER 3: SWITCHABLE FRESNEL LENS USING LIQUID CRYSTAL / POLYMER COMPOSITES}

\section{$\underline{\text { 3.1 Introduction: Fresnel Lens }}$}

An electrically tunable liquid crystal (LC) lens is a critical component for optical information processing, optical interconnection, beam deflector, and three-dimensional (3-D) display systems. Various LC lenses have been developed ${ }^{4,6,7,9,10,40-44}$, wherein the Fresnel zone plate (FZP) is very promising. In comparison with the refractive lenses, the Fresnel lens is simple to fabricate especially for the large aperture size.

Fresnel zone plates are very attractive devices due to their focusing abilities and compactness. ${ }^{45}$. The Fresnel zone plate was first used in stellar x-ray imaging, ${ }^{46}$. such as xray focusing and dispersing, imaging, and micro-beam forming. Later, FZP has been applied in the field of nuclear medicine. Recently, FZPs have been applied to optical

interconnection $^{2,47}$ and spatial light modulators, such as magneto-optic ${ }^{48}$ or ferroelectric liquid crystal devices. ${ }^{49}$. Fresnel lenses are also suitable for long distance optical communication, optical distance measurement, and space navigation.

In some applications, such as maskless lithography, ${ }^{50}$. projection displays, and variable optical attenuators using a zone plate modulator, it is highly desirable to continuously control the diffraction efficiency of the Fresnel lens. This tuning ability would eliminate the need for a spatial light modulator and mechanical light shutter. 
In a binary phase planar Fresnel lens, the phase difference between the adjacent zones can be tuned by the applied voltage. Maximum diffraction occurs when the phase difference is equal to an odd multiple of $\pi$ while no diffraction occurs for an even multiple of $\pi$. Through diffraction, the Fresnel zone plate works as a focusing element.

Many methods for fabricating FZP have been reported based on lithography,, ${ }^{51,52}$. etching techniques, ${ }^{53}$ thin-film deposition, ${ }^{54}$. and femtosecond laser machining. ${ }^{55}$. The phase shifts on the binary-phase Fresnel lenses remain fixed once they are fabricated. In some applications, a desirable feature of Fresnel lens is to control continuously the diffraction efficiency of the Fresnel lenses in order to eliminate the need for external amplitude spatial light modulators.

Electrically controllable Fresnel lens has been developed using liquid crystals in different approaches. A switchable binary-phase Fresnel lens can be fabricated using nematic liquid crystals. When the indium-tin-oxide (ITO) electrode is etched to form a zone plate $^{43,44}$ by electron beam lithography, only liquid crystals in odd zones or even zones can be driven by the electric field. The Fresnel lens is switched on when an external voltage is applied. This kind of Fresnel lens is sensitive to the light polarization. A polarization-independent liquid crystal Fresnel lens can be obtained by making the two neighboring zones with orthogonal LC directors. ${ }^{6}$. The relative phase between the two zones is controlled by a uniform electric field. A surface-relief structure is also introduced to fabricate the Fresnel lens. ${ }^{2}$ The surface of one substrate is created by reactive ion etching (RIE) to form Fresnel zones. The nematic liquid crystal is sandwiched between the surface-relief substrate and another flat substrate. The refractive index $n_{\mathrm{s}}$ of the surface-relief material is the same as the ordinary refractive index $n_{0}$ of 
liquid crystal material, i.e. $n_{\mathrm{s}}=n_{\mathrm{o}}$. The Fresnel lens is switched off when the liquid crystal molecules are reoriented perpendicular to the substrate, since the incident light encounters the same refractive index and sees no phase difference.

However, these fabrication methods are rather complicated. In this work, we report the fabrication of a Fresnel lens using liquid crystal/polymer composites. The key element for fabricating the Fresnel zone structure is a patterned photomask with circularly variable optical density in the radical direction.

Three different kind of thin LC/polymer films are used in this work: nanosized inhomogeneous PDLC, polymer network liquid crystal, and phase-separated composite film. The polymer formations are different depending on the LC concentration and the UV curing conditions. Polymerization induced phase separation method is used to fabricate the Fresnel lens. The different UV curing intensity due to the Fresnel zone patterned photomask creates the phase shift between odd and even zones. The Fresnel lens can be controlled by applying a uniform electric field.

\subsection{Theory of Binary Phase Fresnel Lenses}

For monochromatic radiation where the far-field approximation is valid, the

Fresnel zone plate performs generally as a lens with a series of foci of focal lengths $f_{1} /(2 j+1)$. The corresponding intensity of each focus is $1 /(2 j+1)^{2}$, where $j$ is an integer between minus and plus infinity, and $f_{1}$ represents the focal length of the principal focus of $j=0$. In most applications, only the principal focus with $j=0$ is required. The unwanted foci reduce the intensity of the image as well as the contrast of the image in the focal plane associated with the wanted focus, and create spurious images in other planes. 
These problems can be overcome by using multi-levels phase FZP. ${ }^{56,57}$. Some research work shows that the intensities of the high-order spots can be decreased by either increasing the number of Fresnel zones or by decreasing the size of the aperture. ${ }^{58}$. However, when the number of Fresnel zones is larger or the aperture of FZP is smaller, the far-field approximation is invalid because the spots of diffraction are within the nearfield region.

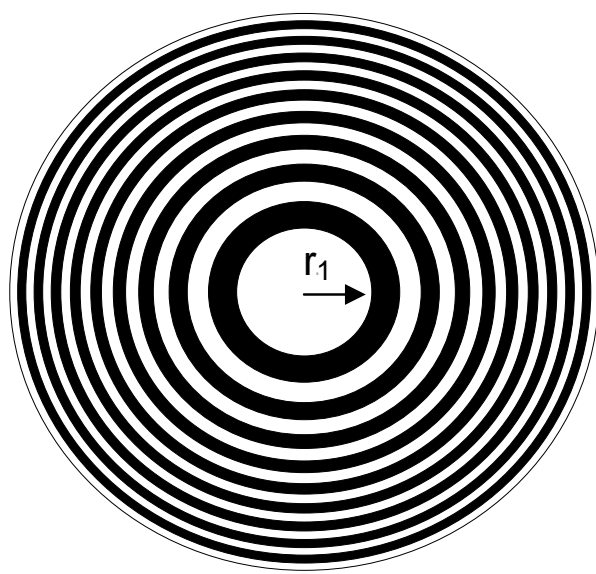

(a)

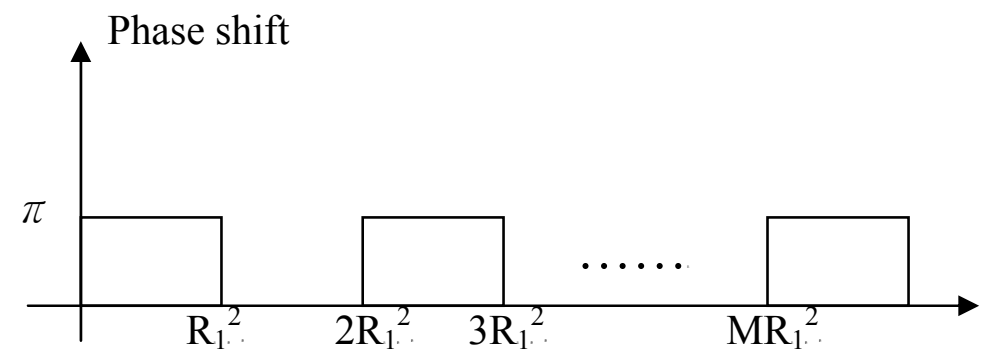

(b)

Figure 3.1: (a) Schematic diagram of a binary amplitude/phase Fresnel lens. In the amplitude lens every other zone is opaque, while in the phase lens they are all transparent with an induced $\pi$ phase shift between the neighboring zones. (b) The phase shift induced by a binary phase Fresnel lens as a function of $R^{2}$. The period of this function is $2 R_{1}{ }^{2}$. The amplitude of the transmittance function is unity. 
A typical binary amplitude Fresnel lens is shown in Figure 3.1(a), which consists of alternating opaque and transparent zones. All zones of a binary phase Fresnel lens (BP-FL), on the other hand, are transparent with an induced $\pi$ phase shift between neighboring ones. Because of its transparency, the throughput efficiency of a BP-FL is approximately four times better than a binary amplitude type. This makes BP-FLs more attractive for use in interconnection applications. Next we analyze the diffractive properties of a binary phase Fresnel lens. ${ }^{54,56}$

The radius of the $m^{\text {th }}$ zone $\left(R_{m}\right)$ in a Fresnel lens is given by

$$
R_{m}^{2}=m R_{1}^{2}
$$

where $R_{1}$ is the radius of the inner zone. The amplitude transmittance $t\left(R^{2}\right)$ is thus periodic in $R^{2}$, with period $2 R_{1}{ }^{2}$. When an FZP is illuminated by a plane monochromatic wave of wavelength $\lambda$, a multitude of diverging and converging spherical waves can be observed behind the FZP (Figure 3.2). The zeroth order is defined as the light passing through the FZP without being diffracted.

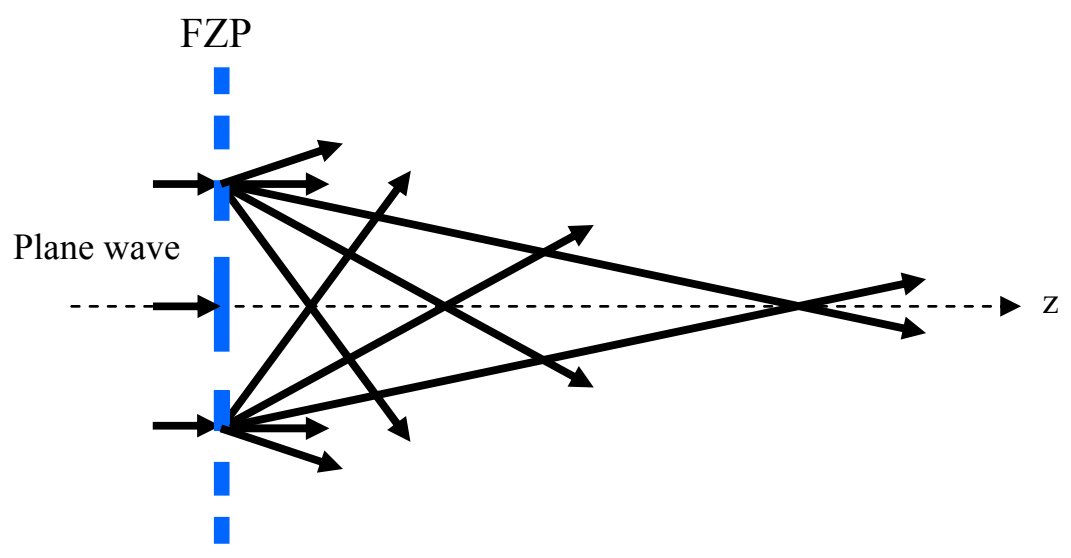

Figure 3.2: Diffraction of a plane wave at a Fresnel zone plate. 
By using scalar diffraction theory, we can derive equations for the $z$ coordinates of the focal planes and for the amplitudes of the diffraction orders. At discrete points along the axis of the lens, the optical field is at the maximum. The additional condition of a $\pi$ phase shift between neighboring zones (see Figure 3.1) eliminates some of these points.

The amplitude transmittance $t\left(R^{2}\right)$ can be expressed as a Fourier series expansion:

$$
t\left(R^{2}\right)=\sum_{n=-\infty}^{\infty} a_{n} \exp \left(i \pi n \frac{R^{2}}{R_{1}^{2}}\right)
$$

where $a_{n}$, the amplitude of the $n$th Fourier component, is

$$
a_{n}=\frac{1}{2 R_{1}^{2}} \int_{0}^{2 R_{1}^{2}} t\left(R^{2}\right) \exp \left(-i \pi n \frac{R^{2}}{R_{1}^{2}}\right) d\left(R^{2}\right) .
$$

For an incident plane wave with wavelength $\lambda$, the field distribution $U\left(x^{\prime}, y^{\prime}, z\right)$ at a point behind a BP-FL is determined from the Fresnel diffraction equation:

$$
U\left(x^{\prime}, y^{\prime}, z\right)=\iint t\left(R^{2}\right) \exp \left(\frac{i \pi}{\lambda z}\left[\left(x-x^{\prime}\right)^{2}+\left(y-y^{\prime}\right)^{2}\right]\right) d x d y,
$$

where the integration is over the aperture of the lens. In Eq. (3.4), an unimportant phase term outside of the integral has been omitted. Substituting Eq. (3.2) in Eq. (3.4) and using the relationship $R^{2}=x^{2}+y^{2}$, the field is found to be

$$
\begin{aligned}
U\left(x^{\prime}, y^{\prime}, z\right)= & \sum_{n=-\infty}^{\infty} a_{n} \exp \left(\frac{i \pi}{\lambda z}\left(x^{\prime 2}-y^{\prime 2}\right)\right) \iint \exp \left[i \pi\left(x^{2}+y^{2}\right)\right. \\
& \times\left(\frac{n}{R_{1}{ }^{2}}+\frac{1}{\lambda z}\right) \exp \left[\frac{-i \pi}{\lambda z}\left(x x^{\prime 2}-y y^{\prime 2}\right)\right] d x d y .
\end{aligned}
$$


In Eq. (3.5), the maxima occur when the exponent involving $\left(x^{2}+y^{2}\right)$ is equal to zero. These peaks are the foci generated by the FZP. For $z=z_{n}$ with

$$
z_{n}=-\frac{R_{1}^{2}}{n \lambda}, n=0, \pm 1, \pm 2, \pm 3 \ldots
$$

the field distribution, at the plane $z=z_{n}$, is therefore written by

$$
U\left(x^{\prime}, y^{\prime}, z\right)=a_{n} \exp \left(\frac{i \pi}{\lambda z}\left(x^{\prime 2}+y^{\prime 2}\right)\right) \operatorname{sinc}\left(\frac{x^{\prime} L_{x}}{\lambda z_{n}}, \frac{y^{\prime} L_{y}}{\lambda z_{n}}\right)+\sum_{q \neq n} b_{q} .
$$

In Eq. (3.7), the sinc function is the result of integrating over a rectangular aperture with dimensions $L_{x}$ and $L_{y}$; the second summation accounts for the contributions from fields that focus at other planes along the axis. From Eq. (3.7), it shows that the field maximum at plane $z_{n}$ is equal to $a_{n}$ (magnitude of the $\mathrm{n}^{\text {th }}$. Fourier component).

When the periodic $\pi$ phase shift is introduced for the additional condition, the amplitude transmittance for this case, as shown in Figure 3.1(b), can be represented by

$$
\begin{aligned}
t\left(R^{2}\right)= & \sum_{m=0}^{M / 2}\left[\exp (i \phi) \operatorname{rect}\left(\frac{R^{2}-2 m R_{1}^{2}-R_{1}^{2} / 2}{R_{1}^{2}}\right)\right. \\
& \left.+\operatorname{rect}\left(\frac{R^{2}-2 m R_{1}^{2}-3 R_{1}^{2} / 2}{R_{1}^{2}}\right)\right],
\end{aligned}
$$

where $M$ represents the number of the zones (we assumed it to be even), and $\phi$ is the induced phase shift, which is equal to $\pi$. The first rect function accounts for the phase shifted zones and the second for the zones with no phase shift. To simplify the analysis, we normalize the period, i.e. $2 R_{1 .}{ }^{2}=1$. By substituting Eq. (3.8) in Eq. (3.3), the amplitude of the $\mathrm{n}^{\text {th }}$. diffraction order can be obtained: 


$$
a_{n}=\frac{1}{2} \exp \left(\frac{-i \pi n}{2}\right)[-1+\exp (-i \pi n)] \operatorname{sinc} \frac{n}{2}
$$

Note that the integration was conducted over a period, i.e. $\mathrm{m}=0$. The bracket in Eq. (3.9) is either zero or -2 according to

$$
[-1+\exp (-i \pi n)]= \begin{cases}-2, & n= \pm(2 j+1) j=0,1,2, \ldots \\ 0, & \text { otherwise }\end{cases}
$$

Therefore, the maximum field amplitude is nonzero only for $n= \pm 1, \pm 3, \pm 5, \ldots$, and it is equal to $\operatorname{sinc}(n / 2)$. This result coupled with Eq. (3.6) yields the well-known result that such lenses act as both converging and diverging with foci located at

$$
f_{n}=-\frac{R_{1}^{2}}{n \lambda}, n= \pm 1, \pm 3, \pm 5 \ldots
$$

The maximum intensity, which is equal to the diffraction efficiency $\eta$, is then given by squaring the maximum field amplitude:

$$
\eta_{n}=I_{n}=\left|a_{n}\right|^{2}=\operatorname{sinc}^{2} \frac{n}{2}=\left(\frac{\sin \frac{n \pi}{2}}{\frac{n \pi}{2}}\right)^{2}, n= \pm 1, \pm 3, \pm 5 \ldots
$$

Diffraction efficiency into the primary focus $(n=-1)$ is determined from Eq. (3.12) to be $41 \%$. For secondary foci, the efficiencies decrease as $1 / n^{2}$.

Since the beam at the primary focus has the maximum intensity, it is suitable for both recording and reading holographic gratings. The beams from the secondary foci, however, can cause erasure of the primary gratings or record undesirable secondary gratings. In systems sensitive to this problem, multilevel phase Fresnel lenses which could suppress the higher order foci can be used. 
For multilevel phase components with discrete phase levels, a positive lens with a quantized phase profile, as shown in Figure 3.3, can be expressed mathematically as:

$$
t\left(R^{2}\right)=\sum_{k=0}^{2 M(N-1)}\left[\exp \left(\frac{2 i \pi k}{N}\right) \operatorname{rect}\left(\frac{R^{2}-2 k R_{1}^{2} / N-R_{1}^{2} / N}{2 R_{1}^{2} / N}\right)\right.
$$

where $L$ denotes the number of phase levels. In Eq. (3.13), a constant phase factor, $\exp [2 i \pi(N-1) / N]$, has been omitted to simplify the analysis. It is assumed that the phase steps are all of equal height which is $2 \pi / N$.

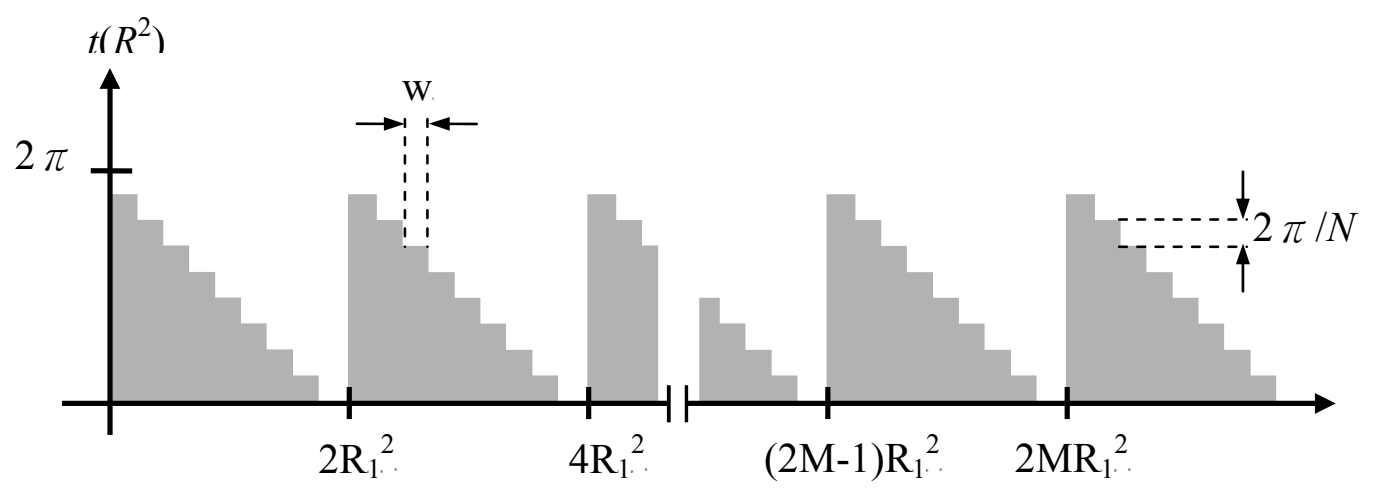

Figure 3.3: Phase profile of a diffractive lens with multiple discrete phase levels.

After we normalize the period, i.e. $2 R^{2}{ }^{2}=1$, the amplitude of the $n^{\text {th }}$ diffraction order can be obtained as:

$$
a_{n}=\exp \left(\frac{-i \pi n}{N}\right) \operatorname{sinc}\left(\frac{n}{N}\right) \frac{1}{N} \sum_{k=0}^{N-1} \exp \left[\left(-2 i \pi \frac{k(n+1)}{N}\right)\right]
$$

The sum of the right side of Eq. (3.14) is zero unless $n+1$ is a multiple of $N$. 


$$
\sum_{k=0}^{N-1} \exp \left[\left(-2 i \pi \frac{k(n+1)}{N}\right)\right]=\left\{\begin{array}{l}
L \text { if } n=j L-1, j \text { integer } \\
0 \text { else }
\end{array}\right.
$$

The diffraction efficiency $\eta$ of the $n=-1$ order is given as $\left|a_{-1}\right|^{2}$.

$$
\eta=\left|a_{-1}\right|^{2}=\operatorname{sinc}^{2} \frac{1}{N}=\left(\frac{\sin \frac{\pi}{N}}{\frac{\pi}{N}}\right)^{2} .
$$

Figure 3.4 shows the values of diffraction efficiency $\eta(L)$ graphically. The efficiency grows rapidly with $L$ and is as high as $95 \%$ for an eight-level lens. At the same time, by increasing $L$ the light intensity which is diffracted into other orders is reduced. This is visualized by Figure 3.5 which shows the spectrum of the foci of a diffractive lens as a function of the phase level $L$.

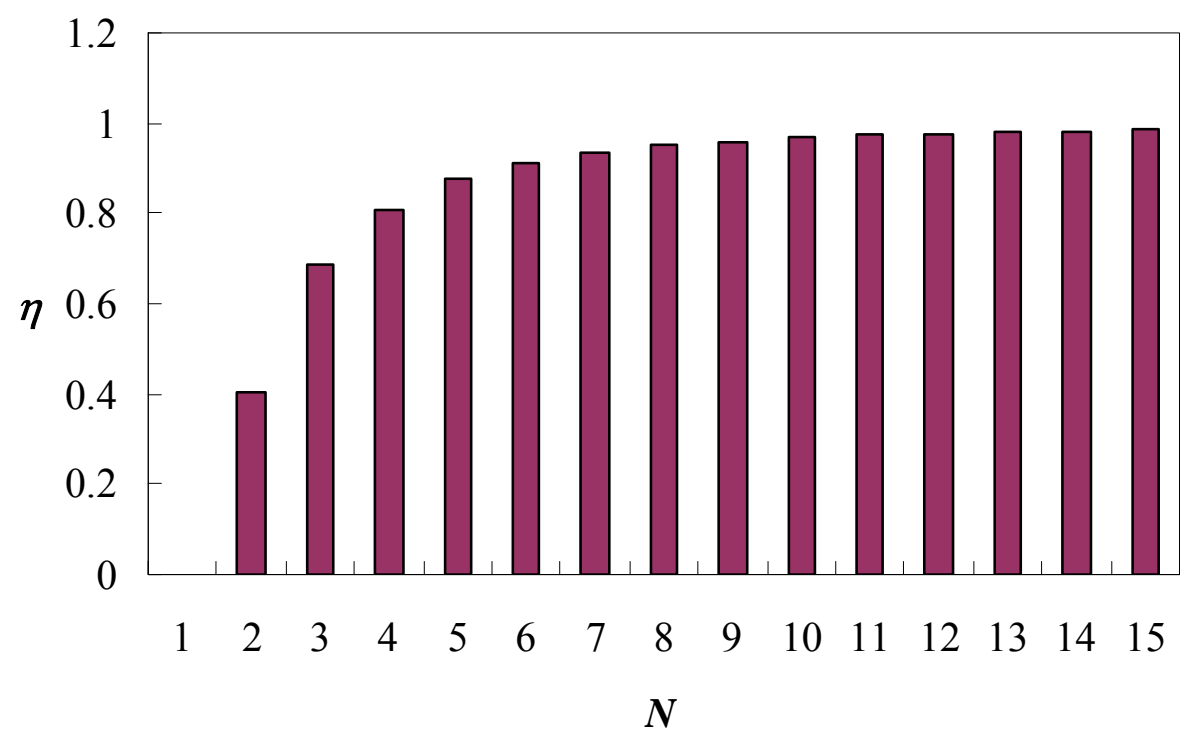

Figure 3.4: Efficiency of a diffractive lens as a function of the number of phase levels. 

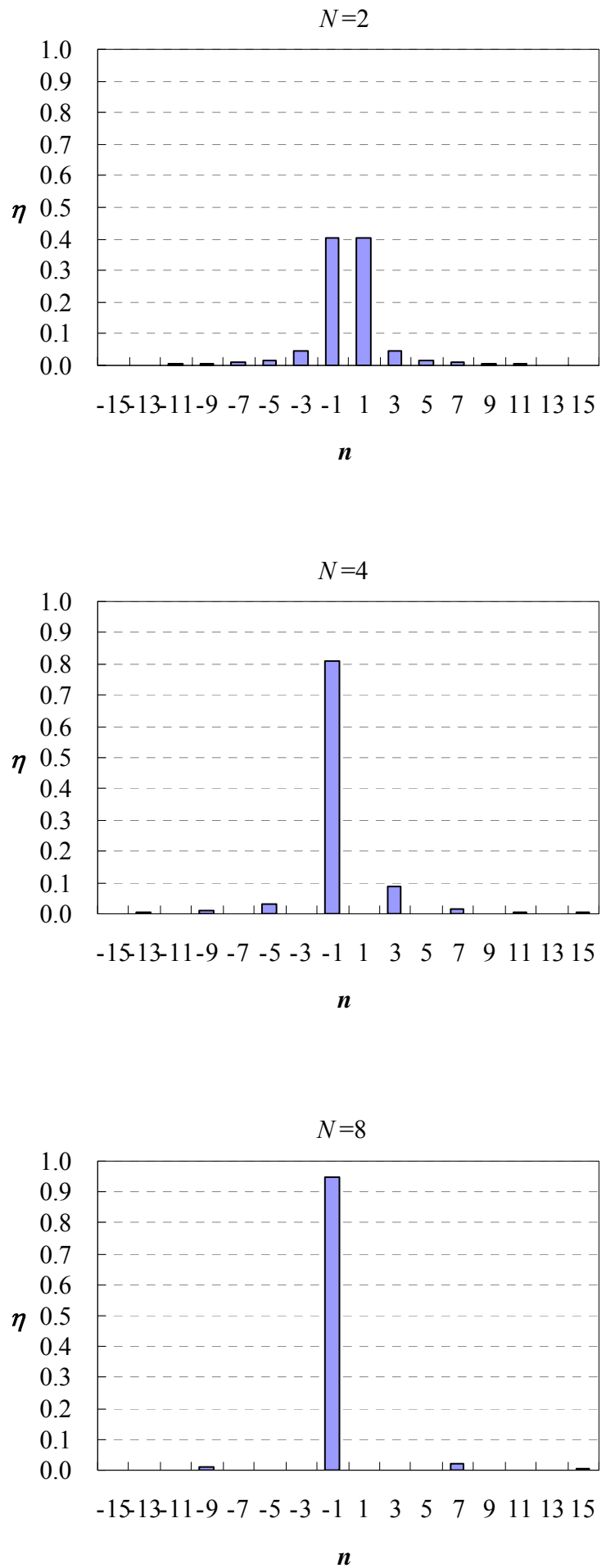

Figure 3.5: Focal spectrum of a diffractive lens as number $L$ of phase levels. 


\subsection{Fresnel Lens Using Nanoscale Polymer-Dispersed Liquid Crystal (PDLC)}

\subsubsection{Experimental Methods}

When the polymer concentration exceeds $\sim 30-40 \%$, the polymer-dispersed liquid crystal (PDLC) sample can be prepared. The LC molecules tend to be separated from polymer and aggregate to form LC droplets randomly distributed in the polymer matrix. In this work, to eliminate the light scattering due to the micro-sized LC droplets, higher monomer concentration is required to obtain the nano-sized PDLC. Therefore, the nanoscale PDLC films are transparent in the visible range.

Figure 3.6 illustrates the fabrication method of a Fresnel lens using nanoscale PDLC. The key element is the photomask (right) which has transparent odd zones and opaque even zones. Our photomask was produced by etching a chromium oxide layer using electron beam lithography. The radius $r_{1}$ of the innermost zone is $0.5 \mathrm{~mm}$ and the radius of the $n$th zone $\left(r_{n}\right)$ is given by $r_{n}^{2}=n \cdot r_{1}^{2}, n$ is the zone number. Our Fresnel zone plate consists of 80 zones in $1 \mathrm{~cm}$ aperture.

In our experiments, we mixed 26\% nematic E48 LC ( $\Delta n=0.231$ and $\Delta \varepsilon \sim 12.5)$ in an UV curable prepolymer NOA81 (Norland) host. Since NOA81 is sensitive to UV, the polymerization rate is relatively fast. The E48/NOA81 mixture was injected into a homogeneous LC cell composing of ITO-coated glass substrates. The cell gap is $d=15$ $\mu \mathrm{m}$. The bottom substrate surface is in proximity contact with the photomask. To avoid incomplete polymerization in the opaque zones, we illuminate the LC cell from both sides. A strong UV light $\left(I \sim 25 \mathrm{~mW} / \mathrm{cm}^{2}\right)$ illuminated from the bottom (photomask) and a 
weak UV light $\left(\mathrm{I} \sim 10 \mathrm{~mW} / \mathrm{cm}^{2}\right)$ from the top of the cell for $20 \mathrm{~s}$. Thus, the odd zones experience a higher UV intensity ( $\left(\sim 35 \mathrm{~mW} / \mathrm{cm}^{2}\right.$ ) than the even zones $\left(\mathrm{I} \sim 10 \mathrm{~mW} / \mathrm{cm}^{2}\right)$ so that their droplet sizes are smaller. In both zones, the PDLC droplets are formed after polymerization. However, unlike conventional PDLC, the LC droplet sizes are in subwavelength scale, since we control the polymerization rate. Thus, they do not scatter visible light.
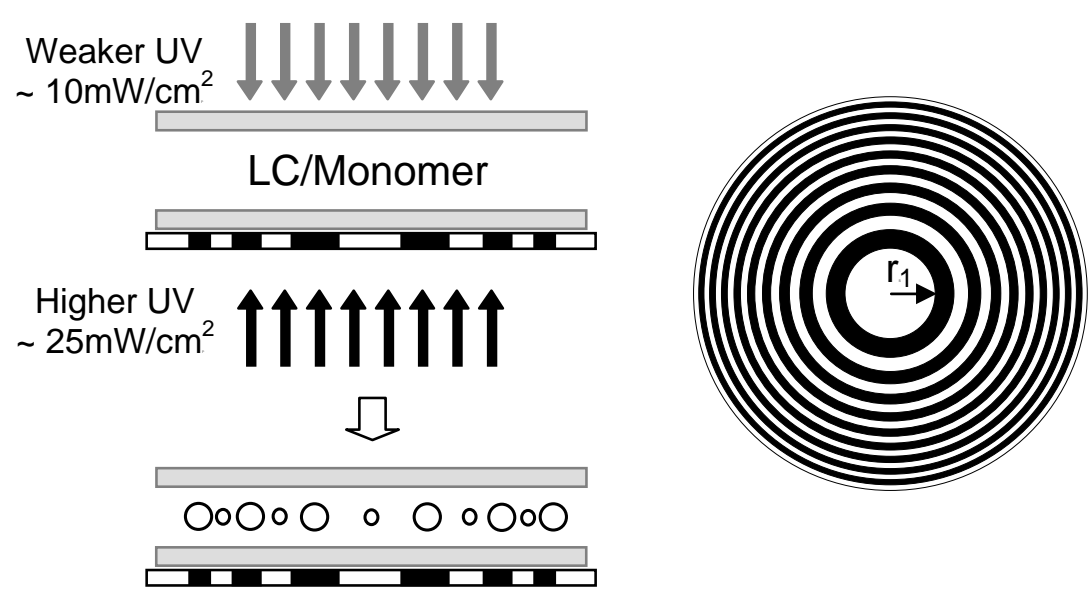

Figure 3.6: Left: method for fabricating nano-PDLC Fresnel lens. Right: the photomask showing Fresnel zone structure. The open circles indicate the inhomogeneous droplet size distribution.

\subsubsection{Operation Principle}

The LC/monomer in the zones cured with a higher UV intensity lead to smaller LC droplets. Conversely, the zones with a weaker UV exposure result in larger nanoscale droplets. When a uniform voltage is applied to the zone plate, the refractive index (or 
phase) of the zones with larger LC droplets are changed so that the beam diffraction efficiency and the focusing behaviors of the lens can be adjusted. Their electro-optic properties are independent of the incident light polarization, since the nanoscale LC droplets in the polymer matrix are randomly distributed. The lens exhibits a fast switching time due to the strong polymer anchoring force.

The odd zones of the prepared PDLC sample is highly transparent in the voltageoff state, while the even zones appear slightly bluish which implies the formed LC droplet is comparable to a blue wavelength. The Fresnel zone plate was inspected using a polarizing optical microscope where the sample was set between two crossed polarizers. At $V=0$, the birefringence colors of the Fresnel zones can be clearly observed (not shown). Such a Fresnel lens belongs to the binary-phase category. In a binary phase Fresnel lens, the focal length $\mathrm{f}$ is related to the innermost zone radius $r_{1}$ as $f=r_{1}^{2} / \lambda$, where $\lambda$ is the wavelength of the incident beam. The primary focal length of our lens was $\sim 50 \mathrm{~cm}$ (for $\lambda=633 \mathrm{~nm}$ ). Due to the higher-order Fourier components, Fresnel zone lens has multiple foci at $f, f / 3, f / 5$, etc. However, the majority of the incident light diffracts into the primary focus. The theoretical diffraction efficiency of the primary focus for the binary-phase Fresnel lens is $41 \%{ }^{59}$.

\subsubsection{Experimental Results and Discussion}

To characterize the light focusing properties of the PDLC Fresnel lens, we measured the image quality and the 3-D spot intensity profiles. Figure 3.7 depicts the experimental setup. The collimated He-Ne laser beam has $\sim 1 \mathrm{~cm}$ diameter which fills the 
entire zone plate. To study the light focusing properties of the primary focal point, the charge coupled device (CCD) camera (SBIG model ST-2000XM) was set at $50 \mathrm{~cm}$ from the Fresnel lens and the data were analyzed by a computer.

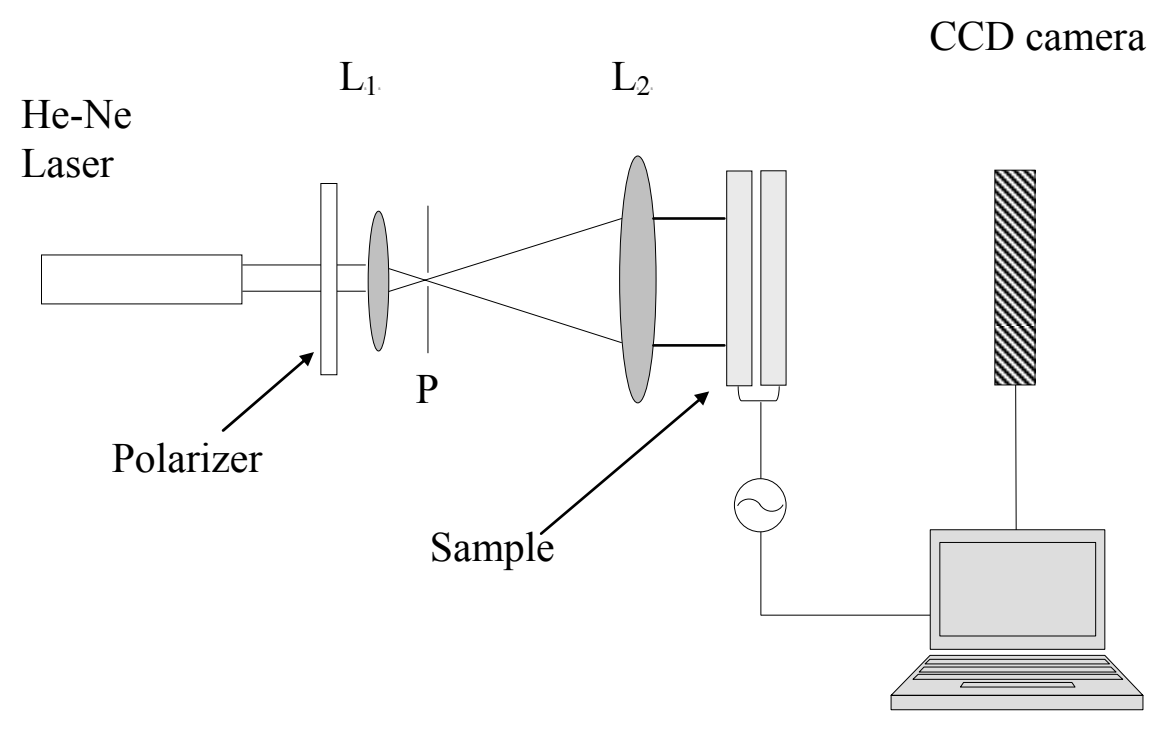

Figure 3.7: The experimental setup for studying the focusing properties of the PDLC Fresnel lens. $\mathrm{L}_{1}$ : focal length $50 \mathrm{~mm}, \mathrm{~L}_{2}$ : focal length $250 \mathrm{~mm}$, and P: $30 \mu \mathrm{m}$ pinhole.

To evaluate the image quality of the PDLC Fresnel lens, a black cardboard with transparent alphabet $\mathrm{R}$ was placed between lens $\mathrm{L}_{2}$ and sample. The $\mathrm{CCD}$ camera was set at $\sim 25 \mathrm{~cm}$ behind the sample. Figures 3.8(a) and 3.8(b) show the photos without and with the sample, respectively. When the sample is absent, no focusing effect occurs. Once the LC Fresnel lens is in position, a clear but smaller image is observed, although some circular noises exist due to diffraction. When the CCD camera was moved to the focal point, a tight spot appeared in the center, as shown in Figure 3.8(c). These results indicate that the sample indeed behaves like a lens. 


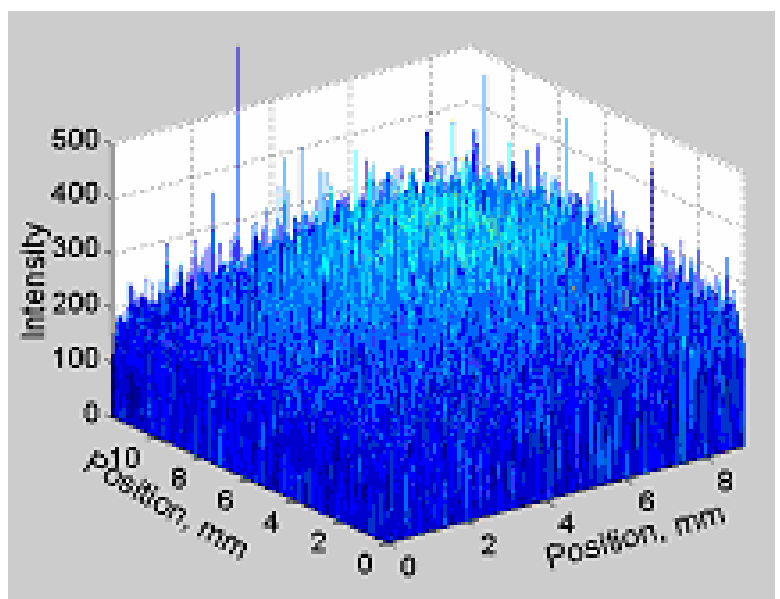

(a)

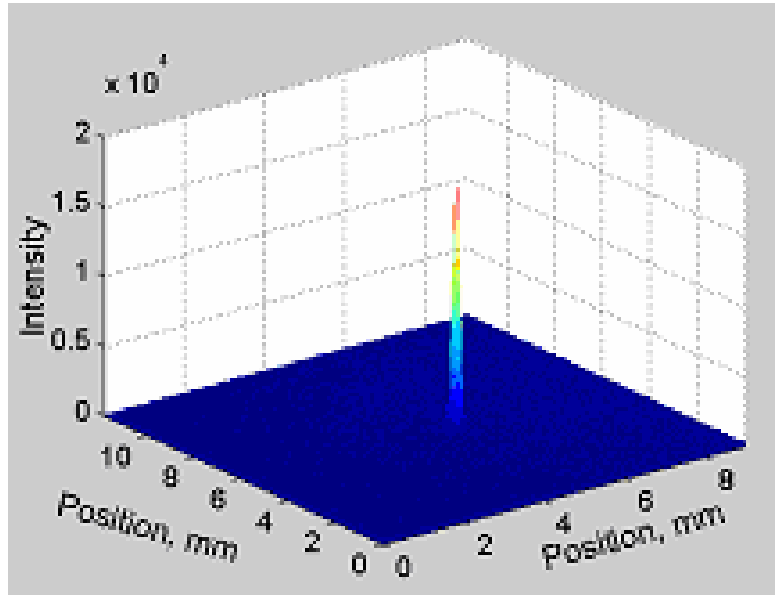

(b)

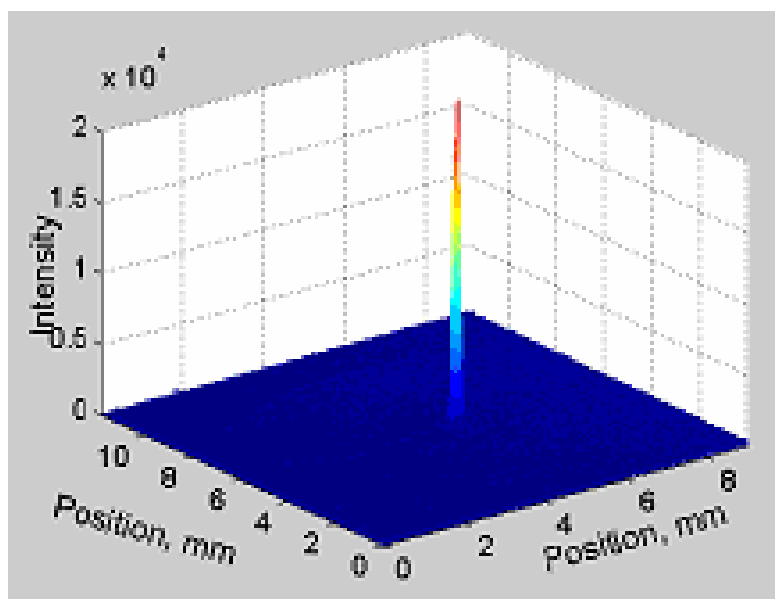

(c)

Figure 3.8: Spot intensity profiles measured by the CCD camera under different conditions. (a) Without sample, (b) with sample at $V=0$, and (c) $V=180 \mathrm{~V}_{\text {rms. The }}$ diameter of the Fresnel zone plate is $1 \mathrm{~cm}$ and the focal length is $50 \mathrm{~cm}$. 
To characterize the voltage-dependent light diffraction efficiency, we measured the 3-D profiles of the outgoing beams using a digital CCD camera. Results are shown in Figure 3.9. In the absence of the LC sample, the collimated light intensity is relatively uniform and the average intensity is 300 arbitrary units, as shown in Figure 3.9(a). When the nano- PDLC Fresnel zone plate is in position, a sharp focus occurs at $\sim 50 \mathrm{~cm}$. Figures 3.9(b) and 3.9(c) show the transmitted beam profiles at $V=0$ and $180 \mathrm{~V}_{\text {rms. }}$, respectively. The peak intensity reaches $\sim 10^{4}$. As the driving voltage increases, the peak intensity gradually increases. At $V=180 \mathrm{~V}_{\text {rms }}$, the peak intensity is nearly $1.5 \mathrm{X}$ as compared to that at $V=0$. Further increasing voltage would reorient the droplets along the electric field and then decrease the light intensity in the focal spot. To avoid the electrode breakdown, our maximum voltage is limited to $225 \mathrm{~V}_{\text {rms }}$.

The diffraction efficiency $(\eta)$ of the Fresnel zone plate is defined as

$$
\eta=\frac{p-p_{0}}{p_{t}} .
$$

In Eq. (3.17), $P$ denotes the transmitted laser power at the focal point, $P_{0}$ is the background noise, and $P_{t}$ is the total incident power after passing through the sample. In this experiment, we used a photodetector to measure the diffracted light power. Results are plotted in Figure 3.10. At $V=0$, the diffraction efficiency is $\eta \sim 32 \%$. As the voltage increases to $V=180 \mathrm{~V}_{\text {rms }}$ (or $12 \mathrm{~V}_{\text {rms }} / \mu \mathrm{m}$ ), the diffraction efficiency increases to $\sim 39 \%$, which is approaching the $41 \%$ theoretical limit. As the operating voltage exceeds 15 $\mathrm{V}_{\mathrm{rms}} / \mu \mathrm{m}, \eta$ starts to decline. 


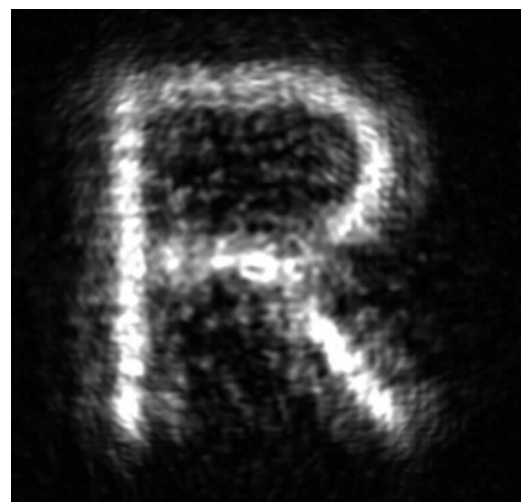

(a)

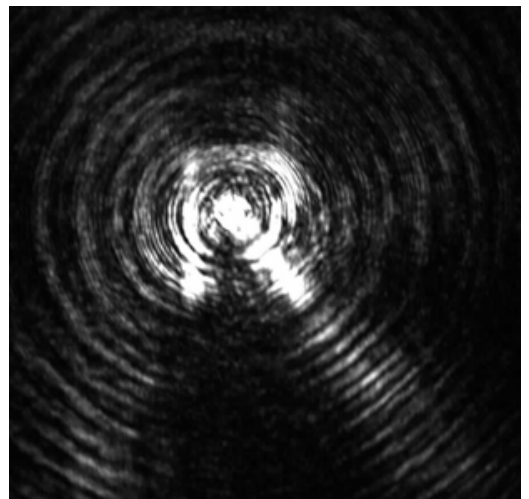

(b)

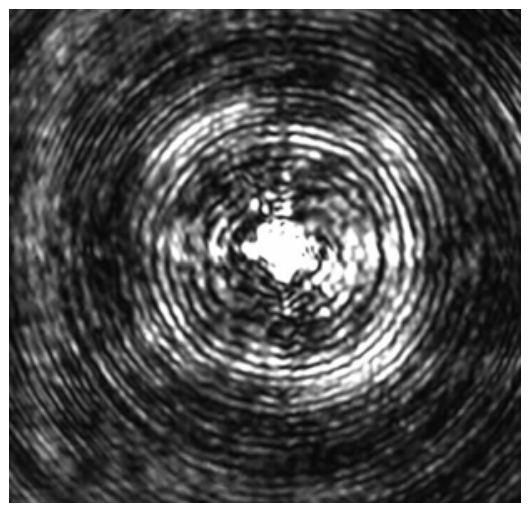

(c)

Figure 3.9: Imaging and focusing properties of the PDLC Fresnel lens recorded by a CCD camera. (a) without sample, (b) CCD at $25 \mathrm{~cm}$ from sample, and (c) CCD at focal point. 


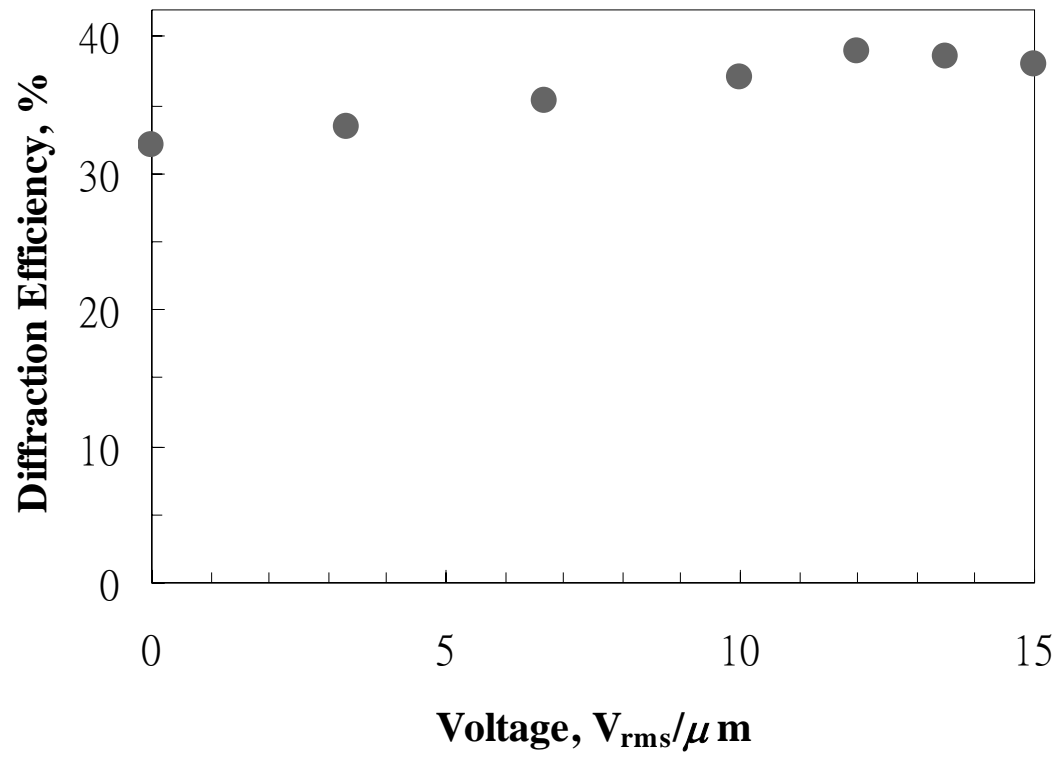

Figure 3.10: Voltage-dependent diffraction efficiency of the PDLC Fresnel lens. The LC employed is E48, cell gap $d=15 \mu \mathrm{m}$ and $\lambda=633 \mathrm{~nm}$.

The first order diffraction efficiency of a Fresnel lens is determined by the relative phase difference $(\Delta \delta)$ between the adjacent zones

$$
\Delta \delta=\frac{2 \cdot \pi \cdot d\left(n_{\text {odd }}-n_{\text {even }}\right)}{\lambda},
$$

where $d$ is the cell gap, and $n_{\text {even }}$ and $n_{\text {odd }}$ are the effective refractive index of the LC composite in even and odd zones, respectively. The maximum efficiency is reached when $\Delta \delta \sim \pi$. Our results plotted in Fig. 3.10 imply that the initial $\Delta \delta$ at $V=0$ is slightly larger than $\pi$. As the applied voltage exceeds $\sim 3 \mathrm{~V}_{\mathrm{rms}} / \mu \mathrm{m}$, the LC composites in the even rings start to be reoriented by the electric field while the odd rings remain basically unaffected due to their smaller droplet sizes. Thus, $\Delta \delta$ is approaching $\pi$ and diffraction efficiency slightly increases. As the voltage keeps on increasing, the LC composites in the even and 
odd rings are both reoriented by the electric field. Beyond $12 \mathrm{~V}_{\mathrm{rms}} / \mu \mathrm{m}$, both $n_{\text {even }}$ and $n_{\mathrm{odd}}$ are reduced and $\Delta \delta$ drops to below $\pi$. From this point on, the diffraction efficiency declines continuously.

The switching speed of the lens was measured using a square wave of $100 \mathrm{~ms}$ width and $180 \mathrm{~V}_{\text {rms }}(1 \mathrm{kHz})$ pulse amplitude. The rise and decay times were measured to be $200 \mu \mathrm{s}$. From this result, we estimate that the average LC droplet size in the even zones is $\sim 300 \mathrm{~nm}$. The odd zones should have a smaller droplet size due to the stronger UV exposure.

Similar to other composite systems, ${ }^{60}$ our subwavelength LC composites would also have imperfect spherical droplet shapes and nonuniform size distribution. The required voltage to reorient these fine droplets is relatively high. Holographic PDLC is such an example. ${ }^{11}$. Ideally, if the LC droplets can be completely reoriented by the electric field and the ordinary refractive index of the LC matches that of the polymer matrix, then $\Delta \delta \rightarrow 0$ and $\eta \rightarrow 0$ would occur in the high voltage regime. However, it is difficult to make $n_{\text {odd }}=n_{\text {even }}$ because the smaller LC droplets in the odd zones experience a stronger anchoring force than those in the even zones. Thus, the required voltage for completely turning off the Fresnel diffraction would be very high.

To overcome the high voltage problem of the subwavelength PDLC Fresnel lens, several approaches can be considered. For example, one can use a thinner cell filled with a high birefringence $(\Delta n>0.4)$ and high $\Delta \varepsilon$ LC. Adding a small amount of surfactant to the PDLC film is found useful for lowering the voltage while retaining a high diffraction efficiency. ${ }^{61}$ 


\subsubsection{Conclusion}

An electrically tunable Fresnel zone plate lens is demonstrated using nanoscale polymer-dispersed liquid crystal droplets. By using a photomask with Fresnel zone pattern during the polymerization process, the inhomogeneous droplet size distribution is obtained. In the voltage-off state, the diffraction efficiency of nanoscale PDLC Fresnel lens is $\sim 32 \%$. The measured diffraction efficiency is controllable by voltage. The maximum is close to the theoretical limit at $\sim 12 \mathrm{~V}_{\mathrm{rms}} / \mu \mathrm{m}$.

The image quality is studied. Relatively clear images are observed by using the nanoscale PDLC Fresnel lens. Due to the strong polymer anchoring force of the nanoscale droplets, this device exhibits fast switching speed $\sim 200 \mu \mathrm{s}$, although the required operating voltage is relatively high. The other advantages of such a Fresnel lens are simple fabrication, large aperture size, and polarization independent.

\subsection{Fresnel Lens Using Polymer Network Liquid Crystals (PNLC)}

\subsubsection{Experimental Methods}

In this work, we demonstrated a Fresnel lens using polymer network liquid crystal (PNLC). The fabrication procedure is relatively simple and the device can be operated below 10 volts with fast response time. Such a device works well for a linearly polarized light. This device has the potential for long focal length and large aperture size. 
The key element for fabricating the Fresnel zone plate is still a patterned photomask that was produced by etching a chromium oxide layer using electron beam lithography. Figure 3.11 depicts a simplified zone plate pattern and the corresponding LC molecular orientations in the voltage-off state. The innermost zone has radius $r_{1}=0.5 \mathrm{~mm}$ and the $n^{\text {th }}$. zone has radius $r_{n}$. which satisfies $r_{n}^{2}=n r_{1}^{2} ; n$ is the zone number. Our zone plate consists of 80 concentric rings within $1 \mathrm{~cm}$ diameter. During UV (Loctite model 98016) exposure, the photomask was in proximity contact with the LC cell. As shown in Figure 3.11, the odd zones are transparent and even zones are opaque. Thus, polymerization process would first take place in the odd zones resulting in a higher polymer concentration and higher threshold voltage $\left(V_{\text {th }}\right)$. Although even zones are opaque, monomers could still diffuse to these regions. Therefore, we further cured the cell with a weaker UV light after the photomask had been removed. The even zones have a lower polymer concentration so that their threshold voltage is lower. An LC polymer network replicating zone plate configuration is thus formed.

To fabricate LC zone plate, we mixed 5\% UV curable monomer bisphenol-Adimethacrylate in a LC host (Merck MLC-6252, $\Delta n=0.078)$. This monomer has a rodlike structure with a carbon-carbon double bond at both ends. The LC/monomer mixture was injected to a homogeneous cell composing of ITO (indium-tin-oxide)-coated glass substrates. The inner surfaces of the ITO-glass substrates were coated with thin polyimide layers and rubbed in anti-parallel directions. The pretilt angle is $\sim 3^{\circ}$, and cell gap is $d \sim 5 \mu \mathrm{m}$. During exposure, the cell was in proximity contact with the photomask. The UV light with intensity $I \sim 40 \mathrm{~mW} / \mathrm{cm}^{2}$. was used to cure the cell from the photomask 
side for 15 minutes. The photomask was then removed and exposure $\left(I \sim 20 \mathrm{~mW} / \mathrm{cm}^{2}\right)$ continued for another 15 minutes.

\subsubsection{Operation Principle}

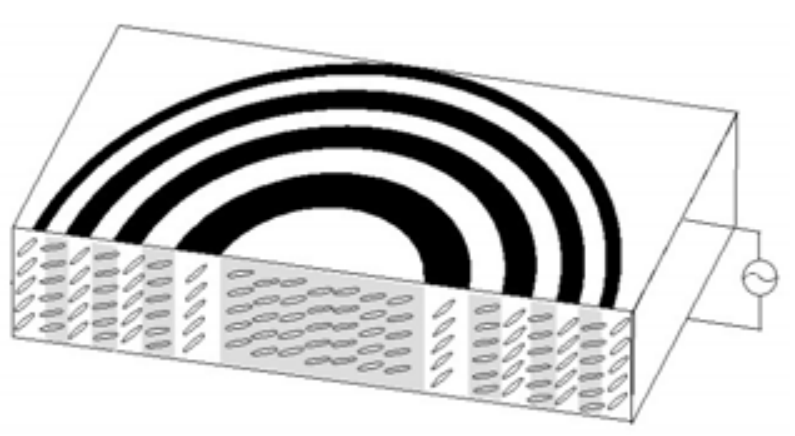

Figure 3.11: Schematic representation of the photomask patterns and the corresponding LC arrangement of the PNLC Fresnel lens.

The prepared polymer-stabilized LC sample is highly transparent in the voltageoff state. Figure 3.11 shows the schematic diagram illustrating the LC director arrangements of the polymer-stabilized LC Fresnel zone plate. In the low voltage regime, the LC molecules in the even zones are reoriented first while the molecules in the odd zones remain in the original alignment owing to their higher threshold voltage. A binaryphase Fresnel lens is thus formed. If the polarization of the incident light is parallel to the rubbing direction of the cell, the phase difference between the neighboring zones is $\phi=$ $2 \pi \delta n d / \lambda$, where $\delta n$ is the difference of the effective refractive indices in the adjacent zones, $d$ is the cell gap, and $\lambda$ is the wavelength of the incident beam. The induced phase 
shift is electrically tunable. The phase change corresponds to color change, when observed from an optical microscope through crossed polarizers. Under this visual inspection, the rubbing direction of the $\mathrm{LC}$ cell was oriented at $45^{\circ}$ with respect to the optical axis of the linear polarizer. The optical micrographs of the PNLC Fresnel lens were taken at different voltages. Results are shown in Figure 3.12.

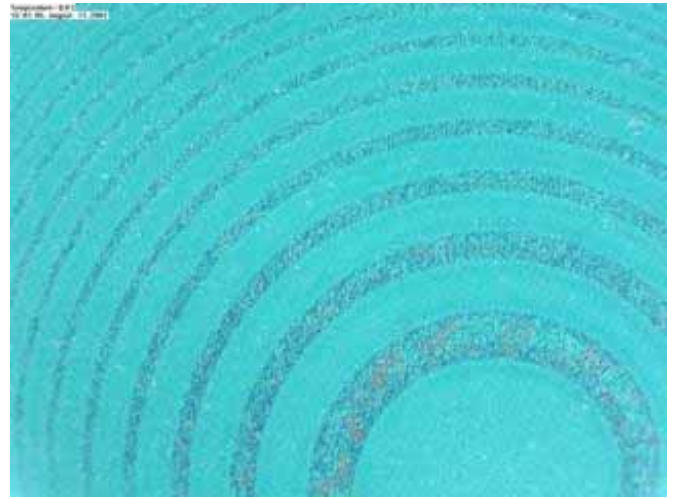

(a)

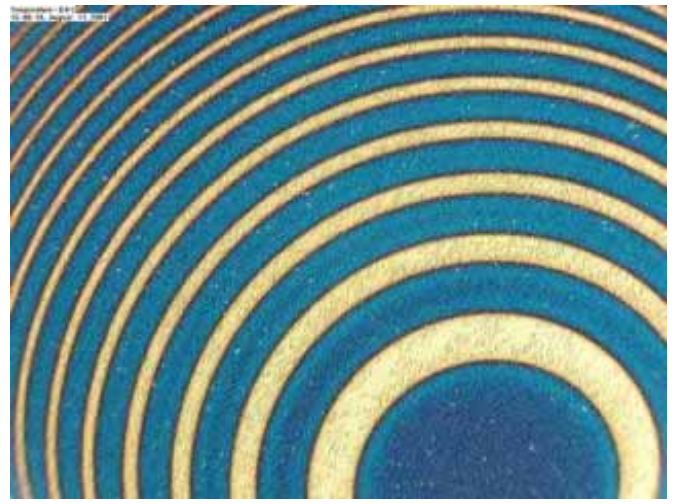

(c)

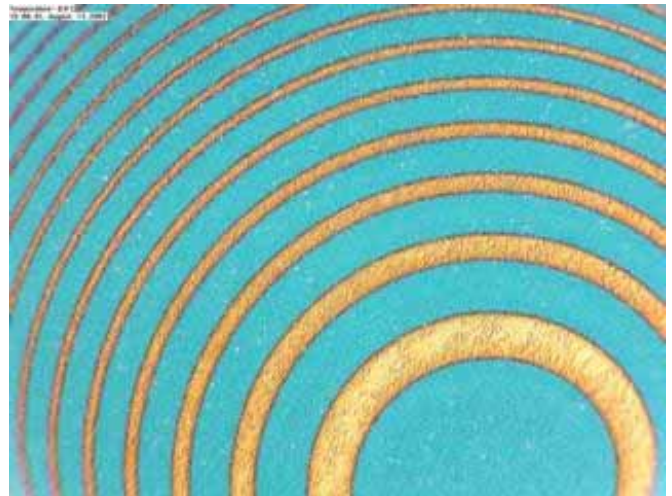

(b)

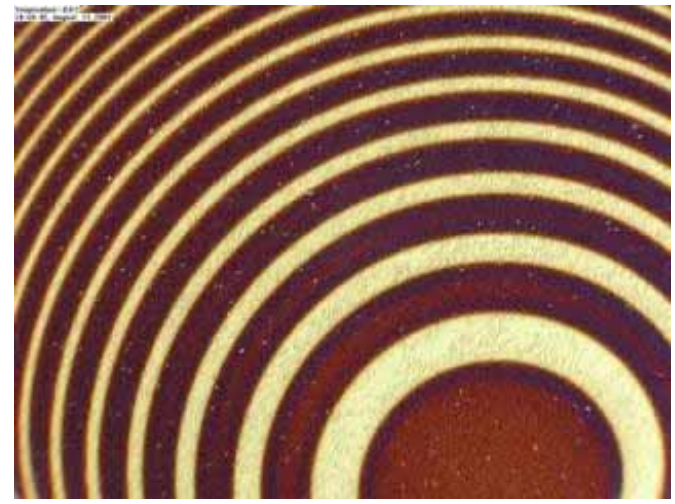

(d)

Figure 3.12: Microscope images of the PNLC cell at (a) $V=0$, (b) $4 \mathrm{~V}_{\mathrm{rms}}$, (c) $7 \mathrm{~V}_{\mathrm{rms}}$, and (d) $10 \mathrm{~V}_{\text {rms. }}$ The LC cell is sandwiched between crossed polarizers.

Figures 3.12(a-d) show a portion of the LC zone plate at $V=0,4,7$ and $10 \mathrm{~V}_{\mathrm{rms}}$, respectively. At $V=0$, the sample is optically homogeneous across the entire zone plate. 
As the voltage exceeds a threshold $\left(\sim 1.8 \mathrm{~V}_{\text {rms }}\right)$, the Fresnel zone starts to appear, as shown in Figures 3.12(b-d). The color change of the odd and even zones results from the refractive index change. In the higher voltage regime $\left(\sim 46 \mathrm{~V}_{\mathrm{rms}}\right)$, the bulk LC directors are orientated nearly perpendicular to the substrates, thus the zone structure is gradually erased. A drawback of the PNLC Fresnel lens is that it is polarization sensitive. For the light polarization perpendicular to the rubbing direction, it encounters the ordinary refractive index $n_{\mathrm{o}}$ of the liquid crystal layer. To overcome the polarization dependence, stacking two orthogonal homogeneous PNLC zone plates could be considered.

For a binary-phase Fresnel lens, the primary focal length $f_{11}$ is related to the innermost radius $\mathrm{r}_{1}$ as $f_{1}=r_{1}^{2} / \lambda$. The primary focal length of our LC Fresnel lens was estimated to be $50 \mathrm{~cm}$ for the He-Ne laser employed $(\lambda=632.8 \mathrm{~nm})$. Due to the higherorder Fourier components, Fresnel zone lens normally has multiple foci at $f, f / 3, f / 5, \ldots$, but most of light diffracts into the primary focus. The diffraction efficiency $\left(\eta_{n}\right)$ of these foci is $\eta_{n}=\operatorname{sinc}^{2}(\mathrm{n} / 2), \mathrm{n}= \pm 1, \pm 3, \pm 5, \ldots$ Therefore, the theoretical diffraction efficiency of the primary focus is $41 \%{ }^{62}$. However, the diffraction efficiency of our photomask was measured to be only $25.6 \%$, which is lower than the theoretical value of binary-phase structure because all the even zones are opaque.

\subsubsection{Experimental Results and Discussion}

For studying the focusing properties of the PNLC Fresnel zone plate, we use the same experimental setup shown in Figure 3.7. The output beam of the He-Ne laser was magnified by two convex lenses with the focal lengths $50 \mathrm{~mm}$ and $250 \mathrm{~mm}$. A pinhole 
with $30 \mu \mathrm{m}$ diameter was put at the focal point of the first lens serving as a spatial filter. The beam diameter was expanded to $\sim 1 \mathrm{~cm}$ just to cover the aperture of the zone patterns. A sheet polarizer was used with its optical axis parallel to the cell rubbing direction. Light focusing properties of the cell was measured using a CCD camera (SBIG Model ST-2000XM) connected to a computer. The CCD camera was set at a distance of $50 \mathrm{~cm}$ from the PNLC Fresnel lens.

Figure 3.13 shows the focusing property of the PNLC Fresnel lens displayed from the CCD camera. In the absence of sample, the laser beam has a Gaussian beam profile shown in Figure 3.13(a). When the sample is present and $V=0$, the PNLC lens possesses a small focusing effect, as shown in Figure 3.13(b). This is due to the different polymer concentration between odd and even zones which makes the effective refractive indices slightly different between the adjacent zones. At $V=8 \mathrm{~V}_{\mathrm{rms}}$, the focusing effect is intensified, as shown in Figure 3.13(c).

Figure 3.14 shows the image quality of the PNLC Fresnel lens using a transparent alphabet M placed between lens $\mathrm{L}_{2}$ and sample. The CCD camera was located at $\sim 27 \mathrm{~cm}$ from the sample. At $V=0$, an image of "M" with the original size was recorded by $\mathrm{CCD}$, as shown in Figure 3.14(a). When $8 \mathrm{~V}_{\text {rms }}$.was applied to the lens, the image "M" was obviously shrunken, as shown in Figure 3.14(b).

Figure 3.15 shows a representative example of the measured three-dimensional intensity distribution at the primary focal point of the LC Fresnel lens. At $8 \mathrm{~V}_{\mathrm{rms}}$, a sharp focal spot was observed. Figure 3.16 plots the measured laser intensity profiles at different voltages. In the voltage-off state, a slight focusing effect exists. At $V=8 \mathrm{~V}_{\text {rms, }}$, 
the peak intensity occurs. As the voltage continues to increase (say, $V=20 \mathrm{~V}_{\mathrm{rms}}$ ), the peak intensity decreases.

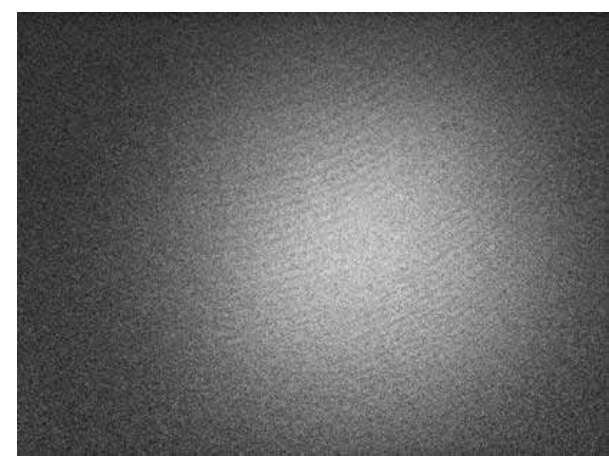

(a)

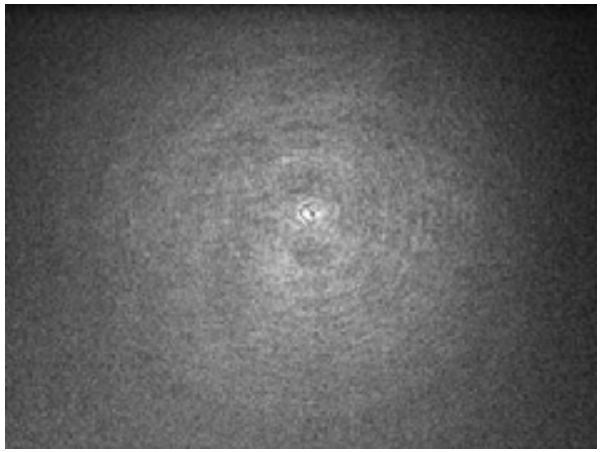

(b)

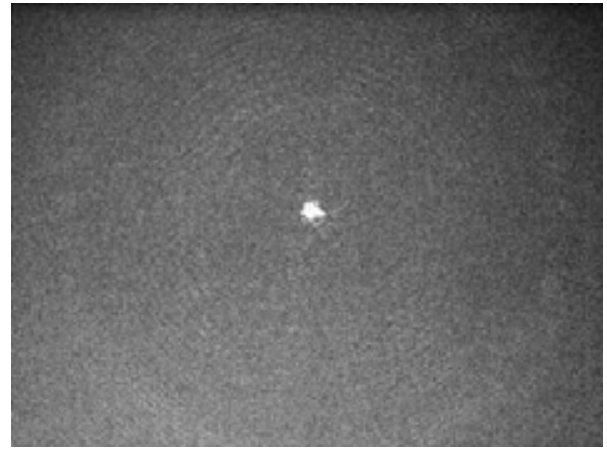

(c)

Figure 3.13: The observed laser beam images: (a) without LC sample, (b) with sample at $V=0$, and (c) with LC sample at $8 \mathrm{~V}_{\mathrm{rms}} \cdot \lambda=633 \mathrm{~nm}$. 


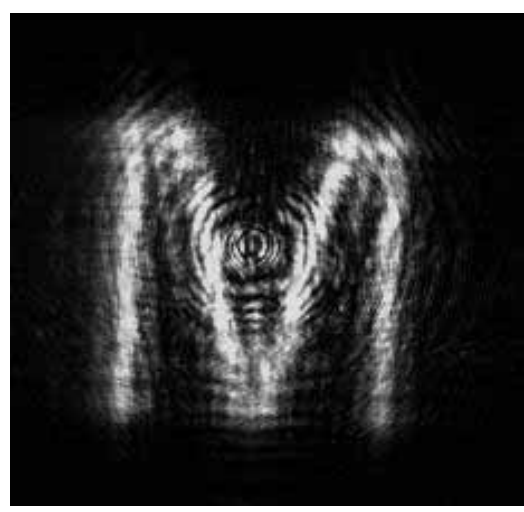

(a)

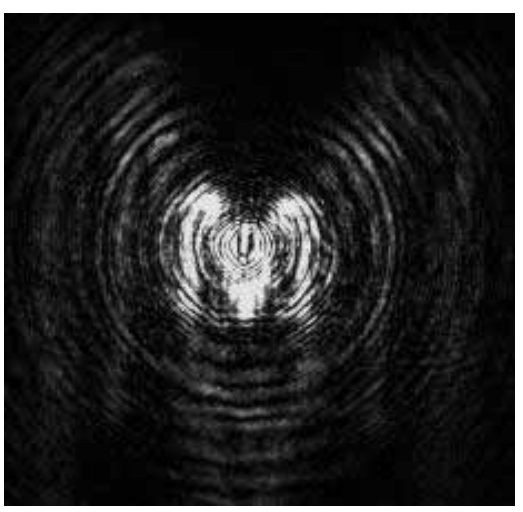

(b)

Figure 3.14: The observed laser beam images (a) at $V=0$ and (b) at $8 \mathrm{~V}_{\text {rms. }} \lambda=633 \mathrm{~nm}$. The CCD camera was $27 \mathrm{~cm}$ away from the sample.

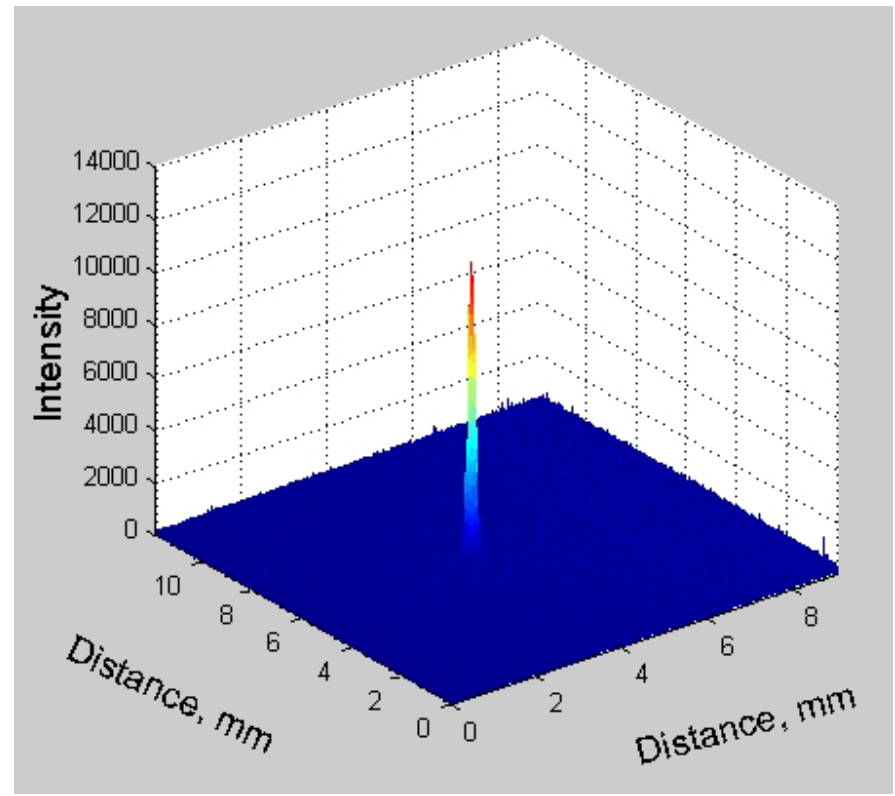

Figure 3.15: Beam intensity profile measured by the CCD camera at $V=8 \mathrm{~V}_{\text {rms. }}$ The diameter of the Fresnel zone plate is $1 \mathrm{~cm}$ and the focal length is $50 \mathrm{~cm}$. 


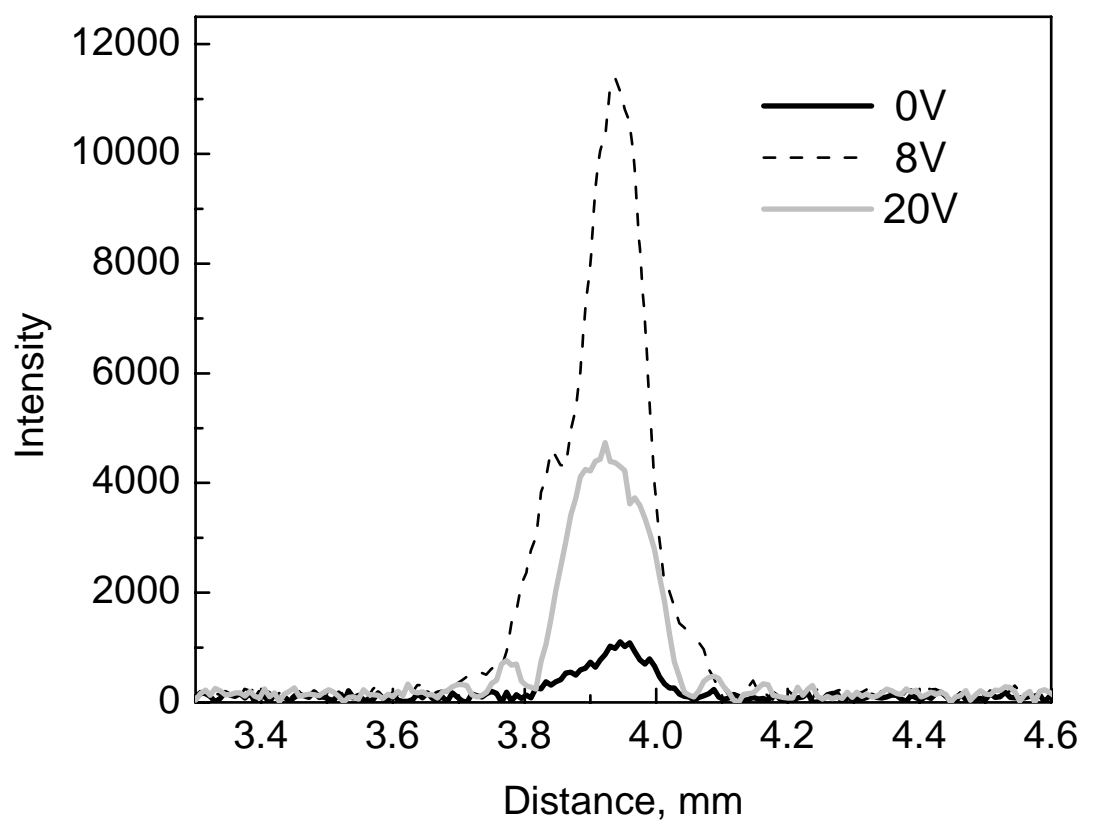

Figure 3.16: Beam intensity profiles at (a) $V=0$, (b) $8 \mathrm{~V}_{\mathrm{rms}}$, and (c) $20 \mathrm{~V}_{\mathrm{rms}}$. The diameter of the Fresnel zone plate is $1 \mathrm{~cm}$ and the focal length is $50 \mathrm{~cm}$.

Due to the polymer network effect, ${ }^{63}$ the PNLC Fresnel lens has fast response time. The rise and decay time were measured between 0 and $8 \mathrm{~V}_{\mathrm{rms}}$ and results are 4.4 and $9.1 \mathrm{~ms}$, respectively, for the 5- $\mu \mathrm{m}$ PNLC (5 wt \% monomer) Fresnel lens. Increasing monomer concentration would lead to a stronger anchoring force between the polymer network and LC interfaces. As a result, the response time can be even faster. The tradeoff is that the required operating voltage increases proportionally.

Figure 3.17 shows the measured diffraction efficiency as a function the applied voltage. The maximum diffraction efficiency is approximately $23 \%$. This value is close to the diffraction efficiency of $25.6 \%$ for the photomask alone. The slightly lower 
diffraction efficiency might be due to the defects of the polymer network at the zone edges, which results in molecular disorder. A small light scattering was observed at the zone edges.

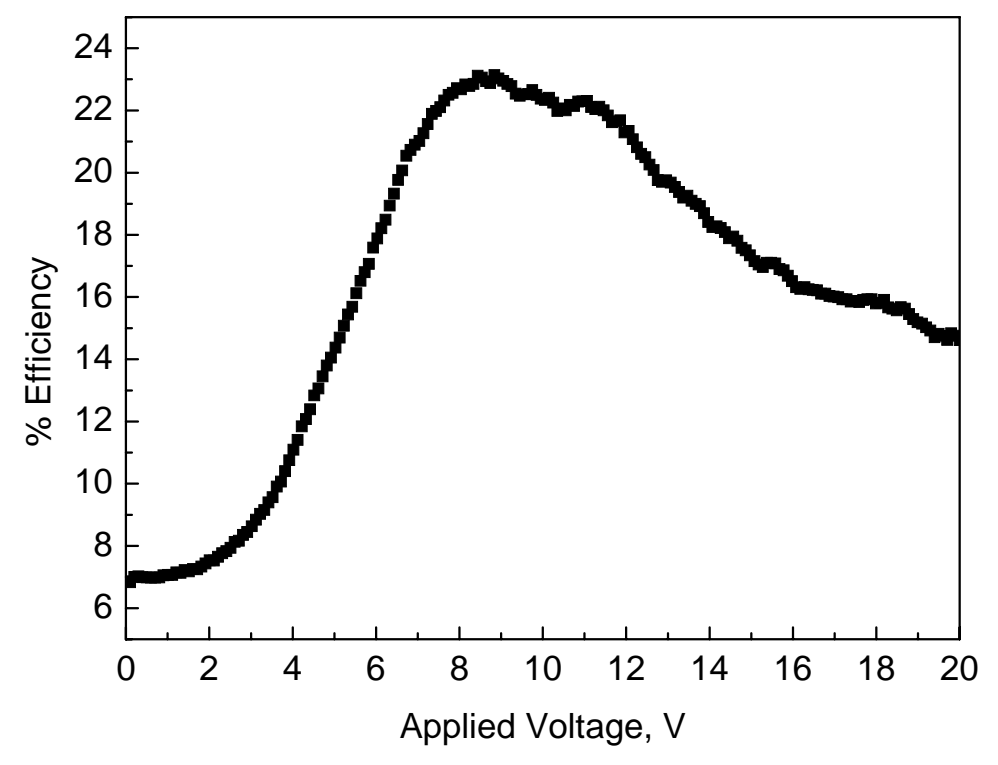

Figure 3.17: The voltage-dependent diffraction efficiency of the PNLC Fresnel lens. LC: MLC-6252, cell gap $d=5 \mu \mathrm{m}$, and monomer concentration $\mathrm{c}=5 \%$.

\subsubsection{Conclusion}

A switchable Fresnel zone plate lens is demonstrated using a polymer network liquid crystal. The fabrication process is relatively simple. A slight diffraction effect is observed at voltage-off state due to the inhomogeneous polymer network concentration. The diffraction effect of the PNLC Fresnel lens can be controlled by a homogeneous 
electric field. A continuously change in diffraction efficiency is obtained. The maximum diffraction efficiency of the PNLC Fresnel lens is measured to be $23 \%$. The required operating voltage is below $10 \mathrm{~V}_{\text {rms. }}$. Due to the strong anchoring force of polymer network, the response time is fast around $5 \mathrm{~ms}$. A good focusing effect is obtained for the -1 order (primary focal length). The imaging property is investigated. A relatively good image is obtained. Such a device works well for a linearly polarized light.

\subsection{Fresnel Lens Using Phase-Separated Composite Film (PSCOF)}

\subsubsection{Experimental Methods}

This phase-separated composite film (PSCOF) method can be used to prepare multilayer structures. It yields adjacent uniform layers of the LC and polymer. The configuration of the optic axis in the LC layer can be controlled with an alignment layer on the substrate closest to the LC layer. The operation of a PSCOF device relies on changes in its birefringence in response to an applied electric field, as in conventional displays. This technique keeps the systems with operating voltage low and free from light scattering.

Figure 3.18 shows the schematic drawing of the fabrication method. The same photomask with Fresnel zone pattern for fabricating nanoscale PDLC and PNLC Fresnel lens is used here. The innermost zone has radius $r_{1}=0.5 \mathrm{~mm}$ and the $n$th zone has radius $r_{n}$ which satisfies $r_{n}^{2}=n r_{1}^{2} ; n$ is the zone number. Our zone plate consists of 80 concentric rings within $1 \mathrm{~cm}$ diameter. 
To fabricate PSCOF zone plate, we mixed 70\% UV curable prepolymer NOA 65 (Norland) with $30 \%$ nematic LC E48 (Merck, $\Delta n=0.231$ ). The E48/NOA65 mixture was injected to a homogeneous cell comprising of ITO (indium-tin-oxide)-coated glass substrates. The inner surfaces of the ITO-glass substrates were coated with thin polyimide layers and rubbed in anti-parallel directions. The pretilt angle is $\sim 3^{\circ}$ and cell gap is $\mathrm{d} \sim 16 \mu \mathrm{m}$. During exposure, the cell was in proximity contact with the photomask. Phase separation was carried out at a temperature of around $100{ }^{\circ} \mathrm{C}$. The UV light with intensity $\mathrm{I} \sim 20 \mathrm{~mW} / \mathrm{cm}^{2}$. was used to cure the cell from the photomask side for 15 minutes.

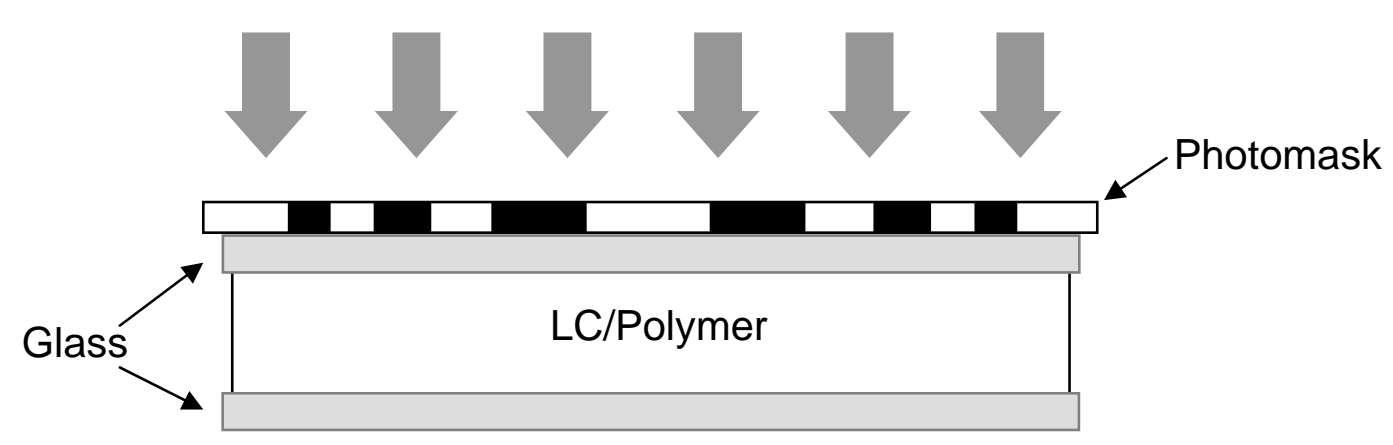

Figure 3.18: Schematic representation of the fabrication process of the PSCOF Fresnel lens.

During UV (Loctite model 98016) exposure, the photomask was in proximity contact with the LC cell. As shown in Figure 3.18, the odd zones are transparent and even zones are opaque. Thus, polymerization process would first take place in the odd zones resulting in a surface-relief structure corresponding to the photomask pattern. Although 
even zones are opaque, polymerizations could still proceed to these regions. The even zones have a lower polymer concentration so that the polymer layer in the even zones has thinner thickness. Their threshold voltage is lower. A PSCOF replicating zone plate configuration is thus formed.

\subsubsection{Operation Principle}

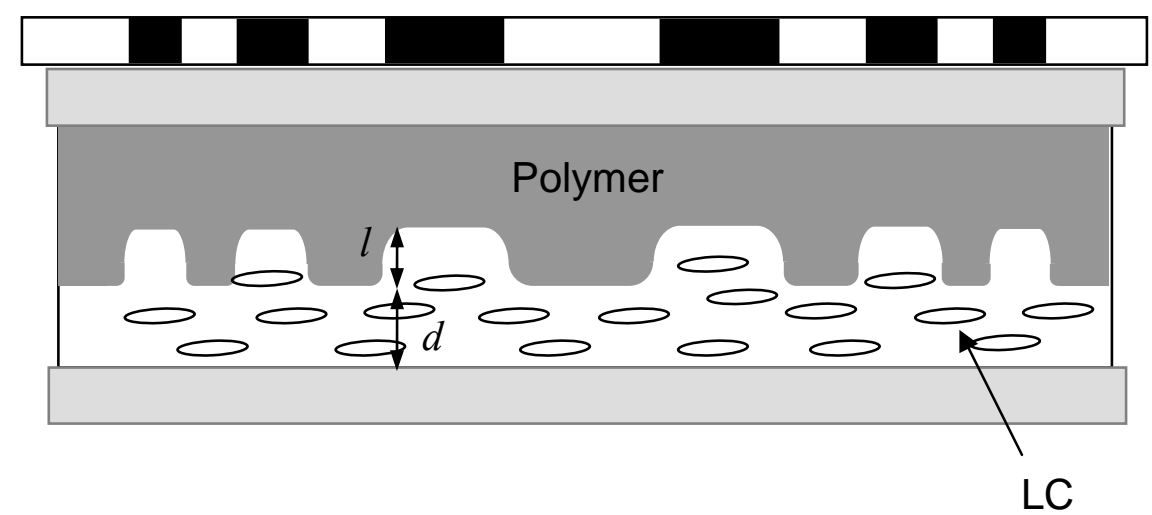

Figure 3.19: Cross-sectional model of the PSCOF Fresnel lens.

Unlike the polymer network liquid crystal and PDLC devices, the prepared PSCOF Fresnel lens sample is highly transparent in the voltage-on and -off state. Figure 3.19 shows the schematic diagram of PSCOF Fresnel zone plate structure. After polymerization process, the LC layer is formed and completely separated from the polymer layer. The cured polymer layer adheres to the glass substrate of the UV light incident side. Since the polymerization starts from the UV irradiation area, the transparent zones of the photomask may have a thicker polymer layer. The thicker 
dielectric polymer layer reduces the voltage drop across the LC layer. Therefore, the odd zones with a thicker polymer layer would require a higher voltage to reorient LC molecules. In the low voltage regime, the LC molecules in the even zones are reoriented first while the molecules in the odd zones remain in the original alignment owing to their higher threshold voltage. A binary-phase Fresnel lens is thus formed.

The LC molecules are homogeneously aligned although there is only one side of the substrates has the buffed polyimide layer. Figure 3.20 shows a micrograph of the PSCOF Fresnel lens sample. The cell is observed under crossed polarizers, where the rubbing direction of the liquid crystal cell is parallel to the polarization direction of the polarizer. Some light leakage is observed between two neighboring zones, because the polymer layer is not sharp enough between two zones. It is difficult for the LC molecules to follow the alignment direction of the polyimide layer. The rest of the observed region is dark. It indicates that a relatively good homogenous alignment has been obtained along the rubbing direction of the substrate.

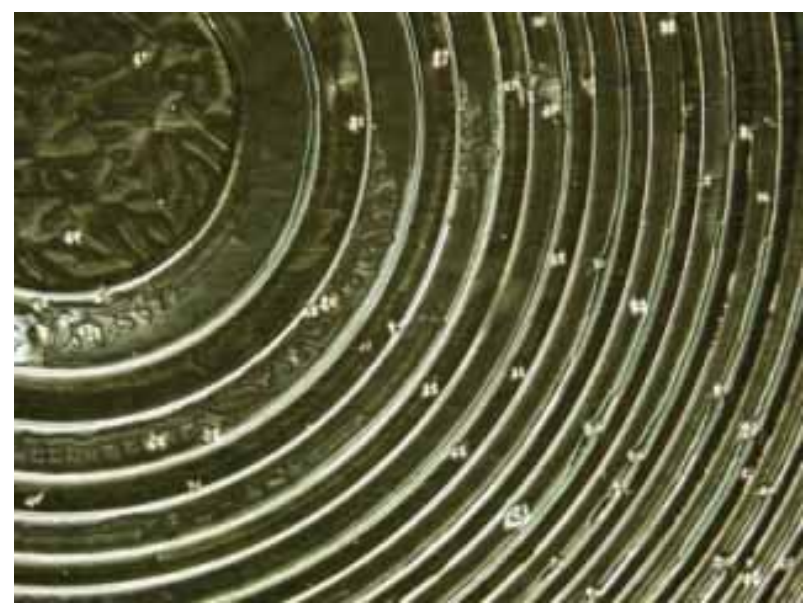

Figure 3.20: Microscope images of the PSCOF Fresnel lens cell at $V=0$. The sample is sandwiched between two crossed polarizers. 
If the polarization of the incident light is parallel to the rubbing direction of the cell, the phase difference between the neighboring zones is

$$
\Delta \delta=\frac{2 \pi}{\lambda}\left[n_{\text {eff }, \text { even }}(l+d)-\left(n_{\text {eff.odd }} \cdot d+n_{p} \cdot l\right)\right]
$$

where $\Delta \delta$ is the phase difference of the effective refractive indices in the adjacent zones, $d$ and $l$ is indicated in Fig. 3.19. After polymerization, the thickness of polymer layer is different spatially due to the different UV intensity in even and odd zones. The thickness difference of polymer layer between odd and even zones is $l$. $d$ is the thickness of liquid crystal layer in odd zones. $n_{\text {eff,even }}$ and $n_{\text {eff, odd }}$ is the extraordinary refractive index of liquid crystal in even and odd zones, respectively. $n_{p}$ is the refractive index of polymer. $\lambda$ is the wavelength of the incident beam. The phase retardation is different between even and odd zones at voltage-off state, since the light propagates through different thickness of polymer and liquid crystal layer. When an electric field is introduced, the liquid crystal molecules will be reoriented. The effective extraordinary index will be changed as well. Therefore, the induced phase shift is electrically tunable. The phase change corresponds to color change when observed from an optical microscope through crossed polarizers. Under this visual inspection, the rubbing direction of the $\mathrm{LC}$ cell was oriented at $45^{\circ}$ with respect to the optical axis of the linear polarizer. The optical micrographs of the PSCOF Fresnel lens were taken at different voltages. Results are shown in Fig. 3.21. 


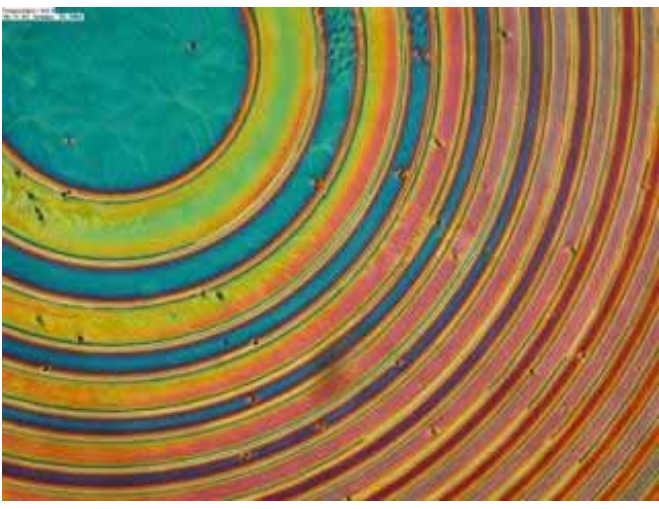

(a)

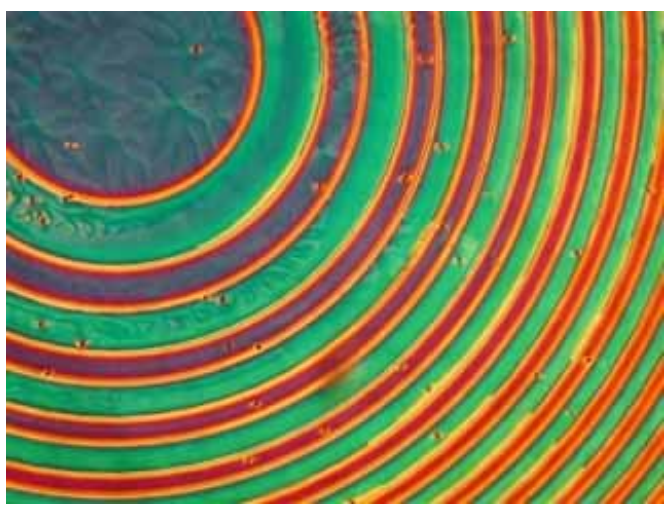

(c)

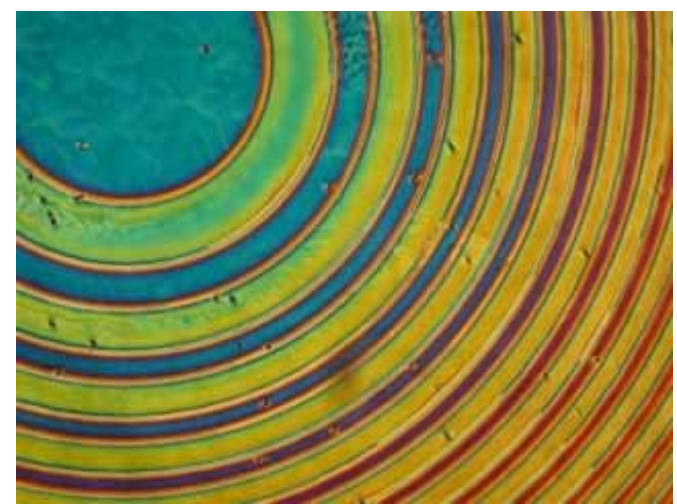

(b)

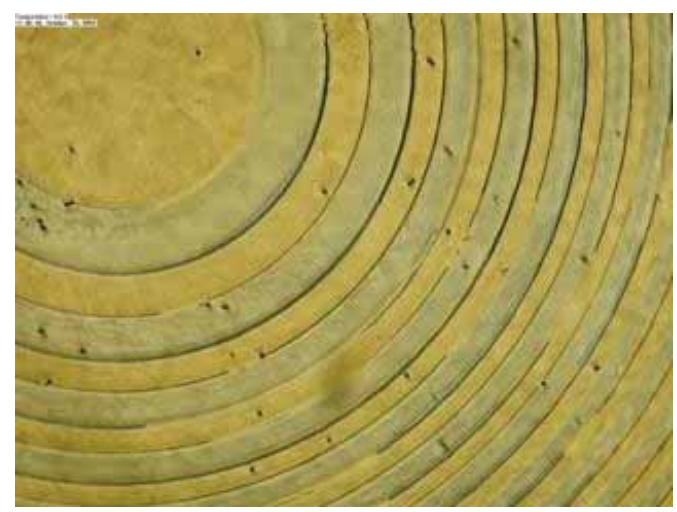

(d)

Figure 3.21: Microscope images of the PSCOF Fresnel lens at (a) $V=0$, (b) $4 \mathrm{~V}_{\mathrm{rms}}$, (c) 6 $\mathrm{V}_{\mathrm{rms}}$, and (d) $16 \mathrm{~V}_{\mathrm{rms}}$. The LC cell is sandwiched between two crossed polarizers. Cell gap $d=16 \mu \mathrm{m}$.

Figures 3.21(a-d) show a portion of the LC zone plate at $V=0,4,6$ and $16 \mathrm{~V}_{\mathrm{rms}}$, respectively. At $V=0$, the sample presents the Fresnel zone structure, because the odd and even zones exhibit different phases. The color shifts of each odd or even zone are due to the non-uniformity of the cell gap. As the voltage exceeds a threshold $\left(\sim 2.3 \mathrm{~V}_{\mathrm{rms}}\right)$, the LC molecules in even zones start to be reoriented first, as shown in Figures 3.21(b-d). The color change of the odd and even zones results from the refractive index change. In 
the higher voltage regime $\left(\sim 16 \mathrm{~V}_{\mathrm{rms}}\right)$, the bulk LC directors are orientated nearly perpendicular to the substrates, thus the zone structure is gradually erased.

A drawback of the PSCOF Fresnel lens is that it is polarization sensitive. For the light polarization perpendicular to the rubbing direction, it encounters the ordinary refractive index $n_{o}$ of the liquid crystal layer. To overcome the polarization dependence, stacking two orthogonal homogeneous PSCOF zone plates could be considered.

\subsubsection{Experimental Results and Discussion}

The primary focal length $f_{11}$ is related to the innermost radius $r_{1}$ as $f_{1}=r_{1}^{2} / \lambda$ for a binary-phase Fresnel lens. The primary focal length of our LC Fresnel lens was estimated to be $50 \mathrm{~cm}$ for the He-Ne laser employed $(\lambda=632.8 \mathrm{~nm})$. Fresnel zone lens normally has multiple foci due to the higher-order Fourier components. The foci are at $f, f / 3, f / 5, \ldots$ Most of the incident light is diffracted into the primary focus. The diffraction efficiency $\left(\eta_{\mathrm{n}}\right)$ of these foci is $\eta_{\mathrm{n}}=\operatorname{sinc}^{2}(\mathrm{n} / 2), \mathrm{n}= \pm 1, \pm 3, \pm 5, \ldots$ The theoretical diffraction efficiency of the primary focus is $41 \%$.

To evaluate the image quality of the PSCOF Fresnel lens, a resolution chart was used. Figure 3.22 shows the image properties of our sample. A lens testing system OS100 from Thorlab was used. The testing system includes a white light source and a collimator. A resolution chart (OSR1) was placed in front of the collimator. Our PSCOF Fresnel lens was put after the collimator. The CCD was located at about $55 \mathrm{~cm}$ away from the sample. When the maximum efficiency is achieved at $5 \mathrm{~V}_{\text {rms }}$, a clear image is obtained. Our Fresnel lens has a very good image quality and has a relatively clear image to the smallest 
chart of group 5, which corresponds to 32 lines per mm. At the minimum efficiency $\sim 12$ $\mathrm{V}_{\text {rms, }}$, the image is washed out, as shown in the right photo of Fig. 3.22.

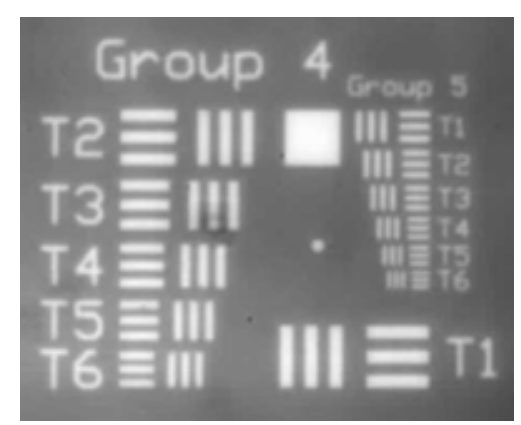

(a)

(b)

Figure 3.22: Image properties of the PSCOF Fresnel lens recorded by a CCD camera at (a) $5 \mathrm{~V}_{\text {rms }}$ and (b) $12 \mathrm{~V}_{\mathrm{rms}}$.

Figure 3.23 shows the measured diffraction efficiency as a function the applied voltage. When the applied voltage exceeds the threshold, the LC molecules state to be reoriented. The threshold voltage in the odd and even zones is different. Therefore, the LC tilt angles are different when a uniform electric field is applied. The phase difference between the odd and even zones changes while the LC tilt angle changes. The maximum diffraction efficiency is approximately $35 \%$ at $V=5 \mathrm{~V}_{\text {rms }}$. The minimum is obtained at $V$ $=12 \mathrm{~V}_{\mathrm{rms}}$. This value is close to the diffraction efficiency of $41 \%$ for the photomask alone. The slightly lower diffraction efficiency might be due to the defects of the polymer network at the zone edges, which results in molecular disorder. A small light scattering was observed at the zone edges. In the high voltage regime ( $\left.V>22 \mathrm{~V}_{\mathrm{rms}}\right)$, all the bulk LC molecules are rotated to be perpendicular to the substrate. Therefore, the diffraction efficiency is gradually decreased. 


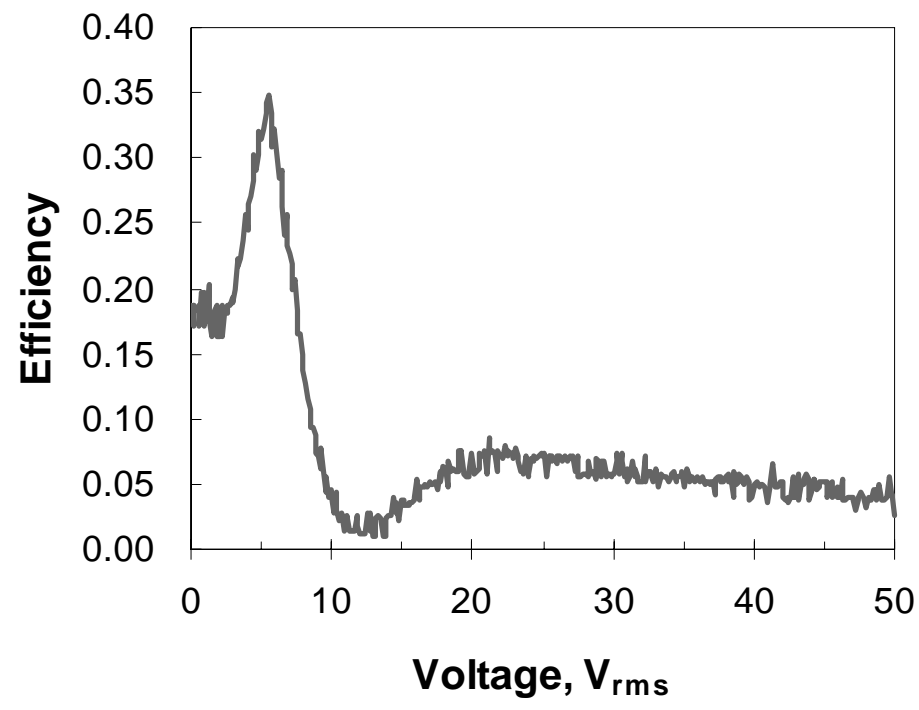

Figure 3.23: The voltage-dependent diffraction efficiency of the PSCOF Fresnel lens. The diameter of the Fresnel zone plate is $1 \mathrm{~cm}$ and the focal length is $50 \mathrm{~cm}$.

Figure 3.24 plots the measured 3-D laser intensity profiles of the outgoing beam using a digital CCD camera at different voltages. At $V=5 \mathrm{~V}_{\mathrm{rms}}$, the maximum diffraction efficiency occurs and the peak intensity is obtained. When the minimum efficiency is achieved, a slight focusing spot is observed at $V=12 \mathrm{~V}_{\text {rms. }}$. The rise and decay time of the PSCOF Fresnel lens were measured by switching the applied voltages between 5 and $12 \mathrm{~V}_{\mathrm{rms}}$ and results are 9.0 and $34.5 \mathrm{~ms}$, respectively. Increasing the monomer concentration or reducing the cell gap would lead to a thinner LC layer and, consequently, a faster response time. However, the respective tradeoff is the increased voltage and decreased phase change. 


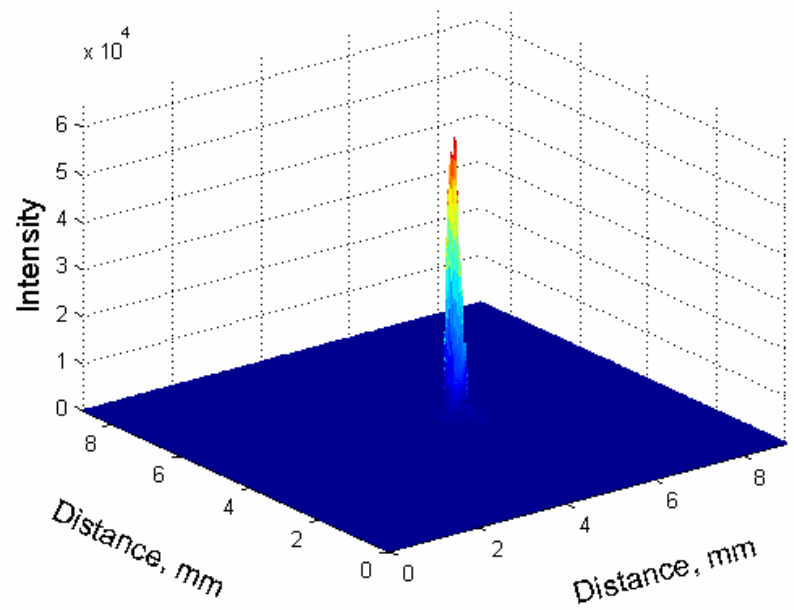

(a)

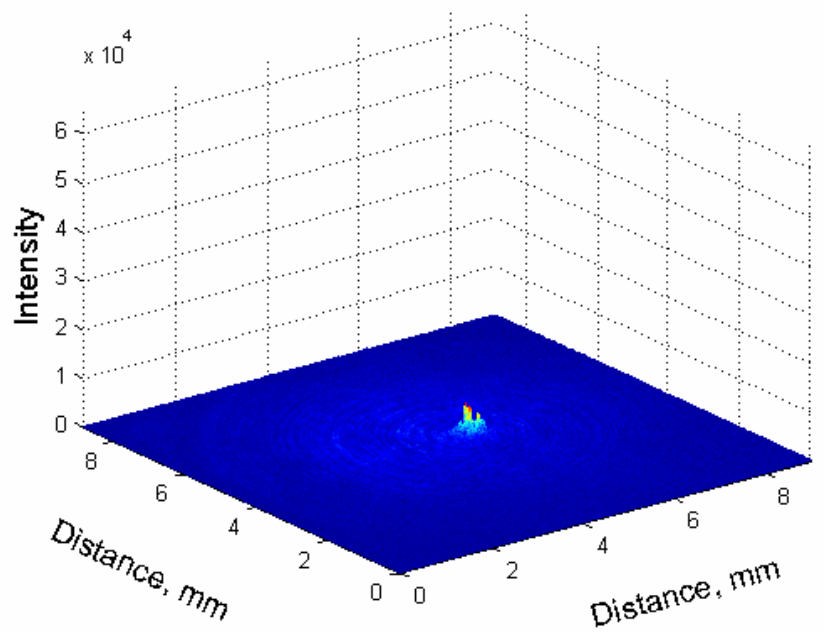

(b)

Figure 3.24: Beam intensity profile measured by the CCD camera at (a) $V=5 \mathrm{~V}_{\mathrm{rms}}$, and (b) $12 \mathrm{~V}_{\text {rms. }}$. The diameter of the Fresnel zone plate is $1 \mathrm{~cm}$ and the focal length is $50 \mathrm{~cm}$. 


\subsubsection{Conclusion}

A switchable Fresnel zone plate lens is demonstrated using a phase-separated composite film. The fabrication process is relatively simple. Since the LC and polymer are separated completely to become two layers. The sample has no light scattering and a relatively low operating voltage. This feature is different from other conventional LC/polymer composites. The diffraction properties of the PSCOF Fresnel lens were also investigated. The diffraction efficiency can be controlled continuously by a uniform electric field. A $35 \%$ diffraction efficiency was achieved at $V=5 \mathrm{~V}_{\mathrm{rms}}$ for a linear polarized light. The rise time and decay time were measured to be 9.04 and $34.5 \mathrm{~ms}$. 


\section{CHAPTER 4: TUNABLE-FOCUS FLAT LIQUID CRYSTAL SPHERICAL LENS}

\section{$\underline{4.1 \text { Introduction }}$}

Since liquid crystal (LC) was proposed for making a tunable lens, ${ }^{64}$ the LC based zoom lens has been studied extensively for machine vision, photonics, information

processing, and eye glasses. Various attempts such as surface relief profile, ${ }^{10,65,66}$ line or hole-patterned electrode, ${ }^{9,40,67,68}$. Fresnel zone type,${ }^{6,69}$ modal control, ${ }^{4}$ as well as polymer network LC technique ${ }^{7,70}$. have been demonstrated. Among these approaches, the lens with surface relief profile which combines a passive solid-state lens and a LC modulator possesses several merits, e.g., simple fabrication, single electrode, and easy to realize a spherical phase profile within the LC layer. However, the LC lens with curved inner surfaces would scatter light due to the poor molecular alignment. ${ }^{10}$. Moreover, its focus tunable range is rather limited. ${ }^{66}$

In this work, we demonstrated a flat LC lens exhibiting a wide-range tunable focal length. Unlike the surface relief LC lens, the present lens has planar substrates and a uniform LC layer. One of the flat substrates has an imbedded spherical electrode and the other has a planar electrode. The electric field from the spherical and planar electrodes induces a centrosymmetric gradient refractive index distribution within the LC layer which, in turn, causes the focusing effect. The focal length is tunable by the applied voltage. Unlike the nonuniform LC layer approach, our lens exhibits a uniform optical 
response across the lens aperture due to the homogeneous cell gap. No light scattering or diffraction occurs due to the homogeneous LC alignment and continuous electrode. Both positive and negative lenses can be realized by simply reversing the shape of the spherical electrode.

\subsection{Experimental Methods}

Figure 4.1 illustrates the fabrication procedures for a positive LC lens. The concave surface of the bottom glass substrate is coated with a transparent indium-tinoxide (ITO) electrode, as shown in Figure 4.1(a). Next, the sag area could be matched by a convex glass lens with the same curvature or filled with a polymer having the same refractive index as the employed glass substrate to form a planar substrate, as shown in Figure 4.1(b). For the demonstration purpose, we filled the sag area with an UV curable prepolymer. To simplify the fabrication process, we used an empty LC cell to seal the prepolymer. The glass substrate which is in contact with the prepolymer has $d_{2}=0.55$ $\mathrm{mm}$, but no ITO electrode. When the prepolymer was cured by UV, the lens and the LC cell were attached together. The inner surfaces of the LC cell are coated with polyimide alignment layers and rubbed in anti-parallel direction. The pretilt angle is $\sim 3^{\circ}$. When a LC mixture is injected into the cell, homogeneous alignment is induced by the buffed polyimide layers, as shown in Figure 4.1(c).

Based on the above-mentioned procedures, we fabricated a positive lens LC cell. The concave glass lens with radius $R=-9.30 \mathrm{~mm}$, aperture $D=6 \mathrm{~mm}$, and sag $d_{1}=0.34$ mm was purchased from Edmund Industrial Optics (BK7 glass, $\left.n_{\mathrm{g}}=1.517\right)$. The refractive index of the filled polymer NOA65 $\left(n_{\mathrm{p}} \sim 1.524\right.$, Norland Optical Adhesive) 
would affect the initial focal length of the LC lens. If $n_{p} \approx n_{g}$, then the LC device would not focus light in the voltage-off state. If $n_{p}$ is much smaller than $n_{g}$, then the device would have an initial focus. A high birefringence LC mixture formulated in our labs, designated as UCF-2 $(\Delta n=0.4$ at $\lambda=633 \mathrm{~nm}),{ }^{71}$. was used to fill the $40-\mu \mathrm{m}$-cell gap.
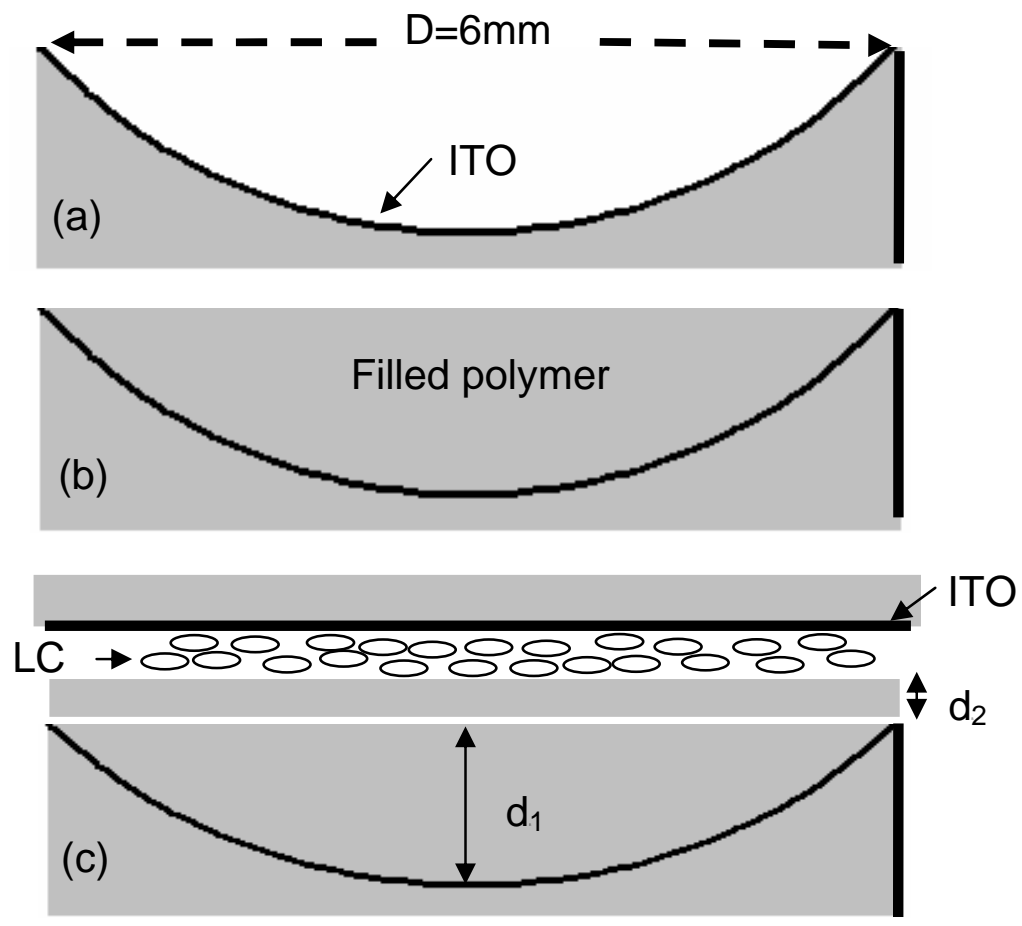

Figure 4.1: Procedures for fabricating the spherical LC lens: (a) deposit ITO on a concave glass lens, (b) fill the sag area with polymer, and (c) assemble the LC lens cell with another flat glass substrate.

\section{$\underline{4.3 \text { Experimental Results and Discussions }}$}

To evaluate the optical properties of the spherical lens, we investigated the profile of the phase retardation by observing the interference fringes between the ordinary and extraordinary rays using a white light under crossed polarizers. The rubbing direction of 
the lens cell is oriented at $45^{\circ}$ with respect to the fast axis of the linear polarizer. Two images of the lens cell at $V=0$ and $V=25 \mathrm{~V}_{\text {rms }}$ were taken using a digital camera, and results are shown in Figs. 4.2(a) and 4.2(b), respectively.

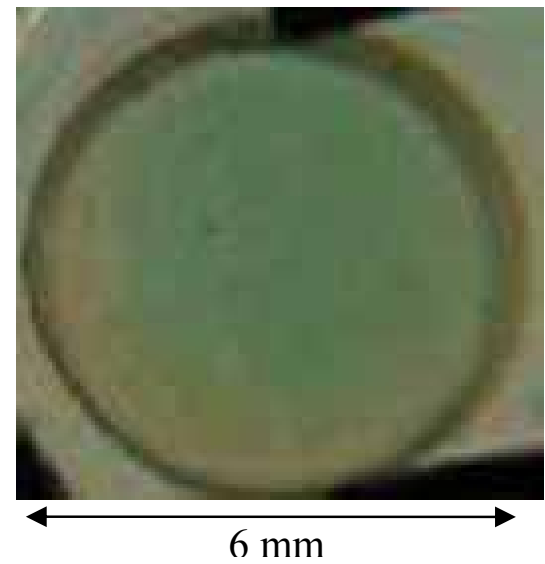

(a)

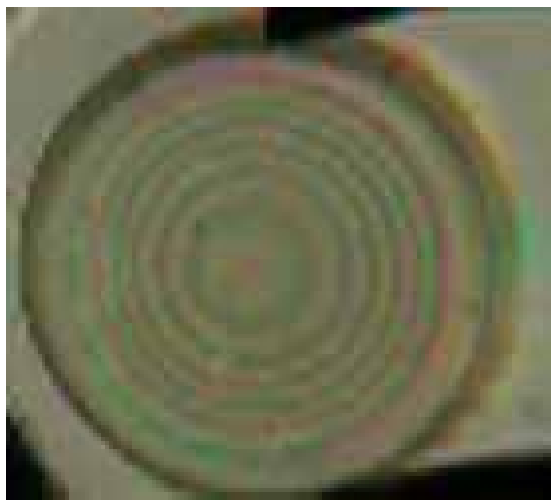

(b)

Figure 4.2: Interferograms of the circular LC lens at two different operating voltages: (a) $V=0$, (b) $V=25 \mathrm{~V}_{\mathrm{rms}}$. The lens aperture $D=6 \mathrm{~mm}$. The polarizers are crossed. The rubbing direction of the cell is oriented at $45^{\circ}$ with respect to the fast axis of the linear polarizer. LC used is UCF-2 and cell gap $d=40 \mu \mathrm{m}$.

At $V=0$, the sample is optically homogeneous and no interference rings are observed, as shown in Figure 4.2(a). This means that the gradient refractive index distribution does not occur in the LC layer. When a voltage is applied to the lens cell, concentric circular rings appear. Figure 4.2(b) shows the image taken at $V=25 \mathrm{~V}_{\mathrm{rms}}$. As the applied voltage is increased, the circular rings start to occur from the borders and then gradually expand to the center. This implies that a gradient refractive index is induced in the LC layer and is tunable by the applied voltage. 

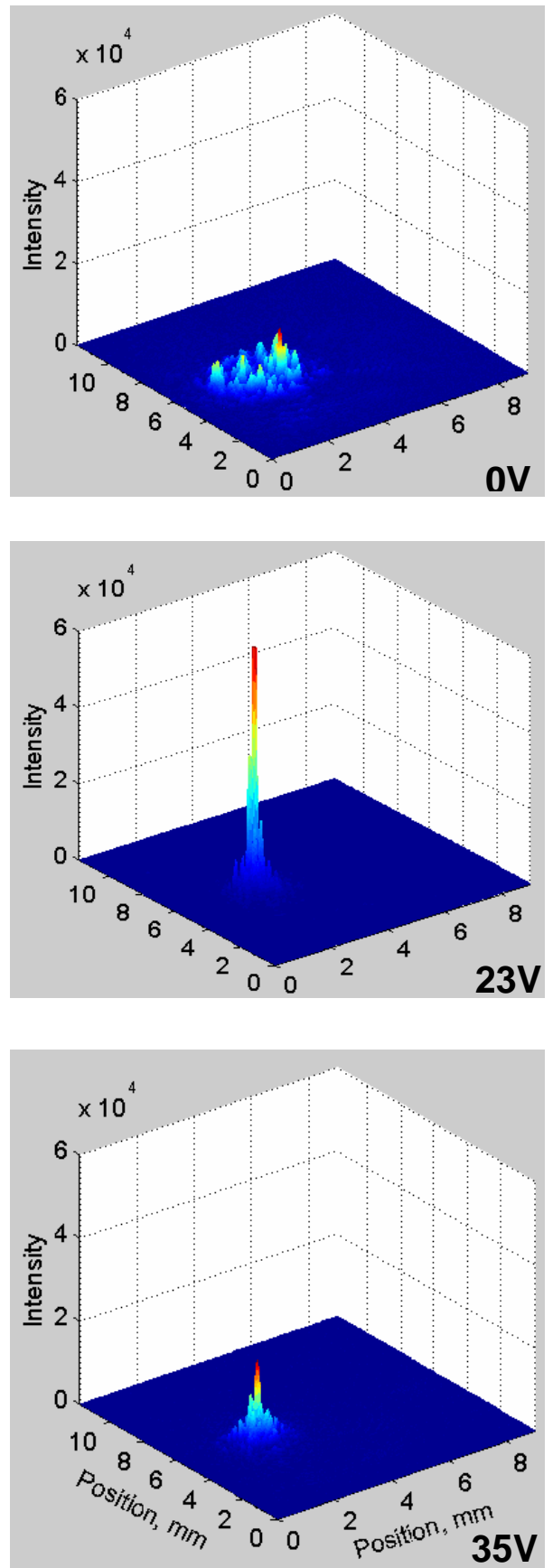

Figure 4.3: CCD images of the measured He-Ne laser beam intensity profile at $V=0,23$, and $35 \mathrm{~V}_{\mathrm{rms}}$, respectively. 
The movement direction of the rings towards the center indicates that the LC in the central area experiences a weaker electric field than that in the borders. This phenomenon basically correlates to the spherical electrode gap. At a given voltage, the electric field in the center of the LC lens is weaker than that in the edges. Further increasing the applied voltage, the numbers of the circular rings increase sharply and then decrease gradually in the high voltage regime where most of the LC directors are reoriented by the electric field.

To characterize the light focusing properties of the lens cell, we measured the 3-D profiles of the outgoing beams (He-Ne laser, $\lambda=633 \mathrm{~nm}$ ) using a CCD camera. The CCD camera was set at $\sim 80 \mathrm{~cm}$ behind the LC lens cell. The intensity profiles were measured at $V=0,23$ and $35 \mathrm{~V}_{\mathrm{rms}}$, and results are shown in Figure 4.3. At $V=0$, the observed He-Ne laser beam is not very uniform due to the Gaussian nature of the input laser beam. The peak intensity is $\sim 6 \times 10^{3}$ arbitrary units. As the voltage increases to 23 $\mathrm{V}_{\mathrm{rms}}$, the focusing effect manifests. The measured intensity at the CCD focal plane exceeds $6.5 \times 10^{4}$ arbitrary units. As the voltage is further increased, the peak intensity of the outgoing beam tends to decrease. At $V=35 \mathrm{~V}_{\text {rms }}$, the peak intensity drops to $1.7 \times 10^{4}$ arbitrary units. This is because the LC molecules in the bulk are reoriented by the electric field. The curvature of the refractive index profile is gradually flattened. As a result, the focal length of the lens increases and the measured light intensity at the CCD focal plane decreases. 


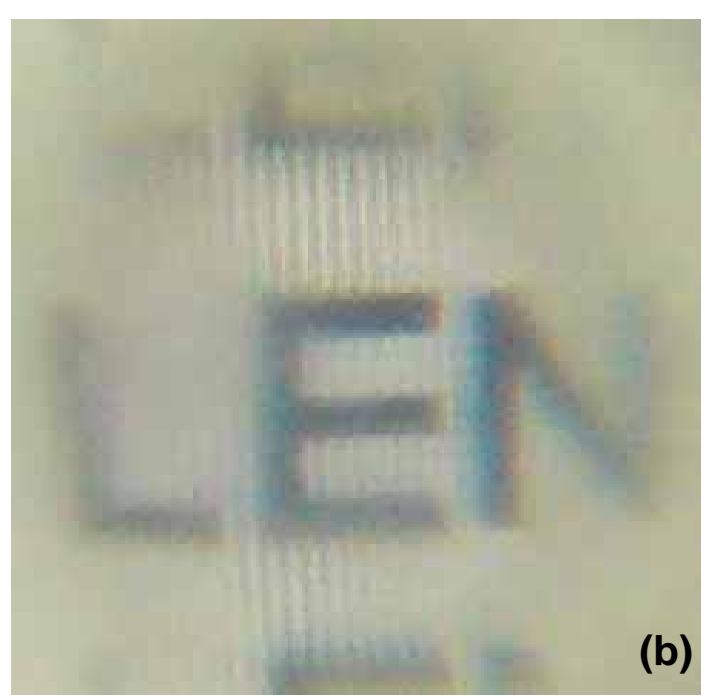

Figure 4.4: Imaging behavior of the LC lens at (a) $V=0$ and (b) $V=30 \mathrm{~V}_{\text {rms. }}$

To evaluate the image quality of the LC lens, we typed a few lines of "LENS" on a computer screen as an object and the lens was set at $\sim 15 \mathrm{~cm}$ in front of the computer screen. Since the LC lens works for a linearly polarized light, a sheet linear polarizer was placed between the sample and the screen. The rubbing direction of the LC cell was adjusted to be parallel to the polarizer's transmission axis. A digital camera was fixed 
right behind the sample. Two images were taken at $V=0$ and $V=30 \mathrm{~V}_{\text {rms }}$, as shown in Figures 4.4(a) and 4.4(b), respectively. At $V=0$, a clear image of the typed words is observed. As the applied voltage increases, the observed image is magnified and slightly blurred. The blurred image is mainly due to the defocusing effect. As the applied voltage exceeds $40 \mathrm{~V}_{\text {rms }}$, the image size is gradually reduced. The larger image means the focal length of the LC lens is longer than the distance between the sample and the object. The magnified image that we observed is actually a virtual image.

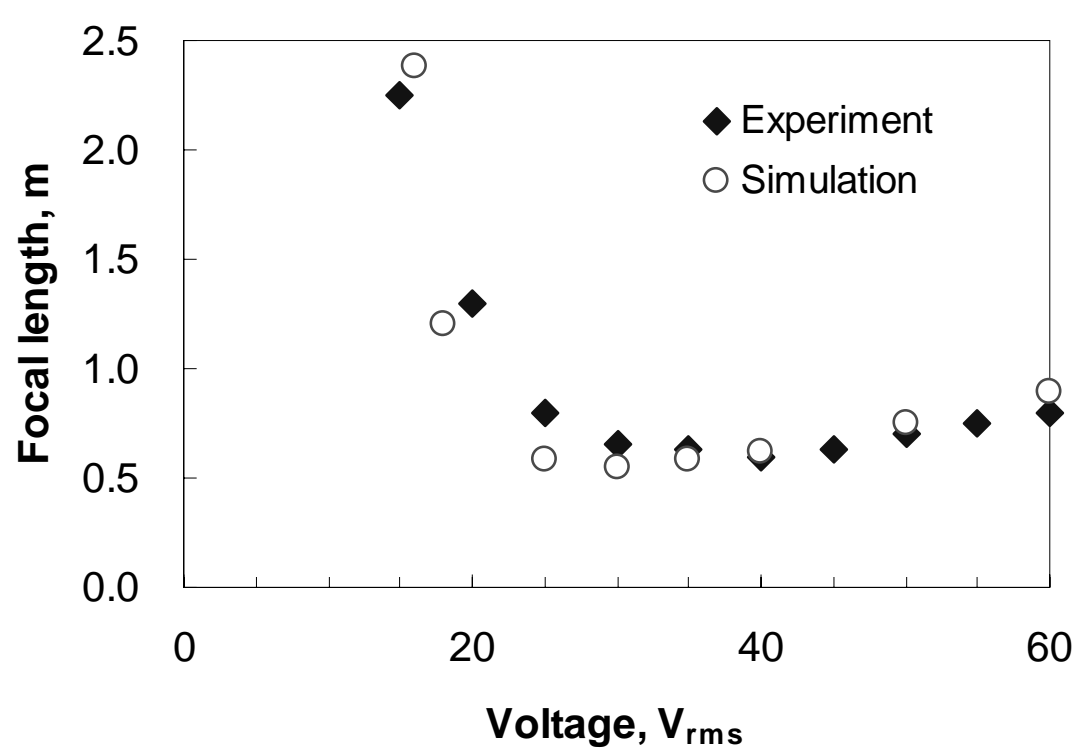

Figure 4.5: Voltage-dependent focal length of the LC lens. Lens aperture $D=6 \mathrm{~mm}, \mathrm{LC}$ : UCF-2, cell gap $d=40 \mu \mathrm{m}$ and $\lambda=633 \mathrm{~nm}$. Closed squares and open circles represent the experimental and simulation results, respectively.

The voltage-dependent focal length of the lens was investigated and results are plotted in Figure 4.5. At $V=0$, LC directors are aligned homogeneously due to the 
surface anchoring effect from the substrates. Thus, no focusing effect occurs or the focal point is at infinity. As the voltage increases, the focal length is reduced accordingly. At $V$ $\sim 40 \mathrm{~V}_{\mathrm{rm}}$, the focal length reaches a minimum $(f \sim 0.6 \mathrm{~m})$. Further increasing the voltage would cause the focal length to bounce back, but at a different rate.

\subsection{Numerical Calculation and Focal Length Prediction}

The focal length of an LC lens can be evaluated using the Fresnel's approximation:

$$
f=\frac{r^{2}}{2 \delta n d_{L C}},
$$

where $r=D / 2$ ( $D$ is the lens aperture), $d_{\mathrm{LC}}$ is the LC layer thickness, and $\delta n$ is the refractive index difference between the lens center and border. From Figure 4.1, $\delta n$ is determined by the electric field difference between the lens center and the border. When a voltage $V$ is applied to the lens cell, the electric field in the center $\left(E_{\text {center }}\right)$ and at the border $\left(E_{\text {border }}\right)$ is expressed as:

$$
\begin{gathered}
E_{\text {center }}=\frac{V / \varepsilon_{L C}}{\frac{d_{L C}}{\varepsilon_{L C}}+\frac{d_{2}}{\varepsilon_{2}}+\frac{d_{1}}{\varepsilon_{1}}}, \\
E_{\text {border }}=\frac{V / \varepsilon_{L C}}{\frac{d_{L C}}{\varepsilon_{L C}}+\frac{d_{2}}{\varepsilon_{2}}}
\end{gathered}
$$

where $\varepsilon_{\mathrm{LC}}, \varepsilon_{2}$, and $\varepsilon_{1}$. represent the dielectric constant of the LC, medium 2 and 1 , respectively. In an ideal case, we would like to eliminate the glass substrate which is closer to the spherical electrode, i.e., $d_{2} \sim 0$. In such a condition, the electric field shielding effect due to the glass substrate is reduced and the required operating voltage is 
lowered. From Eq. (4.1), the shortest focal length occurs when $\delta n=\Delta n$, i.e., the LC molecules in the border are completely aligned by the electric field while those in the center are not yet reoriented owing to the weaker electric field.

To explain this focal length transition phenomenon quantitatively, we calculate the voltage-induced LC director reorientation numerically. We start by calculating the voltage-dependent refractive index change for a symmetric, uniform liquid crystal layer and use that to predict the voltage-dependent focal length.

The LC directors are reoriented to be parallel to the electric field $(\boldsymbol{E})$ in order to minimize the free energy. The energy associated with the elastic forces can be described in terms of three elastic constants. The free energy of nematic molecules in the static electric field $\boldsymbol{E}$ is generally expressed by the Oseen-Frank elastic free energy density and electric free energy. ${ }^{14}$ The elastic free energy density of a nematic can be expressed as:

$$
\Delta F_{\text {elastic }}=\frac{1}{2} k_{11}(\nabla \cdot \mathbf{n})^{2}+\frac{1}{2} k_{22}(\mathbf{n} \cdot \nabla \times \mathbf{n})^{2}+\frac{1}{2} k_{33}(\mathbf{n} \times \nabla \times \mathbf{n})^{2},
$$

where $\boldsymbol{n}=\left(n_{x}, n_{y}, n_{z}\right)$ is the LC director vector, $k_{11}, k_{22}$ and $k_{33}$ are elastic constants associated with splay, twist and bend deformations. The electric free energy is related to dielectric constants of the LC and the applied electric field as

$$
\Delta F_{\text {electric }}=-\frac{1}{2}\left[\varepsilon_{\perp} \mathbf{E}^{2}+\Delta \varepsilon(\mathbf{n} \cdot \mathbf{E})^{2}\right]
$$

where $\Delta \varepsilon$, the dielectric anisotropy, is the difference between the dielectric constant parallel to the director, $\varepsilon_{/ /}$, and the dielectric constant perpendicular to the director, $\varepsilon_{\perp}$, i.e. $\Delta \varepsilon=\varepsilon_{/ /}-\varepsilon_{\perp}$ 
In our case, the electric field is uniform and only in the $z$ direction, i.e. $E_{x}=E_{y}=0$. Therefore, $\theta=\theta(z)$ and $\phi=\phi(z)$ are only dependent on $z$ and independent in $x$ and $y$ direction.

$$
\frac{\partial n_{l}}{\partial x}=\frac{\partial n_{l}}{\partial y}=0, l=x, y, z
$$

The total free energy density is reduced to

$$
\begin{aligned}
\Delta F_{\text {total }} & =\frac{1}{2}\left(k_{11} \cos ^{2} \theta+\frac{1}{2} k_{33} \sin ^{2} \theta\right)\left(\frac{d \theta}{d z}\right)^{2} \\
& +\frac{1}{2}\left(k_{22} \cos ^{2} \theta+k_{33} \sin ^{2} \theta\right) \cdot \cos ^{2} \theta\left(\frac{d \phi}{d z}\right)^{2} \\
& -\frac{1}{2} \frac{D_{z}^{2}}{\varepsilon_{0}\left(\varepsilon_{\perp}+\Delta \varepsilon \sin ^{2} \theta\right)},
\end{aligned}
$$

where $D_{z}=\varepsilon_{0}\left(\varepsilon_{\perp}+\Delta \varepsilon \sin ^{2} \theta\right) E_{z}$ is the displacement current. For a homogeneous LC cell, $\phi=90^{\circ}$, the total free energy density becomes

$$
\begin{aligned}
\Delta F_{\text {total }} & =\frac{1}{2}\left(k_{11} \cos ^{2} \theta+\frac{1}{2} k_{33} \sin ^{2} \theta\right)\left(\frac{d \theta}{d z}\right)^{2} \\
& -\frac{1}{2} \frac{D_{z}^{2}}{\varepsilon_{0}\left(\varepsilon_{\perp}+\Delta \varepsilon \sin ^{2} \theta\right)} .
\end{aligned}
$$

The total Gibbs free energy of the system is expressed as:

$$
\begin{gathered}
G=\int_{0}^{d} \Delta F_{\text {total }} d z=\frac{1}{2} \int_{0}^{d} F\left(z, \theta, \frac{d \theta}{d z}\right) d z \\
F\left(z, \theta, \frac{d \theta}{d z}\right)=\left(k_{11} \cos ^{2} \theta+k_{33} \sin ^{2} \theta\right)\left(\frac{d \theta}{d z}\right)^{2}+\frac{D_{z}^{2}}{\varepsilon_{0}\left(\varepsilon_{\perp}+\Delta \varepsilon \sin ^{2} \theta\right)} .
\end{gathered}
$$


The director's distribution can be obtained by minimizing the Gibbs free energy per unit area of the system by using the Euler-Lagrange equation to find the minima of Eq. (4.9):

$$
\frac{\partial F}{\partial \theta}-\frac{d}{d z}\left[\frac{\partial F}{\partial\left(\frac{d \theta}{d z}\right)}\right]=0 .
$$

The LC director reorientation is calculated by a standard one dimensional finite element method. $^{72,73}$. The director distribution, $\theta(z)$, as a function of normalized cell thickness (z/d) is shown in Figure 4.6. ${ }^{74}$

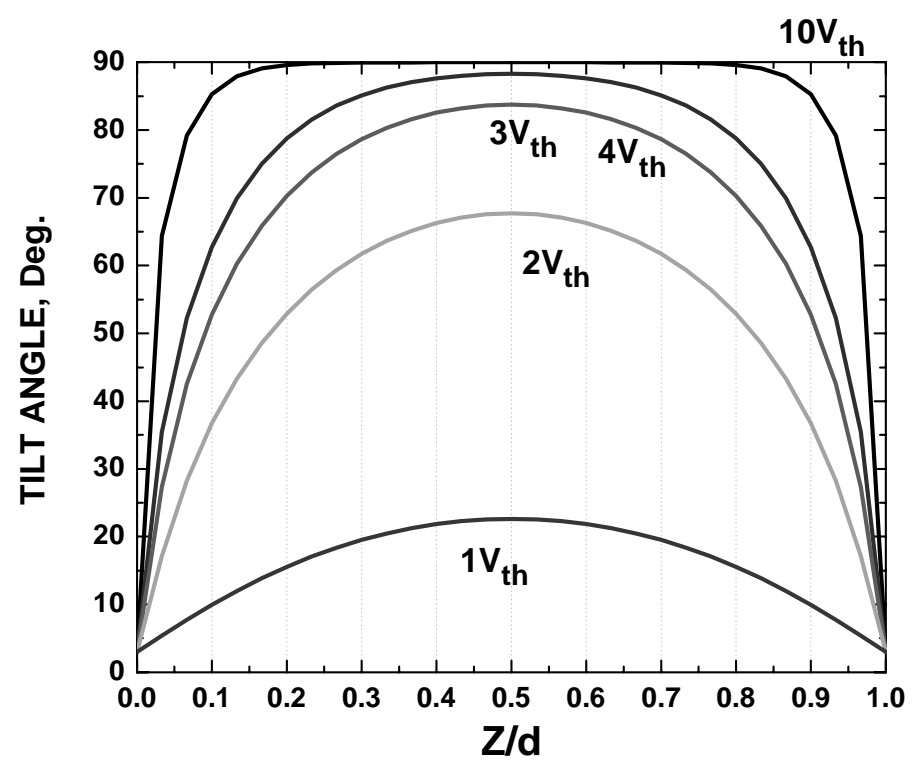

Figure 4.6: The LC directors distribution of a homogeneous cell at various normalized voltage $\left(V / V_{t h}\right)$. 
The tilt angle profile $\theta(z)$ can be used to calculate the average refractive index, $\tilde{n}$, of the liquid crystal at different applied voltages. For a given tilt angle the refractive index is given by the index ellipsoid equation ${ }^{75}$.

$$
n(z)=\frac{n_{e} n_{o}}{\sqrt{n_{o}^{2} \cos ^{2} \theta(z)+n_{e}{ }^{2} \sin ^{2} \theta(z)}}=\frac{n_{e} n_{o}}{\sqrt{n_{o}^{2}+\left(n_{e}{ }^{2}-n_{o}^{2}\right) \sin ^{2} \theta(z)}} .
$$

The tilt angle profile $\theta(z)$ is used in Eq. (4.12) to calculate the optical path, $\widetilde{n} d$, when light traverses through the cell. The average refractive index is expressed as

$$
\widetilde{n}=\int_{0}^{d} n(z) d z
$$

where $d$ is the cell gap.

Table 4.1 Physical parameters of UCF-2 for simulation

\begin{tabular}{|c|c|}
\hline UCF-2 parameters & \\
\hline Effective splay elastic constants, $k_{11 .}$ & $20.3 \mathrm{pN}$ \\
Effective bend elastic constants, $k_{33}$. & $33.8 \mathrm{pN}$ \\
Parallel dielectric constant, $\varepsilon_{/ /}$ & 14.9 \\
Perpendicular dielectric constant, $\varepsilon_{\perp}$ & 3.3 \\
Birefringence, $\Delta n$ & 0.4 \\
\hline
\end{tabular}


Using the equations and parameters, as shown in Table 4.1, of our homemade high birefringence liquid crystal (with pretilt angle, $\psi_{o}=2^{\circ}$ ), the voltage-dependent focal length of the lens was investigated and compared with the simulation results, as shown in Figure 4.5. Filled squares and open circles represent the experimental and simulation results, respectively, at $\lambda=633 \mathrm{~nm}$. The agreement is very good.

In comparison with other tunable lens technologies, the major advantages of our lens are in simple fabrication process, uniform LC cell gap, plano-substrate surface, and simple electrodes. In addition, the lens has a very wide range of tunable focal length without light diffraction and scattering. In theory, the light throughput can reach $100 \%$ for a linearly polarized laser beam. The response time of the lens depends on the LC cell gap. In our experiment, we used a high $\Delta n$ LC mixture and $40 \mu \mathrm{m}$ cell gap. The switching speed is $\sim 1 \mathrm{~s}$ at room temperature.

\section{$\underline{4.4 \text { Conclusion }}$}

A tunable-focus spherical lens using two flat substrates and inhomogeneous electric field over a homogeneous liquid crystal (LC) layer is demonstrated. The top flat substrate has an imbedded spherical indium-tin-oxide (ITO) electrode and the bottom has a planar ITO electrode on its inner surface. The inhomogeneous electric field generates a centrosymmetric gradient refractive index profile within the LC layer which

causes the focusing behavior. The focal length of the LC lens can be tuned continuously from infinity to $0.6 \mathrm{~m}$ by the applied voltage.

In conclusion, we have demonstrated an electrically tunable-focus LC lens using the combination of spherical and planar electrodes. Without voltage, the focal length of 
the LC lens is at infinity due to the flat substrates and homogeneous LC alignment. As the applied voltage increases, the focal length is gradually drawn near from infinity to $\sim 0.6 \mathrm{~m}$. By reversing the shape of the spherical electrode, a negative lens or lens arrays can be easily fabricated. 


\section{CHAPTER 5: LIQUID CRYSTAL MICROLENS ARRAYS WITH SWITCHABLE POSITIVE AND NEGATIVE FOCAL LENGTHS}

\section{$\underline{5.1 \text { Introduction }}$}

Liquid crystal (LC) has been demonstrated for making tunable-focus lenses and microlens arrays. Microlens array have been attractive and investigated for various applications such as optical interconnections, optical fiber switches, shutters of optical super-resolution devices, light deflection devices and imaging processing. ${ }^{42,76-80}$. In 3-D display system based on integral photography (IP), the microlens array with dynamically variable image planes has been proposed to enhance the resolution of a real and virtual image and increase the perception of depth of image. ${ }^{81}$. In addition, it will be very helpful in displaying 3-D animated images. This technique requires a focal length to be variable. In conventional devices, this is commonly done by moving the lens array along the optical axis. This kind of system leads to a bulky device and requires extra mechanical elements for movement. The variable-focus lenses can be obtained by changing the shape of the lens or creating a gradient refractive index profile in the materials with uniform thickness. For LC microlens, a spatial distribution of refractive index can be induced and be varied continuously by applied voltage instead of mechanical movement. It would lead to a compact system and much faster response time. To fabricate LC lenses, several approaches such as surface relief profile, ${ }^{10,82,83}$. line or hole-patterned electrode, ${ }^{9,40,67,68}$. special shape electrode, ${ }^{66,84}$ modal control, ${ }^{4}$. and polymer network $\mathrm{LC}^{7,85}$. have been 
developed. The focal length of the LC lens depends on the lens radius, LC layer thickness, and its gradient refractive index. To obtain a short focal length, a relatively thick LC layer $(\sim 200-300 \mu \mathrm{m})$ is required. As a result, the response time is sluggish. A microlens made of polymer network can improve the response time, however, the polymer network multi-domain will cause light scattering in the voltage-on state. Another important issue of these LC microlenses is the formation of disclination lines due to the uneven substrate and hole-patterned electrodes. These disclination lines degrade the lens focusing properties and should be eliminated.

In this work, we demonstrate a LC microlens array whose focal length can be switched from positive to negative or vise versa by the applied voltage. The LC lens with spherical electrode ${ }^{66}$ can have a large aperture, however, its tunable focus range is rather limited and it can only function as a converging lens. In our lens array, we flatten the top spherical-electrode- imbedded substrate with a polymer layer while the bottom one has a planar electrode. A homogeneous aligned LC layer is sandwiched between the flattened top and bottom substrates. The top convex substrate and LC layer work together like a zoom lens. By tuning the refractive index profile of the LC layer, the focal length of the microlens array can be switched from positive to negative or vise versa. Unlike the surface relief LC lens, our lens consists of planar substrates and a uniform LC layer so that the disclination lines are completely eliminated.

To obtain fast response time, we employed a dual-frequency liquid crystal (DFLC) mixture developed in our labs. DFLC exhibits a crossover frequency $\left(f_{c}\right)$ where dielectric anisotropy $\Delta \varepsilon=0$. Below $f_{c}, \Delta \varepsilon$ is positive, while $\Delta \varepsilon$ changes sign when $f>f_{c}{ }^{86,87}$. During the turn-on and -off processes, ac voltage bursts with low and high 
frequencies are applied. As a result, fast rise and decay times are achieved. Using our high birefringence and low viscosity DFLC mixture, our microlens has $\sim 5 \mathrm{~ms}$ response time.

\subsection{Dual Frequency Effect}

The dual-frequency effect ${ }^{88}$. utilizes the fact that the dielectric anisotropy, $\Delta \varepsilon=\varepsilon_{/ /}-\varepsilon_{\perp}$, changes sign at high frequencies. In the low frequency regime, $\Delta \varepsilon>0$, the electric field-induced torque in the longitudinal (z-axis) direction is larger than that in the transversal direction so that the LC directors are deformed at $V>V_{t h}$. When the low frequency voltage is replaced by a high frequency voltage, the sign change of $\Delta \varepsilon$ results in a torque that is larger in the transversal (the initial alignment) direction. As a result, this high frequency burst acts to restore the LC directors. The time constant of this restoring force is greatly influenced by the dielectric anisotropy at high frequencies and by the applied high frequency voltage.

The dynamics of this field-induced director-axis orientation effect is described by the following equation for small deformation angle:

$$
k_{11} \frac{\partial^{2} \phi}{\partial z^{2}}+\left(\Delta \varepsilon_{l} E_{l}^{2}-\Delta \varepsilon_{h} E_{h}^{2}\right) \phi=\gamma_{1} \frac{\partial \phi}{\partial t},
$$

where $\Delta \varepsilon_{l, h}$ represents the dielectric anisotropy at low and high frequencies, respectively, and $E_{l, h}$ represents the corresponding electric field strength. In this equation, we have allowed the high frequency field to be present also during the on-stage, together with the low frequency field. The presence of the high frequency field modifies the response times and threshold voltage: ${ }^{89}$ 


$$
\begin{gathered}
\tau_{\text {rise }}=\frac{\gamma_{1}}{\Delta \varepsilon_{1} E_{1}{ }^{2}-\Delta \varepsilon_{h} E_{h}{ }^{2}-k_{11}\left(\frac{\pi}{d}\right)^{2}}, \\
\tau_{\text {decay }}=\frac{\gamma_{1}}{k_{11}\left(\frac{\pi}{d}\right)^{2}+\Delta \varepsilon_{h} E_{h}{ }^{2}}, \\
V_{t h}{ }^{2}=V_{t h}{ }^{2}(0)+\left(\frac{\Delta \varepsilon_{h}}{\Delta \varepsilon_{l}}\right) V_{h}{ }^{2},
\end{gathered}
$$

where $V_{t h}(0)=\pi \sqrt{k_{11} / \Delta \varepsilon_{l}}$ is the threshold voltage for the low frequency field alone. The new threshold increases proportionally to the ratio of $\Delta \varepsilon_{h} / \Delta \varepsilon_{l}$ and the high frequency voltage $\left(V_{\mathrm{h}}\right)$ applied to the cell. This is simply due to the fact that the low frequency field has to overcome the resistant torques from the elastic and high frequency field.

Eq. (5.3) shows that the decay time is governed by two factors: the intrinsic elastic torque (the first term in the denominator) and the high frequency field-induced torque (the second term). Due to the presence of the high frequency field, the decay time is improved by the factor

$$
\frac{\tau_{\text {decay }}\left(V_{h}\right)}{\tau_{\text {decay }}(0)}=\frac{1}{1+\left(\frac{V_{h}}{V_{h, t h}}\right)^{2}},
$$

where $V_{h, t h}=\pi \sqrt{k_{11} / \Delta \varepsilon_{h}}$. 


\subsection{Device Structure and Experimental Methods}

Figure 5.1 illustrates the structure of our DFLC microlens array. The convex surface of the top glass substrate was coated with a transparent indium-tin-oxide (ITO) electrode and then flattened by a polymer having a specific refractive index in order to obtain a desired focal length at the initial state. The polymer surface was rubbed in one direction with a cloth for aligning LC molecules. On the other hand, the bottom ITO glass substrate was coated with a thin polyimide alignment layer and rubbed in anti-parallel direction with respect to the top substrate. The two substrates are separated by $18 \mu \mathrm{m}$ Mylar spacers and hermetically sealed using ultraviolet-curable glue. The cell was filled with a DFLC mixture. Homogeneous alignment is induced by the buffed polyimide and polymer layers. The DFLC mixture we employed consists of some biphenyl esters and laterally difluorinated tolanes. Its physical properties are summarized as follows: crossover frequency $f_{c} \sim 5 \mathrm{kHz}$, birefringence $\Delta n \sim 0.285$ (at $\lambda=633 \mathrm{~nm}$ and $T=22{ }^{\circ} \mathrm{C}$ ), and dielectric anisotropy $\Delta \varepsilon=4.73$ at $f=1 \mathrm{kHz}$ and $\Delta \varepsilon=-3.93$ at $50 \mathrm{kHz}$.

As depicted in Figure 5.1, the top flattened substrate and LC layer work together as a zoom lens. The refractive index of the filled polymer NOA-76 $\left(n_{p} \sim 1.51, \varepsilon=3.33\right)$ plays an important role in affecting the initial focal length of the microlens. If the filled polymer has a similar refractive index to that of the top glass substrate (BK7, $\left.n_{g}=1.517\right)$, i.e. $n_{p} \approx n_{g}$, then the whole structure would have a uniform refractive index and not focus light in the voltage-off state. If we choose a polymer such that $n_{p} \neq n_{g}$, then the microlens would have an initial focus $f_{s}$ at $V=0$. In our design depicted in Figure 5.1, each single convex glass microlens has radius $R=450 \mu \mathrm{m}$, aperture $D=500 \mu \mathrm{m}$, and 
height $d_{1}=76 \mu \mathrm{m}$. The extra polymer layer we used to flatten the convex surfaces has thickness $d_{2}=81 \mu \mathrm{m}$. As a result, the microlens has a positive focal length $\left(f_{S}=4.26 \mathrm{~cm}\right)$ at $V=0$. As the applied voltage increases, the $\mathrm{LC}$ molecules are reoriented by the electric field. The voltage-induced refractive index change within the LC layer leads to a tunable focus microlens array. The resultant focal length of the microlens array can be positive or negative depending on the applied voltage.

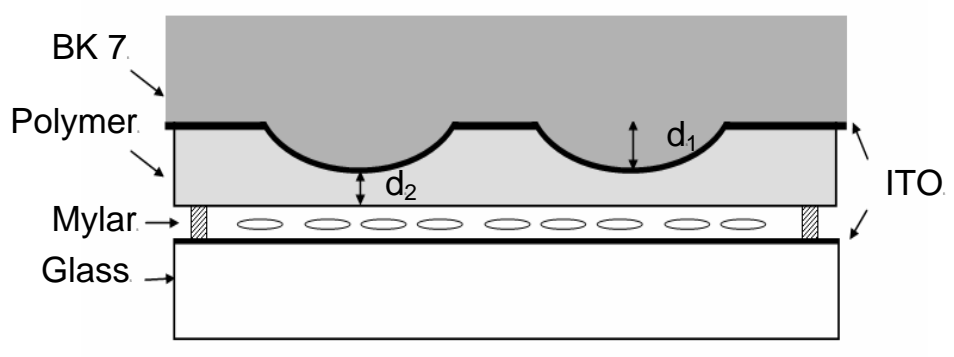

Figure 5.1: Schematic representation of the structure of a DFLC microlens array.

\section{$\underline{5.4 \text { Experimental Results and Discussions }}$}

A polarizing optical microscope was used to inspect the optical properties of the DFLC microlens array at different voltages. The rubbing direction of the cell was oriented at $45^{\circ}$ with respect to the axis of the linear polarizer and the analyzer was crossed to the polarizer. Figures 5.2(a)-(d) show the three photographs taken at $V=0,28$, 40 , and $200 \mathrm{~V}_{\mathrm{rms}}$, respectively.

At $V=0$, the sample is almost optically homogeneous; no interference rings were observed. As shown in Figure 5.2(a), no gradient refractive index distribution and nor focusing effect in the LC layer are found. The slight color difference is suspected due to 
the nonuniform cell gap which originates from the polymer shrinkage after polymerization process. When the applied voltage increases gradually, the LC molecules are reoriented starting from the center of each microlens and spreading over to the edges. This gradient phase retardation results in the interference rings observed in Figures 5.2(b) and (c). These rings are uniform and well formed indicating that the LC cell has good alignment and the disclination lines do not exist. Between the adjacent rings, the phase difference is $2 \pi$. At $40 \mathrm{~V}_{\mathrm{rms}}$, the maximum number of rings is obtained, which means the phase difference between the center and borders reaches the maximum. At this stage, the LC layer presents the shortest, but negative focal length. As the voltage is sufficiently higher than the threshold, most of the bulk LC directors are reoriented perpendicular to the substrates. Under such a circumstance, the refractive index profile is flattened and the focusing effect introduced by the LC layer is weaker, as shown in Figure 5.2(d). The focal length of the microlens becomes positive again. 
(a)

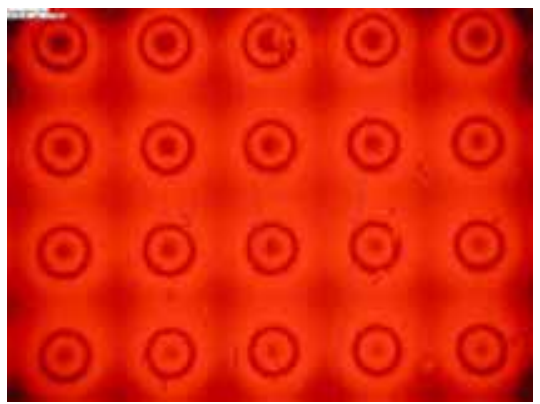

(b)

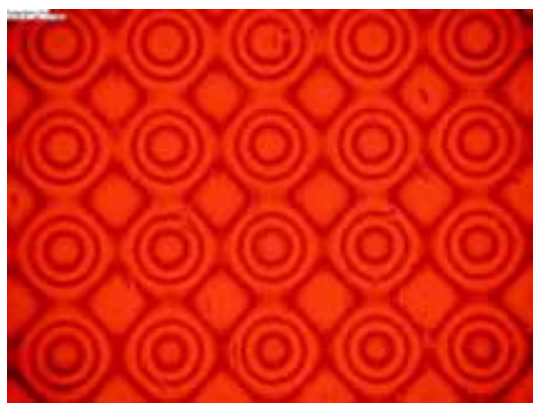

(c)

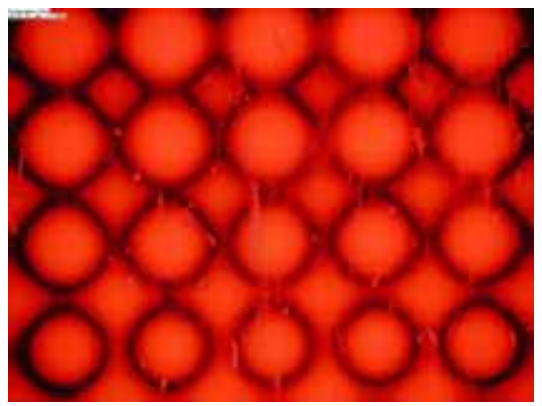

(d)

Figure 5.2: Microscope images of the DFLC microlens array at (a) $V=0$, (b) $28 \mathrm{~V}_{\text {rms }}$, (c) $40 \mathrm{~V}_{\text {rms }}$ and (d) $200 \mathrm{~V}_{\mathrm{rms}}$. The LC cell is sandwiched between crossed polarizers. The rubbing direction of the cell is oriented at $45^{\circ}$ with respect to the fast axis of the linear polarizer. 
Figure 5.3 shows the experimental setup for studying the focusing properties of the LC microlens array. The output beam of the He-Ne laser was magnified by two convex lenses with the focal lengths $50 \mathrm{~mm}$ and $250 \mathrm{~mm}$. A pinhole with $30 \mu \mathrm{m}$ diameter was put at the focal point of the first lens serving as a spatial filter. The beam diameter was expanded to $\sim 1 \mathrm{~cm}$. A sheet polarizer was used with its optical axis parallel to the cell rubbing direction. Light focusing properties of the cell was measured using a CCD camera (SBIG Model ST-2000XM) connected to a computer.

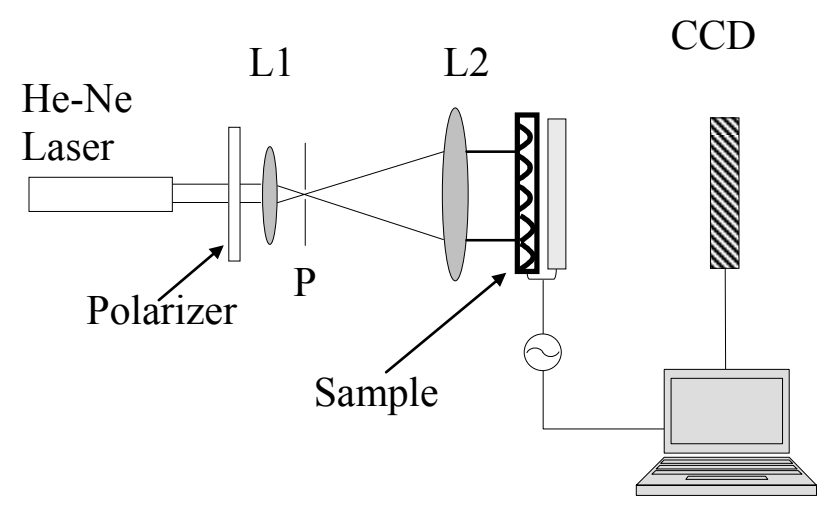

Figure 5.3: The experimental setup for studying the focusing properties of the microlens array. $\mathrm{L}_{1}$ : focal length $50 \mathrm{~mm}, \mathrm{~L}_{2}$ : focal length $250 \mathrm{~mm}$, and $\mathrm{P}: 30 \mu \mathrm{m}$ pinhole. The polarization of the incident light is parallel to the rubbing direction. 


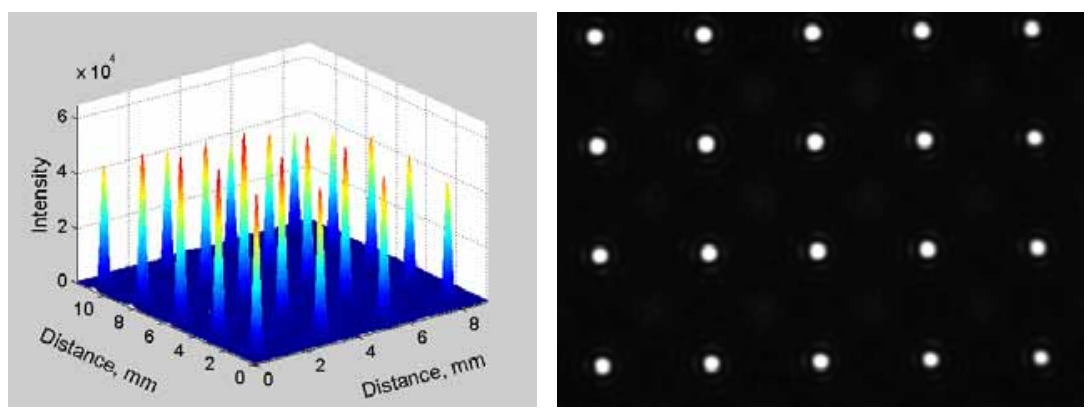

(a)

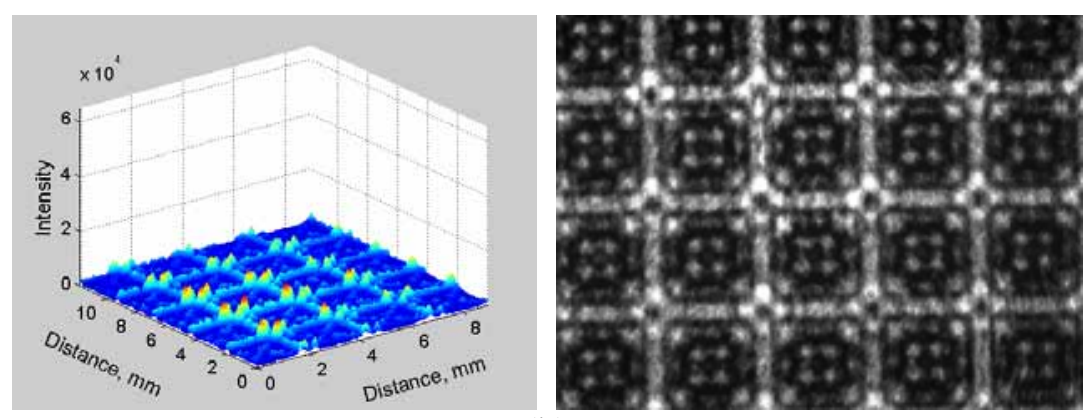

(b)
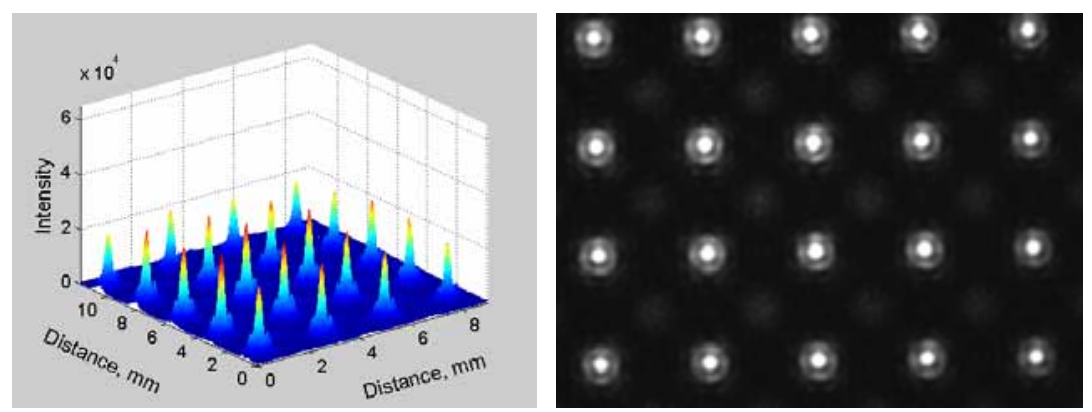

(c)

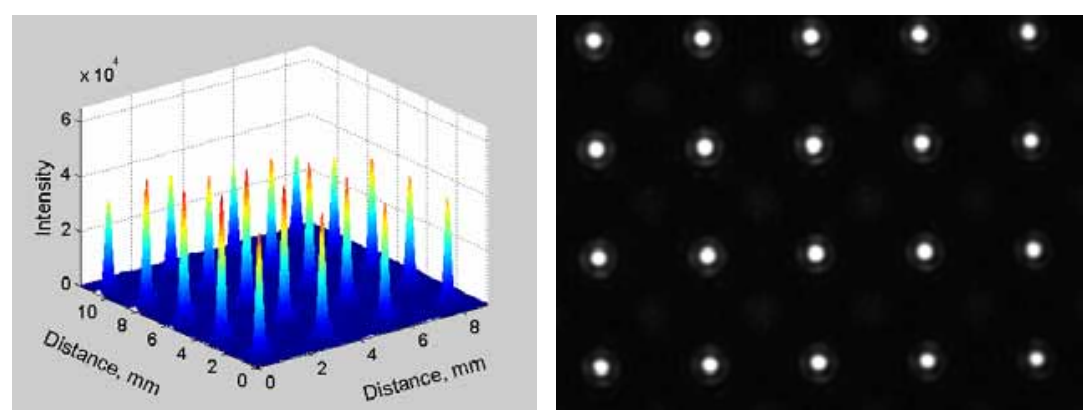

(d)

Figure 5.4: CCD images of the DFLC microlens array (left) and the corresponding 3-D light intensity profiles (right) at (a) 0 , (b) $50 \mathrm{~V}_{\mathrm{rms}}$, (c) $150 \mathrm{~V}_{\mathrm{rms}}$ and (d) $200 \mathrm{~V}_{\mathrm{rms}}$. 
The CCD was located at the initial focal plane of the microlens. The intensity profiles were measured at $V=0,50,150$ and $200 \mathrm{~V}_{\text {rms }}$, and results are shown in Figure 5.4. At $V=0$, the focusing effect is caused by the top substrate only, as show in Figure 5.4(a). When the applied voltage is increased, the LC layer adds the diverging focusing effect to the whole rooming lens system. As $V>30 \mathrm{~V}_{\mathrm{rms}}$, the focal length of the whole system becomes negative. At $50 \mathrm{~V}_{\mathrm{rms}}$, the beam is diverged to the edges of each single microlens, as shown in Fig. 5.4 (b). In the high voltage regime, the LC molecules are all perpendicular to the substrates. The gradient refractive index profile is gradually flattened and erased. The diverging effect of the LC lens is weakening. The microlens becomes converging lenses again, as shown in Figures 5.4 (c) and (d).

\section{$\underline{5.5 \text { Numerical Calculations and Simulations }}$}

To quantitatively explain this focal-length transition phenomenon, we calculate the voltage-induced LC director reorientation numerically. Using the same calculation methods we have stated in $\mathrm{Ch} 4$, we calculate the voltage-dependent refractive index change for a symmetric, uniform liquid crystal layer, and use this information to predict the voltage-dependent focal length.

Using the equations in $\mathrm{Ch} 4$ and parameters, as shown in Table 5.1, of our homemade dual-frequency liquid crystal (with pretilt angle, $\psi_{o}=2^{\circ}$ ), the voltagedependent average effective refractive index was calculated and plotted in Figure 5.5. Solid line and open circles represent the experimental and simulation results, respectively, at $\lambda=633 \mathrm{~nm}$. The agreement is very good. 
Table 5.1 The physical parameters of our homemade dual-frequency liquid crystal used for computer simulations

\begin{tabular}{|c|c|}
\hline Dual-frequency liquid crystal parameters & \\
\hline Effective splay elastic constants, $k_{11}$. & $33.5 \mathrm{pN}$ \\
Effective bend elastic constants, $k_{33}$. & $35 \mathrm{pN}$ \\
Parallel dielectric constant, $\varepsilon_{/ / .}$ & 12.17 \\
Perpendicular dielectric constant, $\varepsilon_{\perp}$ & 7.44 \\
Ordinary refractive index, $n_{\mathrm{o}}$ & 1.49 \\
Extraordinary refractive index, $n_{\mathrm{e}}$ & 1.775 \\
\hline
\end{tabular}

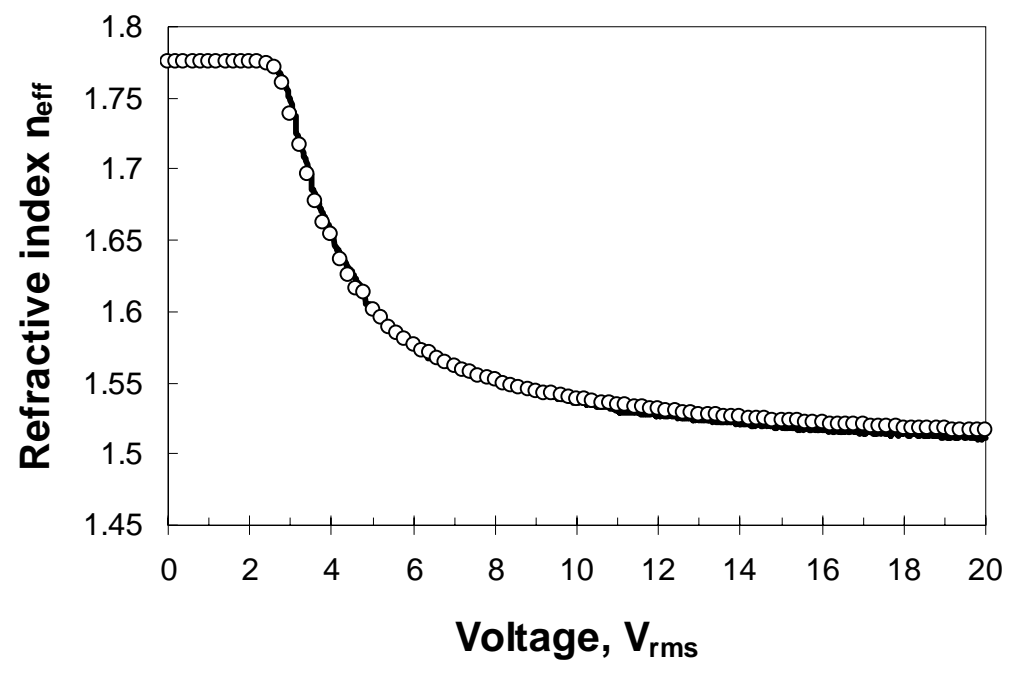

Figure 5.5: Voltage-dependent average refractive index of dual-frequency liquid crystal used in the experiment. Solid line and open circles represent the experimental and simulation results, respectively. $\lambda=633 \mathrm{~nm}$. 
When a voltage $\mathrm{V}$ is applied to the LC mircolens array, the electric field in the center $\left(E_{\text {Center }}\right)$ and at the border $\left(E_{\text {Border }}\right)$ of each microlens is expressed as: $:^{90}$

$$
\begin{gathered}
E_{\text {center }}=\frac{V / \varepsilon_{L C}}{\frac{d_{L C}}{\varepsilon_{L C}}+\frac{d_{2}}{\varepsilon_{p}},} \\
E_{\text {border }}=\frac{V / \varepsilon_{L C}}{\frac{d_{L C}}{\varepsilon_{L C}}+\frac{d_{1}+d_{2}}{\varepsilon_{p}}},
\end{gathered}
$$

where $d_{1}$. represents the microlens thickness and $d_{2}$ is the thickness of the extra polymer material shown in Figure 5.1, $d_{L C}$ is the LC layer thickness, and $\varepsilon_{\mathrm{LC}}$ and $\varepsilon_{\mathrm{p}}$ represent the dielectric constant of the LC and polymer, respectively. At $V=0, \varepsilon_{L C}=\varepsilon_{\perp}$. As $V>V_{t h}$, the effective $\varepsilon_{\mathrm{LC}}$. will change and affect the electrical field distribution through the cell. Finally, the equilibrium state is achieved. By using the final voltage distribution, we can calculate the LC tilt angle, average effective birefringence, and the phase difference between the center and borders of each microlens.

The focal length of an LC lens can be calculated from Fresnel's approximation:

$$
f=\frac{r^{2}}{2 \delta n d_{L C}},
$$

where $r=D / 2$ ( $D$ is the microlens diameter) and $\delta n=\widetilde{n}_{c e n t e r}-\widetilde{n}_{b o r d e r}$ is the refractive index difference between the lens center and borders.

To measure the focal length we focused the CCD camera on the microlens surface and then moved the microlens arrays toward the laser source until a sharp focal point was found. The distance that the sample traveled was equal to its focal length. ${ }^{91}$. 


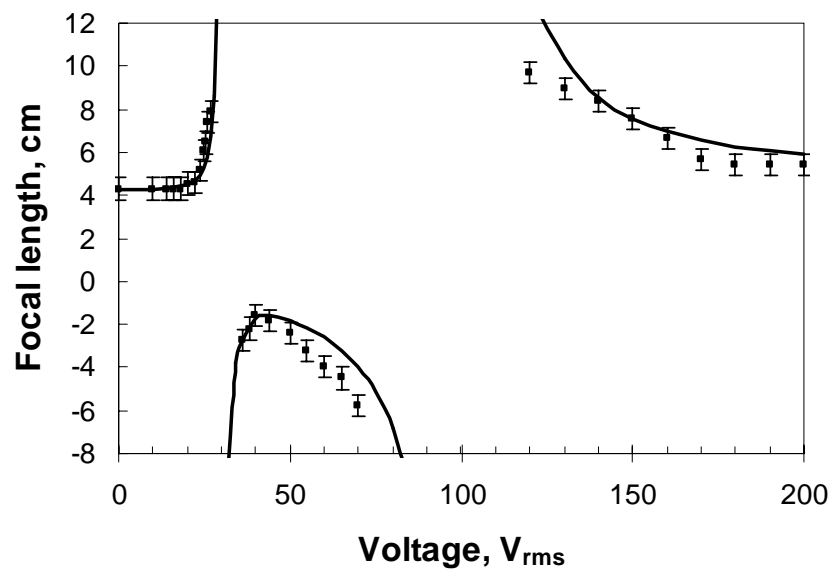

Figure 5.6: Voltage-dependent focal length of the DFLC microlens array. LC cell gap $d=$ $18 \mu \mathrm{m}$; the diameter of microlens $D=500 \mu \mathrm{m} ; \lambda=633 \mathrm{~nm}$.

The voltage-dependent focal length of the lens was investigated and compared with the simulation results, as shown in Figure 5.6. At $V=0, \mathrm{LC}$ directors are aligned homogeneously and no focusing effect occurs in LC layer. The system shows the initial focus contributed solely by the top substrate. As the voltage increases, the focal length is increased accordingly. At $V \sim 30 \mathrm{~V}_{\text {rms }}$, the microlens starts changing to become a diverging lens. At $\sim 40 \mathrm{~V}_{\mathrm{rms}}$, as we observed in the microscopic photos shown in Figure 5.2, the microlens reached the shortest negative focal length. Further increasing the voltage would reorient all the $\mathrm{LC}$ molecules perpendicular to the substrates and reduce the phase difference. The microlens becomes converging lens again and gradually approaches the initial focal length but at a different rate. The simulation results agree with experiment quite well in the lower voltage regime $\left(<40 \mathrm{~V}_{\mathrm{rms}}\right)$ but in the higher voltage regime (between 40 and $130 \mathrm{~V}_{\mathrm{rms}}$ ) the fitting is somewhat deviated. This is because in the simulations we assume the anchoring energy at the boundaries is infinity, which means 
that the LC molecules near the substrates will not be reoriented at all by the electric field. In our experiment, the LC molecules near the substrates would still rotate at a high voltage. Therefore, in the high voltage regime the measured focus change is faster than that simulated. Above $140 \mathrm{~V}_{\text {rms }}$, the LC molecules are reoriented nearly perpendicular to the substrates in both experimental data and simulation results. Therefore, the measured focal length agrees well with the simulated values in the high voltage regime.

\subsection{Driving Scheme and Response Time}

The response time of the lens was measured by switching the lens from converging to diverging, i.e., from 0 to $50 \mathrm{~V}_{\text {rms }}$. The rise time and decay time is 615 and $617 \mathrm{~ms}$, respectively. The slow response time is the direct consequence of the thick LC layer $(18-\mu \mathrm{m})$ employed. The rise time can be reduced by using the overdrive technique. ${ }^{92}$. If we drive the microlens array with $f=1 \mathrm{kHz}, 200 \mathrm{~V}_{\text {rms }}$ for $20 \mathrm{~ms}$ and then followed by a $50 \mathrm{~V}_{\mathrm{rms}}$. holding voltage, the rise time is reduced to $3.87 \mathrm{~ms}$, as shown in Figure 5.7. To improve decay time, we take the advantage of the dual-frequency LC whose dielectric anisotropy turns to negative at a high frequency. The DFLC we employed has $\Delta \varepsilon=-3.93$ at $50 \mathrm{kHz}$. When we applied $50 \mathrm{~V}_{\mathrm{rms}}$ and $f=1 \mathrm{kHz}$ to turn the microlens to diverging lens and switched it back by applying a high frequency $(f=50$ $\mathrm{kHz}$ ) voltage burst $\left(V=200 \mathrm{~V}_{\mathrm{rms}}\right)$, the decay time is reduced to $5.37 \mathrm{~ms}$ as depicted in Fig. 5.7. The operating voltage can be further reduced if we reduce the $d_{2}$ thickness and increase the dielectric constant of the filling polymer. 


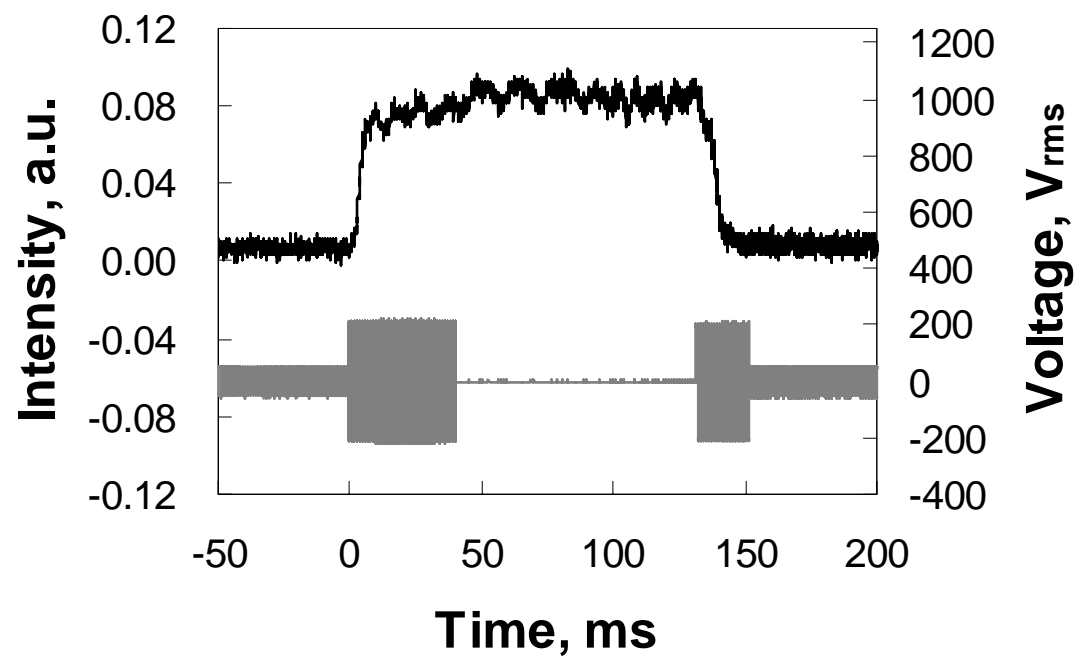

Figure 5.7: The oscilloscope traces of the voltage waveforms (lower trace) and the corresponding optical responses (upper trace) of the DFLC microlens array. The measured rise and decay time is 3.87 and $5.37 \mathrm{~ms}$, respectively.

\subsection{Conclusion}

A flat microlens array whose focal length can be switched from positive to negative by electric field is demonstrated experimentally and confirmed by computer simulations. To generate the required gradient refractive index, an inhomogeneous electric field is created by a spherical indium-tin-oxide (ITO) electrode which is imbedded in the top flat substrate. The bottom substrate has a planar ITO electrode on its inner surface. A thin polymeric layer is overcoated on top of the spherical ITO to create flat surface. The disclination lines are eliminated. Because of the employed dualfrequency liquid crystal, the microlens array has fast response times.

In conclusion, we have demonstrated an electrically tunable-focus DFLC microlens array using the combination of spherical and planar electrodes. The microlens 
array possesses diverging and converging focusing behaviors depending on the applied voltage. These focal length transition phenomena are confirmed by computer simulations. Using the two frequency driving scheme, the lens response time is about $5 \mathrm{~ms}$. 


\section{CHAPTER 6: ANISOTROPIC AND ISOTROPIC LIQUID-CRYSTAL GELS}

\section{$\underline{6.1 \text { Introduction }}$}

Liquid-crystal gel $^{32,93}$ is also known as polymer-stabilized liquid crystal (PSLC) or polymer network liquid crystal (PNLC). It exhibits an anisotropic light scattering which is different from the polymer-dispersed LC. To form an LC gel, a few percent (3-8 wt \%) of diacrylate monomer is mixed in a nematic LC host. The mixture is injected into an empty LC cell with surface rubbing and then exposed to a weak ultraviolet light.

For a reversed-mode LC gel, ${ }^{28}$ the gel is highly transparent in the absence of an electric field. As the voltage exceeds a threshold, the polymer networks and the electric field are working against each other resulting in multi-domain structures that scatter the light with polarization parallel to the rubbing direction. The polarization which is perpendicular to the rubbing direction is transmitted. For a homogeneous alignment gel using a positive dielectric anisotropy ( $\Delta \varepsilon>0$ ) LC, the contrast ratio could exceed 2000:1, depending on the curing conditions. ${ }^{27}$. The device is polarization sensitive. For a homeotropic alignment gel employing a negative $\Delta \varepsilon \mathrm{LC}$, the contrast ratio is only $\sim 10: 1$ for an unpolarized light. ${ }^{93}$ The response time of both gels is $\sim 10 \mathrm{~ms}$, depending on the LC material employed and the polymer concentration. 
Such an LC gel can be used for reflective display, broadband switchable polarizer, light shutter, and variable optical attenuators for telecommunication. ${ }^{11,26-28,31,32,93,94}$. By reducing the multi-domain size, light scattering could be eliminated in the near infrared spectral region. Such a LC polymer network exhibits a fast response time except for the increased operating voltage. ${ }^{95}$

\section{$\underline{\text { 6.2 Normal-Mode Anisotropic Liquid-Crystal Gels }}$}

\subsubsection{Experimental Methods}

In this work, we demonstrate an experimental method for preparing a normalmode anisotropic LC gel and explore its electro-optic properties. The term "normal mode" refers to that the gel is in the scattering state at $V=0$ and is highly transparent in a high-voltage state. For display applications, the normal mode LC gel when doped with a black dye leads to a normally black display.

In our studies, the LC gel was fabricated by photopolymerizing a nematic LC host (Merck E48, $\Delta n=0.231$ ) and photocurable monomer. In the reverse mode, the monomer concentration $(c)$ is typically in the $3-10 \mathrm{wt} \%$ range. However, to obtain a normal mode, the monomer concentration is below $1 \%$ and an electric field needs to be applied during polymerization process. Four samples with $c=0.4,0.6,0.8$, and $1.0 \mathrm{wt} \%$ were prepared. The monomer used in this study is bisphenol-A-dimethacrylate with structure shown as follows: 


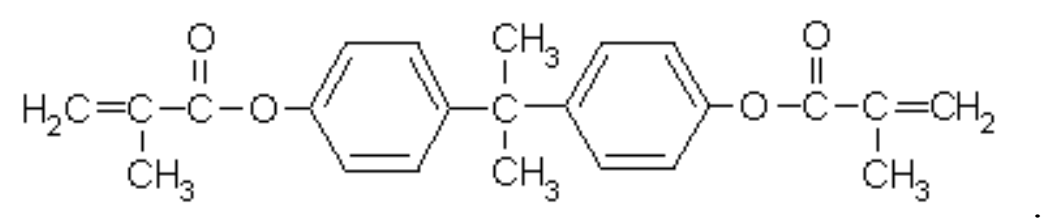

The $\mathrm{LC} /$ monomer mixture was filled in the cells with gap $d=10 \mu \mathrm{m}$. The inner surfaces of the indium-tin-oxide glass substrates were overcoated with a thin polyimide layer and buffed in anti-parallel directions for achieving homogeneous alignment. The cell was exposed to UV light under $10 \mathrm{~V}_{\text {rms }}$ biased voltage such that the $\mathrm{LC}$ directors were oriented to the electric-field direction and the monomers were polymerized along the same direction which is perpendicular to the substrates. To avoid incomplete polymerization, a weak UV intensity $\left(\mathrm{I} \sim 30 \mathrm{~mW} / \mathrm{cm}^{2}\right)$ was used. The exposure time was 3 hours. The resulting polymer network was perpendicular to the substrate surface. Weak UV exposure and a slow polymerization process were found to be crucial to achieve a better dark state, higher contrast ratio, and lower operating voltage. Slow polymerization coarsened the polymer network, stabilized the polymer network, and enlarged the LC domains. ${ }^{96}$

\subsubsection{Experimental Results and Discussions}

The electro-optic properties of the LC gels were studied by measuring the transmittance of the LC gels with a He-Ne laser. The laser beam diameter is about $1 \mathrm{~mm}$. A photodiode detector was set at $\sim 30 \mathrm{~cm}$ away from the LC sample. A sheet polarizer with its optical axis parallel to the rubbing direction of the substrate was put in front of 
the LC cell. The voltage-dependent transmittance of the LC gels is shown in Figure 6.1 for $c=0.4,0.6,0.8$, and $1.0 \mathrm{wt} \%$ and $d=10 \mu \mathrm{m}$.

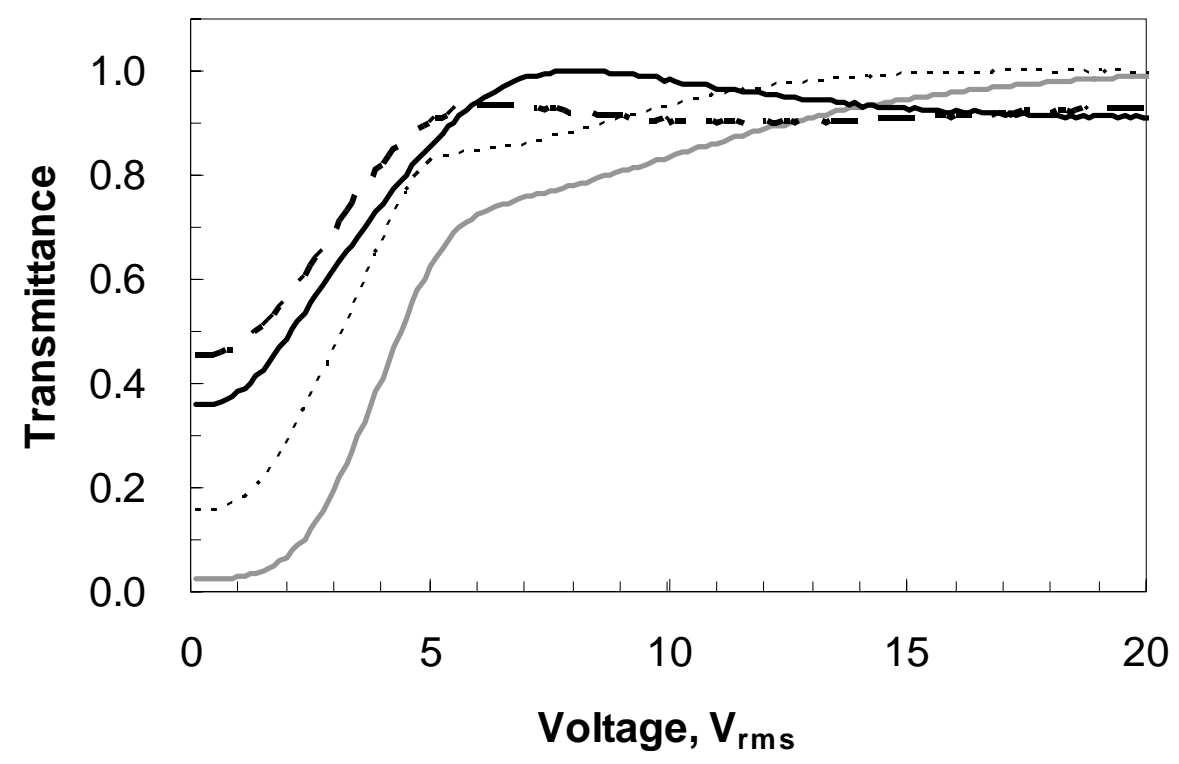

Figure 6.1: Voltage-dependent normalized transmittance of the LC gels at different monomer concentrations in a $10 \mu \mathrm{m}$ cell. From top to bottom, $c=1.0,0.8,0.6$, and $0.4 \mathrm{wt}$ $\%$.

Figure 6.1 shows the voltage-dependent normalized transmittance of the $\mathrm{He}-\mathrm{Ne}$ laser beam polarized in the rubbing direction. The absolute transmittance of the on state reaches $98 \%$ if the multiple reflections of the substrates are excluded. Figure 6.2 shows the schematic diagrams illustrating the molecular arrangements of the voltage-off and -on states of the normal-mode LC gel. At $V=0$, the buffed polyimide layers tend to align LC molecules parallel to the rubbing direction, but the polymer networks tend to retain the LC directors perpendicular to the substrate. As a result, multiple domains are formed. The 
light polarized along the rubbing direction is scattered while the perpendicular component is transmitted without scattering.

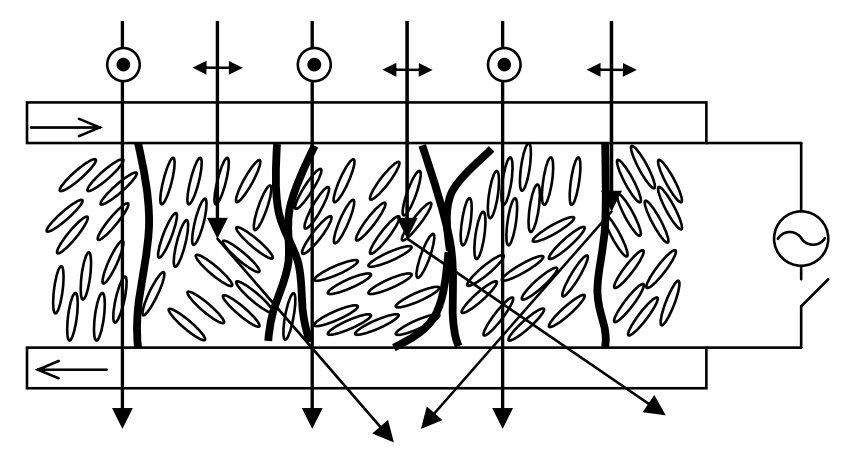

(a) Voltage off

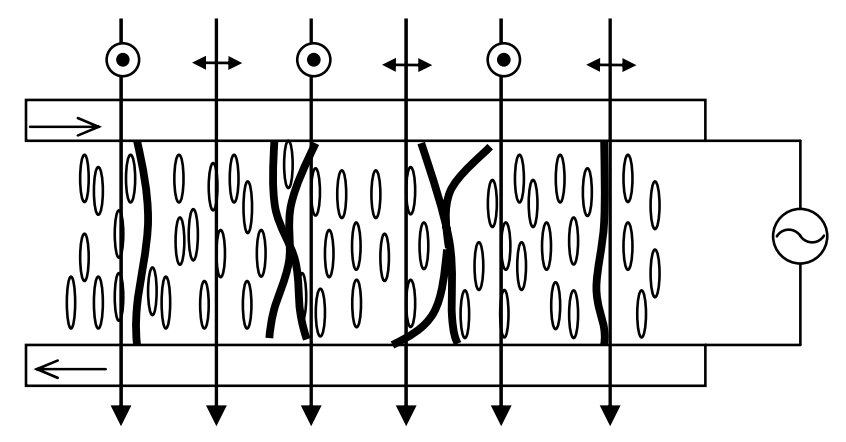

(b) Voltage on

Figure 6.2: Schematic representation of the operating principle of LC gels. (a) Voltageoff state and (b) voltage-on state. The arrows in the substrates indicate the rubbing directions.

From Figure 6.1, monomer concentration plays an important role in determining the dark state of the normal-mode LC gel. The lower monomer concentration leads to a better dark state. For a higher monomer concentration, the anchoring force of the polymer network is stronger so that more LC molecules are aligned perpendicular to the substrates. Under such a circumstance, the gel is more ordered and its light scattering capability is 
weaker. As the monomer concentration is reduced to a certain level, the network anchoring force is comparable to the polyimide-induced alignment force. As a result, the gel is highly disordered and light scattering dominates in the voltage-off state. From Figure 6.1 , the measured contrast ratio is $\sim 100: 1$ for the sample with $0.4 \%$ monomer concentration. If we stack two anisotropic gels in orthogonal directions, the stacked cells transmit the input unpolarized light also with high efficiency. When one of the cells is activated, the output light becomes linearly polarized. When both cells are activated, both polarizations are scattered and the device functions like crossed polarizers. An extinction ratio greater than 150:1 was measured using two $c=0.4 \%$ LC gels and an unpolarized $\mathrm{He}-\mathrm{Ne}$ laser.

Figure 6.3 shows the measured transmission spectra of the $d=10 \mu \mathrm{m} \mathrm{LC} \mathrm{gel} \mathrm{in}$ the voltage-off and -on states. The on-state voltage is $10 \mathrm{~V}_{\mathrm{rms}}$. The spectral bandwidth covers the whole visible region. Such an anisotropic LC gel can be used as a white-light switchable polarizer. In the voltage-off state, the gel scatters the polarization along the buffing direction so that the transmitted light becomes linearly polarized. In the voltageon state, the gel is highly transparent and is independent of polarization.

We also studied the UV intensity effect on the gel formation. Figure 6.4 compares the voltage-dependent transmittance of the LC gels with same monomer concentration ( $c$ $=0.4 \%$ ) but prepared at two different UV intensities: 30 and $100 \mu \mathrm{w} / \mathrm{cm}^{2}$. For the weaker UV exposure, the polymerization process is slower leading to a coarser polymer network and lower operating voltage. Under such a circumstance, the polymerization process is more complete and the polymer network is more stable resulting in a better dark state. 


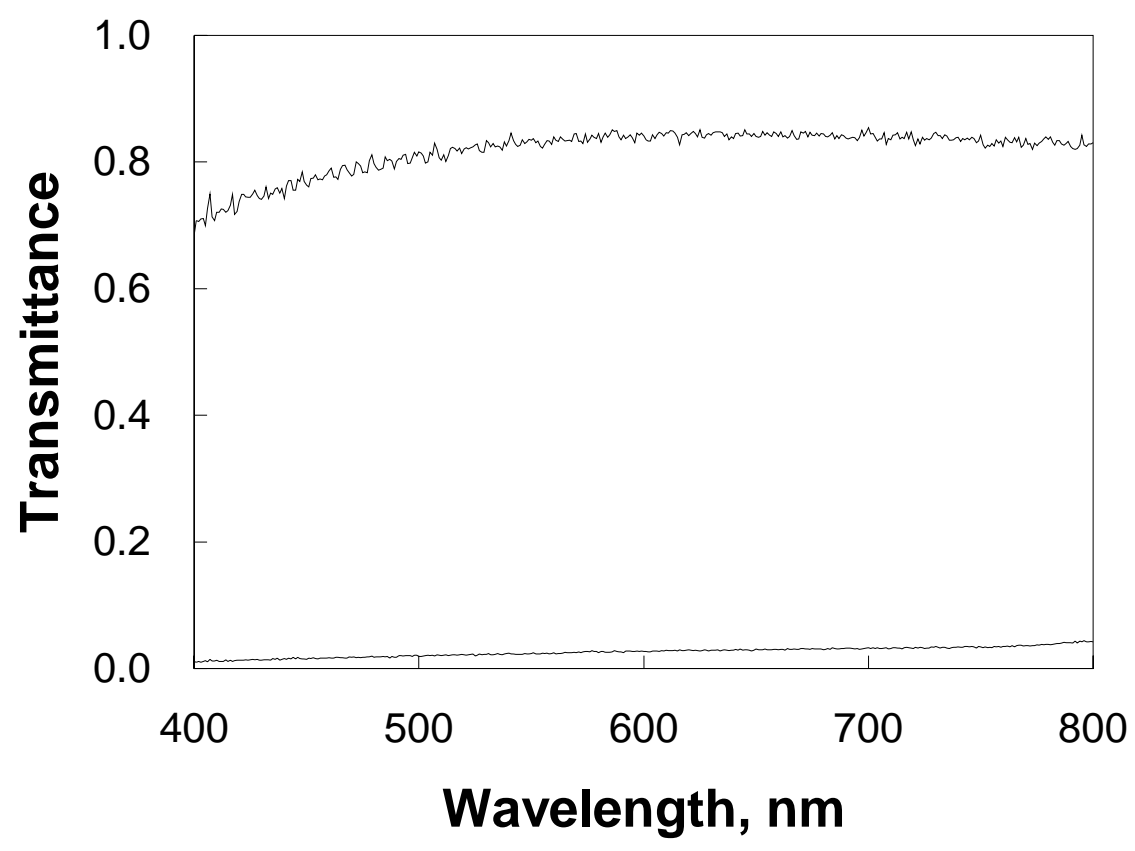

Figure 6.3: The spectral bandwidth of a $10 \mu \mathrm{m} \mathrm{LC}$ gel. Upper trace: $V=10 \mathrm{~V}_{\mathrm{rms}}$; lower trace: $V=0$. A calcite polarizer was used to polarize the incident light.

The rise and fall time of the $c=0.4 \%$ and $d=10 \mu \mathrm{m} \mathrm{LC}$ gel was measured to be 1.5 and $192 \mathrm{~ms}$, respectively. To reduce the fall time, we could increase the monomer concentration or decrease the cell gap. Increasing monomer concentration would lead to a stronger anchoring force between the polymer network and LC interfaces. Therefore, the threshold voltage is increased but the decay time is decreased. On the other hand, decreasing the cell gap would substantially reduce the decay time except that the contrast ratio is also decreased. To boost the contrast ratio, a high birefringence LC mixture should be employed. 


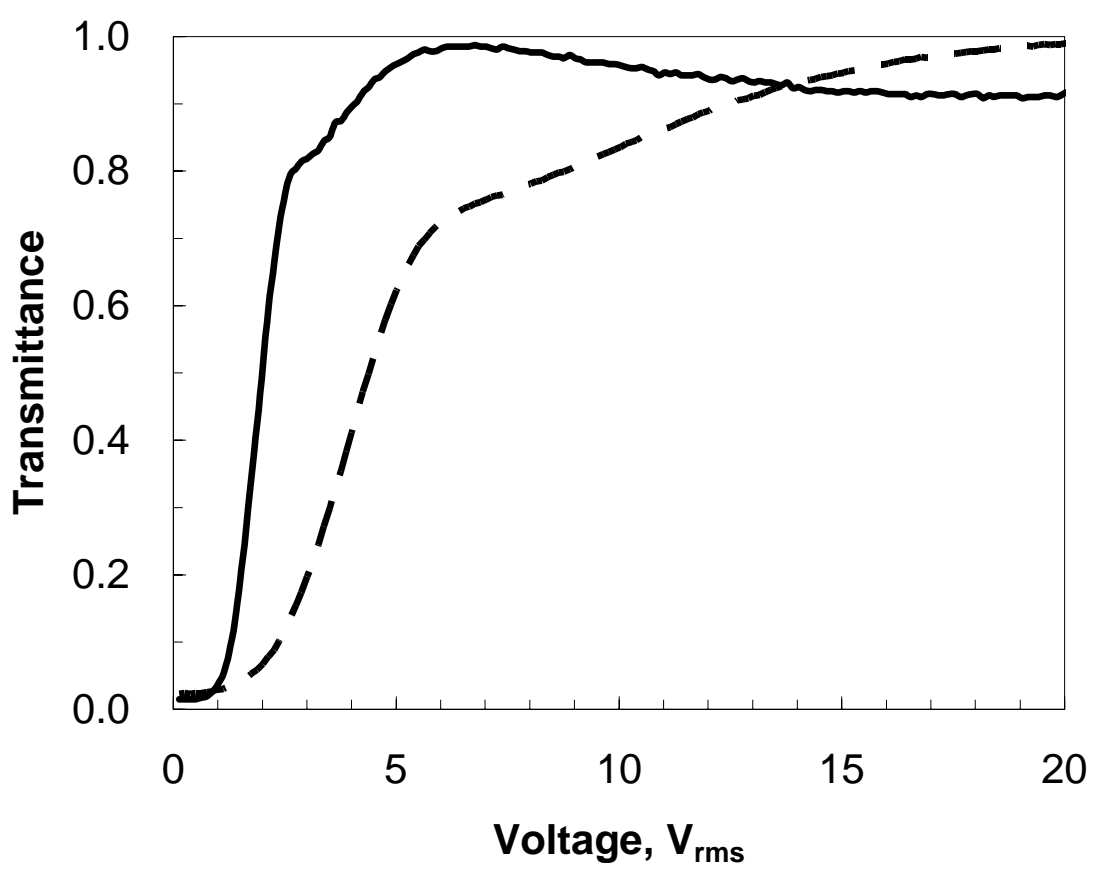

Figure 6.4: Voltage-dependent normalized transmittance of the LC gels with monomer concentration of 0.4 wt. $\%$ at 30 (solid lines) and 100 (dashed lines) $\mu \mathrm{W} / \mathrm{cm}^{2}$. UV intensities.

We have applied the LC gel to reflective display. Figure 6.5 shows the device configuration which consists of an anisotropic gel cell, a broadband quarter-wave film, and a reflector. The angle between the optical axis of the wave plate and cell rubbing direction is $45^{\circ}$. In the voltage-off state, the input polarization ( $p$-wave) which is parallel to the cell rubbing direction is highly scattered at the first pass. The transmitted $s$-wave traverses the $\lambda / 4$ film twice and is converted to $p$ wave. During the return path, this $p$ wave is again scattered resulting in a translucent state. In the voltage-on state, the cell does not affect the incident polarization at all. The incident light traverses through the cell and wave plate and is reflected back by the reflector with high efficiency. The 
contrast ratio (CR) of the $c=0.4 \%$ and $d=10 \mu \mathrm{m} \mathrm{LC}$ gel was measured to be $\sim 60: 1$. For the $c=0.6 \%$ and $d=8 \mu \mathrm{m} \mathrm{LC} \mathrm{gel,} \mathrm{we} \mathrm{obtained} \mathrm{CR} \sim 40: 1$, rise time $\sim 2 \mathrm{~ms}$, and fall time $\sim 65 \mathrm{~ms}$. To reduce response time further while retaining a good contrast ratio, a high birefringence LC mixture could be used and cell gap reduced.

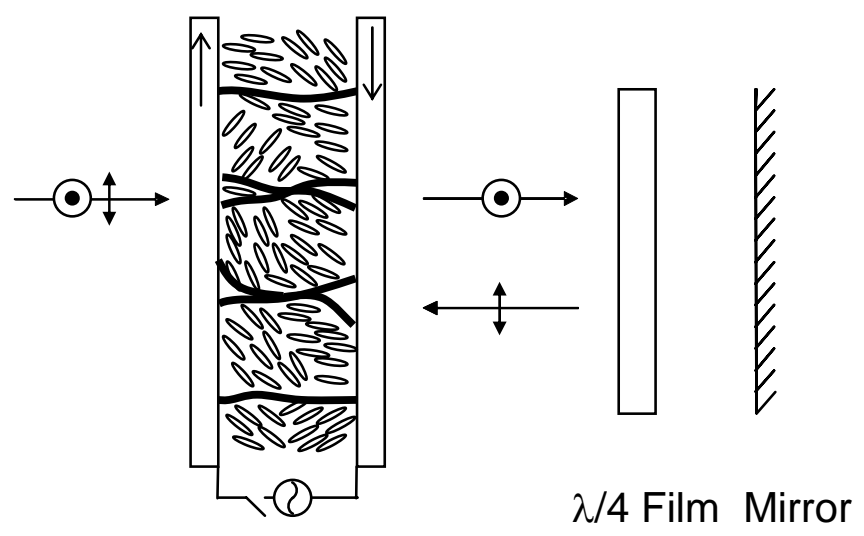

(a) Voltage-Off

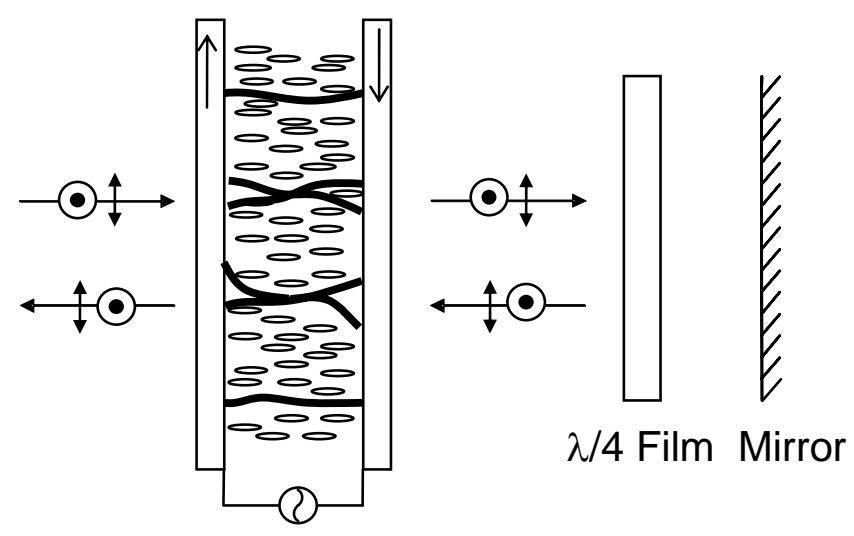

(b) Voltage-On

Figure 6.5: A reflective display consists of a normal-mode LC gel, a quarter-wave film, and a reflector. (a) Voltage-off state and (b) voltage-on state. The arrows in the substrates indicate the rubbing directions. 


\subsubsection{Conclusion}

A normal-mode anisotropic liquid crystal (LC) gel is demonstrated. In the absence of an electric field, the light polarized along the rubbing direction is scattered. In the voltage-on state, the gel is highly transparent and is independent of polarization. Such a normal mode LC gel exhibits a modest contrast ratio, response time, and low operating voltage. It can be used as a broadband switchable polarizer, variable optical attenuator, and reflective display.

In conclusion, a normal-mode LC gel is demonstrated. Such a device exhibits a modest contrast ratio and low operating voltage. The potential applications of LC gel can be found in switchable polarizer, telecom optical switch, and reflective displays.

\subsection{Dual-Frequency Liquid Crystal Gels with Submillisecond Response Time}

\subsubsection{Experimental Methods}

In this work, we demonstrate fast response time and high contrast ratio gels using

a dual-frequency liquid crystal (DFLC). ${ }^{86}$. For a homogeneous gel, the light scattering is anisotropic. However, for a DFLC gel prepared using substrates without alignment layer, the light scattering is independent of polarization and the response time is $\sim 0.8 \mathrm{~ms}$.

The LC gels were fabricated by photopolymerizing the DFLC/monomer mixture. The DFLC mixture we employed consists of some biphenyl esters and lateral difluoro tolanes. Its physical properties are summarized as follows: cross-over frequency $f_{c} \sim 5$ 
$\mathrm{kHz}$, birefringence $\Delta n \sim 0.277$ (at $\lambda=633 \mathrm{~nm}$ and $T=22{ }^{\circ} \mathrm{C}$ ), and dielectric anisotropy $\Delta \varepsilon=6.6$ at $f=1 \mathrm{kHz}$ and $\Delta \varepsilon=-4.1$ at $50 \mathrm{kHz}$. Two samples with 5 and $7 \mathrm{wt} \%$ monomer concentration were prepared. The photocurable monomer used in this study is bisphenol A dimethacrylate. ${ }^{97}$. The $\mathrm{LC} /$ monomer mixture was filled in the cells with gap $d=8 \mu \mathrm{m}$. In our experiment, two types of LC cells were studied: one has homogeneous alignment and the other has no polyimide alignment layer. For the homogeneous cell, the inner surfaces of the indium-tin-oxide (ITO) glass substrates were overcoated with a thin polyimide layer and buffed in anti-parallel directions. In both cells, the LC/monomer mixture was irradiated by a UV light $(\lambda \sim 365 \mathrm{~nm})$ under the application of a bias voltage $\left(V_{b}=15 \mathrm{~V}_{\mathrm{rms}}\right)$ across the cell so that the LC and monomer molecules were oriented at nearly perpendicular to the substrates. As a result, the formed chain-like polymer networks are along the electric field direction. The UV curing intensity was $I \sim 20$ $\mathrm{mW} / \mathrm{cm}^{2}$ and curing time $\sim 10 \mathrm{~min}$.

We measured the transmittance of both LC gels using a He-Ne laser beam at normal incidence angle. The laser beam diameter is $\sim 1 \mathrm{~mm}$. A photodiode detector was set at $\sim 25 \mathrm{~cm}$ away from the LC sample, which corresponds to $\pm 1^{\circ}$ acceptance angle. A linearly polarized $\mathrm{He}-\mathrm{Ne}$ laser was used for studying the anisotropic scattering behavior of the homogeneous gel and an unpolarized He-Ne laser was used to characterize the isotropic DFLC gel. 


\subsubsection{Experimental Results and Discussions}

Figure 6.6 shows the voltage-dependent transmittance of the $8-\mu \mathrm{m}$ homogeneous LC gels at two monomer concentrations $(c), c=5$ and $7 \mathrm{wt} \%$. The $100 \%$ transmittance in Figure 6.6 represents the laser transmission of an $8-\mu \mathrm{m}$ homogeneous cell filled with the DFLC mixture alone. In the gel experiment, the laser polarization is parallel to the LC rubbing direction. In the voltage-off state, the polymer networks align LC molecules perpendicular to the substrates, similar to a homeotropic LC cell shown in Figure 6.7(a). When a low-frequency $(f=1 \mathrm{kHz})$ electric field is applied to the cell, the LC molecules are aligned more orderly (perpendicular to the substrates). As a result, the transmission is slightly increased, as shown by the gray line in Figure 6.6. If a high frequency $(f=50$ $\mathrm{kHz}$ ) voltage is applied, the DFLC exhibits a negative dielectric anisotropy. The cell is equivalent to a negative LC in a homeotropic cell. As the applied voltage exceeds a threshold $\left(\sim 12 \mathrm{~V}_{\text {rms }}\right.$ for the $c=5 \mathrm{wt} \%$ gel $)$, the LC molecules are switched into a multidomain structure, as illustrated in Figure 6.7(b). Thus, light scattering takes place and transmittance begins to decrease. The LC directors in different domains have different orientations. The resultant refractive index mismatch scatters light at the interfaces between domains. Due to the anti-parallel alignment layers on the substrates, the LC molecules will be reoriented in the $x-z$ plane. For the light with polarization perpendicular to the rubbing direction, it encounters the ordinary refractive index $n_{O}$ in both voltage-off and -on states. This ordinary ray passes through the cell without being scattered. On the

other hand, the light polarized along the rubbing direction is scattered. The measured contrast ratio is $\sim 360: 1$ for the $c=5 \mathrm{wt} \%$ sample. 


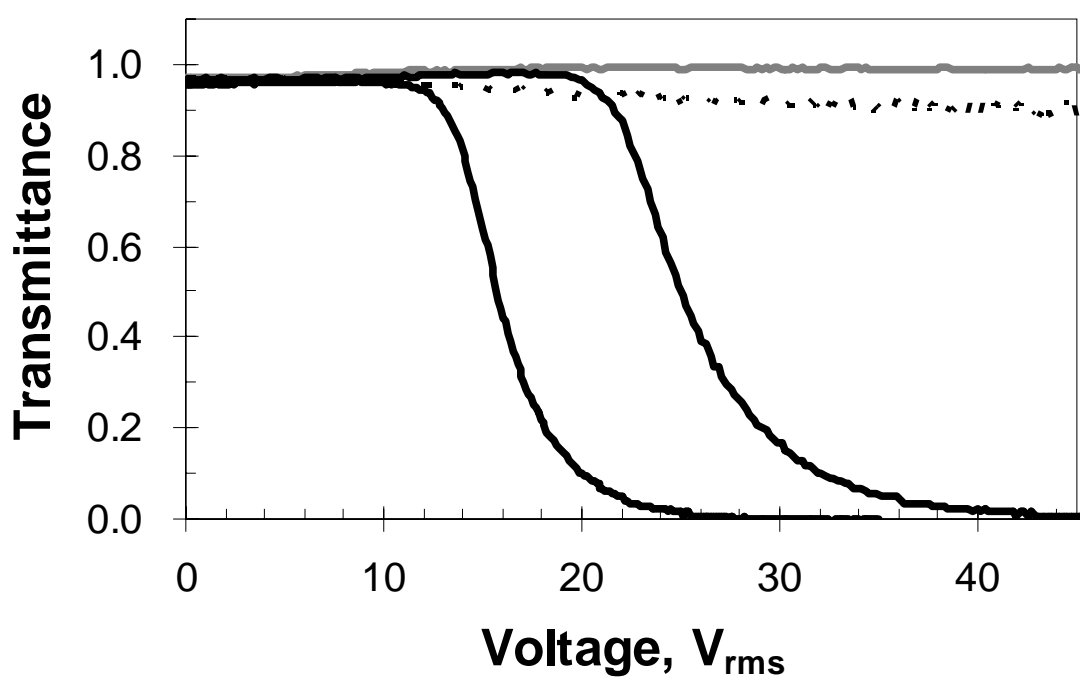

Figure 6.6: Voltage-dependent normalized transmittance of the homogeneous-aligned DFLC gels at different monomer concentrations. Solid and gray lines are for $f=50 \mathrm{kHz}$ and $1 \mathrm{kHz}$, respectively, and polarization $\|$ to the cell rubbing direction. Dashed lines are for $c=5 \mathrm{wt} \%$ and $f=50 \mathrm{kHz}$, but polarization ${ }^{\wedge}$ to the cell rubbing direction. Cell gap $d$ $=8 \mu \mathrm{m}, \lambda=633 \mathrm{~nm}$, and $T=22{ }^{\circ} \mathrm{C}$.

To measure the response time of the DFLC gel, we could switch voltage at a high frequency or switch frequency at a fixed voltage. In the case of switching voltage between 0 and $30 \mathrm{~V}_{\text {rms }}(f=50 \mathrm{kHz})$, the rise time was measured to be $2 \mathrm{~ms}$ and decay time $21 \mathrm{~ms}$. The faster rise time is due to the voltage assisted molecular reorientation. ${ }^{94}$. If we fix the voltage at $30 \mathrm{~V}_{\text {rms }}$ while switching frequency between $1 \mathrm{kHz}$ and $50 \mathrm{kHz}$, the rise and decay time is reduced to $2 \mathrm{~ms}$ and $5.5 \mathrm{~ms}$, respectively. At the translucent state, the gel scatters the light polarized along the buffing direction so that the transmitted light remains linearly polarized. Such an anisotropic LC gel can be used as a white light switchable polarizer or reflective display. ${ }^{28}$ 


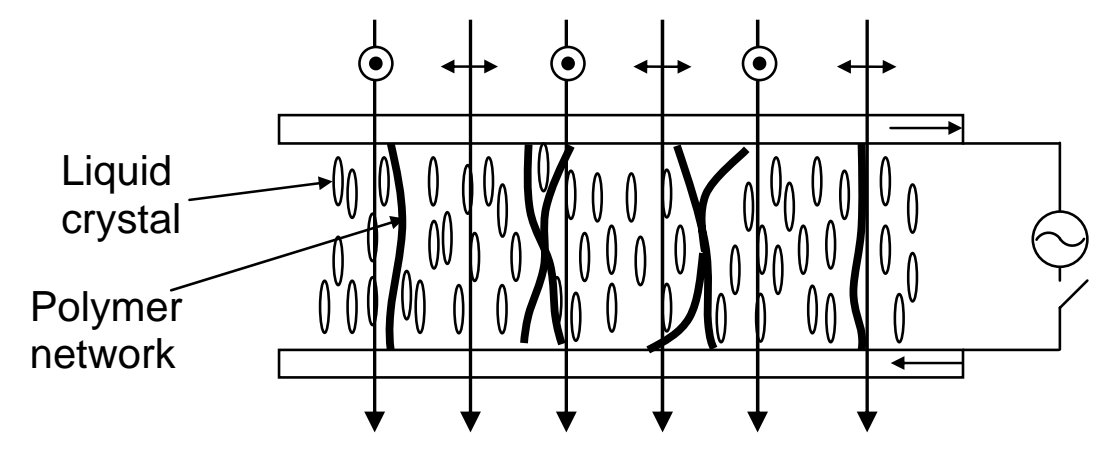

(a) Voltage-off

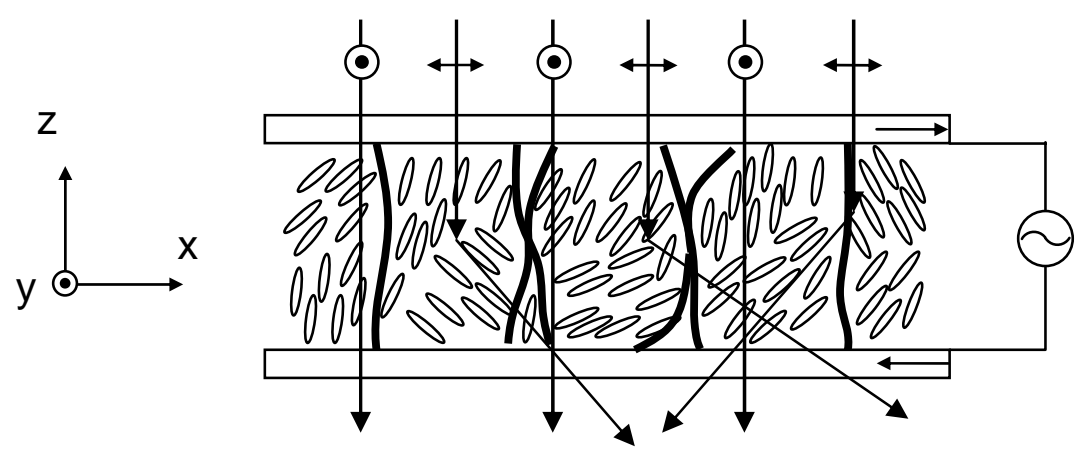

(b) Voltage-on $(50 \mathrm{kHz})$

Figure 6.7: Schematic representation of the operating principle of homogeneous-aligned DFLC gels. (a) Voltage-off state and (b) voltage-on state. The arrows in the substrates indicate the rubbing directions.

The polymer concentration plays a very important role affecting the contrast ratio and gel stability. A too small monomer concentration results in lower density polymer networks and weaker polymer anchoring force, which makes the polymer network unable to hold the LC molecules perpendicular to the substrates. In our experiment, we found if the monomer concentration is below $5 \mathrm{wt} \%$, the gel becomes irreversible after having 
been switched for several cycles. On the other hand, a high monomer concentration leads to an increased threshold voltage. From Figure 6.6, the threshold voltage of the $7 \% \mathrm{LC}$ gel is $\sim 20 \mathrm{~V}_{\mathrm{rms}}$.

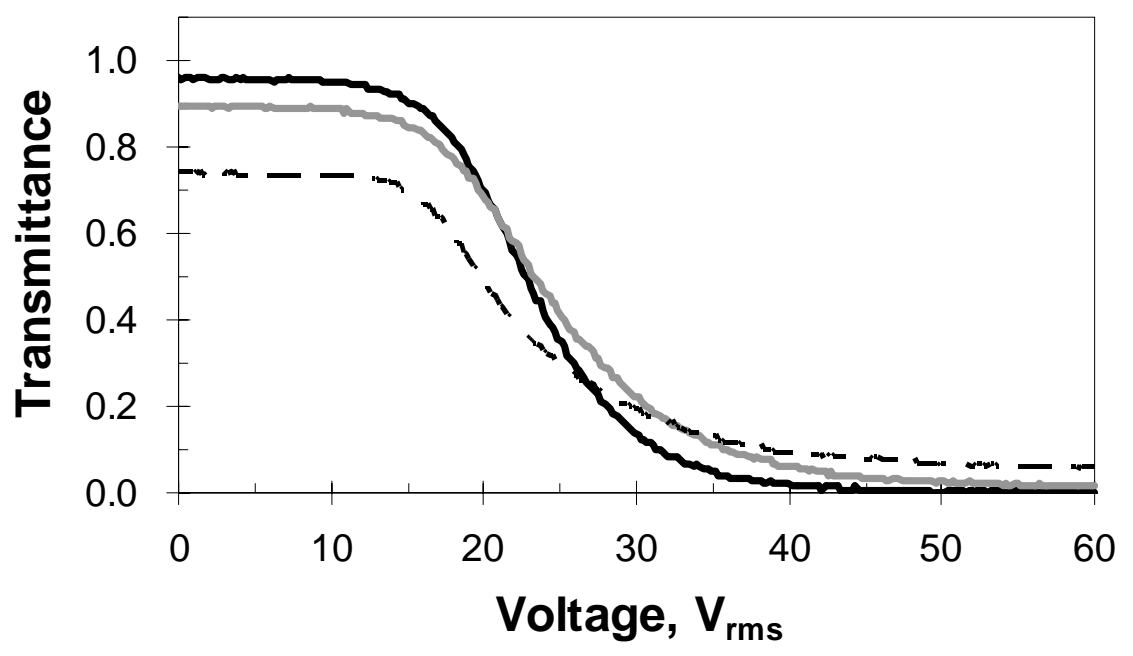

Figure 6.8: Voltage-dependent normalized transmittance of the DFLC gels with monomer concentration of $5 \mathrm{wt} \%$ at $15 \mathrm{~V}_{\text {rms }}$ (solid lines), $8 \mathrm{~V}_{\text {rms. }}$ (gray lines), and $4 \mathrm{~V}_{\text {rms }}$ (dashed lines) bias voltage during UV illumination.

The bias voltage during the curing process also affects the performance of the DFLC gels. Figure 6.8 plots the voltage-dependent transmittance of the $8-\mu \mathrm{m}$ DFLC gels at 4,8 , and $15 \mathrm{~V}_{\text {rms }}$ bias voltage. When the bias voltage is sufficiently higher than the threshold of the pure LC, most of the bulk LC molecules are reoriented perpendicular to the substrates. The resultant polymer networks are in a more ordered structure. If the bias voltage is too low, the cured LC molecules have a tilt angle with respect to the substrates. Its bright and dark states are not as good as those with high bias voltage, as depicted in Figure 6.8. 
To fabricate a polarization-independent DFLC gel, we inject the LC/monomer mixture into a cell without polyimide alignment layer. Figure 6.9 plots the voltage dependent transmittance of such a DFLC gel. The monomer concentration is $5 \mathrm{wt} \%$ and cell gap $d=8 \mu \mathrm{m}$. At $V=0$, the DFLC gel is in the uniform homeotropic state, similar to that shown in Figure 6.7(a).

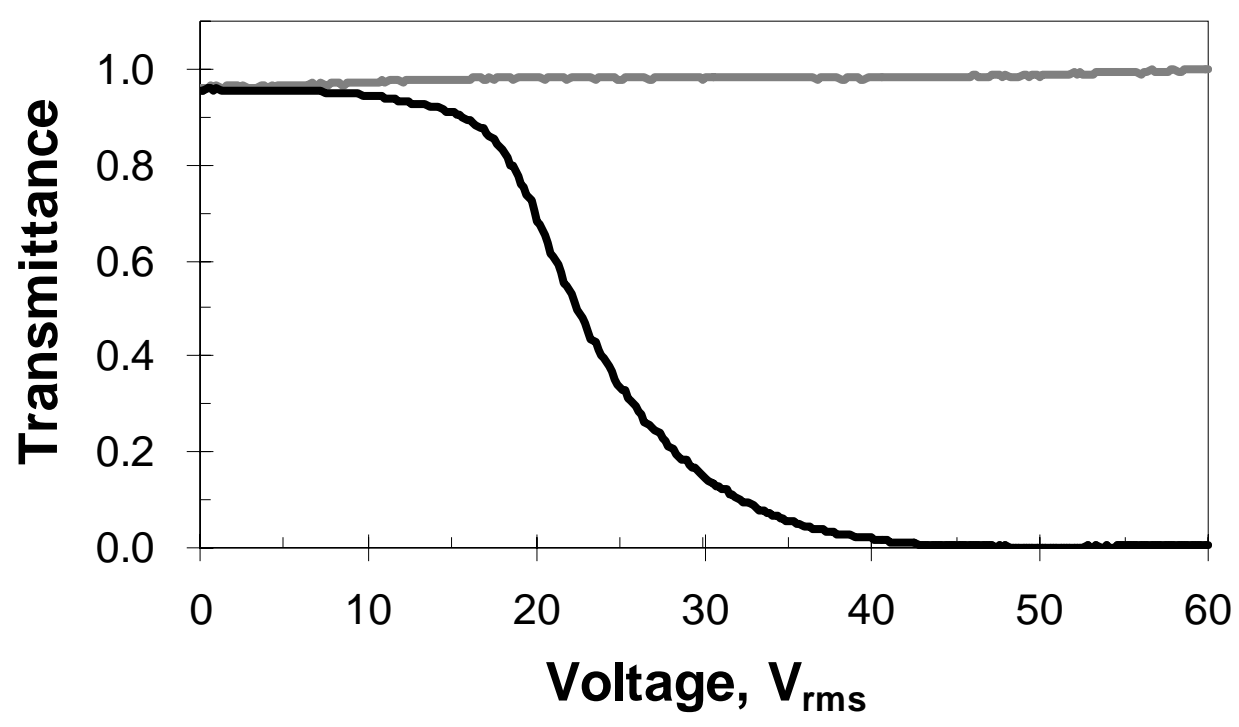

Figure 6.9: Voltage-dependent normalized transmittance of the no-alignment DFLC gels with monomer concentration of $5 \mathrm{wt} \%$ in an $8-\mu \mathrm{m}$ cell.

The incident light propagates through the cell without scattering. When we apply an electric field with $f=50 \mathrm{kHz}$, the LC molecules are tilted away from the field direction. The LC/polymer composite is switched to a multi-domain structure. The gel becomes optically nonuniform and therefore scatters the incident light. Since there is no preferred tilt direction, the LC directors in the polymer network domains are randomly distributed in the $x-y$ plane, as shown in Figure 6.10. The gel is polarization-independent 
and can be used as a broadband light shutter. For the $c=5$ wt $\%$ gel with $d=8 \mu \mathrm{m}$, the contrast ratio was measured to be $\sim 160: 1$ for the unpolarized He-Ne laser beam. A better dark state can be achieved at a higher voltage.

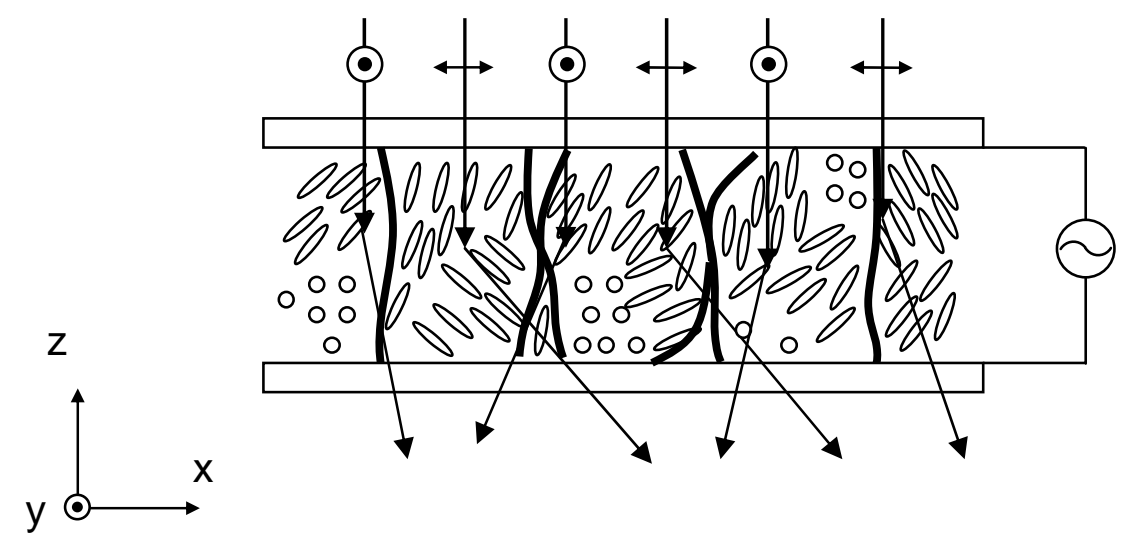

Figure 6.10: Schematic representation of the operating principle of no-alignment DFLC gels in a voltage-on state. The frequency of applied voltage is $50 \mathrm{kHz}$.

The response time of such an isotropic DFLC gel was measured. If we switch the applied voltage $(f=50 \mathrm{kHz})$ from 0 to $50 \mathrm{~V}_{\mathrm{rms}}$, the rise time is $0.8 \mathrm{~ms}$ and decay time is $10 \mathrm{~ms}$ for the $c=5 \mathrm{wt} \%$ sample. The observed response time of the isotropic gel is about 2X faster than the corresponding homogeneous DFLC gel. In the homogeneous cell, the buffed polyimide layers tend to align LC molecules parallel to the rubbing direction, but the polymer networks draw the LC directors perpendicular to the substrate. These two competing torques lead to a slower relaxation time. When we switch the isotropic gel at $50 \mathrm{~V}_{\text {rms }}$ between $f=1 \mathrm{kHz}$ and $50 \mathrm{kHz}$, the rise time and decay time is each $0.8 \mathrm{~ms}$. Without polyimide layer, the fabrication process of the isotropic gel is much simpler. 


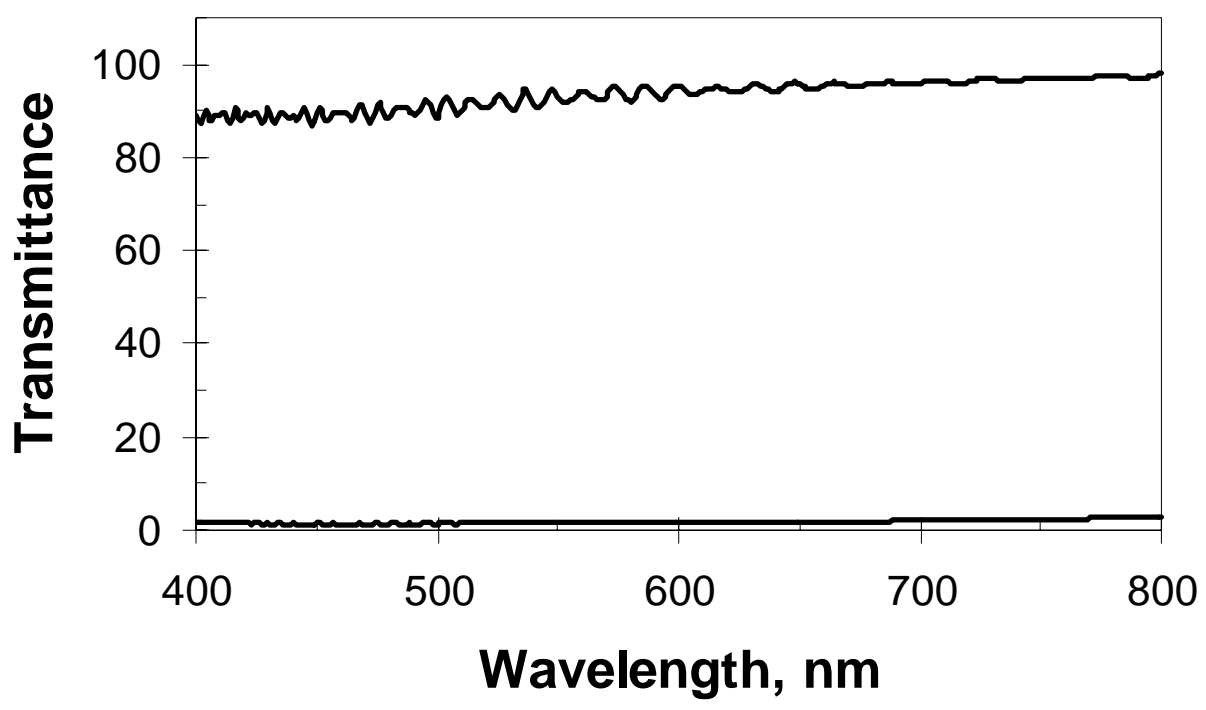

Figure 6.11: The spectral bandwidth of an 8- $\mu \mathrm{m}$ no-alignment DFLC gel. Upper trace: $V$ $=50 \mathrm{~V}_{\mathrm{rms}}$ at $f=1 \mathrm{kHz}$; lower trace: $V=50 \mathrm{~V}_{\mathrm{rms}}$ at $f=50 \mathrm{kHz}$.

Figure 6.11 shows the measured transmission spectra of the $8 \mu \mathrm{m}$ isotropic DFLC gel at voltage-on and -off states. The bright state was measured at $V=50 \mathrm{~V}_{\text {rms }}$ and $f=1$ $\mathrm{kHz}$ while the dark state was measured at the same voltage except for $f=50 \mathrm{kHz}$. Recall that the crossover frequency is $f_{c}=5 \mathrm{kHz}$. The spectral bandwidth covers the whole visible spectral region.

\subsubsection{Conclusion}

Two types of gels using dual-frequency liquid crystal (DFLC) are demonstrated. The one using a homogeneous cell shows anisotropic light scattering behavior while the other prepared using a cell without polyimide alignment layers exhibits isotropic light scattering properties. Both LC gels are highly transparent in the voltage-off state. Light 
scattering occurs when a high frequency voltage is applied. The isotropic gel exhibits a high contrast ratio and submillisecond response time. Potential applications of these gels for switchable polarizer, telecom optical switch, and reflective displays are emphasized.

In conclusion, we have demonstrated an anisotropic gel and an isotropic gel using a dual-frequency LC. The light scattering behavior for the homogeneous LC gel is dependent on the incident light polarization. However, the gel without alignment layer is polarization independent. These devices exhibit a high contrast ratio and fast response time. These LC gels can be used as a switchable polarizer and light shutter. 


\section{CHAPTER 7: FAST-RESPONSE AND SCATTERING-FREE POLYMER NETWORK LIQUID CRYSTALS FOR INFRARED LIGHT MODULATORS}

\subsection{Introduction: Fast-Switching Liquid Crystal Devices}

Liquid crystal/polymer composites could appear in network or droplet form depending on what polymer and concentration is employed. In the low concentration regime $(<8 \mathrm{wt} \%),{ }^{23-26}$ the monomer molecules link together to form a chain-like polymer network during polymerization process. The polymer networks are like fibril-bundle with nano-sized diameter. ${ }^{98}$. The direction of the polymer bundle is controlled by the surface alignment layer or external field. Liquid crystal molecules are filled in the space between the polymer fibrils. The polymer networks will prevent the change of the LC directors. Therefore, the polymer field improves the response time; however, the associated light scattering is quite strong in the visible region. The polymer network technique has been introduced to decrease the rise time of the IPS (In-plane-switching) mode from $\sim 90 \mathrm{~ms}$ in a pure liquid crystal to $\sim 10 \mathrm{~ms}$ in $2 \%$ polymer network liquid crystal composites. ${ }^{23}$. However, the light scattering reduces the transmission and degrades the image quality.

As the polymer concentration increases to $\sim 30-40 \%$, the polymer-dispersed liquid crystal (PDLC) is formed. ${ }^{17,20}$. No surface alignment in the PDLC cell is necessary. These micron-sized droplets scatter visible light and this scattering behavior is independent of polarization. In the high polymer concentration regime $(60-70 \%)$, the nanoscale 
holographic PDLC (HPDLC) droplets are formed. ${ }^{11,99}$. Since the droplet size is smaller than the visible wavelength, HPDLC cell does not scatter light and its response time is fast ( $200 \mu \mathrm{s})$. However, to reorient these LC droplets would require a relatively high electric field $\left(\sim 15-20 \mathrm{~V}_{\mathrm{rms}} / \mu \mathrm{m}\right)$.

Recently, a stressed LC cell comprising of 14 wt \% Nolan-65 polymer was demonstrated to exhibit a fast response time. ${ }^{100}$. The oriented liquid crystal domains are formed by mechanical shearing the polymer-dispersed liquid crystal. ${ }^{101,102}$. In this approach, no surface alignment is required. However, a delicate shearing process is needed in order to suppress light scattering. The operating voltage for a $20 \mu \mathrm{m}$ E7 cell is $\sim 135 \mathrm{~V}_{\text {rms. }}$

In this work, we report a fast-response homogeneously-aligned polymer network liquid crystal (PNLC) cell comprising of $\sim 10 \%$ polymers. A unique feature of this PNLC is that light scattering at $\lambda=1.55 \mu \mathrm{m}$ is suppressed completely. The measured response time is less than $2 \mathrm{~ms}$ for a $2 \pi$ phase change at $\lambda=1.55 \mu \mathrm{m}$ and $T \sim 21^{\circ} \mathrm{C}$. The required electric field strength for the PNLC cells is $\sim 7-8 \mathrm{~V}_{\mathrm{rms}} / \mu \mathrm{m}$. To reduce operating voltage, a thicker cell or higher birefringence LC can be considered.

\section{$\underline{7.2 \text { Experimental Methods }}$}

To fabricate the PNLC cells, we first mixed a few percent of photocurable monomer to a nematic LC host. The LC/monomer composite was sandwiched between two glass substrates. The inner surfaces of the indium-tin-oxide (ITO) glass substrates were over-coated with a thin polyimide layer and buffed in anti-parallel directions for achieving homogeneous alignment. The filled cell was then exposed to UV light for 
curing the polymer networks. To eliminate light scattering, we need to control the domain sizes to be smaller than the wavelength. A weak UV intensity would result in coarser polymer networks which, in turn, lead to larger domain sizes. Thus, we exposed the LC/monomer mixture using a high UV intensity $\left(I \sim 45 \mathrm{~mW} / \mathrm{cm}^{2}\right)$. The exposure time is $30 \mathrm{~min}$. The resultant polymer networks align, on the average, parallel to the substrate surfaces due to the strong surface anchoring energy. Thus, the PNLC cell is highly transparent in the voltage-off state. In a voltage-on state, the polymer networks exert a torque to resist the $\mathrm{LC}$ molecules from being reoriented by the electric field. As a result, the threshold voltage is increased significantly. Once the electric field is removed, the strong polymer field assists LC to relax back quickly.

For laser beam steering at $\lambda=1.55 \mu \mathrm{m}$, both reflective and transmissive phase modulators can be used. ${ }^{22}$. To generate diffractive phase gratings, a $2 \pi$ phase change between adjacent grating elements is required. The reflective and transmissive PNLC cells have been prepared and investigated.

Figure 7.1 depicts the experimental setup for studying the electro-optic effects of the reflective and transmissive PNLC cells. The incoming diode laser beam $(\lambda=1.55 \mu \mathrm{m})$ is reflected or transmitted by the polarizing beam splitter (PBS) to the PNLC cell. The rubbing direction of the $\mathrm{LC}$ cell is oriented to be $45^{\circ}$ to the polarization axis of the incident beam. For the reflective mode, ideally the reflector should be imbedded in the inner side of the rear substrate in order to eliminate multiple surface reflections. For feasibility studies, we placed a dielectric mirror behind the LC cell to serve as a reflector. For the transmissive mode, the analyzer is crossed to the polarizer. The voltage- 
dependent reflectance or transmittance of the cells is recorded by the LabVIEW system and data are analyzed by a computer.

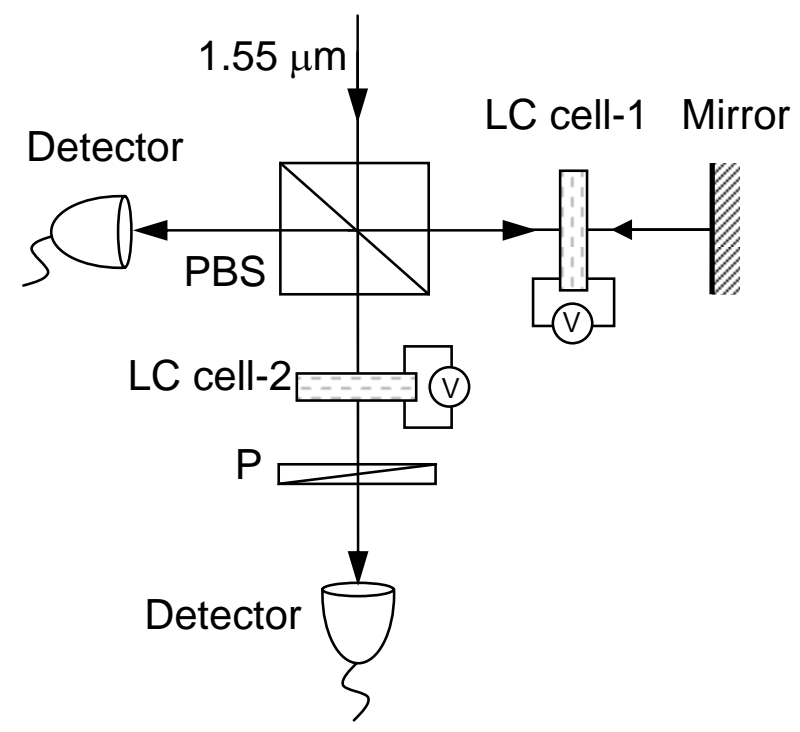

Figure 7.1: Experimental setup for measuring the electro-optic properties of the reflective and transmissive LC and PNLC cells. The rubbing direction of the cell is at $45^{\circ}$ to the polarization axis of the incident light. The LC cell-1 and detector 1 are for reflective mode and LC cell-2 and detector 2 are for transmissive mode. PBS = polarizing beam splitter. $\mathrm{P}=$ polarizer. $\lambda=1.55 \mu \mathrm{m}$.

Two types of diacrylate monomers: bisphenol-A-dimethacrylate (M1) and RM-82 (Merck) were used. They have a rod-like structure. The melting point of these two monomers is 74.6 and $82.4{ }^{\circ} \mathrm{C}$, respectively. Due to the high heat fusion enthalpy, each monomer's solubility is limited to $7-8 \mathrm{wt} \%$. Figure 7.2 shows the voltage-dependant reflectance of PNLC cells with different monomer concentrations. When monomer concentration is $7 \mathrm{wt} \%$, the cured polymer network domain size is still too large which results in $\sim 10 \%$ light scattering loss at $\lambda=1.55 \mu \mathrm{m}$. In order to eliminate light scattering, we need to reduce the domain size further by increasing the monomer concentration. 
Therefore, we mixed M1 with RM-82 to lower the melting point. For the LC mixture (Merck E44) we studied, the best monomer concentration is $6 \mathrm{wt} \% \mathrm{M} 1$ and $4 \mathrm{wt} \% \mathrm{RM}$ 82, shown as the black solid line in Figure 7.2. Unless otherwise mentioned throughout this paper, our LC/polymer contains 90\% LC, 6\% M1 and 4\% RM- 82 .

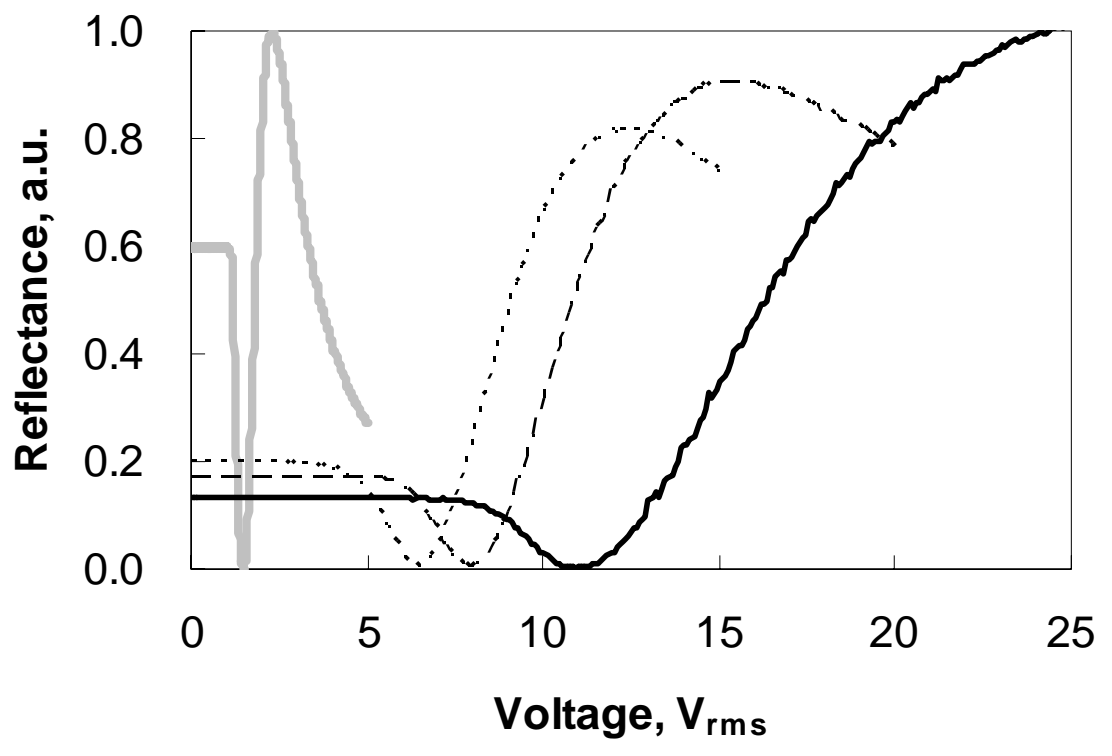

Figure 7.2: Voltage-dependent reflectance of E44 (gray lines) and PNLC (dark lines) cells. From left to right, $c=5,7$, and $10 \mathrm{wt} \%$. The cell gap for the reflective cells is $5 \mu \mathrm{m}$. $\lambda=1.55 \mu \mathrm{m}$ and $T=21^{\circ} \mathrm{C}$.

\section{$\underline{7.3 \text { Experimental Results and Discussions }}$}

Figures 7.3(a) and (b) plot the voltage-dependent reflectance and transmittance of the corresponding $7.7 \mu \mathrm{m}$ and $12.5 \mu \mathrm{m}$ E44 PNLC cells. The total phase retardation of the reflective and transmissive PNLC cells is measured to be $\delta=3.84 \pi$ and $3.0 \pi$, respectively. However, the last $\pi$ phase change would require a very large voltage swing. For the interest of keeping operating voltage low, we operated the LC modulator from $2 \pi$ 
to 0. Also included in Figures 7.3(a) and (b) (gray lines) for comparisons are the 7.7- $\mu \mathrm{m}$ and $12.5-\mu \mathrm{m}$ E44 cells without polymer. The threshold voltage of the E44 cells is $\sim 0.9$ $\mathrm{V}_{\text {rms. }}$. From the measured total phase retardation, the birefringence of E44 at $\lambda=1.55 \mu \mathrm{m}$ is $\Delta n \sim 0.21$ at $T \sim 21{ }^{\circ} \mathrm{C}$. On the other hand, the $\Delta n$ of the PNLC cell was measured to be $\Delta n \sim 0.19$. The lower $\Delta n$ originates from the imbedded $10 \%$ polymers. This result indicates that the polymer networks do not disturb LC alignment noticeably. The threshold voltage of the 7.7- $\mu \mathrm{m}$ and $12.5-\mu \mathrm{m}$ PNLC cells is increased to 20 and $\sim 24 \mathrm{~V}_{\mathrm{rms}}$, respectively. The increased threshold voltage implies that the LC molecules are tightly anchored by the polymer networks. In Figures 7.3(a) and (b), the peak transmittance of the PNLC cells is similar to that of the pure E44 cells. This indicates that the light scattering is eliminated. We also measured the wavelength-dependent transmittance of the $12.5 \mu \mathrm{m}$ cell. Scattering is strong in the visible region but becomes negligible at $\lambda>1 \mu \mathrm{m}$.

Figures 7.4(a) and (b) show the measured decay time (solid lines) of the reflective and transmissive PNLC cells. The open circles are fittings with exponential phase decay: ${ }^{103}$

$$
\delta(t)=2 \pi \exp (-t / \tau)
$$

Good agreement is found. The time constant (at $1 / \mathrm{e}$ ) for the $7.7-\mu \mathrm{m}$ reflective cell and 12.5- $\mu \mathrm{m}$ transmissive cell is $\tau=296 \mu \mathrm{s}$ and $755 \mu \mathrm{s}$, respectively. If we count the phase decay time from $100 \rightarrow 10 \%$, they are $\sim 0.7 \mathrm{~ms}$ and $1.8 \mathrm{~ms}$, respectively. As compared to the pure E44 cell, the response time is improved by $\sim 300 \mathrm{X}$. The rise time $(10 \rightarrow 90 \%$ phase change) was measured to be $\sim 0.5 \mathrm{~ms}$ for the transmissive PNLC cell operating from 0 to $90 \mathrm{~V}_{\mathrm{rms}}$. 


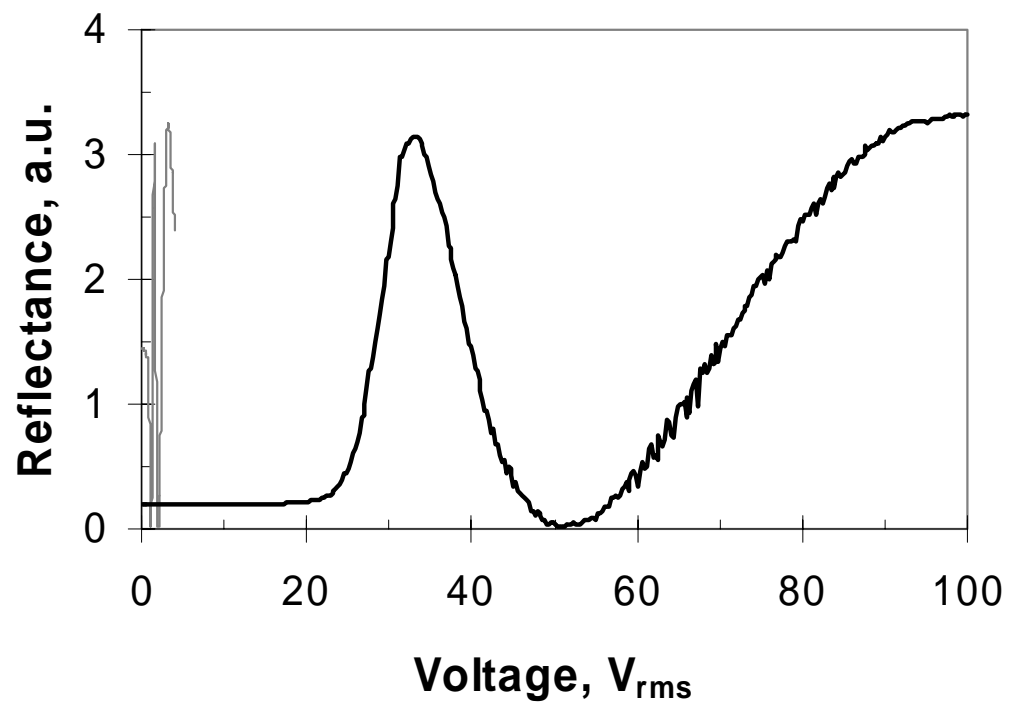

(a)

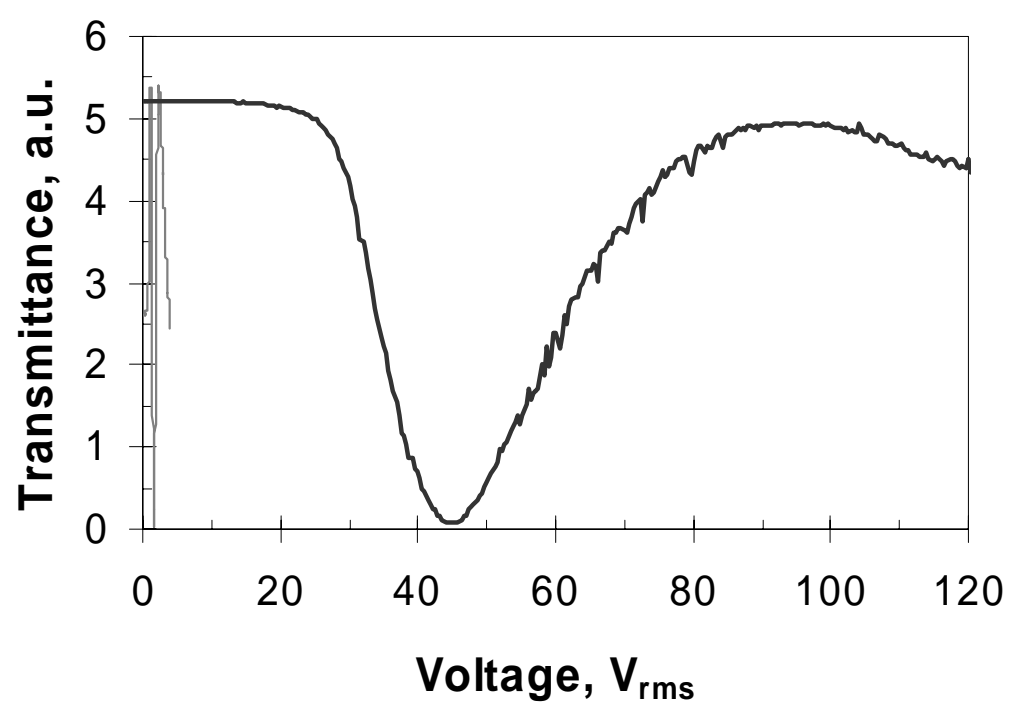

(b)

Figure 7.3: Voltage-dependent reflectance (a) and transmittance (b) of E44 (gray lines) and PNLC (dark lines) cells. The cell gap for the reflective and transmissive cells is 7.7 and $12.5 \mu \mathrm{m}$, respectively. $\lambda=1.55 \mu \mathrm{m}$ and $T=21^{\circ} \mathrm{C}$. 


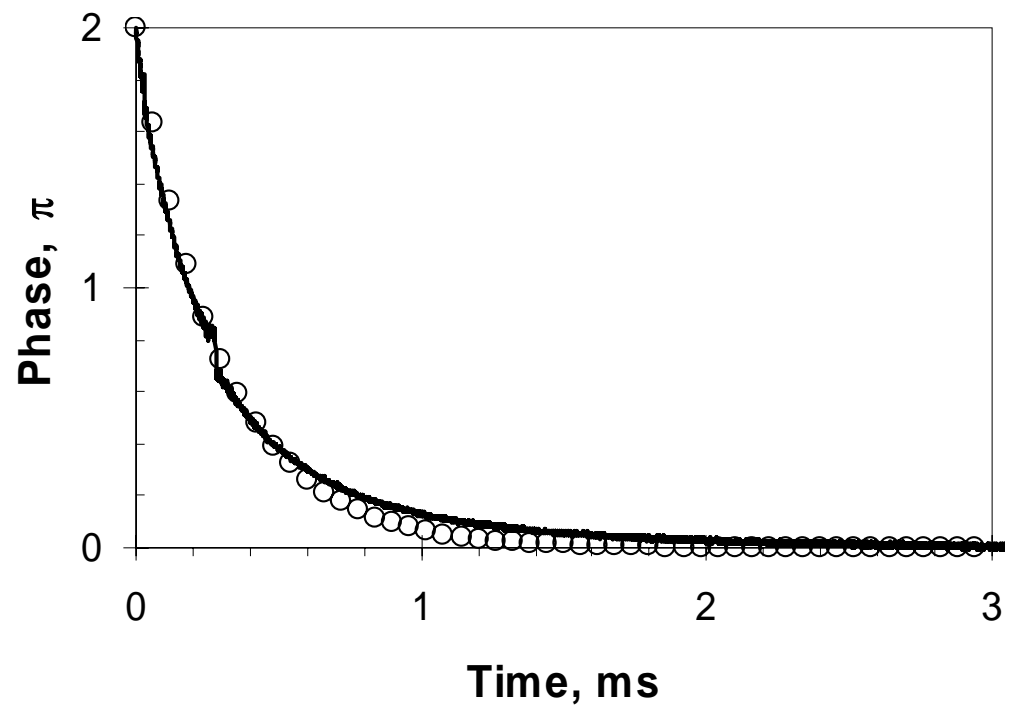

(a)

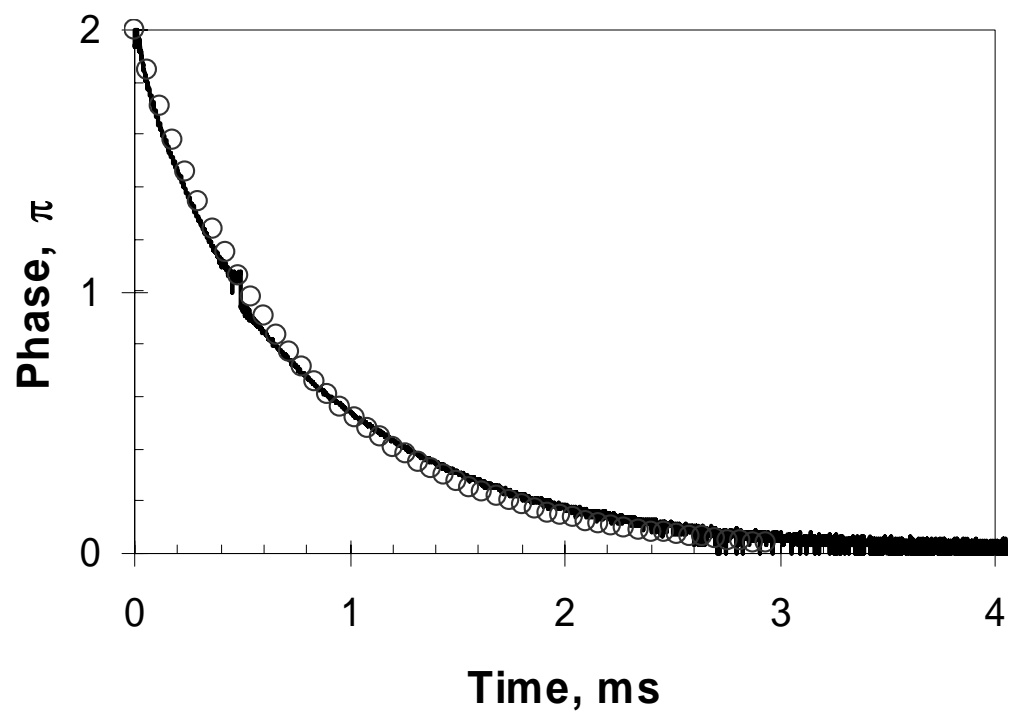

(b)

Figure 7.4: Measured phase decay time of the reflective (a) and transmissive (b) PNLC cells. Solid lines are experimental data and open circles are fitting results using $\delta(\mathrm{t})=2 \pi$ $\exp (2 t / \tau)$. In (a), $\tau=296 \mu$ s and in (b) $\tau=755 \mu$ s. $T=21{ }^{\circ} \mathrm{C}$ and $\lambda=1.55 \mu \mathrm{m}$. 


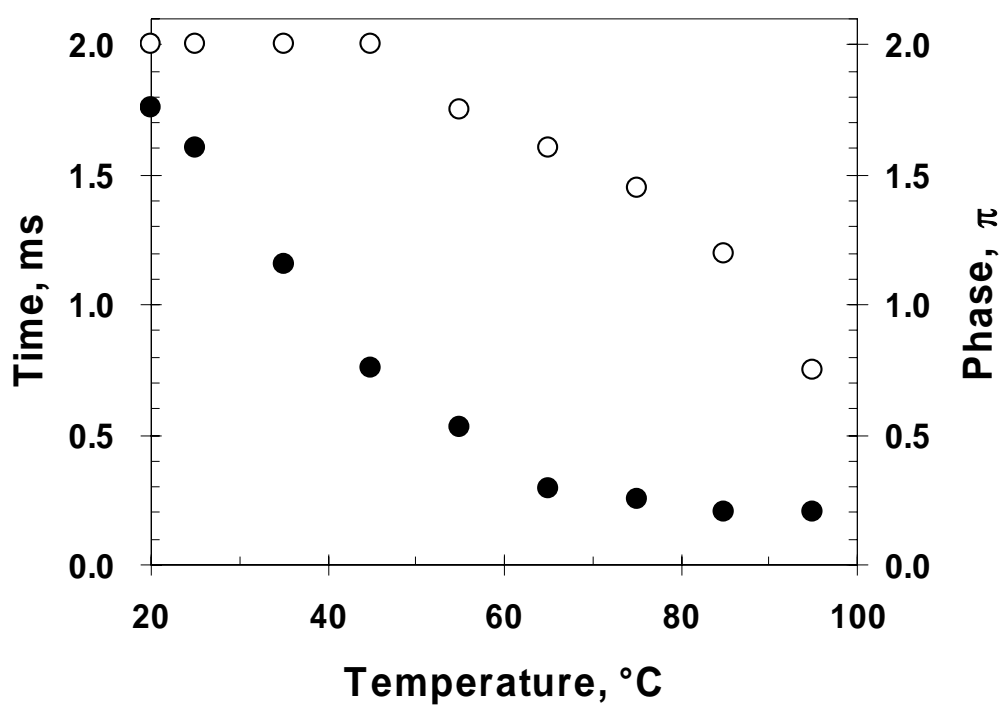

(a)

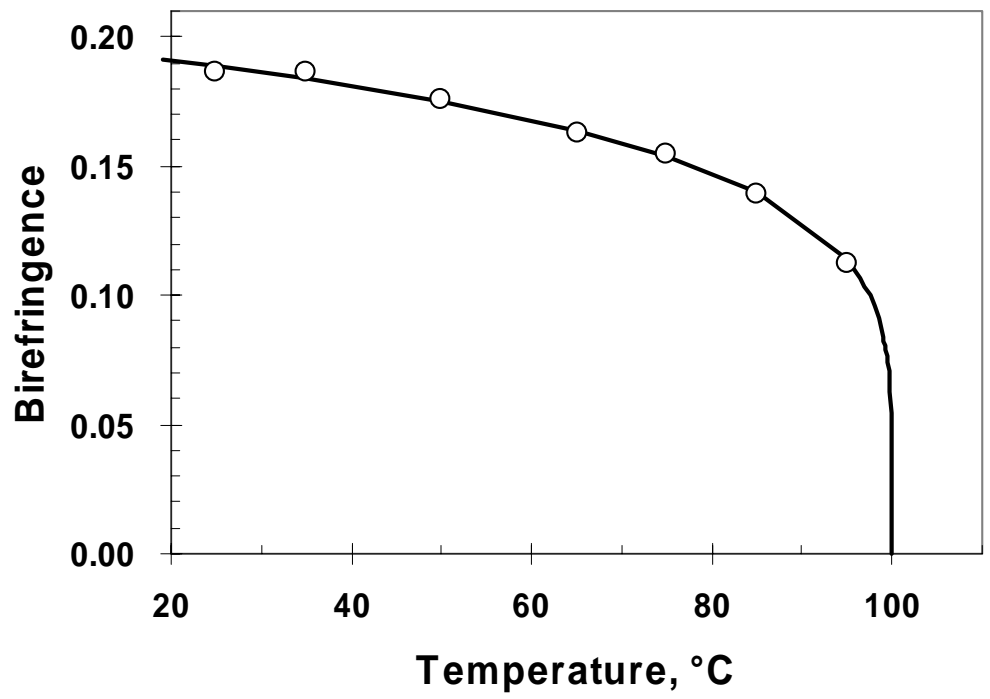

(b)

Figure 7.5: Temperature-dependent LC response time (a) and birefringence (b) of the E44 transmissive PNLC cell. $D=12.5 \mu \mathrm{m}$. Closed circles are the measured response time and open circles are the corresponding phase change. 
Figure 7.5(a) plots the temperature dependent decay time (filled circles) and the corresponding phase (open circles) of the $12.5 \mu \mathrm{m}$ transmissive PNLC cell. As the temperature increases, the operating voltage, birefringence and response time all decrease. At $T=65{ }^{\circ} \mathrm{C}$, the decay time for the transmissive PNLC cell is reduced to $\sim 300 \mu \mathrm{s}$, although the phase change studied is reduced to $1.6 \pi$. Figure 10.5(b) shows the temperature dependent birefringence of the transmissive E44 PNLC cell at $\lambda=1.55 \mu \mathrm{m}$. The dots represent the experimental data and solid lines are fittings with Haller's equation:

$$
\Delta n=\Delta n_{o} \cdot\left(1-T / T_{c}\right)^{\beta}
$$

where $\Delta n_{o}$ is the birefringence at $T=0$ and $\beta$ is a material parameter. ${ }^{104}$. From the fittings, we find $\Delta n_{o}=0.255$ and $\beta=0.187$.

\section{$\underline{7.4}$ Numerical Calculations and Simulations}

To explain the mechanism of fast-switching speed of PNLC cell, we calculate voltage-induced LC director numerically and compare the results with those of the pure E44 cell. We start by calculating the voltage-dependent refractive index change for a symmetric, uniform liquid crystal layer and use that to predict the voltage-dependent transmittance. The LC directors are reoriented to align with the electric field $(\boldsymbol{E})$ in order to minimize the free energy. The LC director distribution is calculated by Eqs. (4.4) and (4.5) in the static electric field. Using the tilt angle profile $\theta(z)$ and the refractive index for a given tilt angle from Eq. (4.12), the average refractive index, $\widetilde{n}$, of the liquid crystal can be obtained at different applied voltages. 
Table 7.1 Physical parameters of E44 for simulation

\begin{tabular}{|c|c|}
\hline E44 parameters & \\
\hline Splay elastic constants, $k_{11}$ & $15.3 \mathrm{pN}$ \\
Bend elastic constants, $k_{33}$ & $24.2 \mathrm{pN}$ \\
Parallel dielectric constant, $\varepsilon_{/ /}$ & 20 \\
Perpendicular dielectric constant, $\varepsilon_{\perp}$ & 4.5 \\
Rotational viscosity constant, $\gamma_{1}$ & 0.448 Pas \\
Birefringence, $\Delta n$ & 0.21 \\
\hline
\end{tabular}

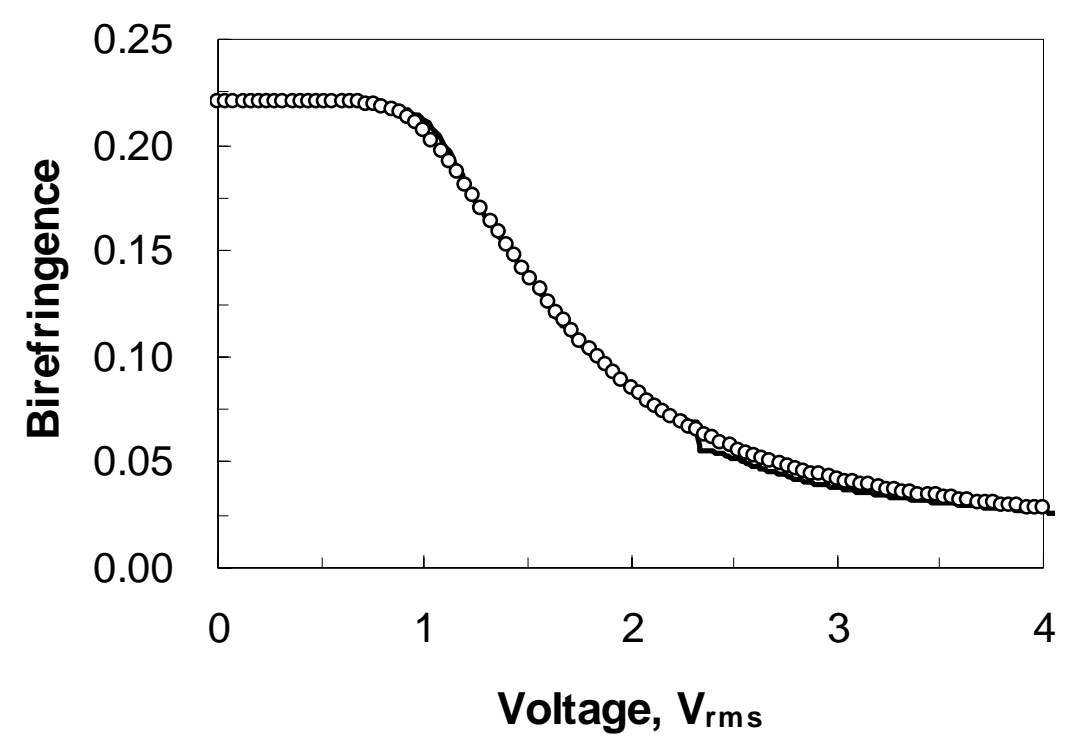

Figure 7.6: Voltage-dependent average refractive index of dual-frequency liquid crystal used in the experiment. Solid line and open circles represent the experimental and simulation results, respectively. $\lambda=1.55 \mu \mathrm{m}$. 
For the E44 cell, the voltage-dependent average effective refractive index was calculated and plotted in Figure 7.6; the following measured parameters are used: $\Delta \varepsilon=15.5, k_{11}=15.3 \mathrm{pN}$, and $\gamma_{1}=0.448$ Pas. The ratio of $\gamma_{1} / k_{11}$ is $29.33 \mathrm{~ms} / \mu \mathrm{m}^{2}$. We assumed the pretilt angle is $2.5^{\circ}$. From fitting, we find $k_{33}=24.2 \mathrm{pN}$. The voltagedependent transmittance of the E44 cell is shown in Figure 7.7(a).

Table 7.2 Physical parameters of PNLC sample for simulation

\begin{tabular}{|c|c|}
\hline PNLC sample parameters & \\
\hline Effective splay elastic constants, $k_{11}$ & $4,800 \mathrm{pN}$ \\
Effective bend elastic constants, $k_{33}$. & $50,000 \mathrm{pN}$ \\
Parallel dielectric constant, $\varepsilon_{/ \cdot}$ & 20 \\
Perpendicular dielectric constant, $\varepsilon_{\perp}$ & 4.5 \\
Rotational viscosity constant, $\gamma_{1}$ & 0.448 Pas \\
Birefringence, $\Delta n$ & 0.19 \\
\hline
\end{tabular}




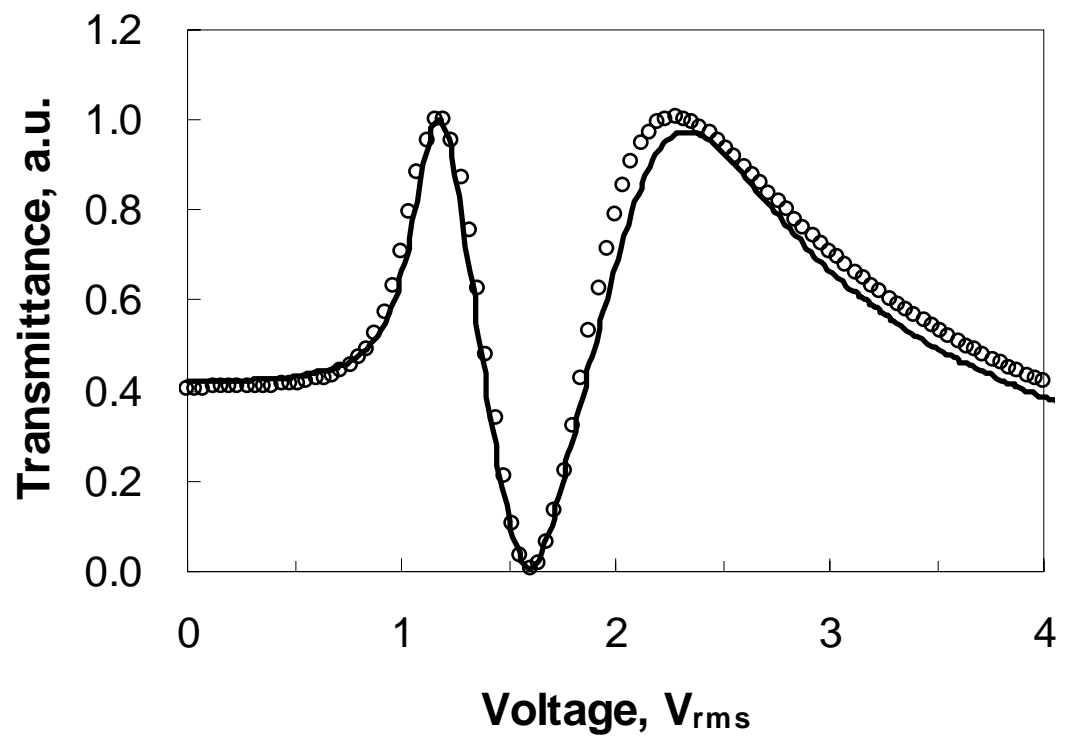

(a)

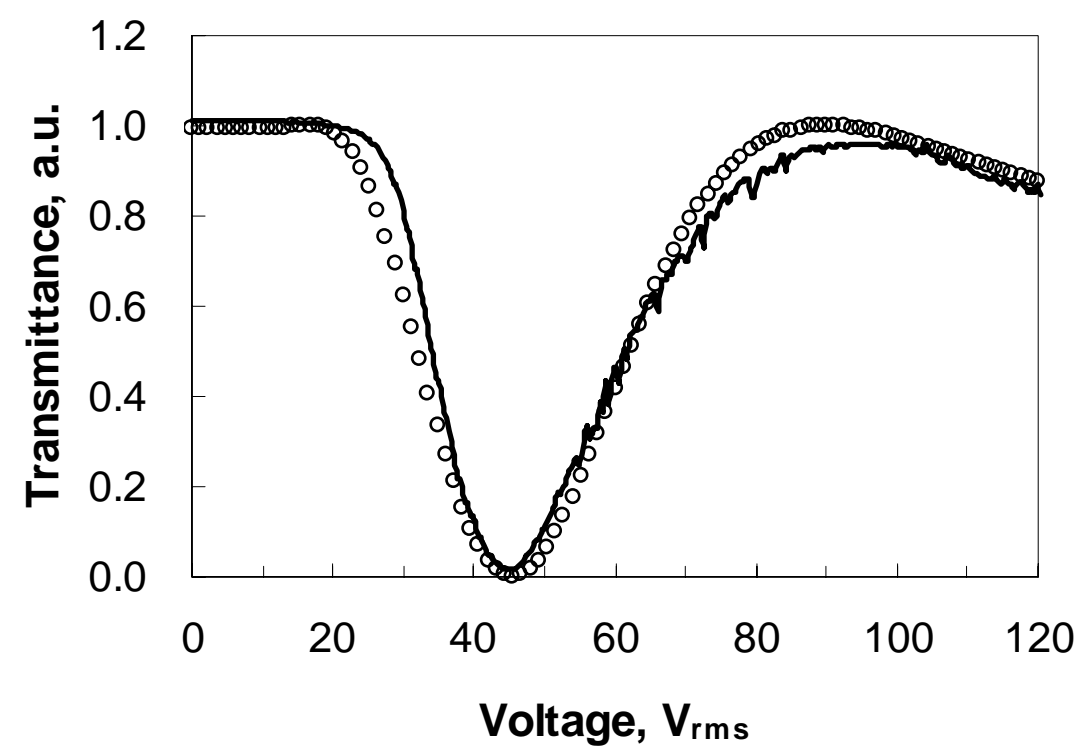

(b)

Figure 7.7: The simulation results of E44 (a) and PNLC (b) cells. Solid lines are experimental data and open circles are fitting results. $T=21^{\circ} \mathrm{C}$ and $\lambda=1.55 \mu \mathrm{m}$. 
For the PNLC cell, we assume the $\Delta \varepsilon$ and $\gamma_{1}$ of E44 do not change because the pure LC molecules are completely separated from the polymer. In the PNLC cell, the measured $\gamma_{1} / k_{11}$ drops to $0.094 \mathrm{~ms} / \mu \mathrm{m}^{2}$, indicating a much faster decay time. The expected effective $k_{11}$ of PNLC cell would be 4773 pN. From the fittings shown in Figure 7.7(b), we find $k_{11}=4800 \mathrm{pN}$ and $k_{33}=50000 \mathrm{pN}$. The simulation results imply that the strong anchoring force of polymer network in bulk LC area dramatically increases the effective $k$ value. The LC molecules in the polymer network domains become stiffer and more difficult to be reoriented by the electric field. A small portion of the bulk LC molecules near the boundaries of the network domain may not rotate at all, which results a slower phase change in the high voltage regime.

\subsection{Methods of Reducing Operating Voltage}

To reduce the operating voltage, we cured the PNLC cell under a bias voltage $V_{b}$. The $8-\mu \mathrm{m}$ PNLC cells were prepared at $V_{b}=0,0.9,1.0$, and $1.1 \mathrm{~V}_{\mathrm{rms}}$, as shown in Figure 7.8. The high threshold voltage is reduced when $V_{b}=0.9 \mathrm{~V}_{\mathrm{rms}}$ and eliminated when $V>$ $1.0 \mathrm{~V}_{\mathrm{rms}}$. The tradeoff is that the response time is slightly increased. The bias voltage needs to be optimized in order to maintain the maximum phase change and fast response time.

To reduce the operating voltage while retaining the required $2 \pi$ phase change, we could increase the LC birefringence, dielectric anisotropy $(\Delta \varepsilon)$, or cell gap. Among these three, the simplest method is to increase cell gap. In experiment, we tried a 16- $\mu \mathrm{m} \mathrm{E44}$ PNLC cell. Indeed, its operating voltage at $\delta=2 \pi$ is decreased to $\sim 75 \mathrm{~V}_{\text {rms }}$, however, its decay time is increased to $\sim 3 \mathrm{~ms}$. Our experimental data show that the response time of 
the PNLC cell is linearly proportional to the cell gap. Thus, for practical applications a proper balance between operating voltage and response time needs to be taken into consideration.

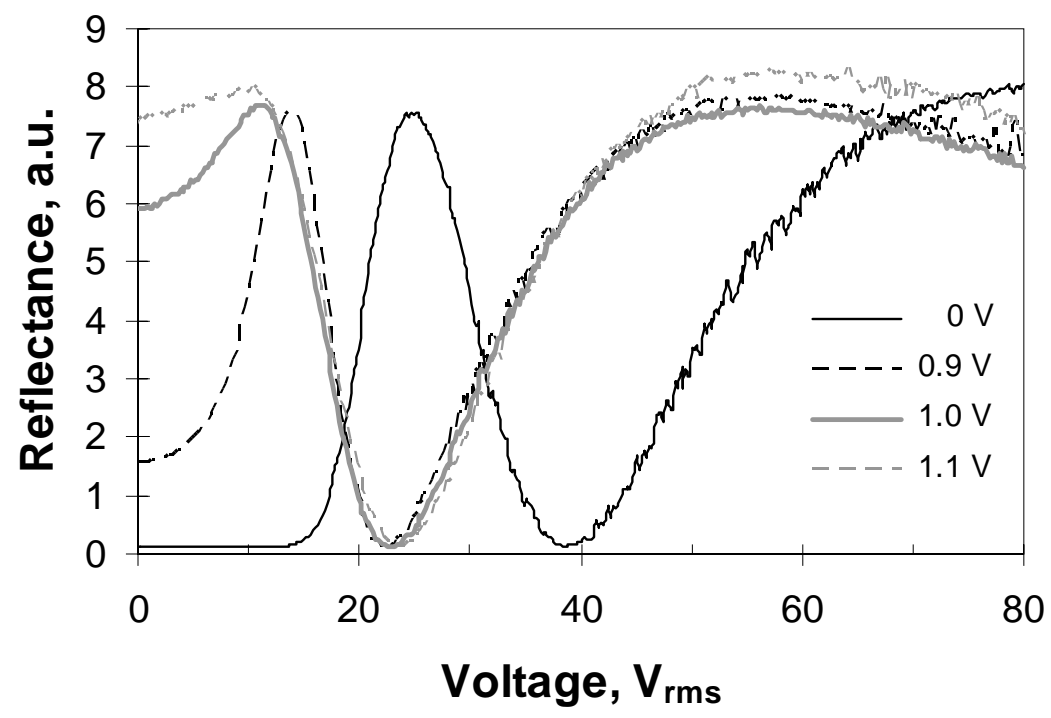

Figure 7.8: Voltage-dependent reflectance PNLC cells cured with different bias voltage. The cell gap for the reflective cells is $8 \mu \mathrm{m} . T=21^{\circ} \mathrm{C}$ and $\lambda=1.55 \mu \mathrm{m}$.

Figure 7.9 shows the simulation results of the PNLC cell as $\Delta n$ and $\Delta \varepsilon$ increase.

The $V_{2 \pi}$ could be reduced to $54.5 \mathrm{~V}_{\mathrm{rms}}$ if $\Delta \mathrm{n}$ is increased to 0.3, as shown in Figure 7.9(a). We could lower both $V_{\text {th }}$ and $V_{2 \pi}$ by using a higher $\Delta \varepsilon$ material. The $V_{2 \pi}$ is decreased gradually as $\Delta \varepsilon$ increases, as shown in Fig. 7.9(b). 


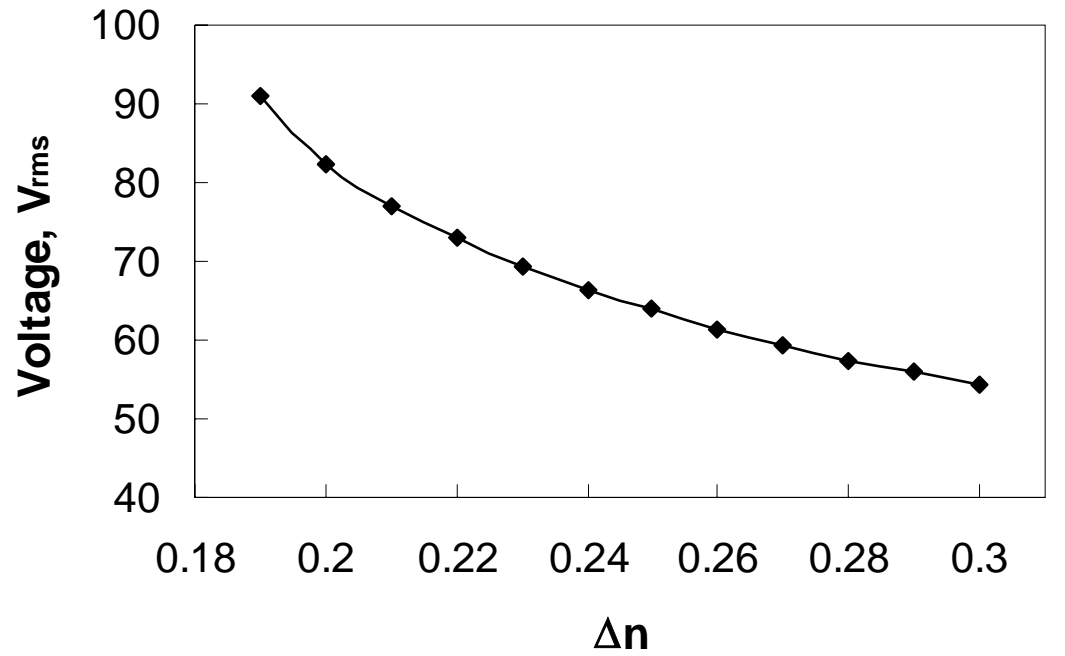

(a)

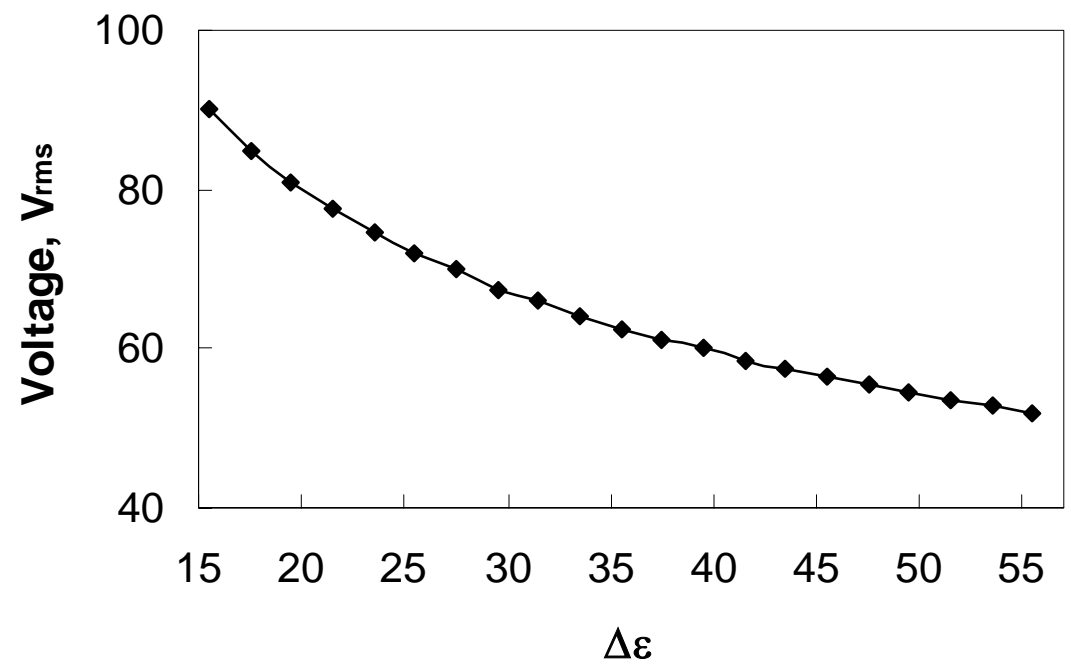

(b)

Figure 7.9: The simulation results of PNLC cell with different $\Delta n$ (a) and $\Delta \varepsilon$ (b). The fitting parameters: $k_{11}=4800 \mathrm{pN}$, and $k_{33}=50000 \mathrm{pN}$. $\lambda=1.55 \mu \mathrm{m}$. 
The developed PNLC can also be extended to the 3-5 and 8-12 $\mu \mathrm{m}$ spectral bands. In the long wavelength region, the LC birefringence decreases ${ }^{105}$. but the light scattering becomes less significant. To obtain $2 \pi$ phase change, we need a thicker LC layer. The increased cell gap would lead to a higher operating voltage. To reduce voltage, we could increase the domain size, but the response time would increase accordingly.

\section{$\underline{7.6 \text { Conclusion }}$}

A fast-response and scattering-free homogeneously-aligned polymer network liquid crystal (PNLC) light modulator is demonstrated at $\lambda=1.55 \mu \mathrm{m}$ wavelength. Both reflective and transmissive PNLC phase modulators are investigated. Light scattering in the near-infrared region is suppressed by optimizing the polymer concentration such that the network domain sizes are smaller than the wavelength. The strong polymer network anchoring assists LC to relax back quickly as the electric field is removed. As a result, the PNLC response time is $\sim 300 \mathrm{X}$ faster than that of the E44 LC mixture except that the threshold voltage is increased by $\sim 25 \mathrm{X}$.

The physical properties of PNLC cell can be simulated by a standard onedimensional finite element method. The simulation results agree well with experiment. The temperature dependent birefringence of the PNLC cell is the same as the host liquid crystal. To reduce operating voltage, special curing environment under a bias voltage is proposed. To develop higher performance PNLC cell, liquid crystal materials with a high $\Delta n$ and large $\Delta \varepsilon$ would be desirable. The PNLC cell also holds promise for mid and long infrared applications. 


\section{LIST OF REFERENCES}

1 J. Chen, P. J. Bos, H. Vithana, and D. L. Johnson, "An electrooptically controlled liquid-crystal diffraction grating," Applied Physics Letters 67 (18), 2588-2590 (1995).

2 M. Ferstl and A. M. Frisch, "Static and dynamic fresnel zone lenses for optical interconnections," Journal of Modern Optics 43 (7), 1451-1462 (1996).

3 G. D. Love, J. V. Major, and A. Purvis, "Liquid-crystal prisms for tip-tilt adaptive optics," Optics Letters 19 (15), 1170-1172 (1994).

4 A. F. Naumov, M. Y. Loktev, I. R. Guralnik, and G. Vdovin, "Liquid-crystal adaptive lenses with modal control," Optics Letters 23 (13), $992-994$ (1998).

5 H. Okada, P. Bos, and H. Onnagawa, "In-plane liquid crystal beam steering devices with a beam separation structure," Japanese Journal of Applied Physics 37 (5A), 2576-2580 (1998).

6 J. S. Patel and K. Rastani, "Electrically controlled polarization-independent liquid-crystal fresnel lens arrays," Optics Letters 16 (7), 532-534 (1991).

7 V. V. Presnyakov, K. E. Asatryan, T. V. Galstian, and A. Tork, "Polymerstabilized liquid crystal for tunable microlens applications," Optics Express 10 (17), 865-870 (2002).

8 D. P. Resler, D. S. Hobbs, R. C. Sharp, L. J. Friedman, and T. A. Dorschner, "High-efficiency liquid-crystal optical phased-array beam steering," Optics Letters 21 (9), 689-691 (1996). 
9 N. A. Riza and M. C. Dejule, "3-terminal adaptive nematic liquid-crystal lens device," Optics Letters 19 (14), 1013-1015 (1994).

10 S. Sato, "Liquid-crystal lens-cells with variable focal length," Japanese Journal of Applied Physics 18 (9), 1679-1684 (1979).

11 R. L. Sutherland, V. P. Tondiglia, L. V. Natarajan, T. J. Bunning, and W. W. Adams, "Electrically switchable volume gratings in polymer-dispersed liquidcrystals," Applied Physics Letters 64 (9), 1074-1076 (1994).

12 X. Wang, D. Wilson, R. Muller, P. Maker, and D. Psaltis, "Liquid-crystal blazedgrating beam deflector," Applied Optics 39 (35), 6545-6555 (2000).

13 G. W. Gray and J. Goodby, Smectic liquid crystals: Textures and structures. (Leonard Hill, 1984).

14 P. G. d. Gennes and J. Prost, The physics of liquid crystals, 2nd ed. (Clarendon Press; Oxford University Press, Oxford, New York, 1993), pp.xvi, 597.

15 I. C. Khoo, Liquid crystals: Physical properties and nonlinear optical phenomena. (John Willy \& Sons, INC., New York, Chichester, Brisban, Toronto, Singapore, 1995).

16 F. C. Frank, "Liquid crystals: Theory of liquid crystals," Discuss. Faraday Soc. 25, 19 (1958).

17 J. L. Fergason, "Polymer encapsulated nematic liquid crystals for display and light control applications," SID Symposium Digest 16, 68-70 (1985).

18 C. Kittel and H. Kroemer, Thermal physics, 2d ed. (W., San Francisco, 1980), pp.xvii, 473. 
19 J. L. West, "Phase-separation of liquid-crystals in polymers," Molecular Crystals and Liquid Crystals 157, 427-441 (1988).

20 J. W. Doane, N. A. Vaz, B. G. Wu, and S. Zumer, "Field controlled lightscattering from nematic microdroplets," Applied Physics Letters 48 (4), 269-271 (1986).

$21 \mathrm{H}$. Ren and $\mathrm{S}$. T. $\mathrm{Wu}$, "Inhomogeneous nanoscale polymer-dispersed liquid crystals with gradient refractive index," Applied Physics Letters 81 (19), 35373539 (2002).

22 P. F. McManamon, T. A. Dorschner, D. L. Corkum, L. J. Friedman, D. S. Hobbs, M. Holz, S. Liberman, H. Q. Nguyen, D. P. Resler, R. C. Sharp, and E. A. Watson, "Optical phased array technology," Proceedings of the IEEE 84 (2), 268-298 (1996).

23 M. J. Escuti, C. C. Bowley, G. P. Crawford, and S. Zumer, "Enhanced dynamic response of the in-plane switching liquid crystal display mode through polymer stabilization," Applied Physics Letters 75 (21), 3264-3266 (1999).

24 M. Hasegawa, "Response time improvement of the in-plane-switching mode," SID Symposium Digest 28, 699 (1997).

25 R. A. M. Hikmet, "Electrically induced light-scattering from anisotropic gels," Journal of Applied Physics 68 (9), 4406-4412 (1990).

26 R. A. M. Hikmet, "Electrically induced light-scattering from anisotropic gels with negative dielectric anisotropy," Molecular Crystals and Liquid Crystals 213, 117\& (1992). 
27 F. Du and S. T. Wu, "Curing temperature effects on liquid crystal gels," Applied Physics Letters 83 (7), 1310-1312 (2003).

28 H. Ren and S. T. Wu, "Anisotropic liquid crystal gels for switchable polarizers and displays," Applied Physics Letters 81 (8), 1432-1434 (2002).

29 G. P. Crawford and S. Zumer, Liquid crystal in complex geometries. (Taylor \& Francis, London, 1996).

30 Y. K. Fung, D. K. Yang, S. Ying, L. C. Chien, S. Zumer, and J. W. Doane, "Polymer networks formed in liquid-crystals," Liquid Crystals 19 (6), 797-801 (1995).

31 R.-Q. Ma and D.-K. Yang, "Fréedericksz transition in polymer-stabilized nematic liquid crystals," Physical Review E 61 (2), 1567-1573 (2000).

32 R. A. M. Hikmet and H. M. J. Boots, "Domain-structure and switching behavior of anisotropic gels," Physical Review E 51 (6), 5824-5831 (1995).

33 V. Vorflusev and S. Kumar, "Phase-separated composite films for liquid crystal displays," SCIENCE 283 (5409), 1903-1905 (1999).

34 S. W. Kang, S. Sprunt, and L. C. Chien, "Structure and morphology of polymerstabilized cholesteric diffraction gratings," Applied Physics Letters 76 (24), 35163518 (2000).

35 W. M. Gibbons and S. T. Sun, "Optically generated liquid-crystal gratings," Applied Physics Letters 65 (20), 2542-2544 (1994).

36 T. K. Gaylord and M. G. Moharam, "Planar dielectric grating diffraction theories," Applied Physics B-Photophysics And Laser Chemistry 28 (1), 1-14 (1982). 
37 C. M. Titus, J. R. Kelly, E. C. Gartland, S. V. Shiyanovskii, J. A. Anderson, and P. J. Bos, "Asymmetric transmissive behavior of liquid-crystal diffraction gratings," Optics Letters 26 (15), 1188-1190 (2001).

38 Y. Igasaki, F. H. Li, N. Yoshida, H. Toyoda, T. Inoue, N. Mukohzaka, Y. Kobayashi, and T. Hara, "High efficiency electrically-addressable phase-only spatial light modulator," Optical Review 6 (4), 339-344 (1999).

39 W. Klaus, M. Ide, S. Morokawa, M. Tsuchiya, and T. Kamiya, "Angleindependent beam steering using a liquid crystal grating with multi-resistive electrodes," Optics Communications 138 (1-3), 151-157 (1997).

40 W. W. Chan and S. T. Kowel, "Imaging performance of the liquid-crystaladaptive lens with conductive ladder meshing," Applied Optics 36 (34), 89588969 (1997).

41 S. T. Kowel, P. Kornreich, and A. Nouhi, "Adaptive spherical lens," Applied Optics 23 (16), 2774-2777 (1984).

42 S. Masuda, S. Takahashi, T. Nose, S. Sato, and H. Ito, "Liquid-crystal microlens with a beam-steering function," Applied Optics 36 (20), 4772-4778 (1997).

43 H. Ren, L. Zhang, L. Xuan, M. Chen, and X. Huang, "A liquid crystal fresnel zone device and its light focusing properties," SID Symposium Digest 32 (1), 366-369 (2001).

44 G. Williams, N. J. Powell, A. Purvis, and M. G. Clark, "Electrically controllable liquid crystal fresnel lens," Proceedings of the SPIE 1168, 352-357 (1989).

45 N. Kitaura, S. Ogata, and Y. Mpri, "Spectrometer employing a micro-fresnel lens," Optical Engineering 34 (2), 584-588 (1995). 
46 H. H. Barrett and F. A. Horrigan, "Fresnel zone plate imaging of gamma-rays theory," Applied Optics 12 (11), 2686-2702 (1973).

47 J. Jahns and S. J. Walker, "2-dimensional array of diffractive microlenses fabricated by thin-film deposition," Applied Optics 29 (7), 931-936 (1990).

48 J. A. Davis, D. M. Cottrell, R. A. Lilly, and S. W. Connely, "Multiplexed phaseencoded lenses written on spatial light modulators," Optics Letters 14 (9), 420422 (1989).

49 M. A. A. Neil, E. G. S. Paige, and L. O. D. Sucharov, "Spatial-light-modulatorbased three-dimensional multiplanar display," Proceedings of the SPIE 3012, 337341 (1997).

50 R. Menon, E. Moon, M. Mondol, F. Castano, and H. Smith, "Scanning-spatialphase alignment for zone-plate-array lithography," JOURNAL OF VACUUM SCIENCE \& TECHNOLOGY B 22 (6), 3382-3385 (2004).

51 M. Haruna, M. Takahashi, K. Wakahayashi, and H. Nishihara, "Laser-beam lithographed micro-fresnel lenses," Applied Optics 29 (34), 5120-5126 (1990).

52 M. Li, J. Wang, L. Zhuang, and S. Y. Chou, "Fabrication of circular optical structures with a $20 \mathrm{~nm}$ minimum feature size using nanoimprint lithography," Applied Physics Letters 76 (6), 673-675 (2000).

53 J. Canning, K. Sommer, S. Huntington, and A. Carter, "Silica-based fibre fresnel lens," Optics Communications 199 (5-6), 375-381 (2001).

54 K. Rastani, A. Marrakchi, S. F. Habiby, W. M. Hubbard, H. Gilchrist, and R. E. Nahory, "Binary phase fresnel lenses for generation of 2-dimensional beam arrays," Applied Optics 30 (11), 1347-1354 (1991). 
55 E. Bricchi, J. D. Mills, P. G. Kazansky, B. G. Klappauf, and J. J. Baumberg, "Birefringent fresnel zone plates in silica fabricated by femtosecond laser machining," Optics Letters 27 (24), 2200-2202 (2002).

56 J. JAHNS and S. WALKER, "2-dimensional array of diffractive microlenses fabricated by thin-film deposition," APPLIED OPTICS 29 (7), 931-936 (1990).

57 E. G. S. Paige and L. O. D. Sucharov, "Enhancement of imaging performance of a variable focus fresnel zone plate based on a single, binary, phase-only slm," Optics Communications 193 (1-6), 27-38 (2001).

$58 \quad$ Y. Zhang and C. Zheng, "Axial intensity distribution behind a fresnel zone plate," Optics \& Laser Technology 37, 77-80 (2004).

59 T. Sakano, K. Noguchi, and T. Matsumoto, "Optical limits for spatial interconnection networks using 2-d optical array devices," Applied Optics 29 (8), 1094-1100 (1990).

60 S. Matsumoto, M. Houlbert, T. Hayashi, and K. Kubodera, "Fine droplets of liquid crystals in a transparent polymer and their response to an electric field," Applied Physics Letters 69 (8), 1044-1046 (1996).

61 V. P. Tondiglia, L. V. Natarajan, R. L. Sutherland, T. J. Bunning, and W. W. Adams, "Volume holographic image storage and electrooptical readout in a polymer-dispersed liquid-crystal film," Optics Letters 20 (11), 1325-1327 (1995).

62 H. Dammann, "Blazed synthetic phase-only holograms," Optik 31 (1), 95-\& (1970).

63 H. Ren, Y. H. Fan, and S. T. Wu, "Prism grating using polymer stabilized nematic liquid crystal," Applied Physics Letters 82 (19), 3168-3170 (2003). 
64 C. Bricot, M. Hareng, and E. Spitz, U. S. Patent No. 4,037,929 (1977).

65 H. S. Ji, J. H. Kim, and S. Kumar, "Electrically controllable microlens array fabricated by anisotropic phase separation from liquid-crystal and polymer composite materials," Optics Letters 28 (13), 1147-1149 (2003).

66 B. Wang, M. Ye, M. Honma, T. Nose, and S. Sato, "Liquid crystal lens with spherical electrode," Japanese Journal of Applied Physics 41 (11A), L1232-L1233 (2002).

67 T. Nose and S. Sato, "A liquid-crystal microlens obtained with a non-uniform electric-field," Liquid Crystals 5 (5), 1425-1433 (1989).

68 M. Ye and S. Sato, "Optical properties of liquid crystal lens of any size," Japanese Journal of Applied Physics 41 (5B), L571-L573 (2002).

69 H. Ren, Y. H. Fan, and S. T. Wu, "Tunable fresnel lens using nanoscale polymerdispersed liquid crystals," Applied Physics Letters 83 (8), 1515-1517 (2003).

70 H. Ren and S. T. Wu, "Tunable electronic lens using a gradient polymer network liquid crystal," Applied Physics Letters 82 (1), 22-24 (2003).

71 S. Gauza, H. Y. Wang, C. H. Wen, S. T. Wu, A. J. Seed, and R. Dabrowski, "High birefringence isothiocyanato tolane liquid crystals," Japanese Journal of Applied Physics 42 (6A), 3463-3466 (2003).

72 J. E. Anderson, C. Titus, P. Watson, and P. J. Bos, "Significant speed and stability increases in multi-dimensional director simulations," SID Symposium Digest 31 (1), 906-909 (2000).

73 M. V. K. Chari and S. J. Salon, Numerical methods in electromagnetism. (Academic Press, San Diego, 2000), pp.xiv, 767. 
74 K. R. Welford and J. R. Sambles, "Analysis of electric-field induced deformations in a nematic liquid-crystal for any applied field," Molecular Crystals and Liquid Crystals 147, 25-42 (1987).

75 M. Born and E. Wolf, Principles of optics: Electromagnetic theory of propagation, interference and diffraction of light, 6th (corr.) ed. (Cambridge University Press, Cambridge, UK; New York, 1997), pp.xxviii, 808.

76 Y. Aoki, Y. Shimada, and K. Iga, "Evaluation of numerical aperture and focusing characteristics of planar microlens for optical interconnects," Japanese Journal of Applied Physics Part 2-Letters 40 (5A), L446-L448 (2001).

77 J. Duparre, B. Gotz, and R. Goring, "Micro-optical 1 x 4 fiber switch for multimode fibers with 600-mu m core diameters," APPLIED OPTICS 42 (34), 6889-6896 (2003).

78 J. Duparre, D. Radtke, and P. Dannberg, "Implementation of field lens arrays in beam-deflecting microlens array telescopes," Applied Optics 43 (25), 4854-4861 (2004).

79 Y. Fu and N. K. A. Bryan, "Design of hybrid micro-diffractive-refractive optical element with wide field of view for free space optical interconnections," Optics Express 10 (13), 540-549 (2002).

80 P. Smith, C. Taylor, E. McCabe, D. Selviah, S. Day, and L. Commander, "Switchable fiber coupling using variable-focal-length microlenses," Review of Scientific Instruments 72 (7), 3132-3134 (2001). 
81 B. Lee, S. Jung, S.-W. Min, and J.-H. Park, "Three-dimensional display by use of integral photography with dynamically variable image planes," Optics Letters 26 (19), 1481-1482 (2001).

82 Y. Choi, J. H. Park, J. H. Kim, and S. D. Lee, "Fabrication of a focal length variable microlens array based on a nematic liquid crystal," Optical Materials 21 (1-3), 643-646 (2003).

83 L. G. Commander, S. E. Day, and D. R. Selviah, "Variable focal length microlenses," Optics Communications 177 (1-6), 157-170 (2000).

84 H. Ren, Y. H. Fan, and S. T. Wu, "Liquid-crystal microlens arrays using patterned polymer networks," Optics Letters 29 (14), 1608-1610 (2004).

85 H. Ren, Y. H. Fan, S. Gauza, and S. T. Wu, "Tunable microlens arrays using polymer network liquid crystal," Optics Communications 230 (4-6), 267-271 (2004).

86 H. K. Bucher, R. T. Klingbiel, and J. P. VanMeter, "Frequency-addressed liquidcrystal field-effect," Applied Physics Letters 25 (4), 186-188 (1974).

87 M. Schadt, "Low-frequency dielectric relaxations in nematics and dual-frequency addressing of field effects," Molecular Crystals and Liquid Crystals 89 (1-4), 7792 (1982).

88 I. C. Khoo and S. T. Wu, Optics and nonlinear optics of liquid crystals. (World Scientific, Singapore, New Jersey, London, Hong Kong, 1993).

89 S. T. Wu, in Optical materials, edited by S. Musikant (Marcel Dekker, New York, 1990). 
90 H. Ren, Y. H. Fan, S. Gauza, and S. T. Wu, "Tunable-focus flat liquid crystal spherical lens," Applied Physics Letters 84 (23), 4789-4791 (2004).

91 T. Okamoto, M. Mori, T. Karasawa, S. Hayakawa, I. Seo, and H. Sato, "Ultraviolet-cured polymer microlens arrays," Applied Optics 38 (14), 2991-2996 (1999).

92 S. T. Wu, "Nematic liquid-crystal modulator with response-time less than 100mu-s at room-temperature," Applied Physics Letters 57 (10), 986-988 (1990).

93 R. A. M. Hikmet, "Anisotropic gels and plasticized networks formed by liquidcrystal molecules," Liquid Crystals 9 (3), 405-416 (1991).

94 S.-T. Wu and D.-K. Yang, Reflective liquid crystal displays. (Wiley, Chichester Eng.; New York, 2001), pp.xvi, 335.

95 Y. H. Fan, Y. H. Lin, H. Ren, S. Gauza, and S. T. Wu, "Fast-response and scattering-free polymer network liquid crystals for infrared light modulators," Applied Physics Letters 84 (8), 1233-1235 (2004).

96 D.-K. Yang, L. C. Chien, and Y. K. Fung, in Liquid crystals in complex geometries formed by polymer and porous networks, edited by G. P. Crawford and S. Zumer (Taylor \& Francis, London, 1996).

97 Y. H. Fan, H. Ren, and S. T. Wu, "Normal-mode anisotropic liquid-crystal gels," Applied Physics Letters 82 (18), 2945-2947 (2003).

98 Y. Fung, A. Borstnik, S. Zumer, D. Yang, and J. Doane, "Pretransitional nematic ordering in liquid crystals with dispersed polymer networks," PHYSICAL REVIEW E 55 (2), 1637-1645 (1997). 
99 T. J. Bunning, L. V. Natarajan, V. P. Tondiglia, and R. L. Sutherland, "Holographic polymer-dispersed liquid crystals (h-pdlcs)," Annual Review of Materials Science 30, 83-115 (2000).

100 J. L. West, G. Zhang, and A. Glushchenko, "Stressed liquid crystals for electrically controlled fast shift of phase retardation," SID Symposium Digest 34 (1), 1469-1471 (2003).

101 O. A. APHONIN, Y. V. PANINA, A. B. PRAVDIN, and D. A. YAKOVLEV, "Optical-properties of stretched polymer-dispersed liquid-crystal films," LIQUID CRYSTALS 15 (3), 395-407 (1993).

102 B. G. Wu, J. H. ERDMANN, and J. W. DOANE, "Response-times and voltages for pdlc light shutters," LIQUID CRYSTALS 5 (5), 1453-1465 (1989).

103 S. T. Wu and C. S. Wu, "Experimental confirmation of the osipov-terentjev theory on the viscosity of nematic liquid-crystals," Physical Review A 42 (4), 2219-2227 (1990).

104 I. Haller, "Thermodynamic and static properties of liquid crystals," Progress in Solid State Chemistry 10, 103-112 (1975).

105 S. T. Wu, "Birefringence dispersions of liquid-crystals," Physical Review A 33 (2), 1270-1274 (1986). 